DOE/EV/00889-7

VOL. 2

PROGRESS REPORT

CONTRACT DE-AS09-76EV00889

Larry P. Atkinson

-SKIO-

VOLUME 2

GABEX-1 


\section{DISCLAIMER}

This report was prepared as an account of work sponsored by an agency of the United States Government. Neither the United States Government nor any agency Thereof, nor any of their employees, makes any warranty, express or implied, or assumes any legal liability or responsibility for the accuracy, completeness, or usefulness of any information, apparatus, product, or process disclosed, or represents that its use would not infringe privately owned rights. Reference herein to any specific commercial product, process, or service by trade name, trademark, manufacturer, or otherwise does not necessarily constitute or imply its endorsement, recommendation, or favoring by the United States Government or any agency thereof. The views and opinions of authors expressed herein do not necessarily state or reflect those of the United States Government or any agency thereof. 


\section{DISCLAIMER}

Portions of this document may be illegible in electronic image products. Images are produced from the best available original document. 


\title{
FIRST PRELIMINARY REPORT OF THE GABEX-1 CRUISES: \\ STATION LOGS, STANDARD SECTIONS AND MAPS AND \\ TIME SERIES TRANSECTS.
}

\author{
By \\ Larry P. Atkinson \\ James J. Singer \\ William S. Chandler \\ July 8,1980
}


This preliminary cruise report contains information that all GABEX participants may find useful, especially the organization of sections and maps. We have tried to look at the organization of the cruises in a variety of ways all of which are presented in the following. Examples of standard plots are also included along with information on how to specify plots you may need.

Temperature and salinity printouts are not included to save paper. They are available to anyone but it would be easier if you requested specific stations, sections or maps. The nutrient, chlorophyll and dissolved oxygen data are not yet entered into the data set so if you need those data make specific requests. We expect all data to be fully merged by mid-August when the full station lists can be made.

Station log listings for the EASTWARD and ISELIN are included. (Appendices 1 and 2.) 
The first two figures (Figures 1 and 2) show the cruise tracks for each. ship. This is more for the humor than anything. It clearly show that the ISELIN was following events while the EASTWARD was running a set grid.

The data are broken up into maps and sections which consist of data from one ship usually but not always,

The ISELIN ran 24 sections (Figure 3 and Table 1). Most sections were trans-shelf although some were run along-shore at the $60 \mathrm{~m}$ isobath. When you refer to a section include the ship name, re. ISELIN Section. 4. The table gives the station numbers that make up a section or map.

The $60 \mathrm{~m}$ isobath runs (Figure 4 and Table 2) consists of three ISELIN sections and one EASTWARD section. Initial temperature plots indicate that the individual sections cannot be combined. This is because shelfbreak events propogate north ca. .8 knot.

The EASTWARD was running a standard grid of stations so grid numbers were established. Thus most EASTWARD stations are identified by both consecutive number as well as grid number. Figure 5 shows the standard grid.

The EASTWARD ran 20 sections (Figure 6 and Table 3 ) one of which was partly alongshore on the $60 \mathrm{~m}$ isobath.

The St. Augustine section $\left(30.0^{\circ} \mathrm{N}\right)$ was occupied 11 times (including one EASTWARD section that was. 5 n.m. south). The standard section is stations 13-25 in Figure 5. Table 4 contains relevant data.

Maps were made at the shelfbreak in our effort to detect upiwelling events. The resulting data from 9 maps applicable mostly to surface data. Maps 1-7 contain only ISELIN data while 8 and 9 also include EASTWARD data. Figures 7-15 and Table 5 show the maps. 
The EASTWARD data were arranged into three maps, two of which contain ISELIN data (Figures 16-18, Táble 6).

Another way to organize the data are by time-latitude plots. Figure 19 shows the EASTWARD stations plotted in this way with section numbers included. ISELIN cross sheif sections are indicated in Figure 20 and the $60 \mathrm{~m}$ isobath runs in Figure 21. The composite maps are shown in Figure 22. NASA Flights

The $U-2$ and $C-130$ made flights on 15 April. Figure 23 shows the flight track and Figure 24 shows the latitude/time plot for the flight and coincident ISELIN stations. Tracks and turn points are given in Tables 7 and 8. Coast Guard Flights

To follow.

Standardized Plotting

To assure uniform and comparible plots I suggest that you use, if possible, the standard products we can supply. Cross-Shelf Transects

We are plotting these on a standard $200 \mathrm{~m}$ deep by $160 \mathrm{~km}$ section. The two reduced examples show a standard plot with sampling points marked. The latter may be particularly useful for much of the shipboard subsurface sampling. We can supply either type plotted by standard pen on bond (for contouring, etc.) or Leroy on tracing paper for photo quality. Specify the section you want plotted by section number and ship. Contact Bill Chandler for these plots (912-356-2459).

Horizontals

Horizontal plots will be done on at least two scales: $28-32^{\circ} \mathrm{N}, 79-82^{\circ} \mathrm{W}$ and $28.5-32^{\circ} \mathrm{N}, 79.5-80.5^{\circ} \mathrm{W}$. Samples are enclosed. 


\section{TABLES}

\section{ISELIN Sections}

2. $60 \mathrm{~m}$ Isobath Runs

3. EASTWARD Sections

4. St. Augustine Time Series Transect $\left(30.0^{\circ} \mathrm{N}\right)$

5. Composite ISELIN Maps

6. EASTWARD Maps

7. C-130 Flight Path

8. U-2 Flight Path 
Table 1. ISELIN SECTIONS

Section . Location

1. Brunswick

2 St. Augustine

3

4

5

6

7

8

9

10

11

12

13

14

15

16

17

18

19

20

21

22

23

24
Stations (Grid)

19-27

$29-33(13-18)$

$34-40(13-18)$

41-47 (13-18)

72-89

139-144

145-151 (13-19)

153-159. (13-19)

181-186

187-191

195-198

204-207

209-213

217-221

223-225

22.8-234

242-261

$262-2.67(13-18)$

280-294

295-299

301-306

$32 b-329$

$336-340$

344-351
Time Interval

$0900 / 11 / 4-0100 / 12 / 4$

$0800 / 12 / 4-1746 / 12 / 4$

$1850 / 12 / 4-2100 / 12 / 4$

$0715 / 13 / 4-1642 / 13 / 4$

$2040 / 14 / 4$

$1915 / 16 / 4-2134 / 16 / 4$

$0837 / 17 / 4-1942 / 17 / 4$

$0708 / 18 / 4-1636 / 18 / 4$

$1042 / 19 / 4-1155 / 19 / 4$

$1344 / 19 / 4-1507 / 19 / 4$

$1910 / 19 / 4-2315 / 19 / 4$

$0546 / 20 / 4-0948 / 19 / 4$

$1310 / 20 / 4-0138 / 21 / 4$

$0614 / 21 / 4-1040 / 21 / 4$

$1324 / 21 / 4-1532 / 21 / 4$

$2010 / 21 / 4-0235 / 22 / 4$

$0912 / 22 / 4-1855 / 22 / 4$

$2010 / 22 / 4-0400 / 23 / 4$

$2025 / 23 / 4-0345 / 24 / 4$

$0459 / 24 / 4-1221 / 24 / 4$

$1530 / 24 / 4-1731 / 24 / 4$

$1153 / 25 / 4-1757 / 25 / 4$

$0028 / 26 / 4-0756 / 26 / 4$

$1235 / 26 / 4-2151 / 26 / 4$ 
Table 2. $60 \mathrm{M}$ ISOBATH TRANSECTS

During the cruise we made several runs along the $60 \mathrm{~m}$ isobath. The main run was section 17. EZ section 20 was a 150 a short $60 \mathrm{~m}$ run.

Ship

CI

CI

CI

$E Z$
Section

17

19

21

20
Time Interval

0912 - $1855 / 22$

$2025 / 23-0345 / 24 / 4$

$1530-1731 / 24 / 4$

$2119-2220 / 26$ 
Table 3. EASTWARD SECTIONS

\begin{tabular}{|c|c|c|c|}
\hline Section & Location & Stations (Grid) & Time Interval \\
\hline 1 & New Smyrna & $31-41(1-11)$ & $0357 / 12 / 4-1330 / 12 / 4$ \\
\hline 2 & St. Augustine & $48-56(17-25)$ & $1811 / 12 / 4-0205 / 13 / 4$ \\
\hline 3 & Brunswick & $63-80(26-43)$ & $0830 / 13 / 4-0100 / 14 / 4$ \\
\hline 4 & Savannah & $88-92(44-48)$ & $0449 / 14 / 4-1305 / 14 / 4$ \\
\hline 5 & St: Augustine & $111-124(13-25)$ & $0804 / 15 / 4-2318 / 15 / 4$ \\
\hline 6 & New Smyrna & $132-142(1-11)$ & $0912 / 16 / 4-1728 / 16 / 4$ \\
\hline 7. & St. Augustine & $149-161(13-25)$ & $2211 / 16 / 4-0907 / 17 / 4$ \\
\hline 8 & Brunswick & $169-185(26-42)$ & $1610 / 17 / 4-0557 / 18 / 4$ \\
\hline 9. & Savannah & $194-212(44-62)$ & $1126 / 18 / 4-0216 / 19 / 4$ \\
\hline 10 & St. Augustine & $225-237(13-25)$ & $1435 / 19 / 4-0231 / 20 / 4$ \\
\hline 11 & New Smyrna & $245-258(1-11)$ & $1034 / 20 / 4-2330 / 20 / 4$ \\
\hline 12 & St. Augustine & $269-281(13-25)$ & $0517 / 21 / 4-1702 / 21 / 4$ \\
\hline 13 & Brunswick & $288-304(26-42)$ & $0002 / 22 / 4-1237 / 22 / 4$ \\
\hline 14 & Savannah & $313-331 \quad(44-62)$ & $1746 / 22 / 4-.0908 / 23 / 4$ \\
\hline 15 & St. Augustine & $344-356(13-25)$ & $2145 / 23 / 4-0907 / 24 / 4$ \\
\hline 16 & $\begin{array}{l}\text { "10" n.m. S: } \\
\text { st. Augustine }\end{array}$ & $364-374$ & $1436 / 24 / 4-1810 / 24 / 4$ \\
\hline 17. & New Smyrna & $380-390(1-11)$ & $2334 / 24 / 4-1036 / 25 / 4$ \\
\hline 18 & $\begin{array}{l}5 \text { n.m. S: } \\
\text { St. Augustine }\end{array}$ & $397-407$ & $1559 / 25 / 4-0335 / 26 / 4$ \\
\hline $19^{\circ}$ & $\begin{array}{l}5 \text { n.m. S. } \\
\text { Brunswick }\end{array}$ & $417-424$ & $1040 / 26 / 4-1923 / 26 / 4$ \\
\hline 20 & $\begin{array}{l}10 \text { n.m. N. } \\
\text { Brunswick }\end{array}$ & $429-432$ & $2237 / 26 / 4-0126 / 27 / 4$ \\
\hline
\end{tabular}


Table 4. ST. AUGUSTINE TIME SERIES

Both CI and EZ occupied the St. Augustine section many times. Following is a composite time series section list.

$\begin{array}{lccc}\text { Ship } & \frac{\text { Section }}{2} & \frac{\text { Grid Stations }}{13-18} & \frac{\text { Time Interval }}{0800 / 12 / 4-1746 / 12 / 4} \\ \text { CI } & 2 & 17-25 & 1811 / 12 / 4-0205 / 13 / 4 \\ \text { EZ } & 2 & 13-18 & 1850 / 12 / 4-2100 / 12 / 4 \\ \text { CI } & 3 & 13-18 & 0715 / 13 / 4-1642 / 13 / 4 \\ \text { CI } & 4 & 13-25 & 0804 / 15 / 4-2318 / 15 / 4 \\ \text { EZ } & 5 & 13-25 & 2211 / 16 / 4-0907 / 17 / 4 \\ \text { EZ } & 7 & 13-25 & 1435 / 19 / 4-0231 / 20 / 4 \\ \text { EZ } & 10 & 13-25 & 0517 / 21 / 4-1702 / 21 / 4 \\ \text { EZ } & 12 & 13-18 & 2010 / 22 / 4-0400 / 23 / 4 \\ \text { CI } & 18 & 13-25 & 2145 / 23 / 4-0907 / 24 / 4 \\ \text { EZ } & 15 & & 1559 / 25 / 4-0335 / 26 / 4 \\ \text { EZ } & 18^{*} & & \end{array}$

*Section 5 miles south of St. Augustine. 
Table 5. COMPOSITE MAPPING RUNS

3

STATIONS

MAP

ISELIN

EASTWARD

1

$1-18$

2

$49-59$

3

$86-144$

4

$160-198$

5

$195-236$

6

$228-269$

7

269-299

8

295-323

$344-356$

$364-374$

$380-390$

9

$324-351$

$401-423$

in

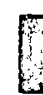

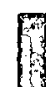




\section{Table 6. EASTWARD MAPS}

The Eastward stations can be used in various ways to create maps. The combination we have chosen is as follows:

\begin{tabular}{|c|c|c|c|}
\hline Map. & Sections & Stations & Time Interval \\
\hline \multirow[t]{2}{*}{$1^{*}$} & $1-4$ & $31-92$ & $0357 / 12 / 4-1305 / 14 / 4$ \\
\hline & CI 5 . & $72-89$ & $14-15 / 4$ \\
\hline \multirow[t]{2}{*}{$2+$} & $6-9$ & $132-212$ & $0912 / 16 / 4-0216 / 19 / 4$ \\
\hline & CI $6{ }^{\circ}$ & $139-144$ & $16 / 4$ \\
\hline 3 & $1-4$ & $245-331$ & $1034 / 20 / 4-0908 / 23 / 4$ \\
\hline
\end{tabular}

*Because of weather the EZ could not complete sections. CI ran full section on 14-15 April (Stations 72-89) which could be added to complete section.

tAdd ISELIN C. Canaveral section 6 (stations $139-144$ on $16 / 4$ ). 
Table 7. C- 130 Apri.1 15, 1980 Afternoon

\begin{tabular}{|c|c|c|c|}
\hline Sect & 1 & $30^{\circ} 55^{\prime}$ & $79^{\circ} 55^{\prime}$ \\
\hline & & $37^{\circ} 08^{\prime}$ & $79^{\circ} 37^{\prime}$ \\
\hline " & 2 & $30^{\circ} 52^{\prime}$ & $79^{\circ} 51$ \\
\hline & & $31^{\circ} 11^{i}$ & $79^{\circ} 18^{\prime}$ \\
\hline$\because$ & 3 & $30^{\circ} 55^{\prime}$ & $79^{\circ} 38^{\prime}$ \\
\hline & & $31^{0} 11^{\prime}$ & $79^{\circ} 09^{\prime}$ \\
\hline$\pi$ & 4 & $30^{\circ} 52^{\prime}$ & $79^{\circ} 35^{\prime}$ \\
\hline & & $31^{\circ} 09^{\prime}$ & $79^{\circ} 38^{\prime}$ \\
\hline.$"$ & 5 & $30^{\circ} 55^{\prime}$ & $79^{\circ} 26^{\prime}$ \\
\hline & & $3.1^{\circ} 12^{\prime}$ & $79^{\circ} 29^{\prime}$ \\
\hline$"$ & 6 & $30^{\circ} 25^{\prime}$ & $79^{\circ} 40^{\prime}$ \\
\hline & & $30^{\circ} 20^{\prime}$ & $80^{\circ} 55^{\prime}$ \\
\hline$"$ & 7 & $30^{\circ} 20^{\prime}$ & $80^{\circ} 55^{\prime}$ \\
\hline & & $30^{\circ} 20^{\prime}$ & $79^{\circ} 40^{\prime}$ \\
\hline " & 8 & $30^{\circ} 20^{\prime}$ & $79^{\circ} 40^{\prime}$ \\
\hline & & $30^{\circ} 16^{\prime}$ & $80^{\circ} 4$ \\
\hline
\end{tabular}


Table 8. U-2 OCS Ground Track - April 15, 1980 Afternoon

\begin{tabular}{|c|c|c|c|}
\hline \multirow[t]{2}{*}{$n$ Points } & 1 & $30^{\circ} 33^{\prime}$ & $80^{\circ} 53^{\prime}$ \\
\hline & 2 & $31^{\circ} 27^{i}$ & $79^{\circ} 20^{\prime}$ \\
\hline & 3 & $31^{\circ} 11^{\prime}$ & $7.9^{\circ} 10^{\prime}$ \\
\hline & 4 & $30^{\circ} \cdot 24^{\prime}$ & $80^{\circ} 40^{\prime}$ \\
\hline & 5 & $30^{\circ} 16^{\prime}$ & $80^{\circ} 30^{\prime}$ \\
\hline & 6 & $30^{\circ} 46^{\prime}$ & $79^{\circ} 20^{\prime}$ \\
\hline & 7 & $30^{\circ} 20^{\prime}$ & $79^{\circ} 20^{\prime}$ \\
\hline : & 8 & $30^{\circ} 06 !$ & $80^{\circ} 43^{\prime}$ \\
\hline & 9 & $29^{\circ} 54^{\prime}$ & $80^{\circ} 40^{\prime}$ \\
\hline & 10 & $30^{\circ} 10^{\prime}$ & $79^{\circ} 10^{\prime}$ \\
\hline & 11 & $30^{\circ} 22^{\prime}$ & $79^{\circ} 20^{\prime}$ \\
\hline & 12 & $30^{\circ} 22^{\prime}$ & $81^{\circ} 23^{\prime}$ \\
\hline
\end{tabular}




\section{FIGURE CAPTIONS}

1. EASTWARD cruise track.

2. ISELIN cruise track.

3. ISELIN sections. Section number indicated. Refer to Table 1 for stations, etc.

4. ISELIN stations on $60 \mathrm{~m}$ isobath. Refer to Table 2 for stations, etc.

5. EASTWARD standard grid stations. Stations 13-25 make up the St. Augustine time series section. Station 12 is not part of the grid.

6. EASTWARD sections. Section numbers indicated. Refer to Table 3 for stations, etc.

7. ISELIN surface map \#1. Refer to Table 6.
8.

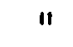
"1 . H2
9.

" .
\#3.
10.

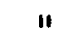
11
\#4.
11. : :
12. "
13. "
14. "
15.

" 1

16. EASTWARD Map \#1. Refer to Table 6.

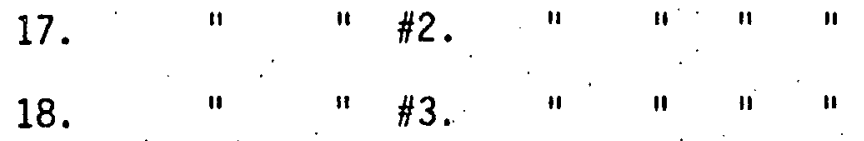

19. Time-Latitude chart of EASTWARD stations.

20. " " "ISELIN Cross Shelf Stations. Sections indicated.

21. " " "ISELIN $60 \mathrm{~m}$ isobath run. Sections indicated.

22. " " "maps. Map \# indicated. Dashed line indicated U-2 and C-130 flight.

23. U-2 and C-130 flight track. Refers to Table 7 \& 8 . 


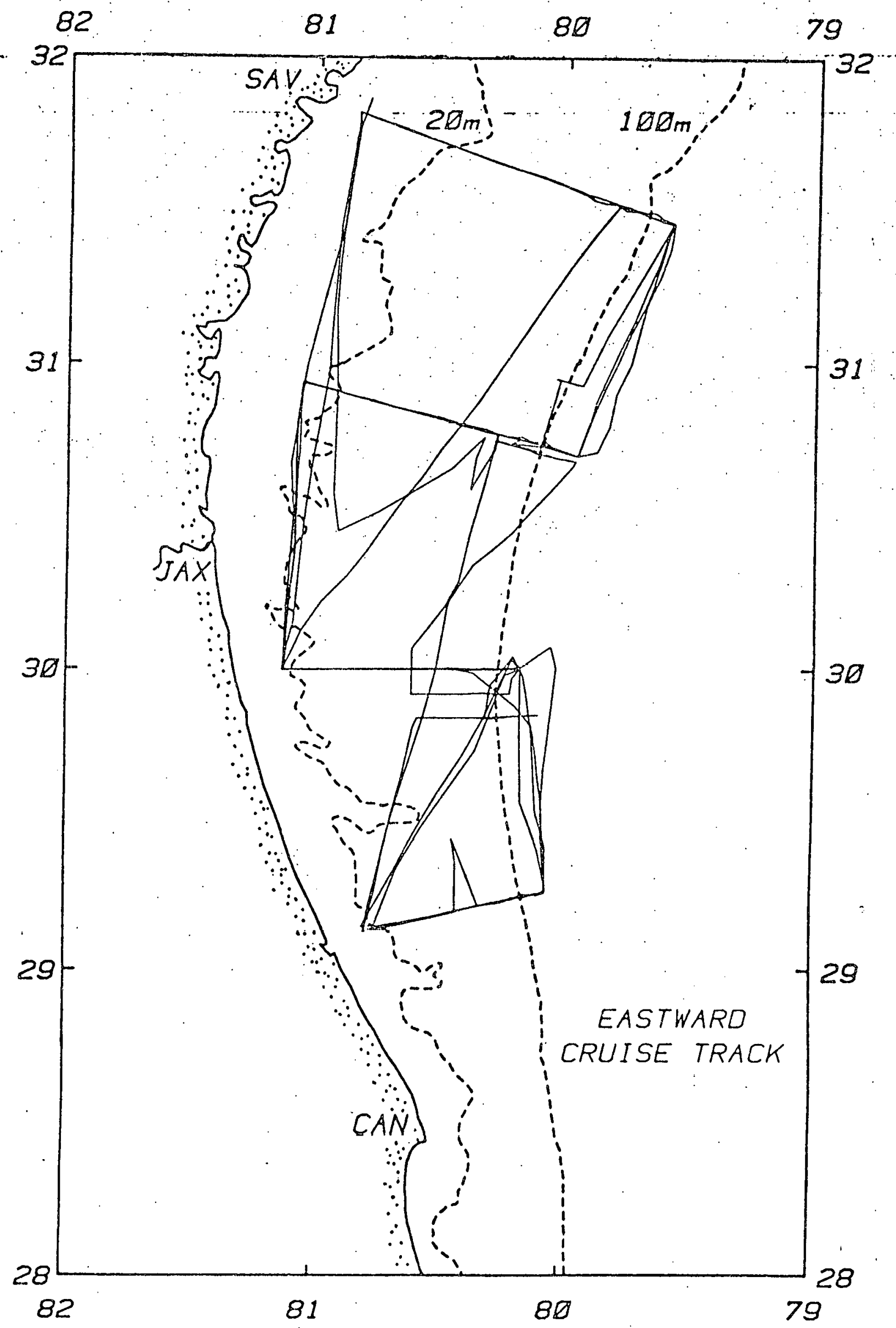

Figure 1. EASTWARD cruise track 


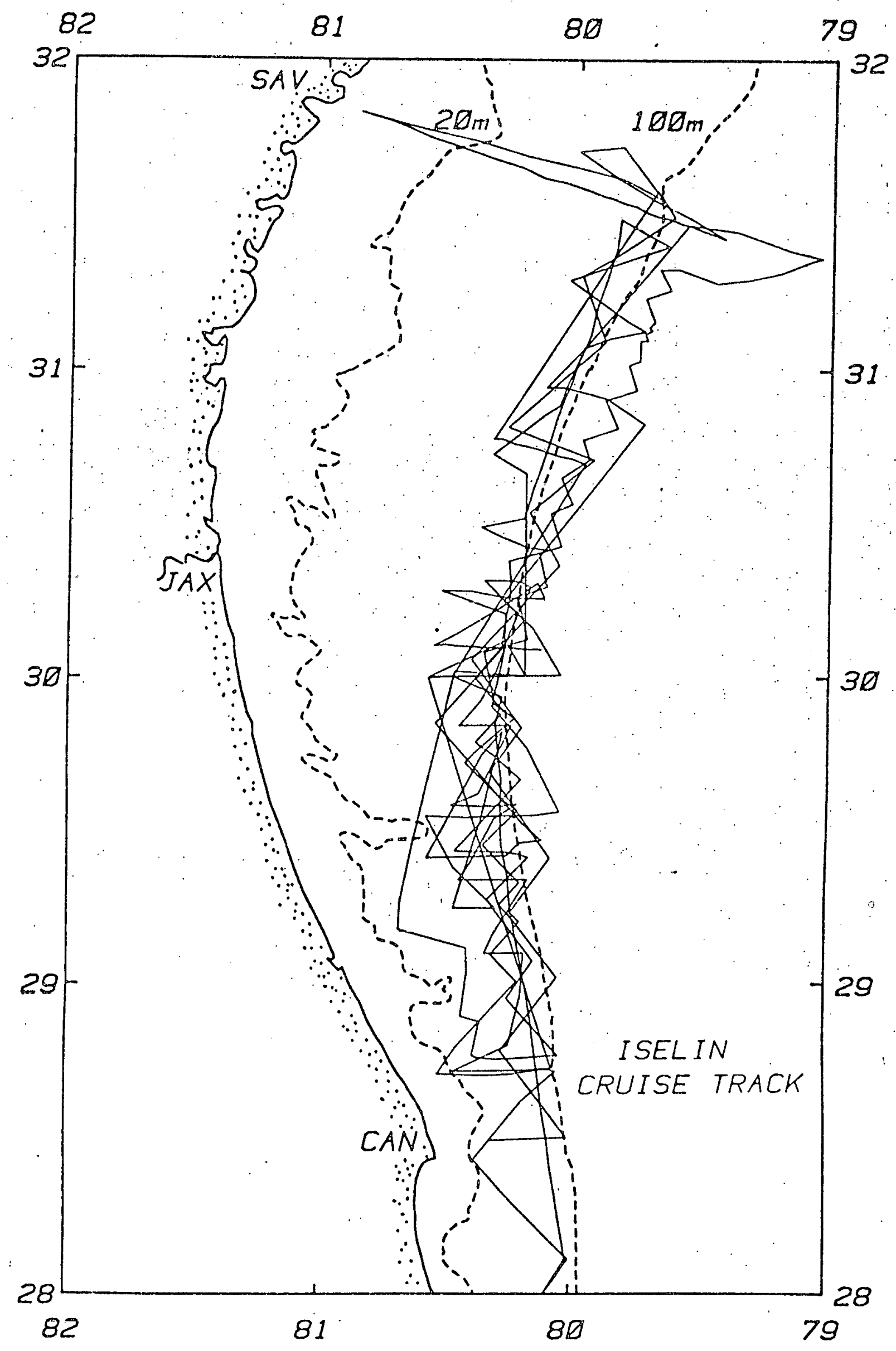

Figure 2. ISELIN cruise track 


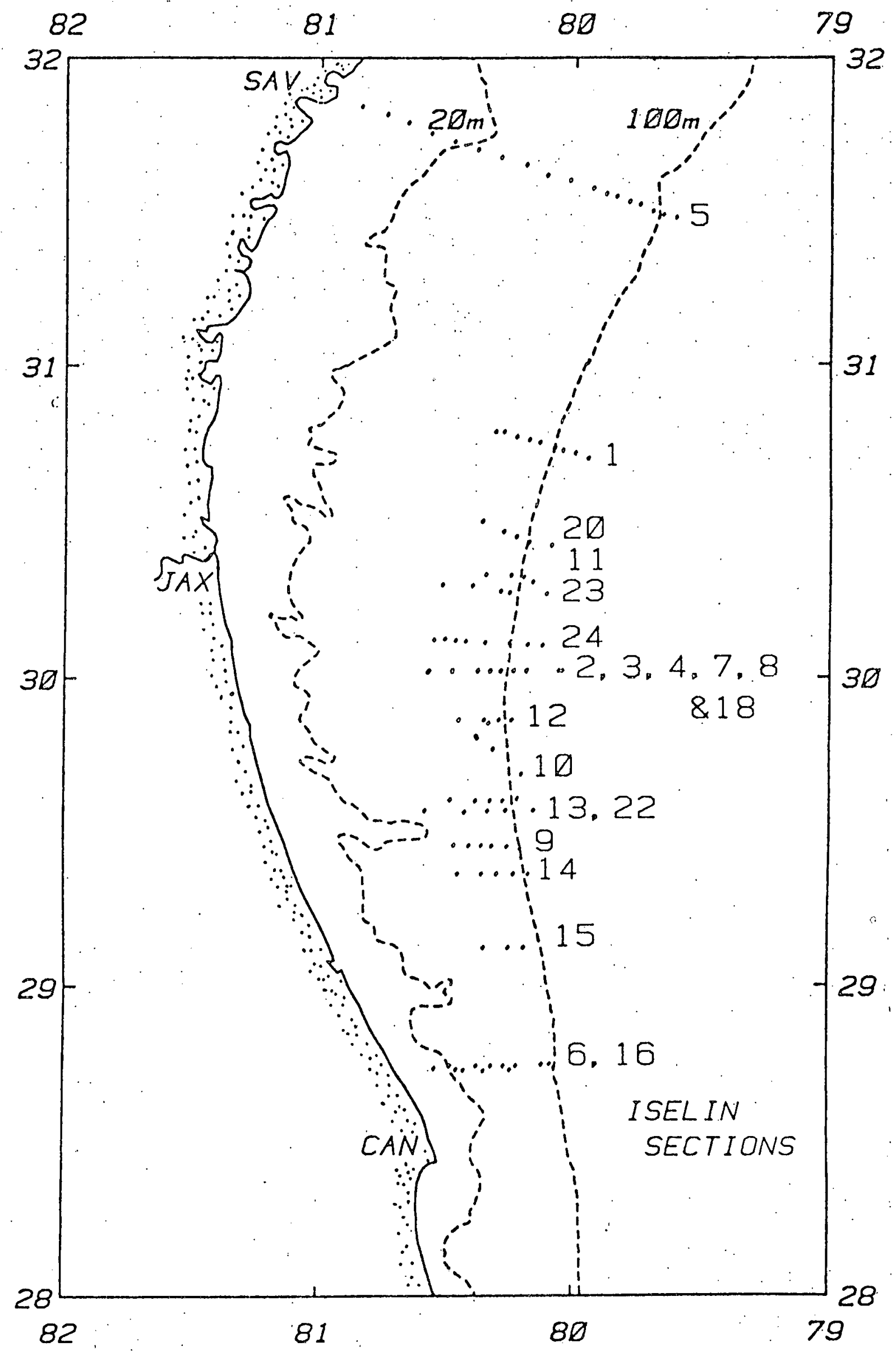

Figure 3. ISELIN sections. Section number indicated. Refer to Table 1 for stations; etc. 


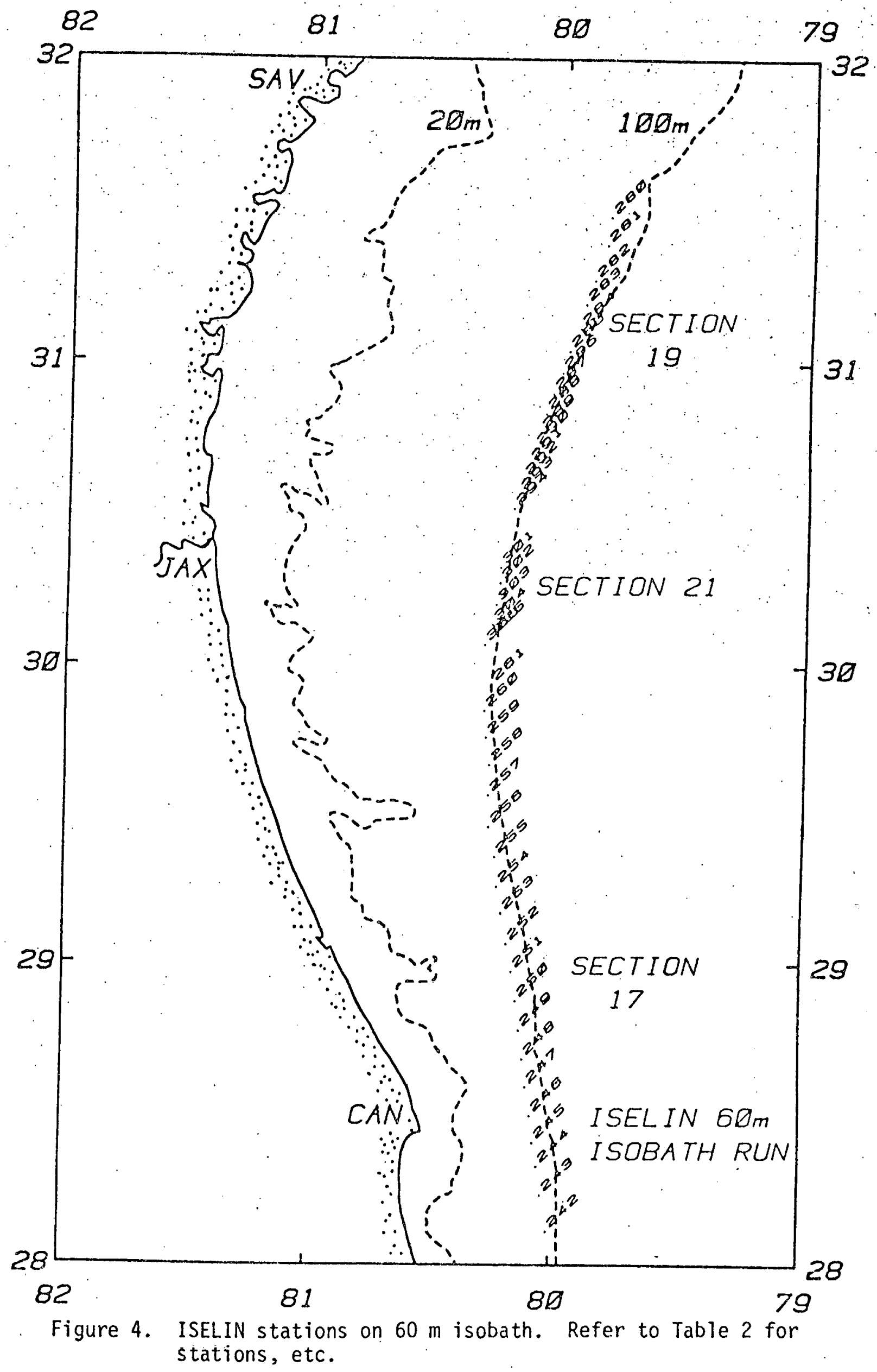




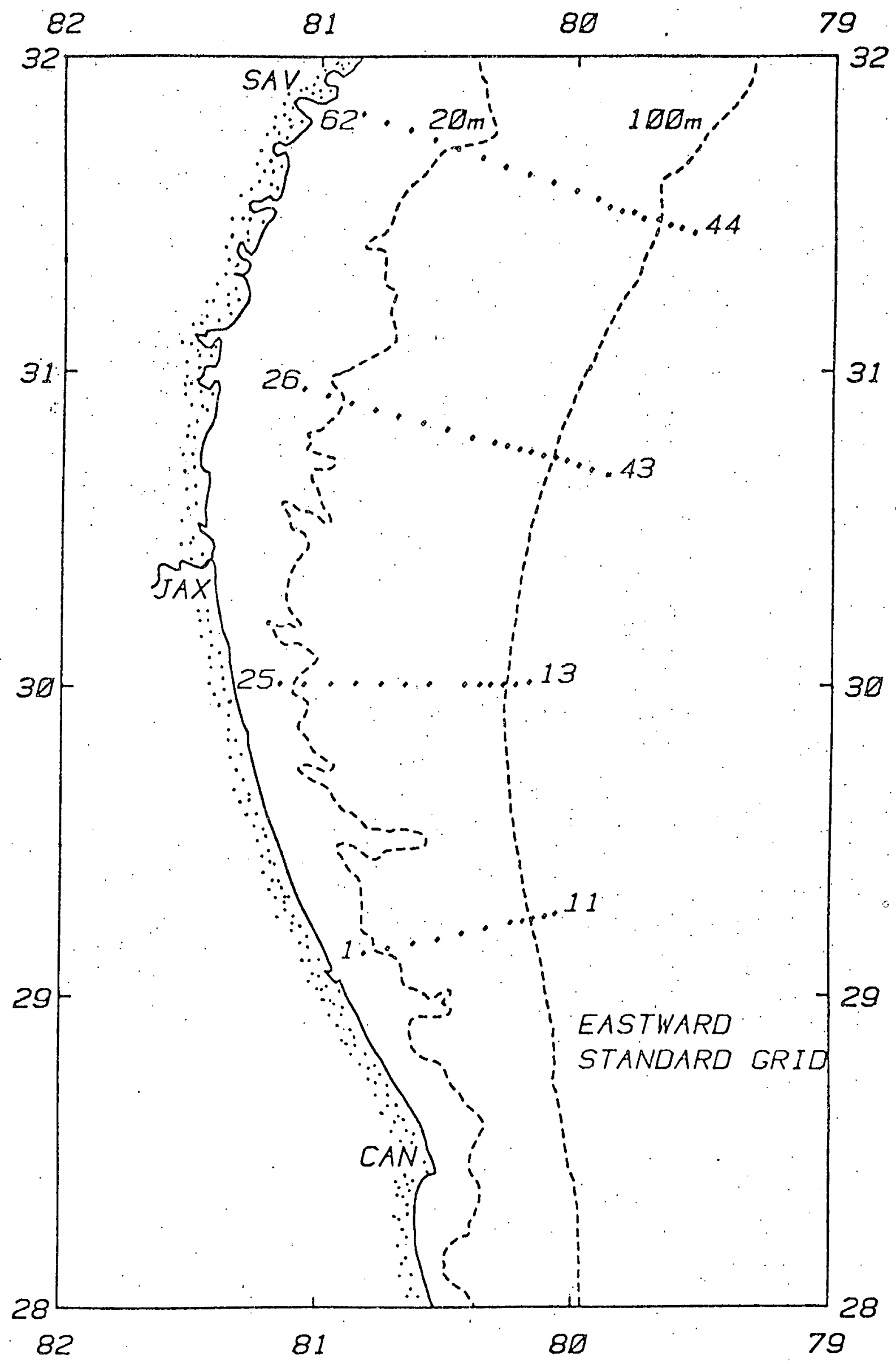

Figure 5. EASTWARD standard grid stations. Stations 13-25 make up the St. Augystine time series section. Station 12 is not part of the grid. 


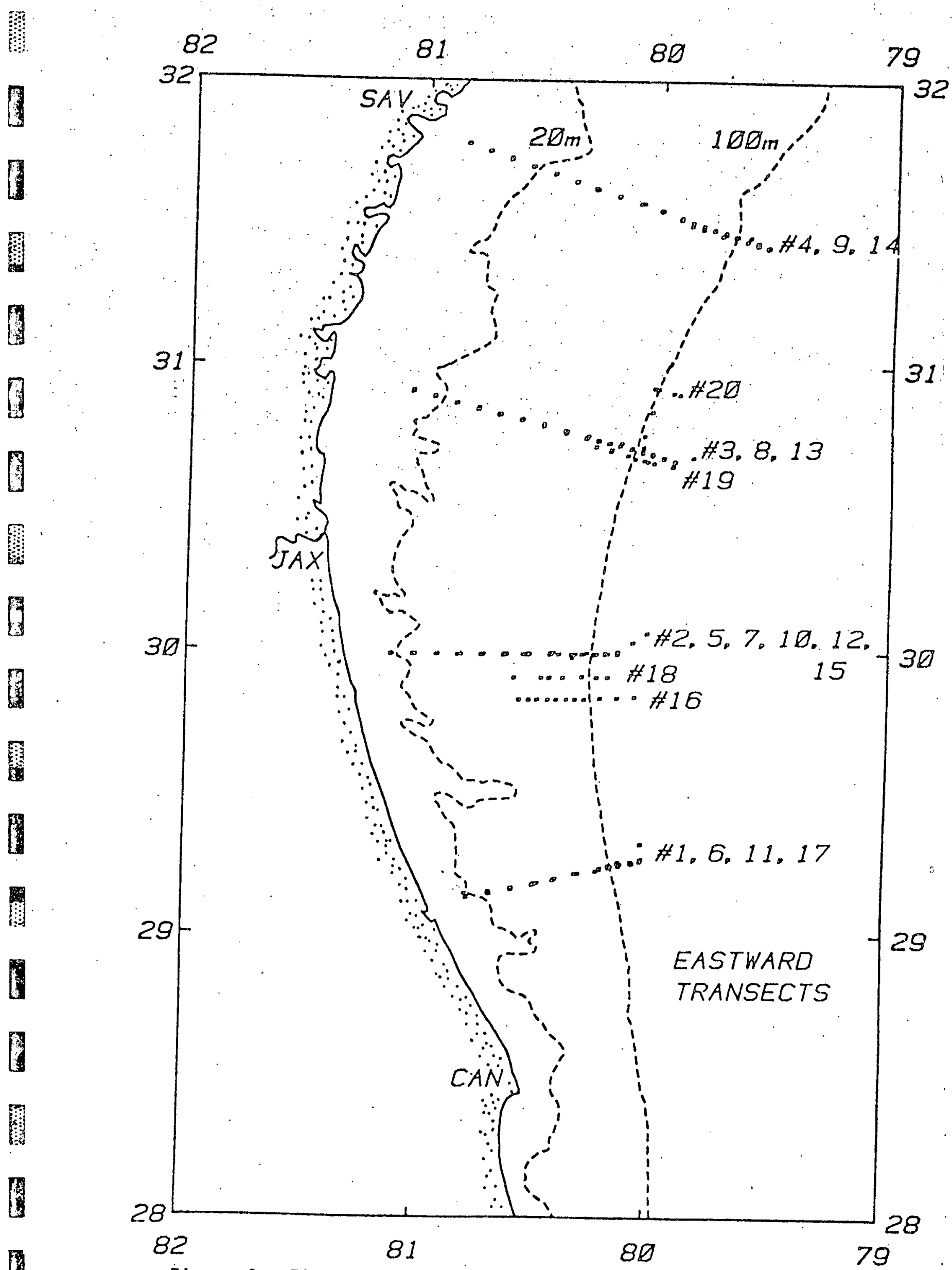

Figure 6. EASTWARD sections. Section numbers indicated. Refer to Table 3 for stations, etc. 


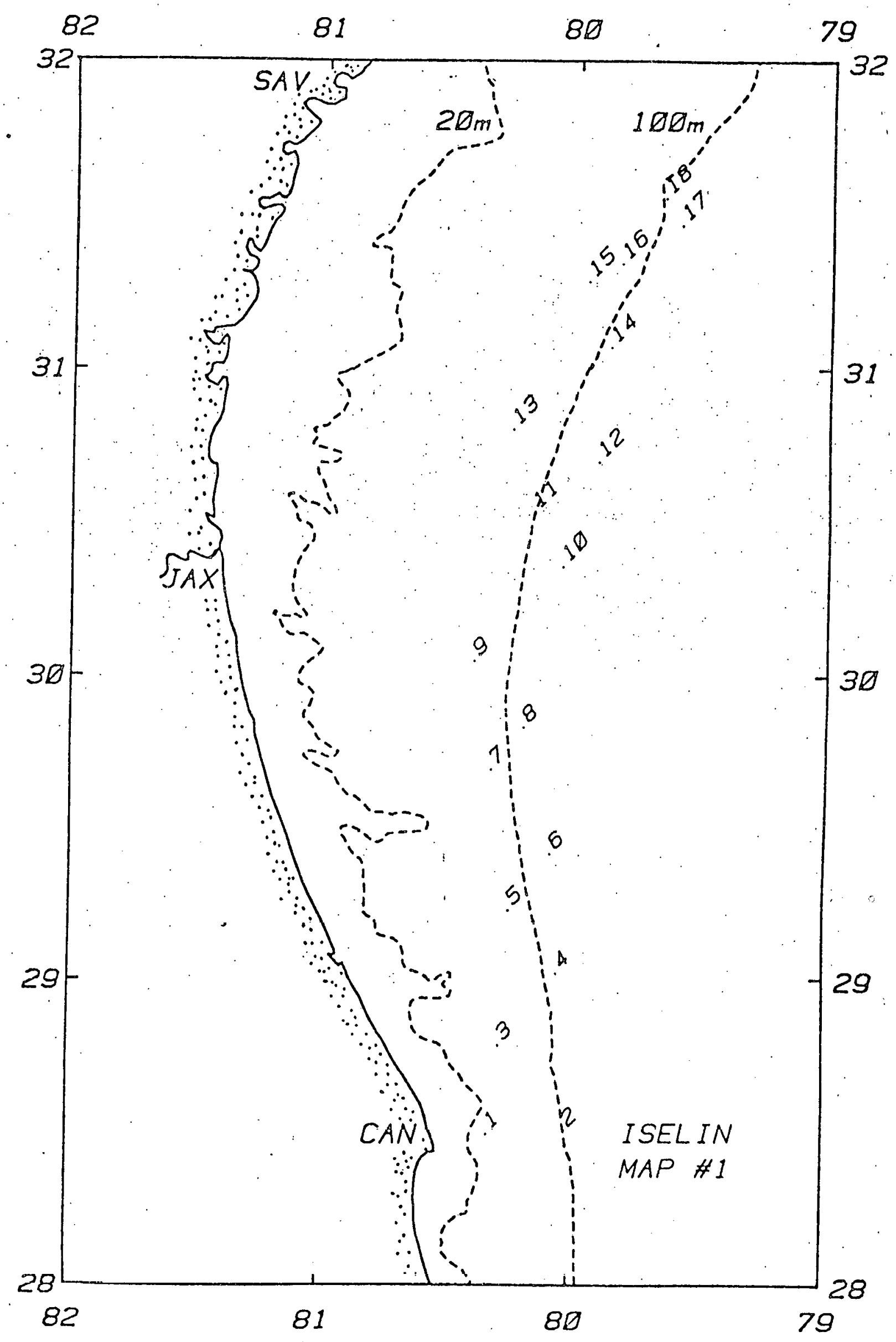

Figure 7. ISELIN surface map. \#1. Refer to Table 6 . 


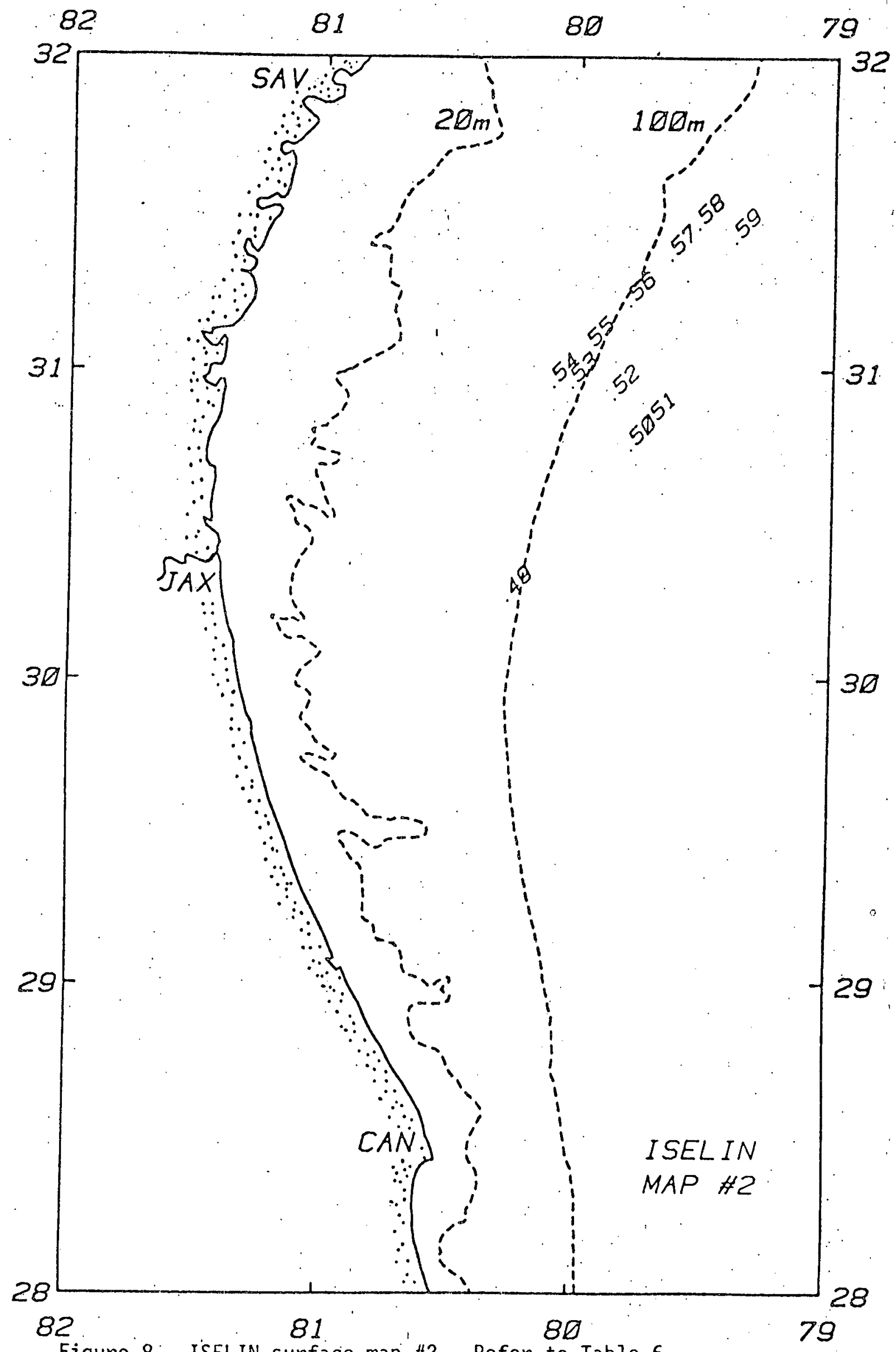

Figure 8. ISELIN surface map' \#2. Refer to Table 6. 


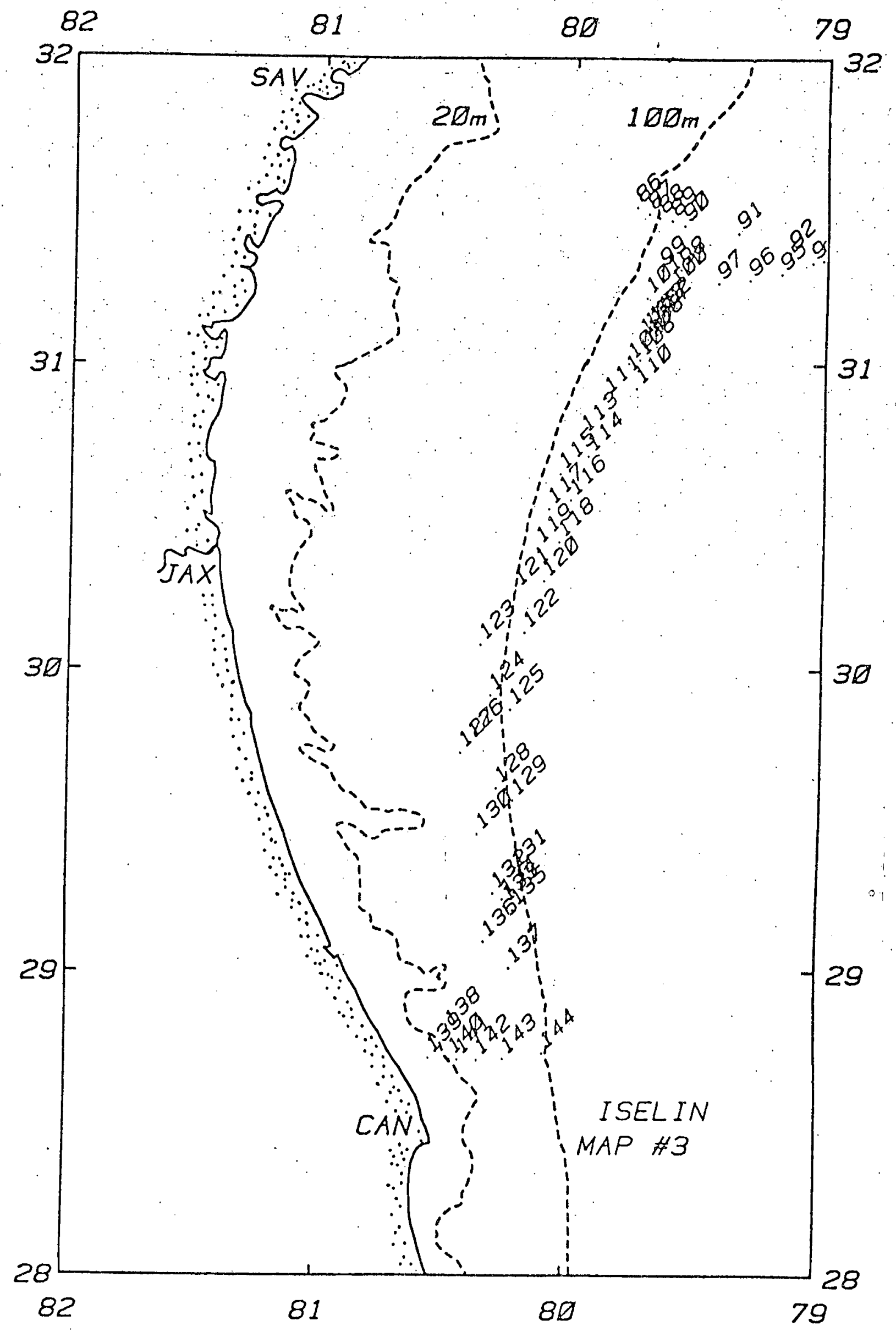

Figure 9. ISELIN surface map \#3. Refer to Table 6. 


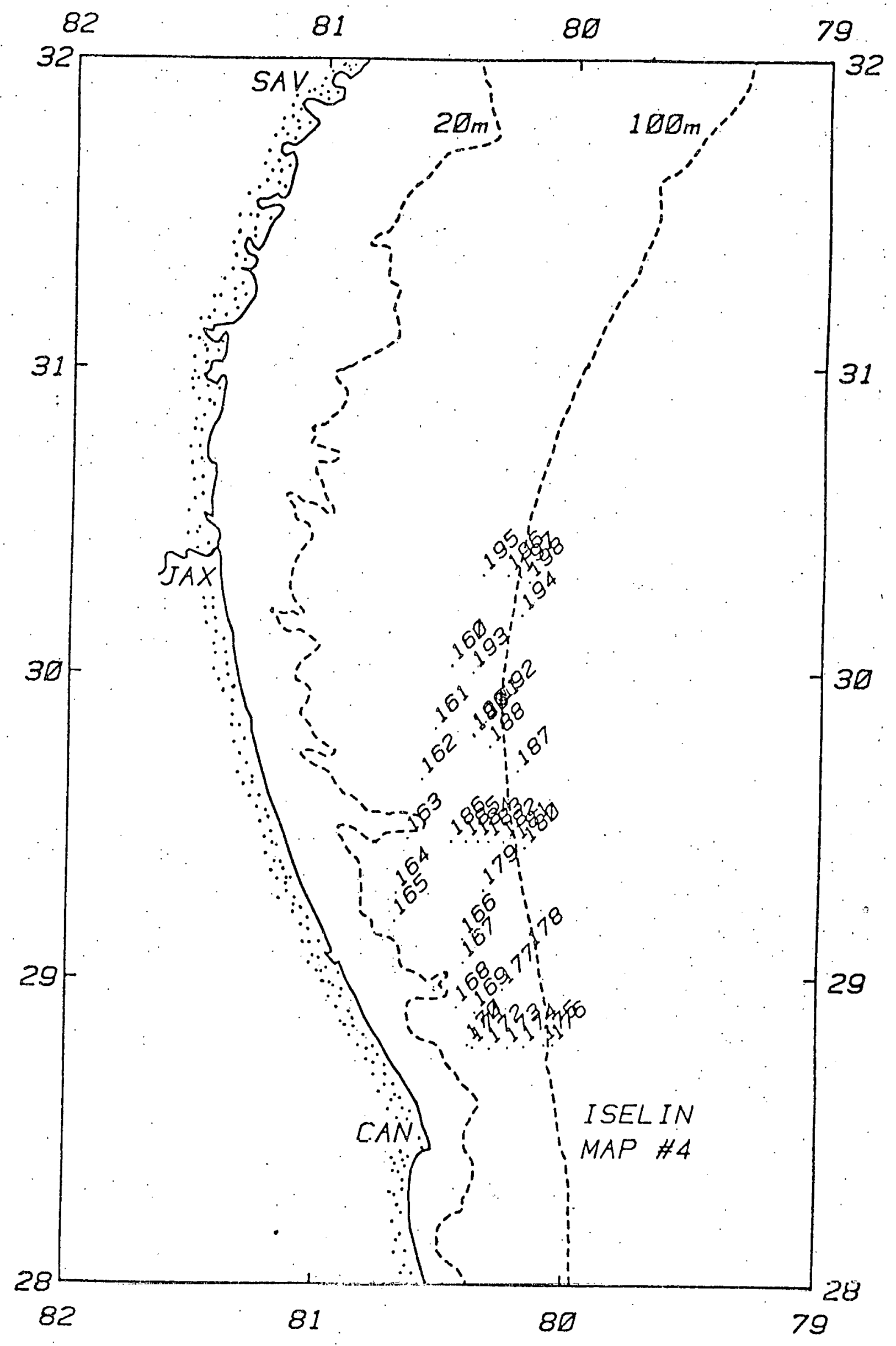

Figure 10. ISELIN surface map \#4. : Refer to Table 6. 


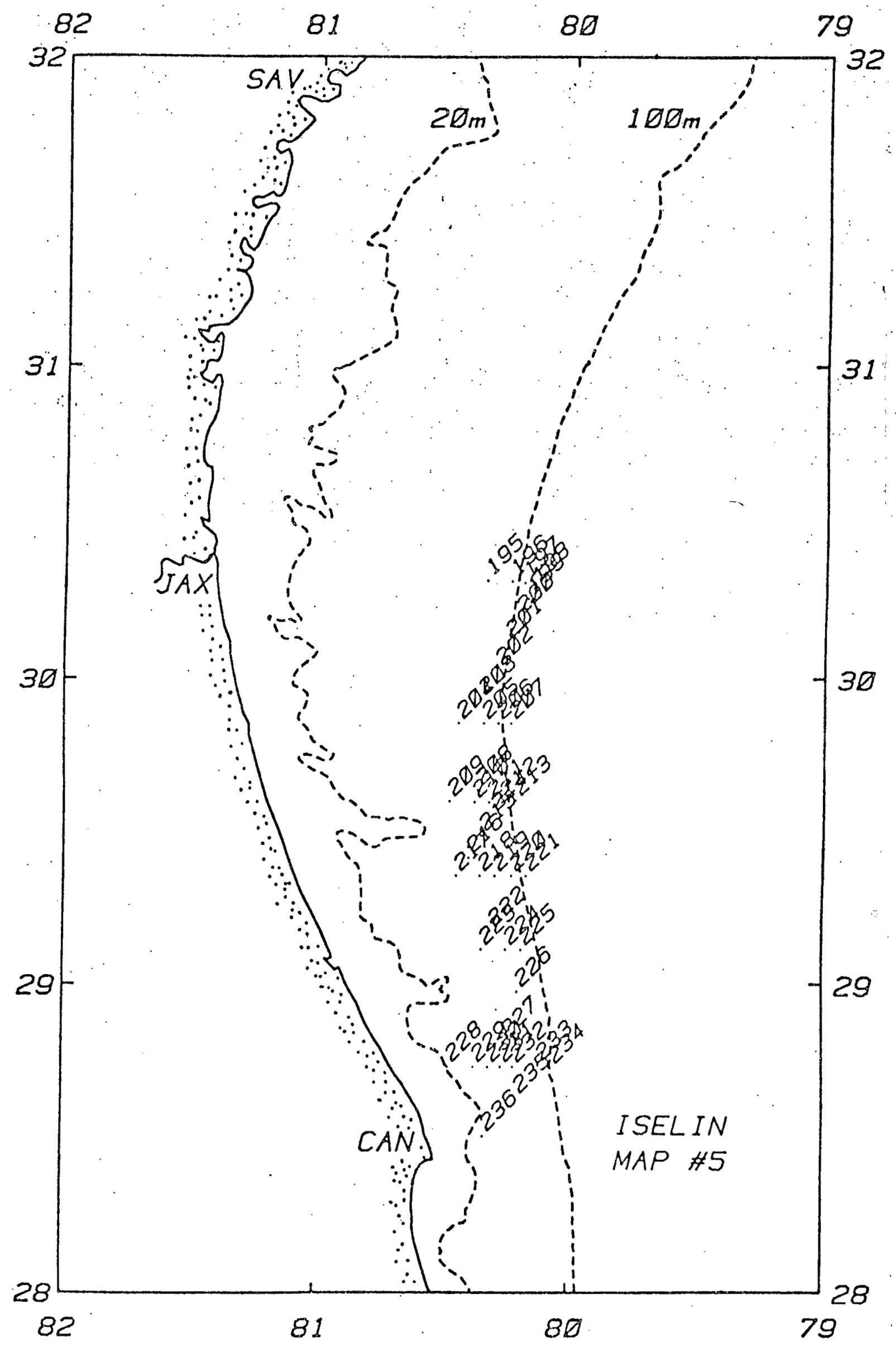

Figure 11. ISELIN surface map \#5. Refer to Table 6. 


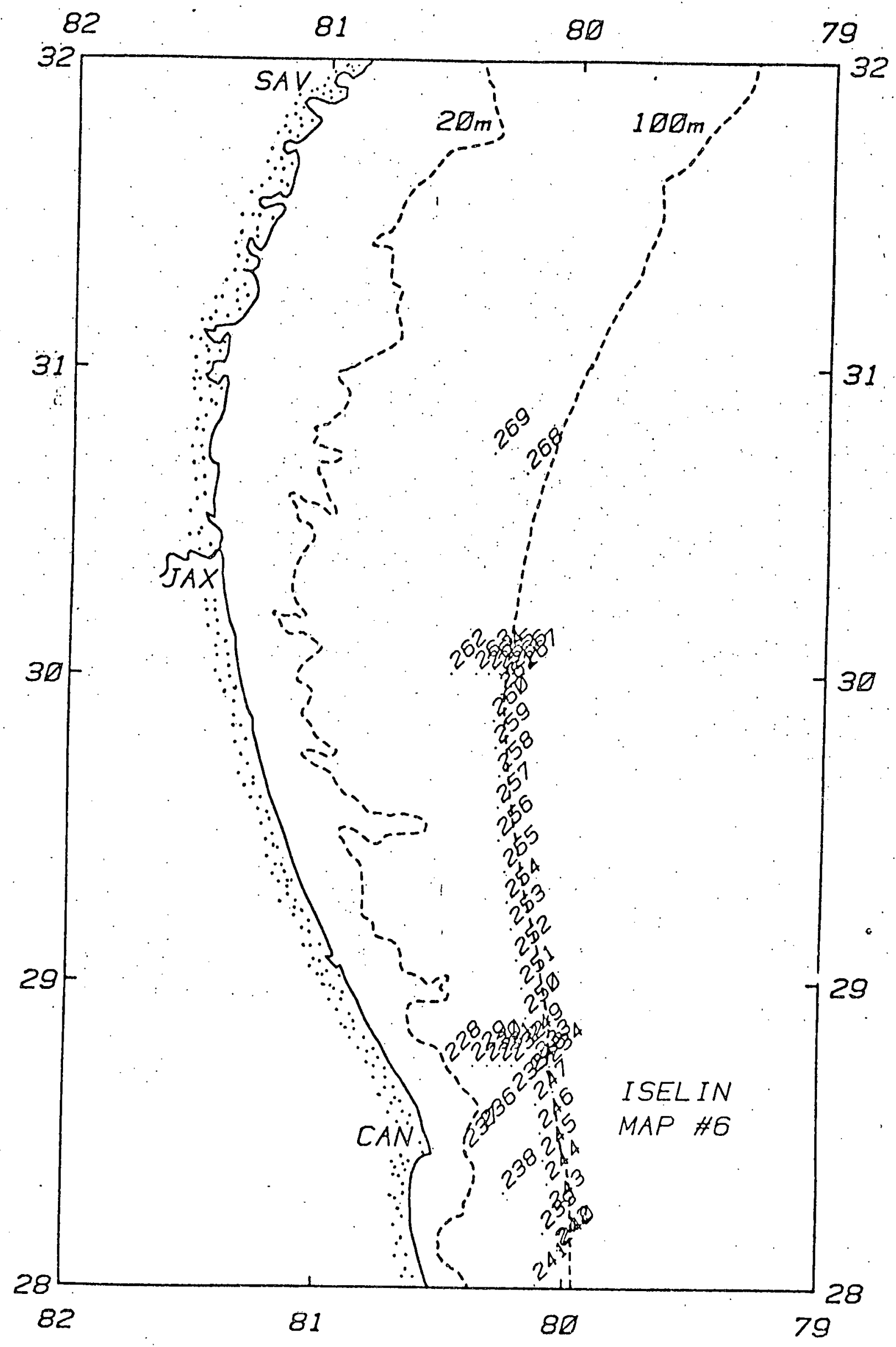

Figure 12. ISELIN surface map \#6. Refer to Table 6. 


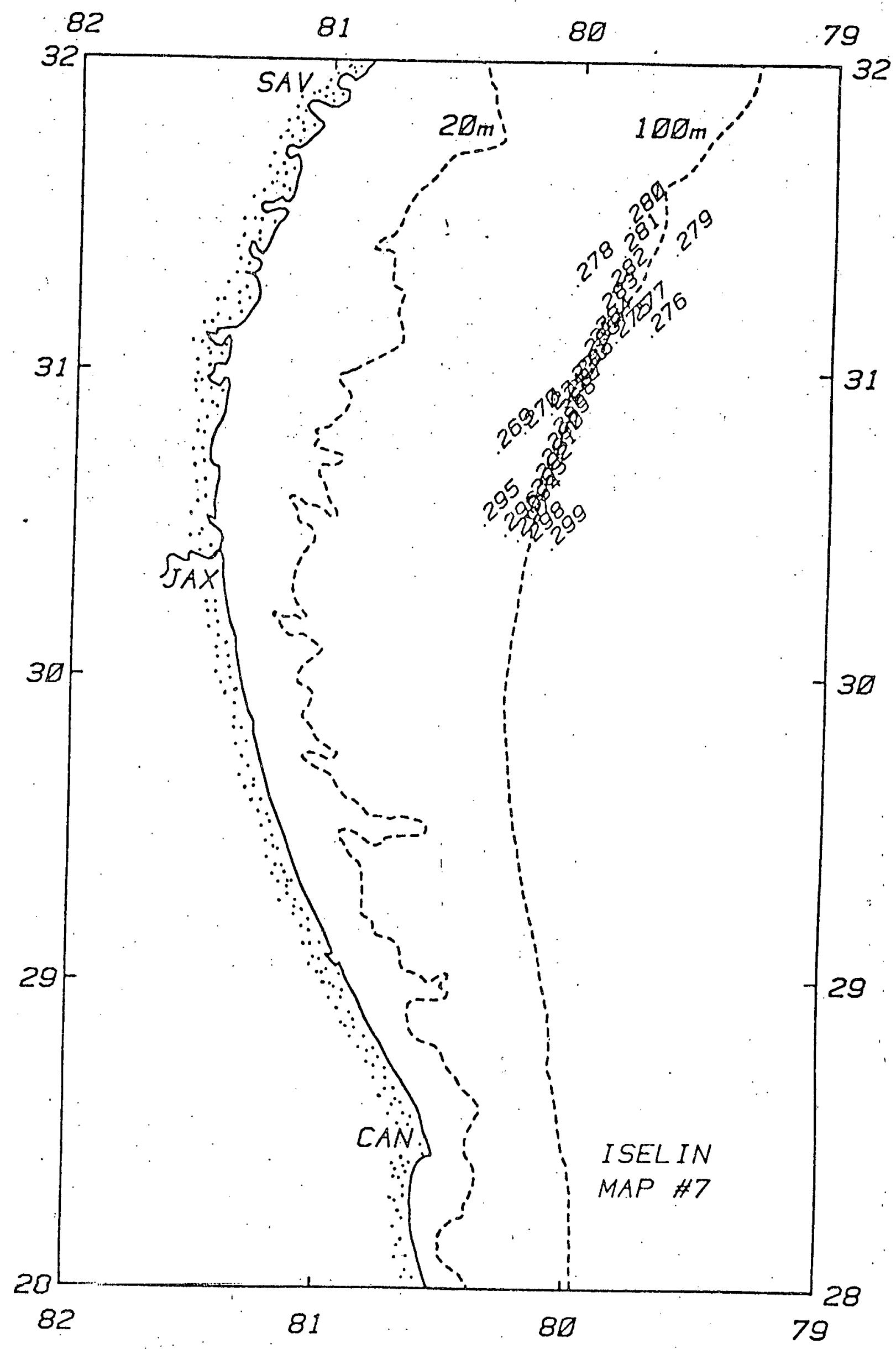

Figure 13. ISELIN surface map \#7. Refer to Table 6. 


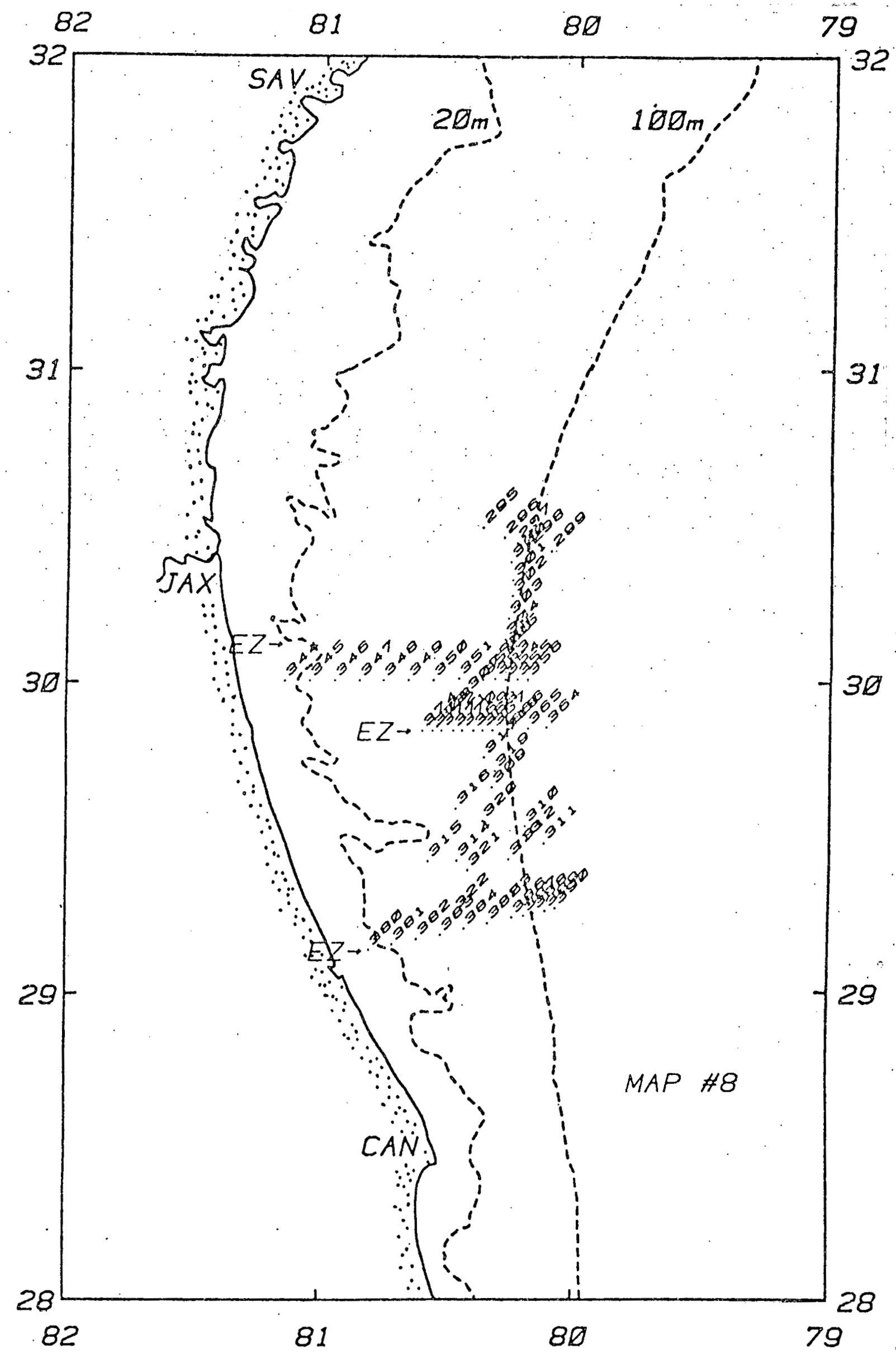

Figure 14. ISELIN surface map \#8. Refer to Table 6. EASTWARD sections indicated. 


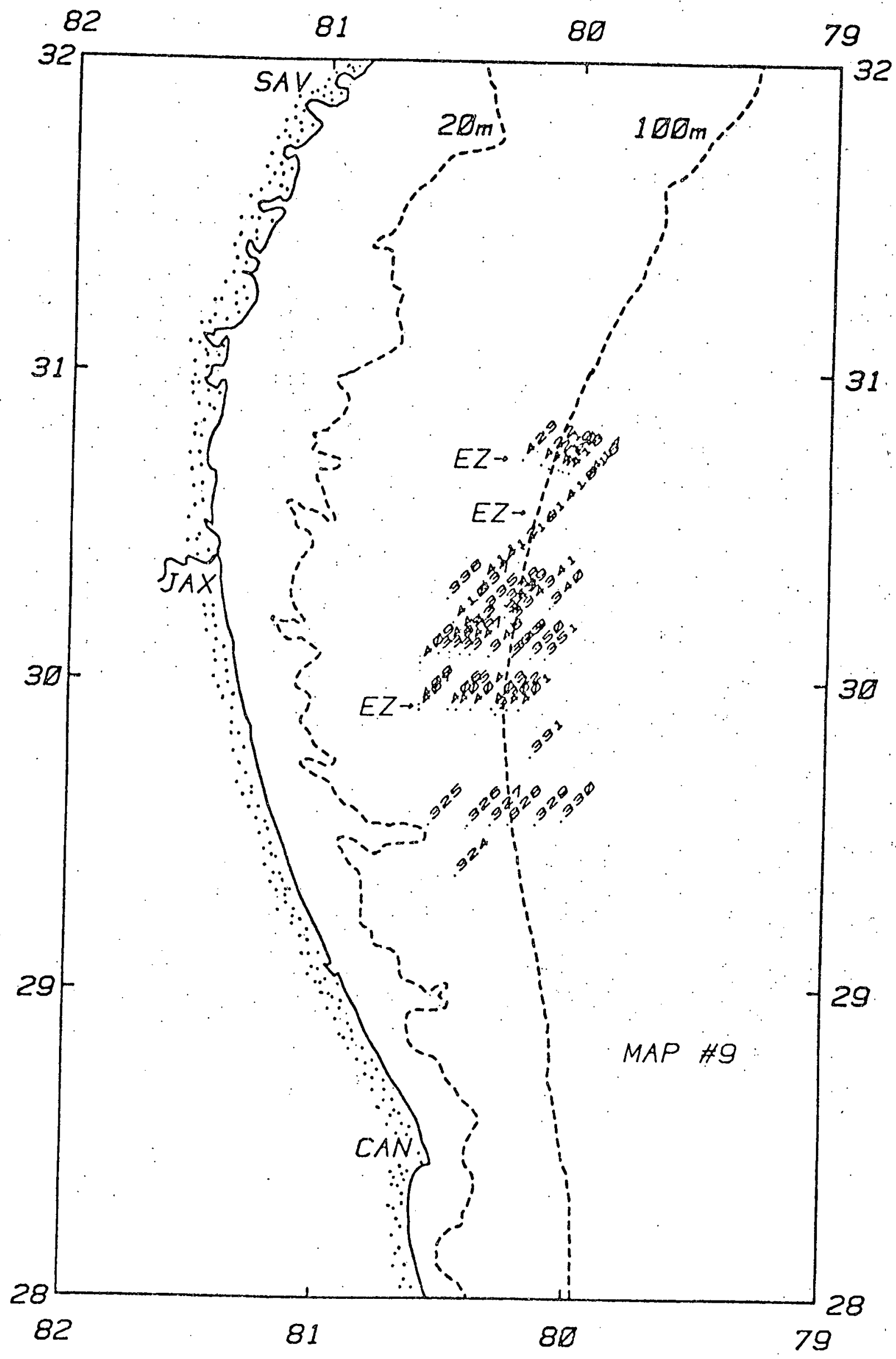

Figure 15. ISELIN surface map \#9. Refer to Table 6. EASTWARD sections indicated. 


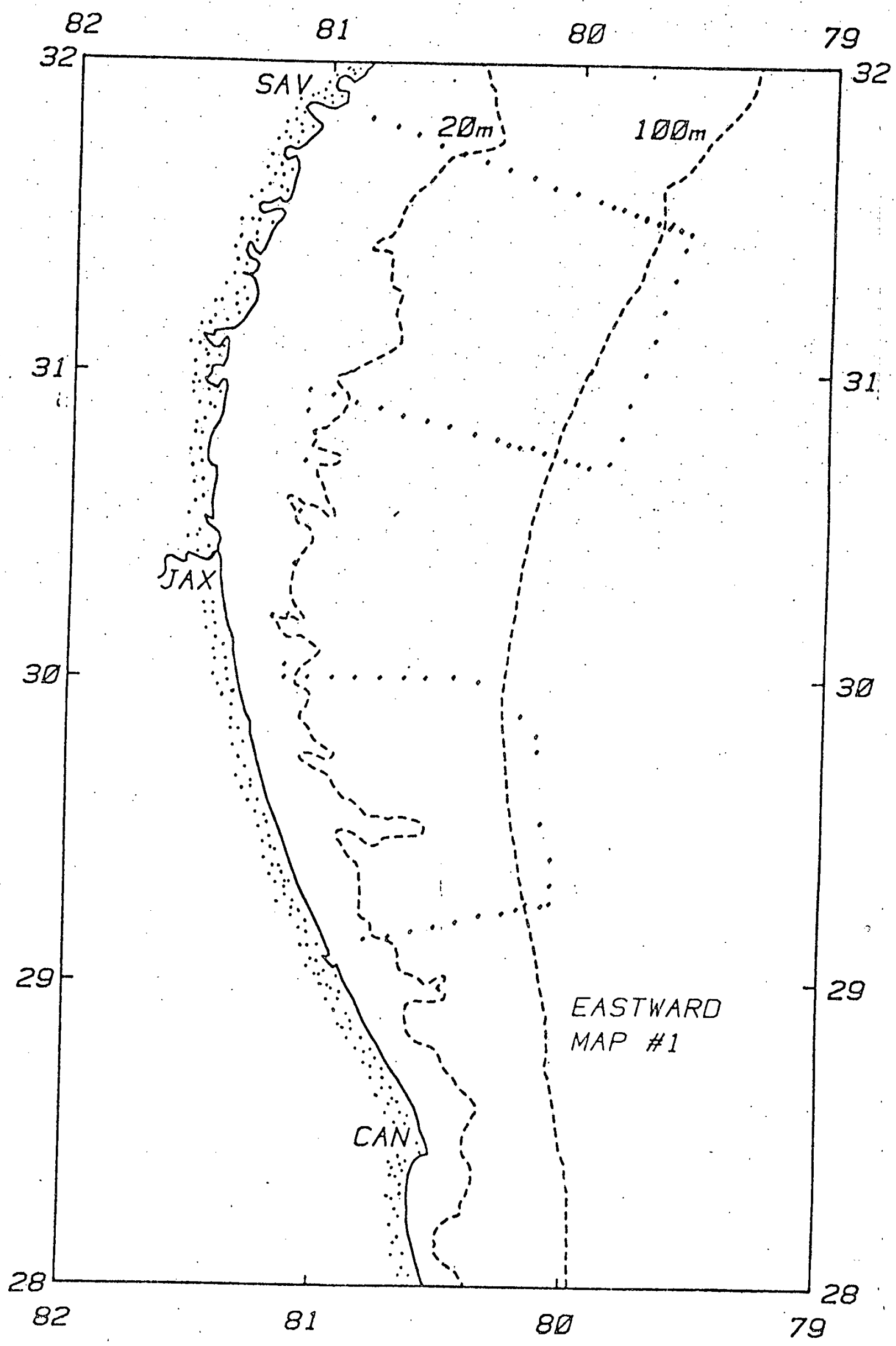

Figure 16; EASTWARD map \#1. Refer to Table 6. 


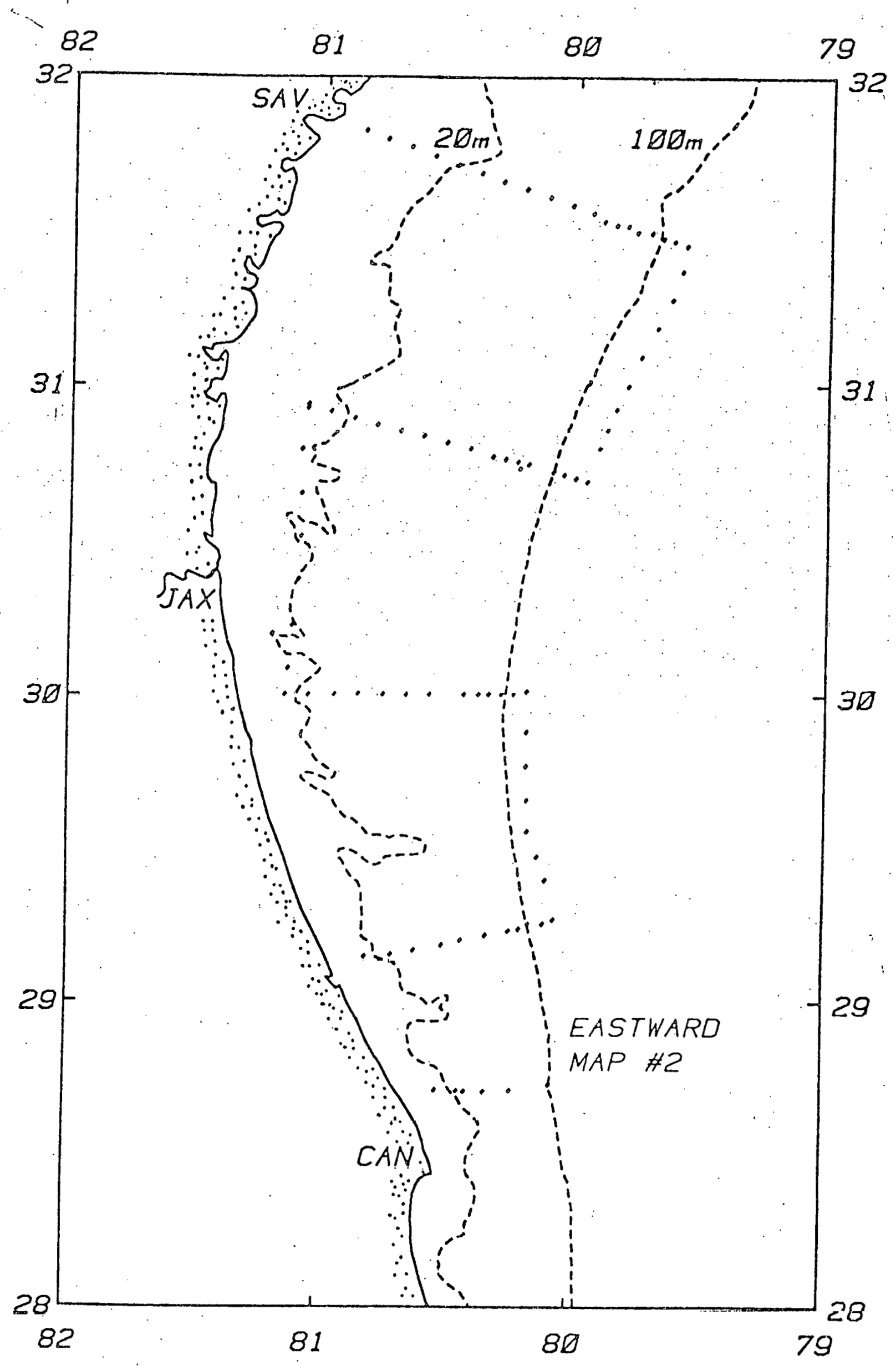

Figure 17. EASTWARD map \#2. Refer to Table 6. 


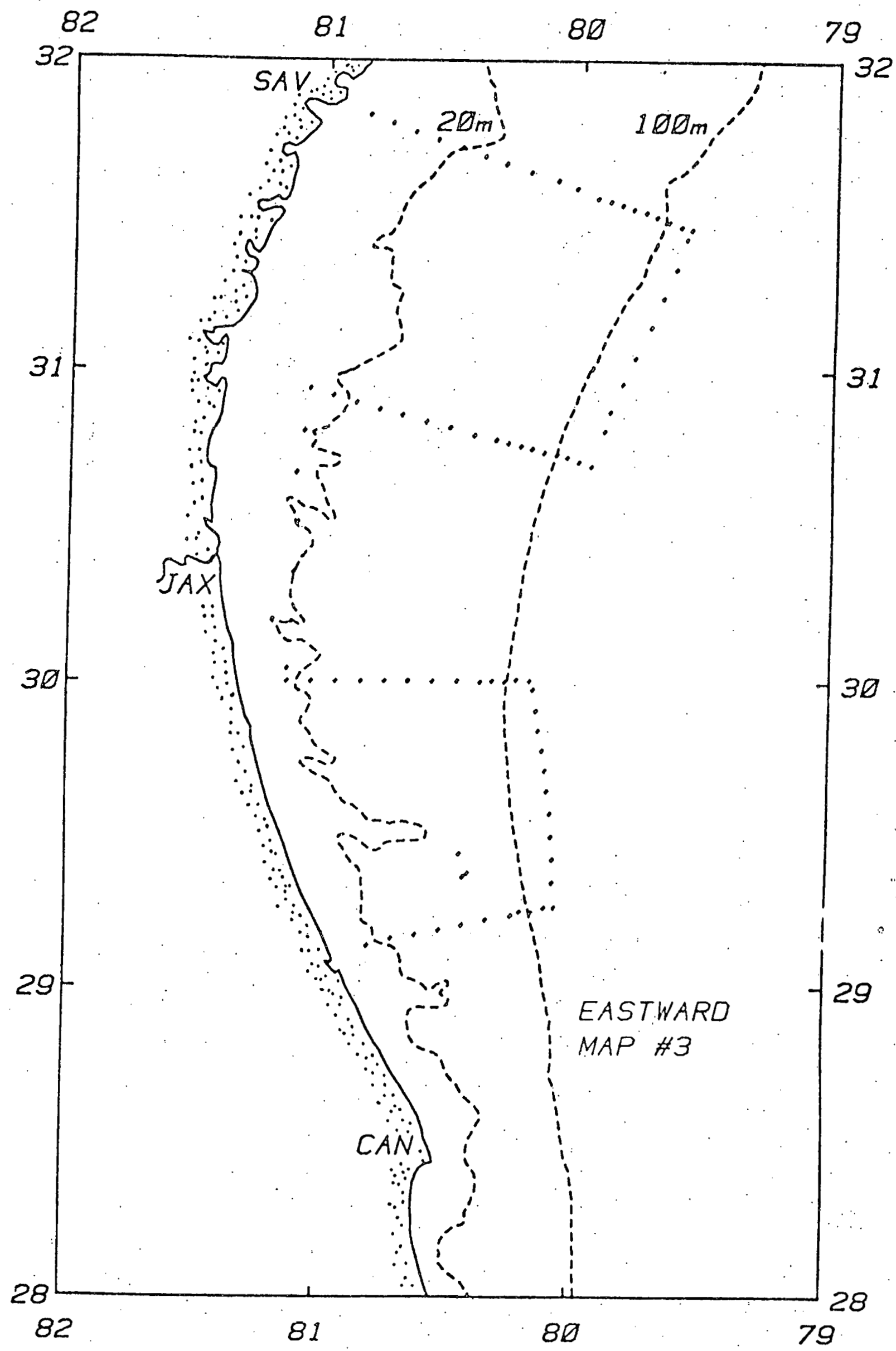

Figure 18. EASTWARD map \#3. Refer to Table 6. 


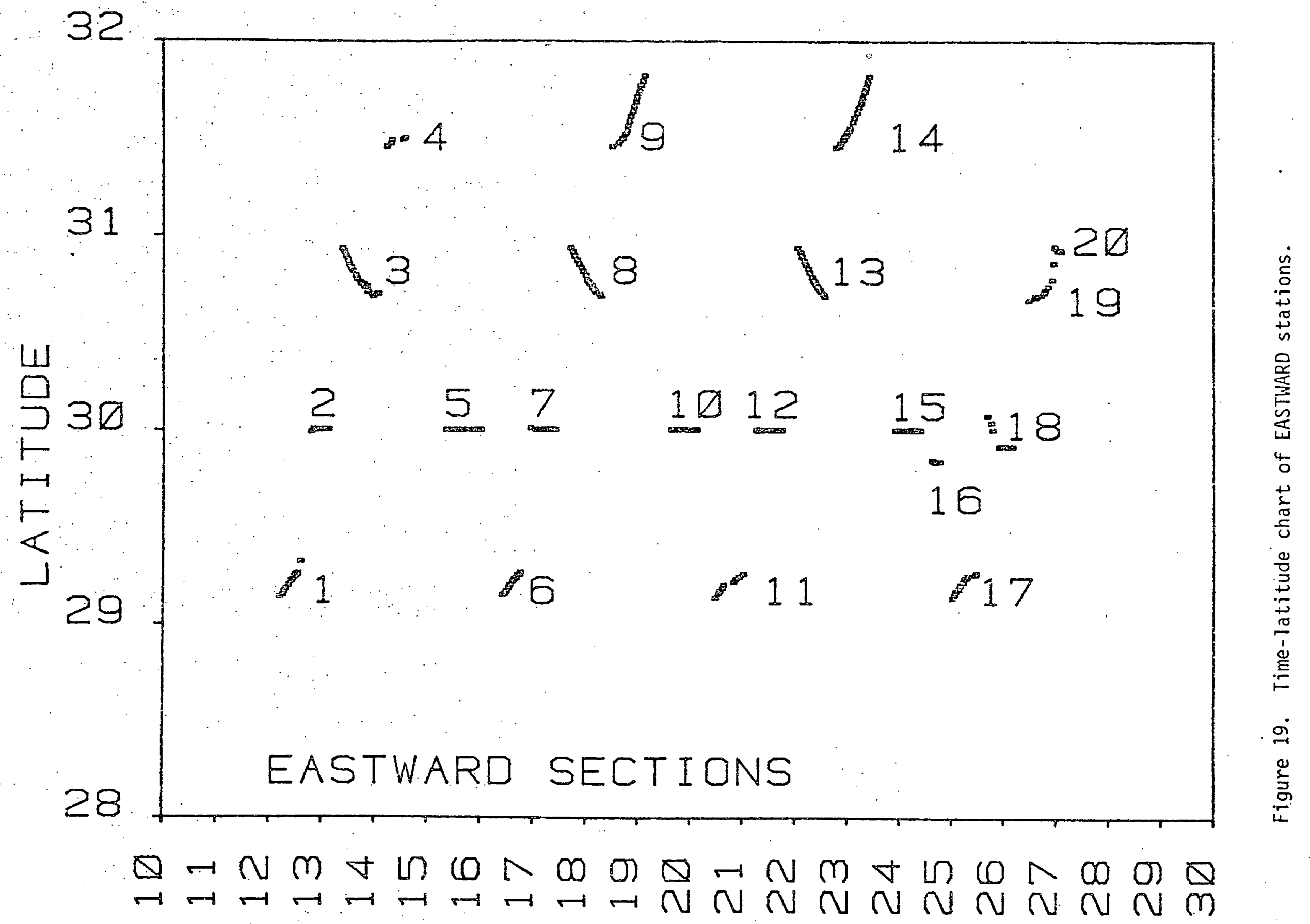

MAY IN IN APRTL $198 \square$ 


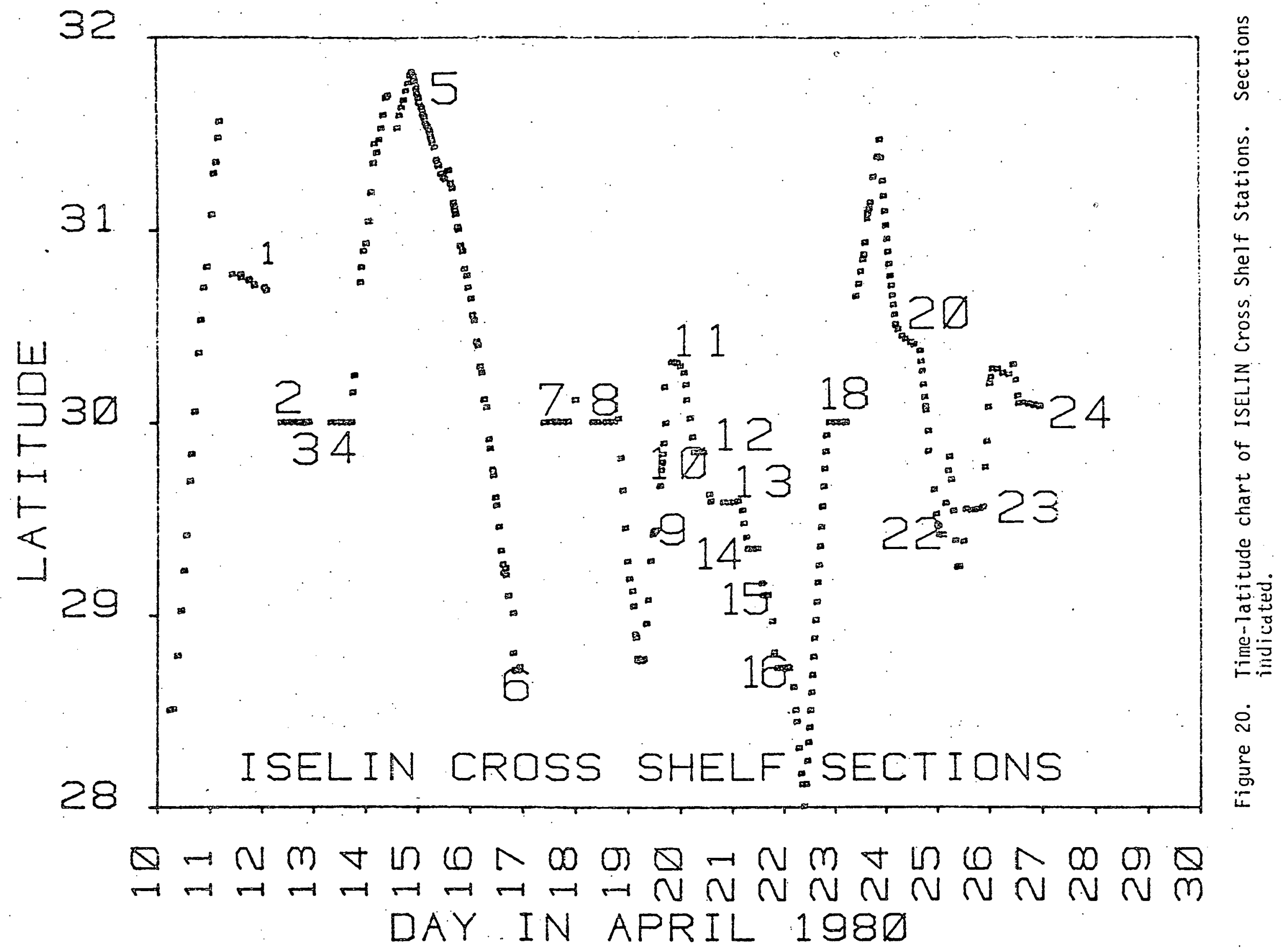

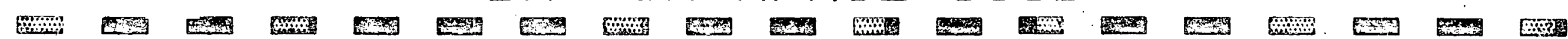




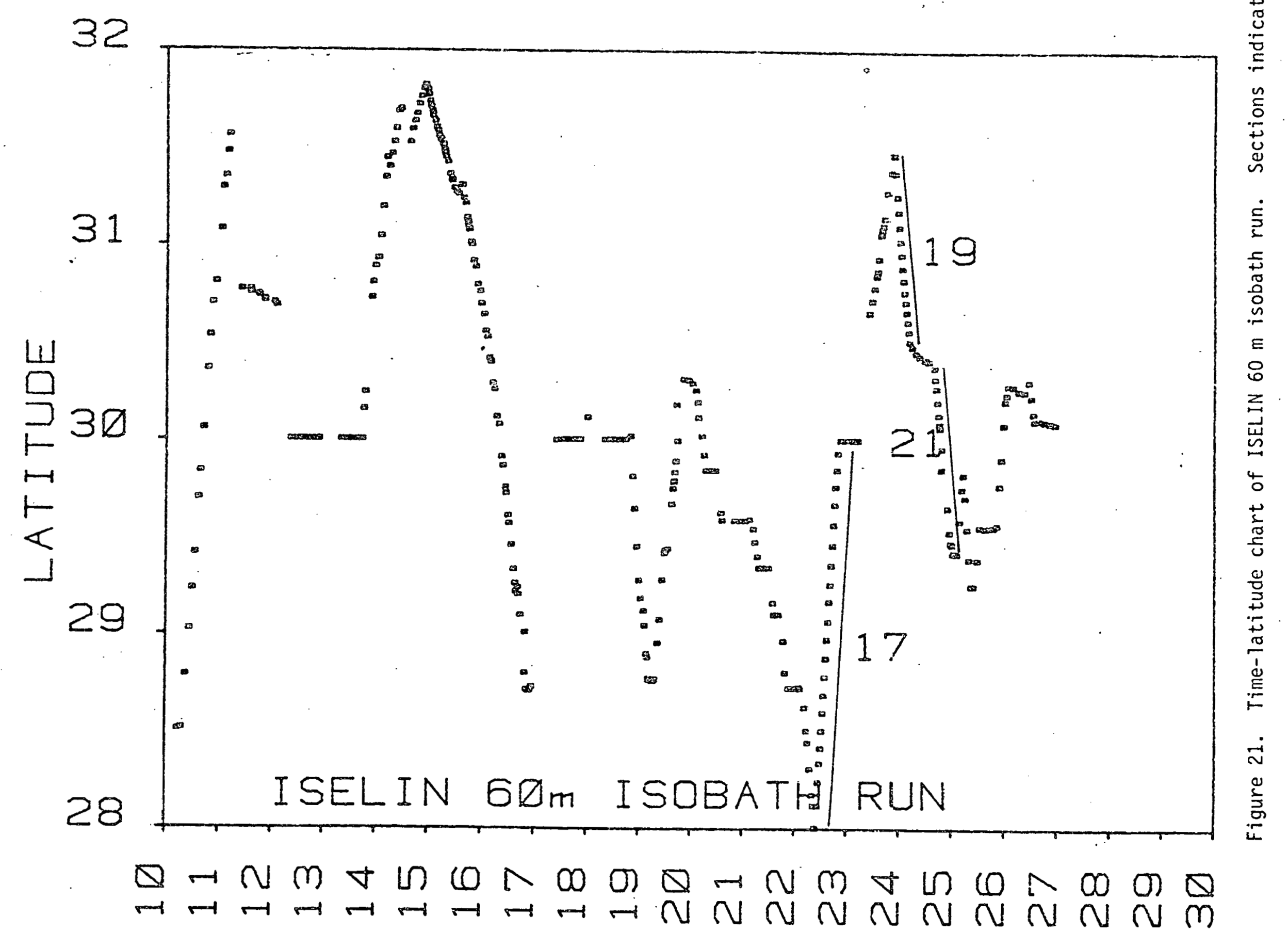
DAY IN APRIL 1980 


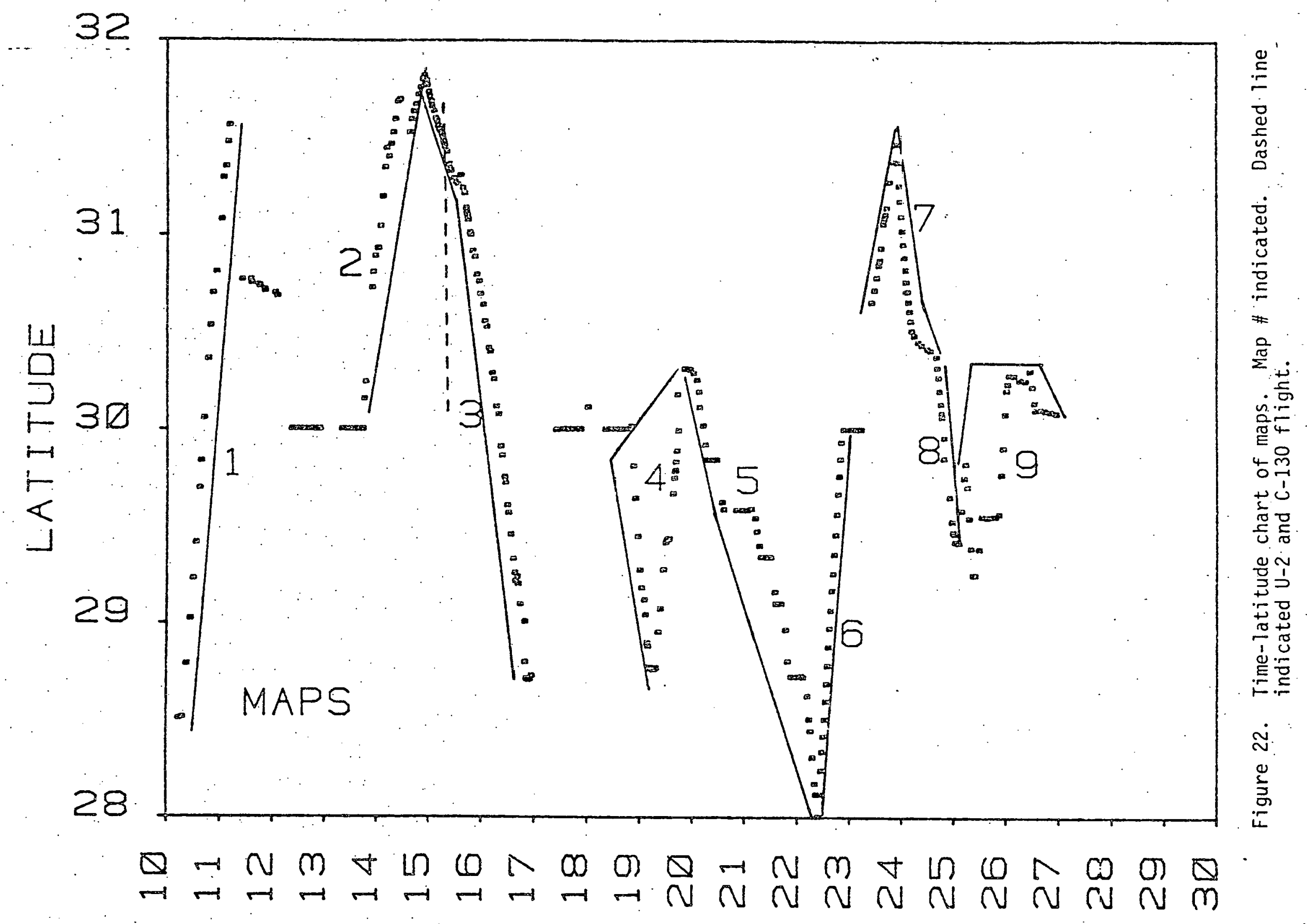

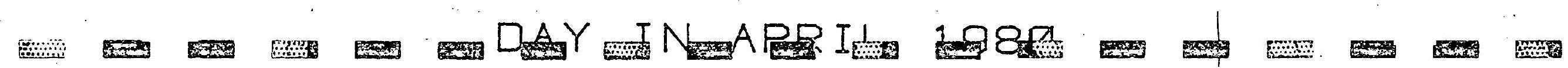




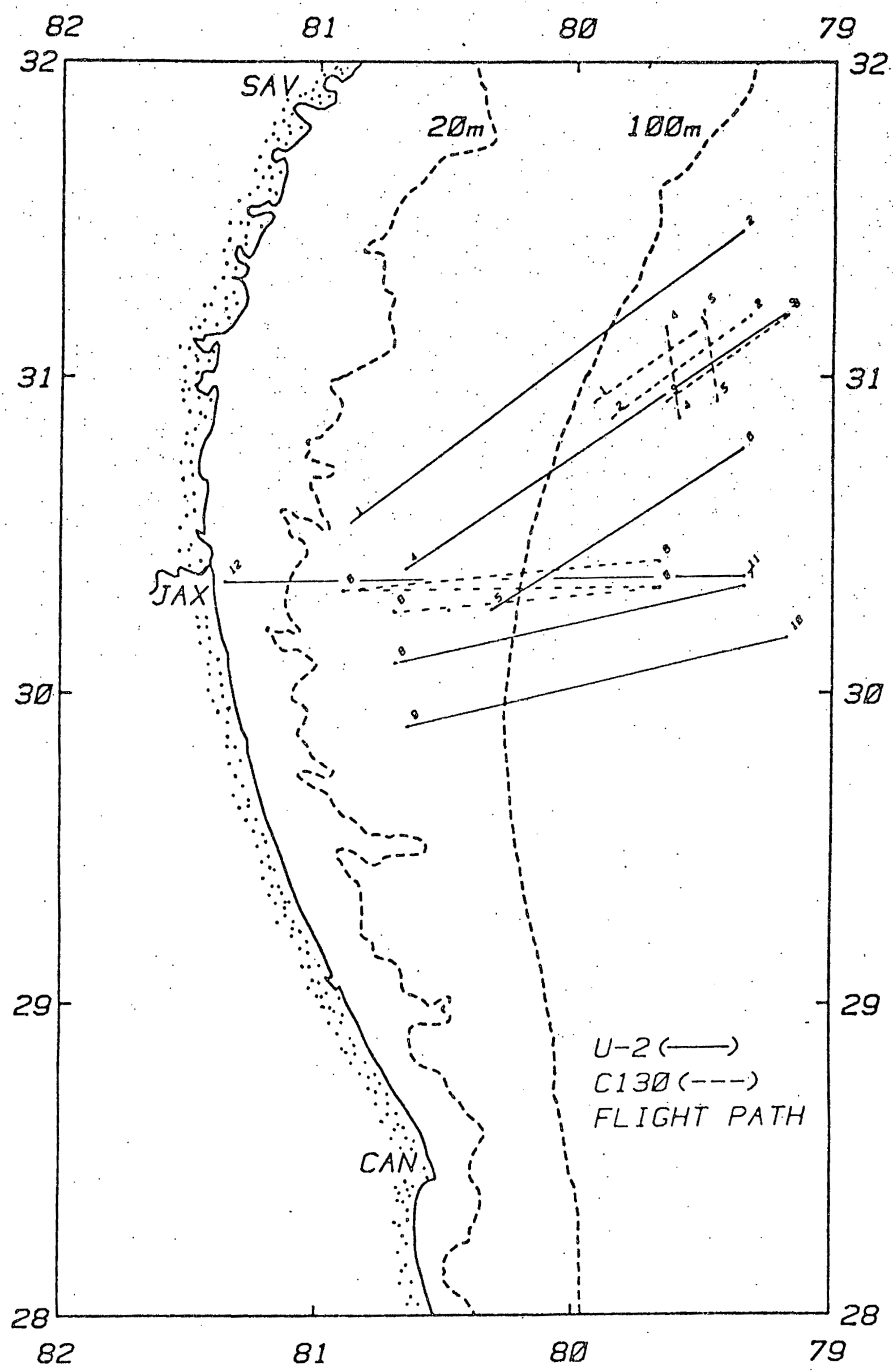

Figure 23. $U-2$ and $C-130$ flight track. Refers to Table $7 \& 8$. 


\section{Appendix 1}

ISELIN STATION LOG LIST 
The letter in the station name (column 2) refers to:

$$
\begin{aligned}
& c=\text { CTD COSt } \\
& x=X B T \text { Cost } \\
& h=\text { horizontal mapping run station } \\
& t=" \text { " " }
\end{aligned}
$$

ISELIN station numbers (column 1) and station name (column 2) are identical except for the added letter in the station name.

EASTWARD station names refer to standard grid locations when they are $c$ or $x$ stations. When station names are $t$ they refer to consecutive station numbers that coincide with the station number (column 1). 


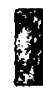

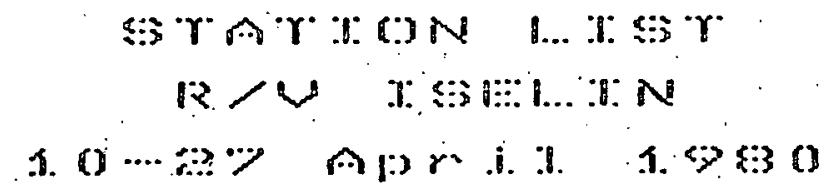


* STA. LAT LONG DATE EST Z(M) WUD SS WND WS B.P. AT W

1 In $28 \quad 30.08019 .5 \quad 10 / 4 / 80 \quad 0428$ $2 \quad 2 h$ 2. 30.480 1.0 $10 / 4 / 800600$

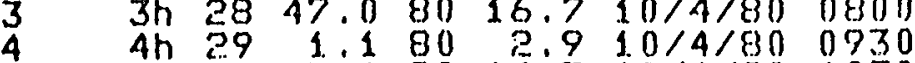
5 S Sh $29 \quad 13.6$ 80 13.5 10/4/80 1050

6

8

10

11

12

14

15

16

18

19
20

25. 21c $30 \quad 45.0830$ 1.3.0 $11 / 4 / 80 \quad 3.315$

22 22x $3044.58010 .011 / 4 / 801637$

23

24

26

$2 \%$

28
30
30

31
32
3

$\frac{32}{3}$

34

36

$3 \%$
38

38

40

41

$4 \frac{2}{3}$

44

45

46

$4 \%$

48

49
50

51. 5ih $30 \quad 48.57944 .7 \quad 13 / 4 / 80 \quad 2128$ 52. 52h 30 5. $5.579 .50 .7 \quad 13 / 4 / 80$ 20.31 53 53h 30 55.8 80 $0: 7$ 13/4/80 2330

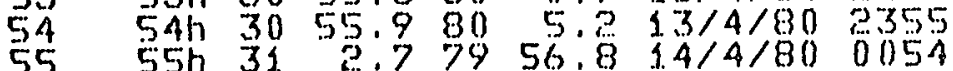

56 56h 31. $11.77947 .1 \quad 14 / 4 / 80 \quad 0202$

57

58

58

61

62

64

65

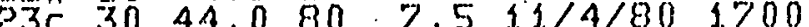

4. 0 1.1/4/80 1917

$26 \times 3043.07959 .0 \quad 5 / 4 / 800001$

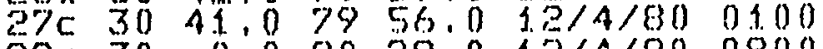
$\begin{array}{lllllll}2 B C & 30 & 0.0 & 80 & 28.0 & 12 / 4 / 80 & 0800\end{array}$

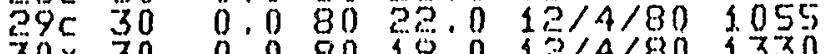

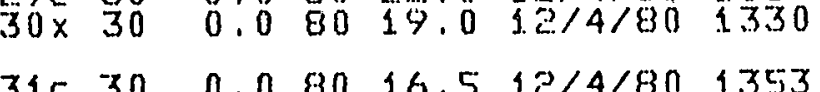
$32 \times 30 \quad 0.080 \quad 33.513 / 4 / 801630$

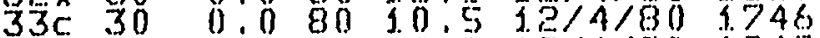
$34 \times 30 \quad 0.0 \quad 80 \% .932 / 4 / 8301849$ $36 \times 30 \quad 0.0 \quad 80 \quad 1.3 .7 \quad 12 / 4 / 80 \quad 1947$ $37 \times 30$

$38 \times 30$

$39 \times 30$

0.080 19.0 $12 / 4 / 802013$ 0.080 2:.0 $12 / 4 / 302029$ $0.080 \quad 20.0 \quad 12 / 4 / 802059$

41. 30

$0.0 \quad 80 \quad 28.013 / 4 / 80 \quad 0715$

$43 \times 30$

$0.0 \quad 80225 \cdot 0 \quad 13 / 4 / 8009$

$44 C \quad 30$

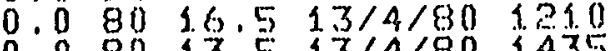

$46-30$

$0.0 \quad 80 \quad 10.5 \quad 13 / 4 / 80 \quad 145 \%$ $47 \times 30 \quad 0.0 \quad 30$ 2.0 $13 / 4 / 80$ 1642.

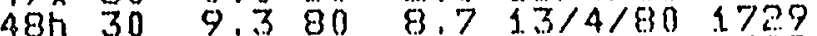
$49 h \quad 30 \quad 31.6 \quad 80 \quad 15.5 \quad 13 / 4 / 80 \quad 3808$

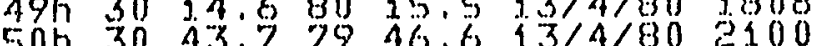
$\begin{array}{llllll}5 \% 7 & 31.20: 8 & 79 & 37.5 & 14 / 4 / 810 & 0303\end{array}$ $58 h \quad 31 \quad 26.8 \quad 79 \quad 31.1 \quad 14 / 4 / 800340$ $59 h$ 31 24.1 79 22.3 $14 / 4 / 800448$ $60 \mathrm{H}$ 31 $28.07930 .214 / 4 / 800545$

6.h 31. $31.67939 .25 .4 / 4 / 80 \quad 0645$ 6ih 31 35.7 $79 \quad 47.9 \quad 14 / 4 / 8300741$ $64 \mathrm{~h} 3141.77946 .811 / 4 / 800945$ $65 h$ 3j $31.87937 .3 \quad 14 / 4 / 80 \quad 1405$
$4 \times 3043.080$ $63 \mathrm{~h} 31.41 .07957 .3 \quad 34 / 4 / 800850$
$999305 \quad 3 \quad 305$

$\begin{array}{llll}999 & 305 & 4 & 300 \\ 999 & 305 & 4 & 300\end{array}$

$999 \quad 305 \cdot 4315$

99931.0

4320

999.310

$999 \quad 315$

$999 \quad 315$

4320

4 2.5

3330

999320

3165

$999 \quad 125$

999200

999200

2130

999210

2405

$\begin{array}{ll}2 & 235 \\ 2 & 230\end{array}$

999220

2 235

999 23.5

40 200

3
2
2 32

40256

44250

45300

125

1. 10

$2 \quad 10$

ง. 40

i. 110

$\begin{array}{llll}12 & 14.0 & 68 & 0 \\ 12 & 14.8 & 69 & 0 \\ 15 & 15.2 & 67 & 0 \\ 16 & 15.3 & 70 & 0 \\ 13 & 16.0 & 70 & 0\end{array}$

14

16.

$\begin{array}{ll}70 & 0 \\ 67 & 7 \\ 68 & 0 \\ 66 & 0 \\ 72 & 0\end{array}$

245 i. 10

$\begin{array}{ll}275 & 145 \\ 40 & 145\end{array}$

11.0

$48 \quad 140$

3 2. 145

3150

$6.83 \quad 45$

3150

162150

41.65

$260 \quad 3 \% 0$

41.60

400
260
260

4145

160160

4.45

$68 \quad 160$

$50 \quad 160$

4340

42150

4145

41.55

41. $160 \quad 4165$

46) 160

$48 \quad 160$

4160

61160

4160

$1601 \% 0$

4150

500350

$999 \quad 150$

$989 \quad 140$

999150

4150

4150

4160

99930

$999 \quad 150$

999350

$999 \quad 150$

5155

兵 155

5155

998160

195

$999 \quad 165$

4
5 165

$999 \quad 165$

999160

5165

5160

999 1.60

5160

999160

999365

$999 \quad 175$

5260

6205

b. 250
13

$\begin{array}{rrrr}10 & 16.0 & 66 & 0 \\ 18 & 16.0 & 67 & 0 \\ 18 & 16.0 & 67 & 0 \\ 6 & 18.8 & 68 & 0 \\ 5 & 21.0 & 72 & 1\end{array}$

$4 \quad 20.0 \quad 771$

7

6

6

8
12
12
15
15

15
15
15
20

20

20
23
23
20
20

12

15

14

15

15

15
15
15
15
25

32

54

24

$\frac{18}{2}$

4.8680

$15.0 \quad 680$

15.0 66 0

$15 \cdot 6 \quad 670$

18.9771

20.0 75. 1

$20.0 \quad 711$

$39.4 \% 21$

$18 \cdot 3 \quad 741$

$\begin{array}{lll}1.8 \cdot 3 & 74 & 1 \\ 17.8 & 72 & 1\end{array}$

$17.6 \quad 721$

$15.0 \quad 741$

$\begin{array}{lll}15 \cdot 4 & 72 & 2 \\ 15 \cdot 7 & 74 & 1 \\ 15 \cdot 7 & 74 & 1 \\ 15 \cdot 5 & 73 & 1 \\ 15.6 & 72 & 1\end{array}$

14.8721

$14.7 \quad 741$

3.4.0 72 1

13,8 72:

11.5721

1. 0.0 .701

$9.6 \quad 7751$

10.1 772

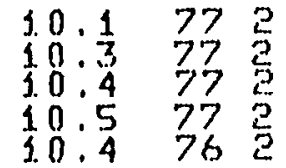

$\begin{array}{rrr}10.0 & 76 & 2 \\ 9.5 & 77 & 2 \\ 9.0 & 77 & 2 \\ 7.6 & 75 & 2 \\ 7.2 & 75 & 2\end{array}$

18

7.0 .732

1.9

$6.8 \quad 73.6$

$\begin{array}{llll}30 & 6.0 & 69 & 6 \\ 18 & 7.4 & 68 & ?\end{array}$
$98 \% 1.75$

$3.9 .0 \quad 761$

$15.6 \quad 7 \frac{4}{2}$ 
page 2

66 $66 h \quad 31.35 .6 \quad 79 \quad 16.6 \quad 14 / 4 / 80 \quad 1500$

$67 \quad 67 h 3138.07958 .014 / 4 / 801600$

68 68h 3140.2 80 $10.014 / 4 / 80 \quad 1700$

$6969 \mathrm{~h} 31.43 .28022 .3 .1 / 4 / 801800$

70 7oh $3145.58034 .514 / 4 / 801900$

71 7ih $3148.080 \quad 45.7 \quad 14 / 4 / 802000$

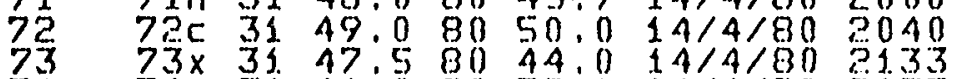

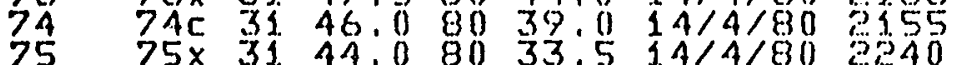

76

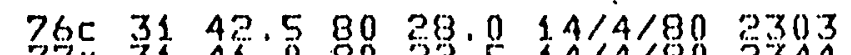

78

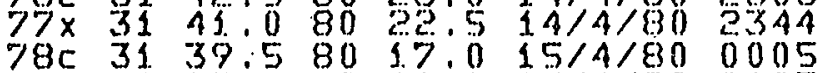

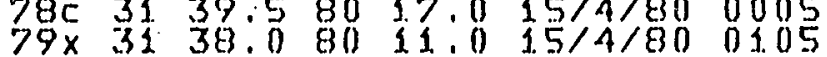

$8080 \mathrm{C} 31 \quad 36.0 \quad 80 \quad 6.0 \quad 15 / 4 / 80 \quad 01.46$

$8181 \times 31.35 .0 \quad 80 \quad 0.5 \quad 15 / 4 / 80 \quad 0224$

82 82. $3133.57955 .015 / 4 / 800250$

83

$83 \times 3132.5795 \% .0 \quad 15 / 4 / 800330$

84

$84 \times 3132.07949 .515 / 4 / 800400$

85

$85 \times 31.34 .07946 .5 \quad 3.5 / 4 / 80 \quad 0445$

$86 \quad 86 \times 3130.57944 .0 \quad 15 / 4 / 80 \quad 0500$

$8787 \times$ 31 29.57941 .0 15/4/80 0516

$88 \quad 88 \times 3128.57938 .5 .15 / 4 / 8300532$

89

$89 \times 23138.079 \quad 35.515 / 4 / 8000406$

$9090 \times 31 \quad 27.0793 \% .5 \quad 15 / 4 / 800600$

91. 91h 31. $35.5793 .9 .5 \quad 1.5 / 4 / 800700$

9. $92 \mathrm{~h} 31.21 .8797 \% 0$ 15/4/80 0800

93

95

934
944
940

9

96

98

$95 \mathrm{~h} 31.17 .579 \quad 9.1 .15 / 4 / 80 \quad 1000$

99

96h 31. 16.379 16.6 $15 / 4 / 80$ 1100 $97 \mathrm{~h} 31.5 .77924 .25 / 4 / 80$ 1.200 $98 \mathrm{~h} 31.38 .479 \quad 33.3 \quad 15 / 4 / 80 \quad 1300$

100 1.00h 31 14.4 $7935.415 / 4 / 80$ 1.4.

101. s.0.h 31 1.2.8 $7941.4 \quad 15 / 4 / 80 \quad 1500$

100 10.h 31 8.379 $39.3 \quad 15 / 4 / 801540$

103 3.03h 31. $7.37940 .6 \quad 15 / 4 / 801556$

104 104h $31.6 .67940 .015 / 4 / 80 \quad 1603$

105 3.05h $316.07941,615 / 4 / 801619$

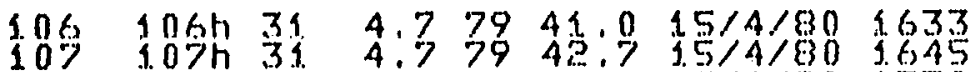

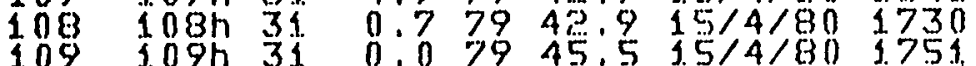

110 110h 3055.07943 .6 15/4/80 1840

11s. sish 3053.27954 .4 1.5/4/80 1941.

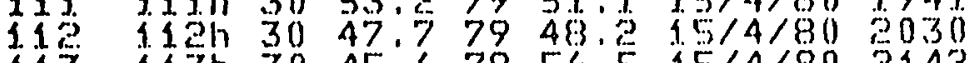

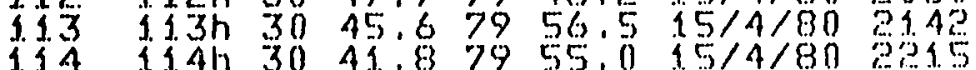

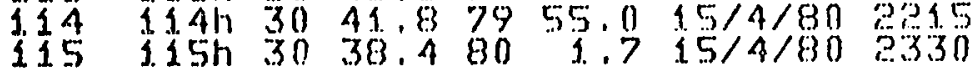

116 1.6h $30 \quad 33.37959 .1 \quad 16 / 4 / 800015$

1.7.3.7h 3039.6 80. $4: 3 \quad 36 / 4 / 80$ 0105

516 1.8h 30 25.1.80 1.8 $16 / 4 / 800200$

119 159h 30 23.9 80 7.1. $16 / 4 / 800240$

120 120h 30 17.3 80 5.3 $16 / 4 / 80 \quad 0332$

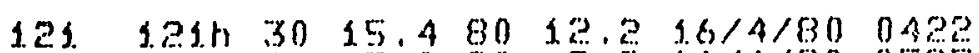

125 123h 30 7.0 80 $9.916 / 4 / 8005 \%$

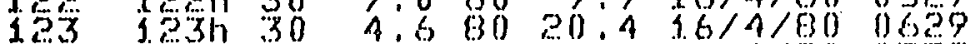

124 124h 2954.6 80 $17.9 \quad 16 / 4 / 8000738$

125 125h 58 51.8 80 13.2 $16 / 4 / 800814$

126 126h $2945.28023 .016 / 4 / 800925$

$999: 225$

999390

999190

999.190

999210

6225

5 2⿺辶万

4191

$4 \quad 19.0$

18
21
17
16

$7 \cdot 4$

681

998210

4220

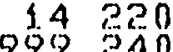

17260

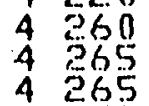

20260

4 2 270

30360

$\begin{array}{ll}37 & 270 \\ 38 & 570\end{array}$

4270

4285

$35 \quad 270$

4285

$40 \quad 270$

4295

$\begin{array}{r}43 \\ 998 \\ 9970 \\ \hline\end{array}$

$48 \quad 265$

4295

$\begin{array}{ll}5 & 2837 \\ 5 & 277\end{array}$

65265

5275
5278
5290

1. 48375

215275

300 200

$\begin{array}{ll}5 & 2870 \\ 5 & 2.70\end{array}$

990290

$\begin{array}{ll}5 & 292 \\ 5 & 295\end{array}$

999290

999270

5
5
5

$999 \quad 260$

5 260

999260

999260

999260

5260

998235

999240

999340

999245

$\begin{array}{ll}5 & 235\end{array}$

5246

5245

909240

999240

5240

909340

999250

999260

$\begin{array}{ll}5 & 240 \\ 5 & 240 \\ 5 & 2\end{array}$

999260

999260

(999 260

999270

4. 265

456

426

4280

999260

999260

5250
5284
5275
5275
5275

999290

909290

5307

999300

999315

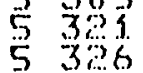

53 . 35

(2)

\section{0}

7.2

681

$7.5 \quad 679$

$7.8 \quad 661$

18
16
16
30
20

7.7 66 1

$8.4 \quad 661$

$9.3 \quad 6.41$

9.4641

10.7621

20
24
25
25

10.7

621

$1: 1.0$ हो. 1

1..8 60 1

11.8 60 1

20

12.5

571

36

26

12.5

1.3 .0

25
24
24
25
25

4.3 .2

1. 3.2

1. 4.0

1. 4.0

14.2

15. 2

38
21
20
20

3. $5: \frac{3}{3}$

15.8

15.9

\section{8}

j. 8

18

1.5. 8

1.5. 0

15,0

1. 4.4

20

21

1. 4.4

1. 2.6

1. 8

1. 2.8

1. 5.5

20

$1.2 \cdot 6$

15.8

ง.

1.3 .6

4.

14.5

1. 4.8

15.8

16.1

17.4

1. 7.4

3. 7.4

17.5

18.2

$58 \cdot 1$

501

581

581

61

641

681

66.

(2) 1

631

641

661

6,61

661

671

67.1

68.3

691

693

693

681

681

681

6) 1

64

641

18

19.

18

1.9 .5

641

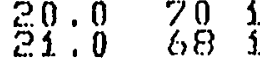

310

j. 5
18.0

$\begin{array}{lll}4 & 66 & 0 \\ 21 & 67 & 0\end{array}$

25.20

2... 1 6. 0

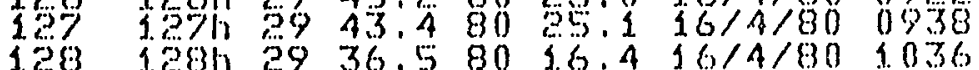

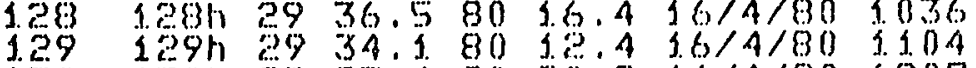

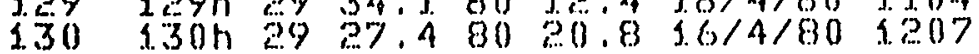

999315

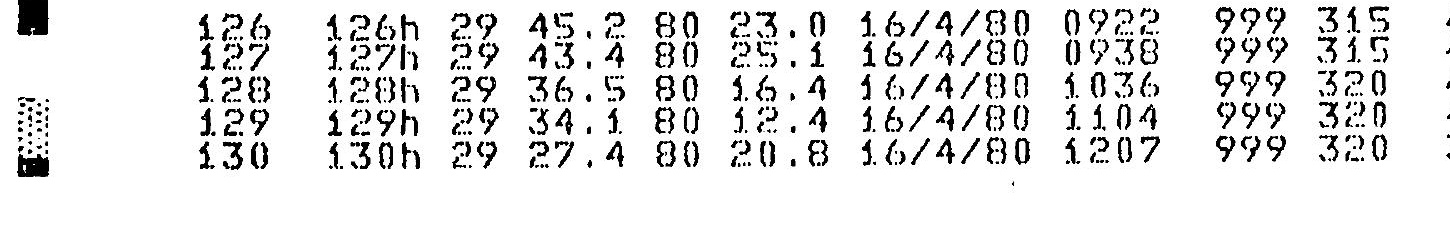


131. 131h 2919.9 80 13.0 $16 / 4 / 80$ 1312 132 132h 29 15.6 80 16.8 $16 / 4 / 80$ 1352 133 133h 29 13.0 80 14.1 $16 / 4 / 801415$ $131 \quad 134 h 29 \quad 14.4$ 830 $14.5 \quad 16 / 4 / 8301505$ 135 135h $2912.28012 .016 / 4 / 801527$

136 $136 \mathrm{~h} 29 \quad 5.8 \quad 8018.9 \quad 16 / 4 / 80 \quad 1621$ 137 137h 29 0.4 80 1. 7 $16 / 4 / 801833$ 338 1384 28 47.8 830 27.6 $3.6 / 4 / 80 \quad 3833$ $139139 \times 28 \quad 42.78032 .016 / 4 / 801915$ j. 40 140x 28 42.5 $8026.8 \quad 56 / 4 / 801940$

$1.41 \quad 141 \times 28 \quad 42.480 \quad 25.0 \quad 16 / 4 / 80 \quad 1951$ 142 3.2. $2842.38020 .316 / 4 / 802016$ $343 \quad 143 \times 2842.5$ 80 $14.016 / 4 / 802047$ $\begin{array}{llllllll}544 & 1.44 \times & 28 & 43.6 & 80 & 4.5 & 16 / 4 / 80 & 23.34\end{array}$

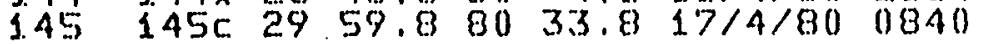

$146 \quad 146030 \quad 0.080 \quad 28.0 \quad 17 / 4 / 8013.30$ $1.4754 \mathrm{C} 30$.0.0 80 23.0 $17 / 4 / 801410$ $148348 \times 30 \quad 0.0603 .017 / 4 / 801444$ $149 \quad 149530.0 .0 \quad 80 \quad 16.5 \quad 17 / 4 / 80 \quad 3655$ 150 150x 30 0.0 80 13.5 $17 / 4 / 801996$

$151 \quad 1510 \quad 30 \quad 0.0 \quad 8030.5 \quad 17 / 4 / 80 \quad 1937$ 152 j52c 30 6.8 80 15.

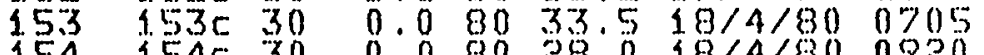

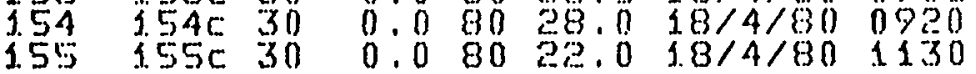

156 $1.56 \times 30 \quad 0.0 \quad 80 \quad 49.0 \quad 1.8 / 4 / 801328$ $15 \%$ 15.7 30 0.0 $8016.518 / 4 / 801340$

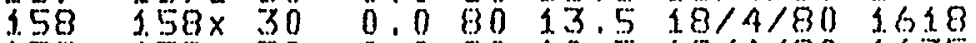
$15 \%$ 159C 30 0.0 90 50.5 $18 / 4 / 801635$

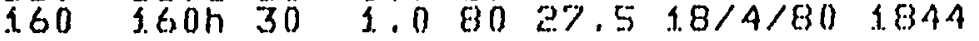

161. 161h $2948.68031 .518 / 4 / 80 \quad 1950$ 1.62 $362 h 2938.6 \quad 8034.7 \quad 18 / 4 / 800046$

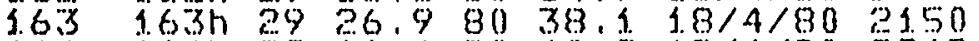
164 164h $2906.48040 .918 / 4 / 802549$ $165165 h 2910.98041 .3 \quad 18 / 4 / 802340$

166 j.66h $20907.1 \quad 80 \quad 29.8 \quad 19 / 4 / 800.100$ $16 \%$ 167h 29 2.5 80 2.7 $19 / 4 / 800128$ 1.68 1.68h 28 53.8 80 26.3 $19 / 4 / 800017$ 1.6\% 169h 2852.780 21.8 $19 / 4 / 800240$ 1.70 1.70h 28 46.2 80 2.3.6. $19 / 4 / 800.318$

1.71. 173 h $28 \quad 45.7 \quad 8023.3 \quad 19 / 4 / 80 \quad 0330$ $1.72 \quad 372 \times 28 \quad 45.48018 .28 / 4 / 800450$ $173 \quad 173 \times 2835.5 \quad 80 \quad 13.9 \quad 19 / 4 / 80 \quad 0510$ $174 \quad 374 x \quad 28 \quad 45.760$ \%.9 $39 / 4 / 80 \quad 0530$ $175 \quad 175 \times 2836.0 \quad 80 \quad 5.0 \quad 19 / 4 / 80 \quad 0554$

176 1.76h $28 \quad 46.0 \quad 80 \quad 2.9 \quad 19 / 4 / 800605$

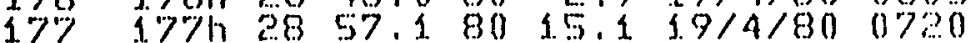

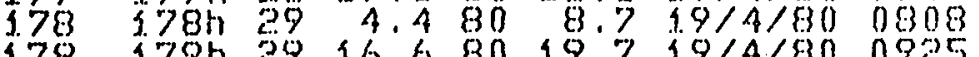

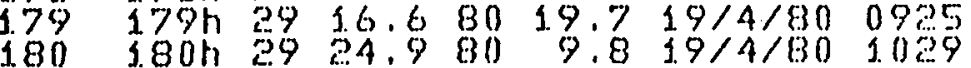

$181 \quad 181 \times 2925.580 .2 .019 / 4 / 801039$ 182 182x 28 25.9 30 15.0 $59 / 4 / 801053$ $183 \quad 183 \times 2926.0 \quad 8058.6 \quad 19 / 4 / 80 \quad 1111$ $184184 \times 2926.18021 .1 .9 / 4 / 80$ 1.1. $185185 \times 2926.180$ 2.4.0 $19 / 4 / 801138$

$186 \quad 186 \times 2926.28027 .6 \quad 19 / 4 / 801.55$ $18 \%$ 187x 2940.1 80 $11.6 \quad 19 / 4 / 80 \quad 1344$ $188 \quad 188 \times 5044.980 \quad 38.3 \quad 19 / 4 / 801420$ 1.89 $189 \times 2947.080$ 23.3.19/4/80 1440 190 190h

1.91. $191 \times 2949.98019 .4 \quad 19 / 4 / 80 \quad 1506$

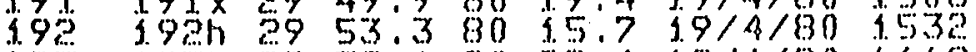
193 jơh 3959.680 20.4 $99 / 4 / 801619$

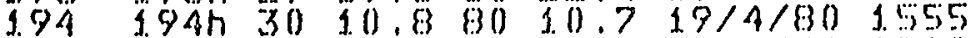
195 195030.18 .780 2.0.1. $19 / 4 / 801930$

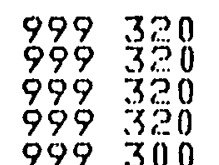

$\begin{array}{ll}3 & 300 \\ 3 & 320 \\ 3 & 350 \\ 3 & 320 \\ 3 & 301\end{array}$

12
12
12
12

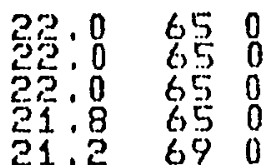

$999 \quad 350$

999

3350

$35 \quad 15$

$\begin{array}{rr}3 & 30 \\ 3 & 20 \\ 3 & 2\end{array}$

12
10
10
14
14

21.0

680

ว.

21:0

6680

$\begin{array}{ll}66 & 0 \\ 6 \% & 0\end{array}$

$24 \quad 2$

40 15

3

41270

3300

1.8
1.8
1.5
14

ㄹ.

690

21.3

690

23.0 671

$\begin{array}{ll}42 & 27 \\ 46 & 27\end{array}$

50 270

3300

10

23.

146 60

$3 \frac{3}{3}=0$

$288-75$

38

40110

48300

$3 \quad 385$

3325

1.

1. 0

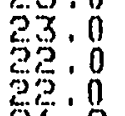

691

741

701

$4 \% 300$

331

67300

$990 \quad 345$

280315

331

3 .

10

1. 0

21.6

691

8

20.6

691

10

20.7. 70

10

10

11)

10

20.

20.5

19.4

721

$999 \quad 55$

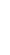

35

000130

99931.10

999 1. 05

3 .

3140

1.

10

j. 0

19.1701

999105

3115

999105

999105

9993.05

3 过

3 1.

3115

$999 \quad 105$

25310

36 1. 10

45 i. 10

3 1. 15

3 1.

3 115

6.3110

3 1. 15

9
9
10
10
10

3. 8

10

10

1.9.1

19.

19.2701

$19: 270$

$999 \quad 9$

9999

99990

3115

12

18.0

$1.7: 5$

(it) 1

$5 \quad 681$

17.4 68 1

9997

3160

3165

3165

8
14
8

14

17.4

17.0

17,0

16.8

1.6.8

16. 8

17.4

17.6

$1.7 \%$

17.6

88.70

$\begin{array}{ll}3 & 1.65 \\ 3 & 5.830\end{array}$

1

4

33

70

$\begin{array}{ll}3 & 180 \\ 3 & 180 \\ 3 & 280\end{array}$

1.7.

11.77

1. 1

1.7 .7

17.7

30 \%11 3250

1

54350

4645

$\begin{array}{lr}3 & 323 \\ 3 & 35 \\ 3 & 35\end{array}$

17.7

10

16.5

16.2

1. 6.2

681

690

69.1

703

701.

731

$7 \% 1$

$7 \frac{2}{4}$

743

74

74
74
1

741

741

701

902

8.2

15.8

1.6.2

16.0

703

70

68

681 
$196 \quad 196 \mathrm{C} 3018.680 \cdot 14.319 / 4 / 802045$ 197 197C 3018.3 .80 1. $1.219 / 4 / 8003143$ $198 \quad 198 \mathrm{C} \quad 30 \quad 17.380 \quad 9.1 \quad 19 / 4 / 802315$ $1.99199 \mathrm{~h} 3015.230 \mathrm{~g} 0.220 / 4 / 80.0050$ $200200 \mathrm{~h} 3011.5$ B0 13.6 $20 / 4 / 800145$

201 201h $30 \quad 6.880 \quad 15.1 \quad 20 / 4 / 80 \quad 0200$ $202302 h 30$ 1.180 $37.420 / 4 / 80 \quad 0345$ 203 203h $2955.28021 .320 / 4 / 800445$ 204 204c $2950.58026 .52014 / 800530$ 205 3050 $2950.58020 \% 520 / 4 / 800715$

$206 \quad 2066 \quad 29 \quad 50.5 \quad 80 \quad 17.0 \quad 20 / 4 / 80 \quad 0832$

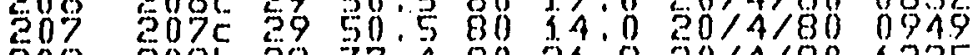
$208208 h 29 \quad 37.48021 .920 / 4 / 801235$ $20920962935.28028 .520 / 4 / 801305$ 210 210e $2935.0 .8020 .520 / 4 / 801850$

$\begin{array}{llllllllll}211 & 2110 & 29 & 35.0 & 80 & 19.0 & 20 / 4 / 80 & 2130\end{array}$

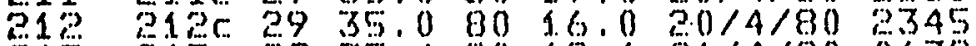
$213 \quad 2136 \quad 29 \quad 35.480$ 12.6 $21 / 4 / 800138$ 214 214h $29 \quad 32.5 \quad 80$ 1.8.1 $21 / 4 / 800335$ 215 215h 2928.4 .80 20.8 $23 / 4 / 80$ 0415

216 216h $2924.0 \quad 8023.8 \quad 21 / 4 / 80 \quad 0530$ 21. 218 318c $29 \quad 20.580$ 21.0 $31 / 4 / 800735$ $219 \quad 21902920.5$ 80 $37.421 / 4 / 800855$ $2202002020.58013 .521 / 4 / 800945$

221

22

204

225

20

2.28

230

231

23

234 234x $2843.08003 .525 / 4 / 800131$

$235 \quad 235 h \quad 28 \quad 37.280 \quad 1.1 .922 / 4 / 80 \quad 0.337$

236 236h $28 \quad 30.0 \quad 80 \quad 19.6 \quad 22 / 4 / 80 \quad 0431$.

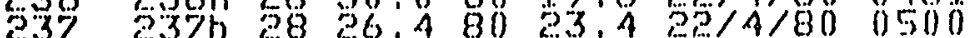
236 238h 28 18.2 80 1.4.5 $20 / 4 / 800600$ $239539 \mathrm{~h} 2810.280$ $5.020 / 4 / 800700$ 240

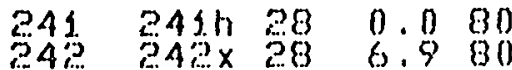
$340 h 286.980$

$0.825 / 4 / 80 \quad 0729$

6. $923 / 4 / 800819$

$243 \quad 243 \times 28 \quad 14.280$

$343.544 \times 2800.080$

$245 \quad 245 \times 28 \quad 24.780$

$1.923 / 4 / 800912$

$4.0 \quad 3 \% / 4 / 800090$

5.0 $20 / 4 / 80$ 1 055

246 $246 \times 2830.080 \quad 5.725 / 4 / 80$ 1.25

$247 \quad 247 \times 2835.680 \cdot 6.8 \quad 22 / 4 / 60 \quad 1355$

$248328 \times 2841.0 \quad 80 \quad \% .62 \% 4 / 80 \quad 1225$

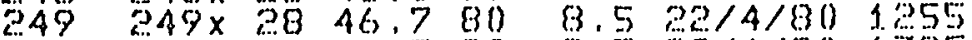

$250250 \times 2852.580 \quad 9.322 / 4 / 80 \quad 3.355$

251 $251 \times 28 \quad 58.3 \quad 80 \quad 10.4 \quad 22 / 4 / 80 \quad 1355$ 252 252x $29 \quad 3.98011 .723 / 4 / 801455$ $253 \quad 253 \times 29010.0 \quad 80$ 13.1. $22 / 4 / 801455$ $254 \quad 254 \times 289015.3 \quad 80 \quad 14.252 / 4 / 80 \quad 1525$

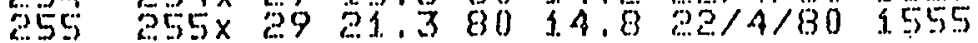

$256 \quad 256 \times 2927.4 \quad 80 \quad 16.322 / 4 / 80 \quad 1625$ 557 559x

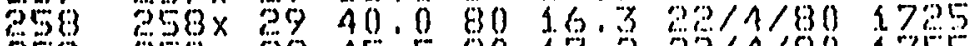

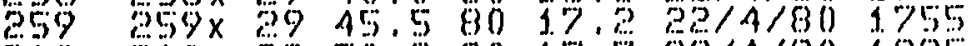
$260 \quad 260 \times 2950.880 \quad 17.7 \quad 3 \% / 4 / 801836$ $\begin{array}{rrrrrrrr}55 & 80 & 4 & 80 & 18 & 16.1 & 68 & 5 \\ 139 & 95 & 4 & 95 & 21 & 16.1 & 68 & 5 \\ 220 & 115 & 4 & 115 & 12 & 16.2 & 68 & 5 \\ 999 & 115 & 4 & 115 & 12 & 16.2 & 68 & 5 \\ 999 & 115 & 5 & 1.15 & 27 & 15.0 & 64 & 5\end{array}$

$999 \quad 115$

4115

18
18
13
10
12

$90 \%, 110$

$38 \quad 100$

47 1. 1.0

411.5

4305

$\begin{array}{ll}52 & 100 \\ 138 & 335 \\ 999 & 335\end{array}$

$999 \quad 335$

45340

4330

43.35

4 3 30

48340

5340

67340

$113 \quad 341$

5. 340

5340

बत्र 3 1

$999 \quad 315$

520

330

$9993 \% 0$

28 320

$40 \quad 340$

$46 \quad 320$

5310

71315

53.30

5 3:?

$11.8 \quad 31.5$

5315

5 उ००

$33 \quad 315$

48 34

67335

5300

$999 \quad 1$

(15

1.

2

$\begin{array}{rr}38 & 60 \\ 45 & 60 \\ 70 & 6 \\ 84 & 60 \\ 999 & 250\end{array}$

$\begin{array}{rr}4 & 1: 0 \\ 4 & 0 \\ 3 & 0 \\ 3 & 280 \\ 3 & 270\end{array}$

$\begin{array}{lll}15.0 & 64 & 2 \\ 15.0 & 64 & 2 \\ 15.0 & 64 & 2 \\ 15.0 & 64 & 1 \\ 15.0 & 66 & 1\end{array}$

$\begin{array}{llll}18 & 15 \cdot 1 & 69 & 1 \\ 20 & 15 \cdot 1 & 67 & 1 \\ 20 & 15 \cdot 0 & 68 & 1 \\ 20 & 14.4 & 67 & 1 \\ 18 & 13.8 & 66 & 1\end{array}$

$20 \quad 12.8 \quad 681$

20 13.8 681

20 $11.8 \quad 681$

$20 \cdot 11.6831$

1911.8 69

20 12:0 69

20) $12.0 \quad 701$

$23 \quad 12.4 \quad 681$.

$22 \quad 12.4711$

14 12.4 71

14 1..4 6831

999300

$999 \quad 310$

$999 \quad 345$

$990 \quad 345$

3245

$\begin{array}{ll}3 & 250 \\ 3 & 253\end{array}$

3525

3 2 50

330

3 3.

3350

3350

909330

$63 \quad 330$

6. 53

$63 \quad 325$

3340

3315

3
3
3
3

$\begin{array}{ll}3 & 355 \\ 3 & 3\end{array}$

$58 \quad 335$

6. 335

33.35

61335

$59 \quad 335$

33.35

3.335

6.3345

3315

62315

3315

603.35

$\begin{array}{ll}3 & 335 \\ 3 & 335\end{array}$

603.35

60300

3305

60) 280

3283

$69270 \quad 3269$

$6460 \quad 3 \quad 303$

$60 \quad 45$ 20 150

6045
1. 2

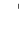

1.1.

1.1.

11.8

1.1. 6

1. 1,8

1.0

1. 1.

12.

4. 1.9

1.1. 8

11.5

10

1.3

13

魚

1.3. 1

1.3.

12

1.

13.

8

1.4 .3

1. 4.6

790

7.31

701

70 i.

741

711

671

है? 1

$\begin{array}{rrrr}9 & 14.7 & 74 & 1 \\ 9 & 14.7 & 74 & 1 \\ 10 & 14.7 & 74 & 1 \\ 10 & 14.7 & 74 & 1 \\ 10 & 14.7 & 76 & 1\end{array}$

$\begin{array}{llll}10 & 1.5 .0 & 78 & 1\end{array}$

1. $15.0 \quad 781$

$14.9 \quad 791$

$\begin{array}{llll}8 & 1.4 .8 & 79 & 1 \\ 8 & 14.8 & 79 & 1 \\ 7 & 14.6 & 79 & 1 \\ 5 & 3.4 .5 & 79 & 1 \\ 4 & 1.4 .8 & 77 & 1 .\end{array}$

12 15.0 781

14.5793 
page 5

$261.261 \times 2956.0 \quad 80 \quad 16.0 \quad 23 / 4 / 80 \quad 1855$

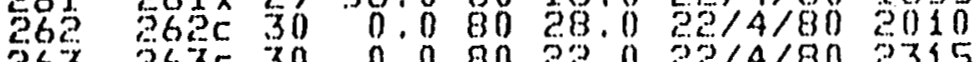

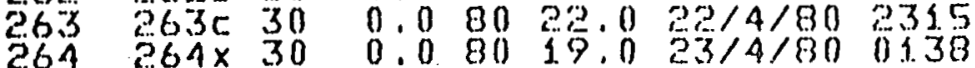

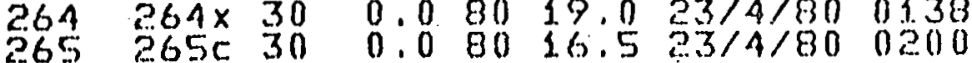

$266 \quad 266 \times 30 \quad 0.0 \quad 8013.5 \quad 23 / 4 / 80 \quad 0327$

267567030 0.0 $8010.523 / 4 / 800400$

268 268h 30 39.3 80 $10.123 / 4 / 8001010$

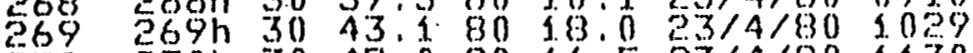

271 271h $3050.880 \quad 5.223 / 4 / 801230$

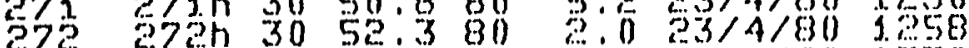

27.3 27343056.180 0.2 $23 / 4 / 80 \quad 1330$

274 274h 31 3.5 $7956.0 \quad 23 / 4 / 801425$

275 275h 31 5.2. $7950.023 / 4 / 801450$

276 276h $31.6 .57941 .5 \quad 23 / 4 / 80 \quad 1528$

279 377h 31 8.6 $7946.0 \quad 23 / 4 / 801600$

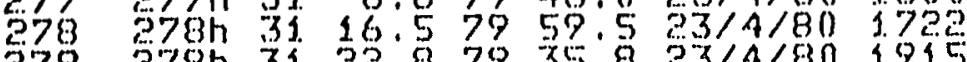

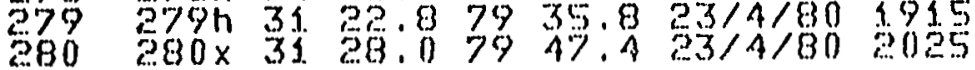

$281 \quad 281 \times 3122.47948 .423 / 4 / 802100$

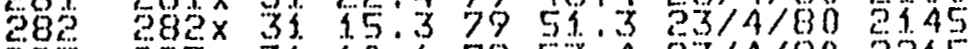

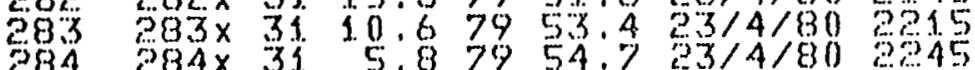
285 285x 31 1.4 $7959.35 / 4 / 80 \quad 535$

$2862866 \times 30 \quad 57.2795 \% .0 \quad 23 / 4 / 802345$ $28 \% \quad 387 \times 3053.580 \quad 3,324 / 4 / 800015$ $388388 \times 30 \quad 39.480 \quad 3.0 \quad 34 / 4 / 800045$ $599289 \times 3045.780 \quad 4.454 / 4 / 800115$ $290 \quad 290 \times 30 \quad 42.6 \quad 80 \quad 5.824 / 4 / 800145$

$291291 \times 30 \quad 39.580 \quad 7.124 / 4 / 80 \quad 0215$

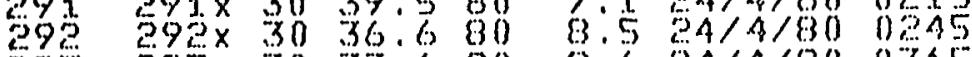
$293 \quad 293 \times 30 \quad 33.6 \quad 80 \quad 9.6 \quad 24 / 4 / 80 \quad 0315$

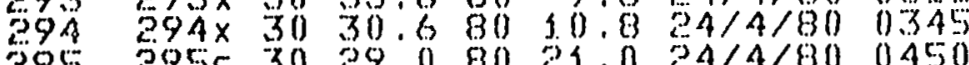

\section{9}

$296 \quad 2960 \quad 30 \quad 27.0 \quad 80 \quad 16.0 \quad 24 / 4 / 80 \quad 0640$ 297 29\% 30 26.0 80 13.0 24/4/80 0820

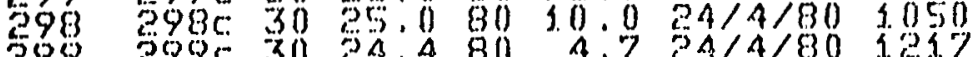

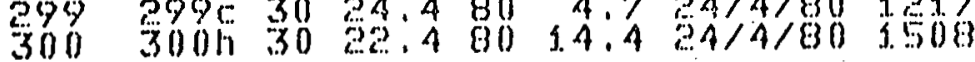

$301301 \times 3 n$ 19.0 80 1.4.0 $31 / 1.801530$ 303

304

305

306

$30 \%$

308

31.0

3.1.

312

$31 \%$

$31:$

316

$31 \%$

348

3.35

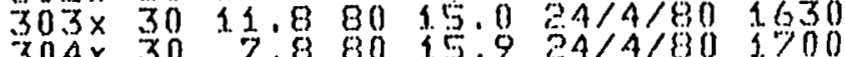

$3054 \quad 30$ 5.0 80 16.3 $24 / 4 / 80$ 17:21.

$30 \% 129 \quad 57.380 \quad 25.124 / 4 / 80 \quad 1830$

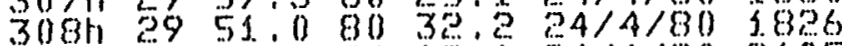

$309 h 29 \quad 3 \% .080$ 19.1 $24 / 4 / 80$ :10.5

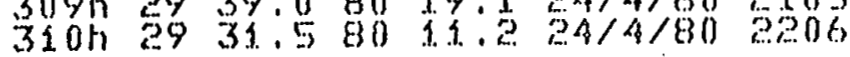

311h 28 28, 80 6.6 24/4/80 2048

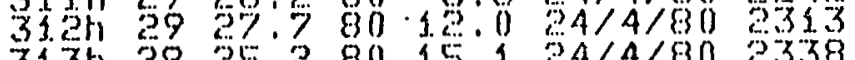

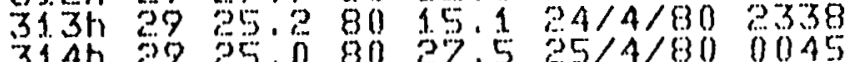

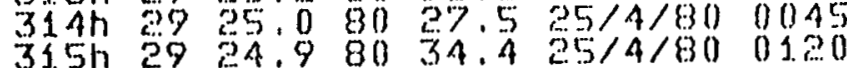

35.6h $29 \quad 34.9 \quad 8027.7 \quad 25 / 4 / 800030$

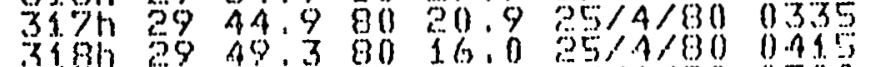

319420 4.2. 80 183.4 $5 / 4 / 800500$

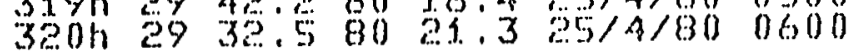

270 2.70h $30 \quad 47.0 \quad 80$ 11.5 $23 / 4 / 80$ 1.130 301 $302 \times 30$ 16:0 80 1.4. $24 / 4 / 80$ 1600

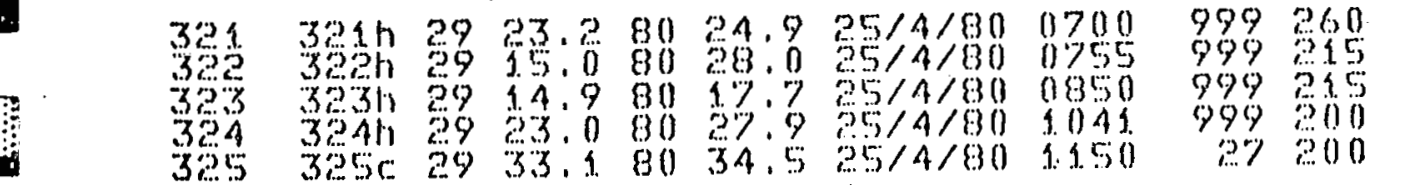

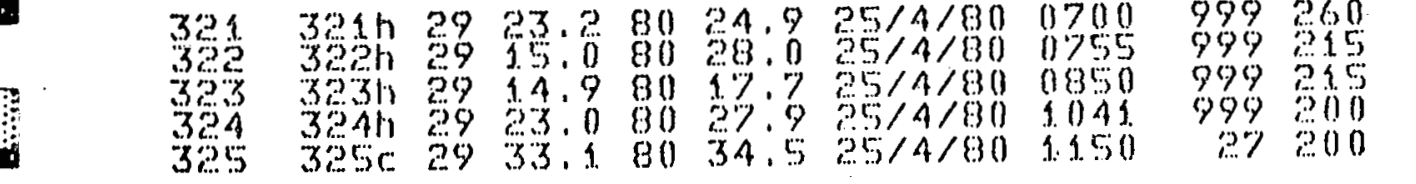

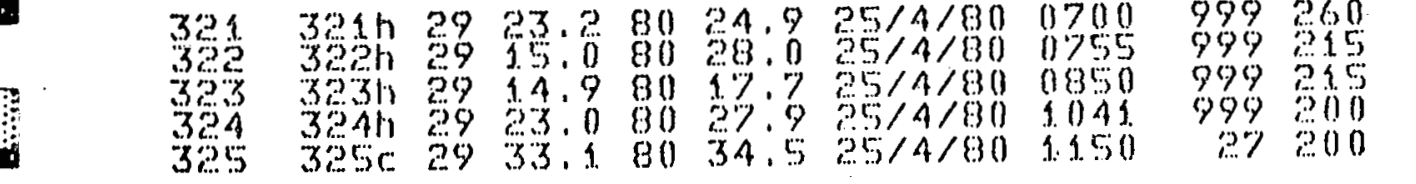

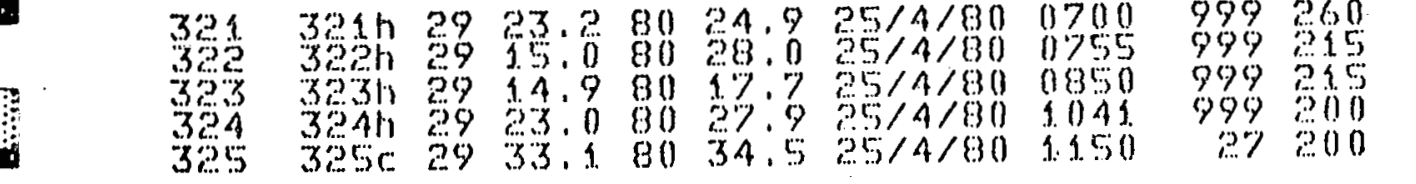

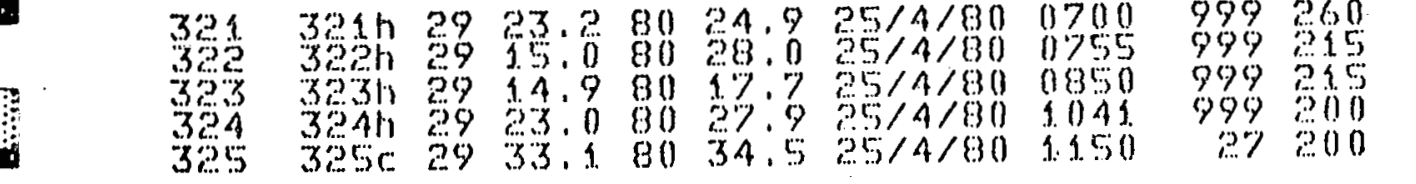

$\begin{array}{rrrr}72 & 0 & 2 & 205 \\ 41 & 80 & 2 & 170 \\ 48 & 190 & 3 & 190 \\ 51 & 190 & 3 & 205 \\ 67 & 190 & 3 & 205\end{array}$

$\begin{array}{rrrr}7 & 14.5 & 75 & 1 \\ 8 & 14.6 & 71 & 1 \\ 17 & 1.6 & 70 & 1 \\ 12 & 14.6 & 70 & 1 \\ 18 & 14.8 & 70 & 1\end{array}$

1841.90

2859595

999695

3 2 255

4 2⿺辶

4255

20
15
17

18

13.8

701

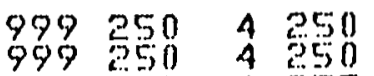

999250425

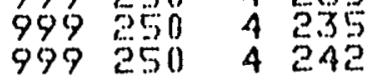

4240

999235

999250

999205

4215

4205

58395

4195

$\begin{array}{ll}60 & 195 \\ 74 & 205 \\ 58 & 220 \\ 63 & 200 \\ 68 & 20\end{array}$

4195

$\begin{array}{ll}65 & 295 \\ 55 & 205 \\ 67 & 250 \\ 63 & 205\end{array}$

63 2.5

$59 \quad 255$

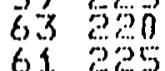

6)

40 Pि:

4225

4 ?

4205

14
14
15
15
15
17
16
17
26
18

14.0

3.3.8

$1 \frac{3}{3} \cdot 8$

73.1

1.3 .7740

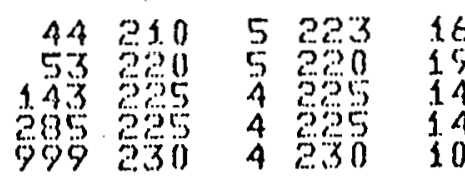

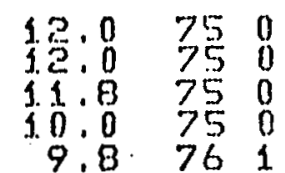

9.0761

$9.0 \quad 76.1$

8.2 731

$8.4 \quad 7351$

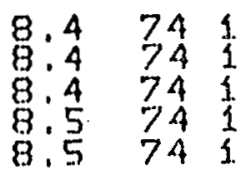

8.5 741

$8.5 \quad 741$.

$8.5 \quad 7.31$

$8.4 \quad 7 \% 1$.

420024

$\begin{array}{lll}4 & 250 & 34 \\ 5 & 250 & 23\end{array}$

8.5721

$8 \cdot 5 \quad 75$

$8.5 \quad 7 \% 1$

8.57

9.2691

9.469

$10.4 \quad 72 \quad 1$

1.0.5 $9: 040$

$68200 \quad 4200 \quad 14$

72000

4158

14

60300

890800

43.5

14

$8.8 \quad 7 \% 0$

$8.8 \quad 70$

$9.0,770$

$\begin{array}{lll}9.8 & 77 & 0 \\ 9.0 & 77 & 0\end{array}$

$306 \times 30 \quad 3.8 \quad 80 \quad 17.7 \quad 24 / 4 / 80 \quad 3.731$

802000

4 ... 150

8.8770

9991.45

4198

16

$9.0 \quad 750$

999380

4.500

16

999235

4335

15

9.8

9.8

743

$10: 3 \quad 741$

998265

406

$1 \frac{15}{15}$

999
999
965

4265

909265

of 26.5

10

90926

420.6

14

3. 0:

1. 0.4

5. 0.4

741

999200

s $2(0)$

12

999

999250

4 340

$99 \% 250$

3 향

12

1..

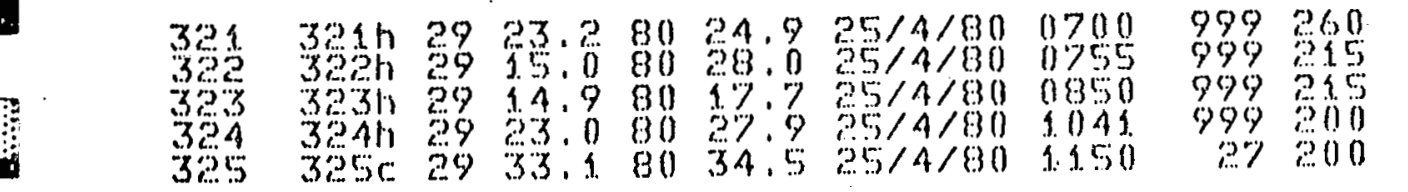


page 6

\begin{tabular}{|c|c|c|c|c|c|c|c|c|c|c|c|c|c|c|}
\hline $\begin{array}{l}326 \\
32.7 \\
328 \\
329 \\
330\end{array}$ & $\begin{array}{l}329 c \\
327 c \\
328 x \\
329 c \\
330 x\end{array}$ & $\begin{array}{l}29 \\
29 \\
29 \\
29 \\
29\end{array}$ & $\begin{array}{l}32.8 \\
33.0 \\
33.1 \\
33.1 \\
33.8\end{array}$ & $\begin{array}{l}80 \\
80 \\
80 \\
80 \\
80\end{array}$ & $\begin{array}{l}25 \cdot 1 \\
19.6 \\
1.5 .3 \\
8.8 \\
2.4\end{array}$ & $\begin{array}{l}25 / 4 / 80 \\
25 / 4 / 80 \\
25 / 4 / 80 \\
25 / 4 / 80 \\
25 / 4 / 80\end{array}$ & $\begin{array}{l}1420 \\
1553 \\
171.8 \\
1753 \\
1933\end{array}$ & $\begin{array}{r}36 \\
79 \\
73 \\
69 \\
109\end{array}$ & $\begin{array}{l}1.65 \\
140 \\
130 \\
150 \\
160\end{array}$ & $\begin{array}{l}\frac{3}{3} \\
\frac{3}{3} \\
3\end{array}$ & $\begin{array}{l}1.20 \\
130 \\
133 \\
151 \\
1.60\end{array}$ & $\begin{array}{l}6 \\
10 \\
10 \\
15 \\
13\end{array}$ & $\begin{array}{l}13 \cdot 4 \\
12 \cdot 8 \\
12.8 \\
12.8 \\
13.0\end{array}$ & $\begin{array}{l}74 \\
77 \\
77 \\
76 \\
76\end{array}$ \\
\hline $\begin{array}{l}331 \\
332 \\
333 \\
334 \\
3 \times 2\end{array}$ & $\begin{array}{l}331 h \\
332 h \\
333 h \\
334 h \\
335 h\end{array}$ & $\begin{array}{l}29 \\
29 \\
30 \\
30 \\
30\end{array}$ & $\begin{array}{r}46.0 \\
54 \cdot 2 \\
4.8 \\
1.2 \\
14.3 \\
14.3\end{array}$ & $\begin{array}{l}80 \\
80 \\
80 \\
80 \\
80\end{array}$ & $\begin{array}{l}10.0 \\
18.5 \\
15.4 \\
15.1 \\
21.6\end{array}$ & $\begin{array}{l}25 / 4 / 80 \\
25 / 4 / 80 \\
25 / 4 / 80 \\
25 / 4 / 80 \\
25 / 4 / 80\end{array}$ & 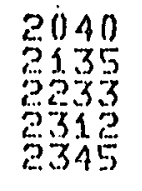 & $\begin{array}{l}999 \\
999 \\
999 \\
999 \\
990\end{array}$ & $\begin{array}{l}1.70 \\
1.65 \\
1.80 \\
180 \\
1.80\end{array}$ & $\begin{array}{l}3 \\
3 \\
3 \\
3\end{array}$ & $\begin{array}{l}9.70 \\
165 \\
180 \\
180\end{array}$ & $\begin{array}{l}18 \\
16 \\
15 \\
15 \\
15\end{array}$ & $\begin{array}{l}13.0 \\
13.0 \\
13.0 \\
13.0 \\
13.0\end{array}$ & $\begin{array}{l}76 \\
75 \\
74 \\
74 \\
74\end{array}$ \\
\hline $\begin{array}{l}336 \\
337 \\
338 \\
339\end{array}$ & $\begin{array}{l}336 c \\
337 c \\
338 x \\
339 c \\
340 c\end{array}$ & $\begin{array}{l}30 \\
30 \\
30 \\
30 \\
30\end{array}$ & $\begin{array}{l}16.8 \\
16 \cdot 6 \\
15 \cdot \frac{5}{5} \\
15.0 \\
15.0\end{array}$ & $\begin{array}{l}80 \\
80 \\
80 \\
80 \\
80\end{array}$ & $\begin{array}{r}30.4 \\
23.3 \\
3.6 \cdot 6 \\
14 \cdot 6 \\
5.8\end{array}$ & $\begin{array}{l}26 / 4 / 80 \\
26 / 4 / 80 \\
26 / 4 / 80 \\
26 / 4 / 80 \\
26 / 4 / 80\end{array}$ & $\begin{array}{l}0.30 \\
0.250 \\
0551 \\
0522 \\
0800\end{array}$ & $\begin{array}{r}37 \\
40 \\
54 \\
55 \\
310\end{array}$ & $\begin{array}{l}3.80 \\
3.80 \\
165 \\
3.85 \\
195\end{array}$ & $\begin{array}{l}3 \\
3 \\
3 \\
3 \\
3\end{array}$ & $\begin{array}{l}195 \\
185 \\
1.73 \\
190 \\
195\end{array}$ & $\begin{array}{l}19 \\
16 \\
16 \\
16 \\
14\end{array}$ & $\begin{array}{l}1.2 \cdot 8 \\
3.2 \cdot 5 \\
1.2 \\
13: 6 \\
1.3 \cdot 8\end{array}$ & $\begin{array}{l}74 \\
73 \\
73 \\
73 \\
76\end{array}$ \\
\hline $\begin{array}{r}342 \\
343 \\
344\end{array}$ & $\begin{array}{l}341 h \\
342 h \\
343 h \\
344 c \\
345 c\end{array}$ & $\begin{array}{l}30 \\
30 \\
30 \\
30 \\
30\end{array}$ & $\begin{array}{l}18.1 \\
3.3 \\
8.2 \\
6.0 \\
6.2\end{array}$ & $\begin{array}{l}80 \\
80 \\
80 \\
80 \\
80\end{array}$ & $\begin{array}{r}7.4 \\
1.6 \cdot 6 \\
2.7 \cdot 0 \\
3.9 \\
2.9 \\
2.8\end{array}$ & $\begin{array}{l}26 / 4 / 80 \\
36 / 4 / 80 \\
26 / 4 / 80 \\
26 / 4 / 80 \\
26 / 4 / 80\end{array}$ & $\begin{array}{l}1.000 \\
1100 \\
1.1000 \\
13000 \\
1438\end{array}$ & $\begin{array}{r}999 \\
999 \\
999 \\
40 \\
40\end{array}$ & $\begin{array}{l}1.90 \\
1.75 \\
175 \\
175 \\
175\end{array}$ & $\begin{array}{l}3 \\
3 \\
3 \\
3\end{array}$ & $\begin{array}{l}190 \\
175 \\
175 \\
175 \\
165\end{array}$ & $\begin{array}{l}18 \\
17 \\
17 \\
17 \\
16\end{array}$ & $\begin{array}{l}13.8 \\
13: 9 \\
13.9 \\
14.0 \\
14.2\end{array}$ & $\begin{array}{l}76 \\
76 \\
76 \\
78 \\
79\end{array}$ \\
\hline 8 & $\begin{array}{l}346 x \\
347 c \\
348 x \\
3496 \\
350 x\end{array}$ & $\begin{array}{l}30 \\
30 \\
30 \\
30 \\
30 \\
\times 0\end{array}$ & $\begin{array}{l}5.9 \\
5.8 \\
5.5 \\
5.4 \\
5.1\end{array}$ & $\begin{array}{l}80 \\
80 \\
80 \\
80 \\
80\end{array}$ & $\begin{array}{l}27.3 \\
24.9 \\
20.3 \\
14.6 \\
10.3\end{array}$ & $\begin{array}{l}26 / 4 / 80 \\
26 / 4 / 80 \\
26 / 4 / 80 \\
26 / 4 / 80 \\
26 / 4 / 80\end{array}$ & $\begin{array}{l}5945 \\
1900 \\
1914 \\
1940 \\
3133\end{array}$ & $\begin{array}{r}42 \\
43 \\
49 \\
55 \\
200\end{array}$ & $\begin{array}{l}160 \\
160 \\
2700 \\
2700 \\
270\end{array}$ & $\begin{array}{l}3 \\
3 \\
3 \\
7\end{array}$ & $\begin{array}{l}290 \\
300 \\
105\end{array}$ & $\begin{array}{l}14 \\
14 \\
14 \\
16 \\
10\end{array}$ & $\begin{array}{l}13.0 \\
3,3.0 \\
14.0 \\
3.4 .8 \\
13.4\end{array}$ & $\begin{array}{l}79 \\
78 \\
79 \\
76 \\
75\end{array}$ \\
\hline & 3 & 30 & 5.0 & 80 & 6.8 & $36 / 1 / 80$ & 2.15\}. & 350 & 520 & 3 & 135 & 43 & 12.7 & 75 \\
\hline
\end{tabular}




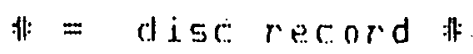

gtation =: ascigined station number

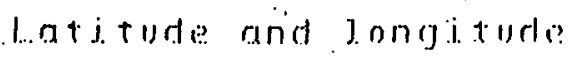

bate and time in enstern standred time

7. = water depth in meters

WUI) = wrove direction

SS :- SEA GTATF

WND = wind direction

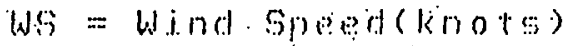

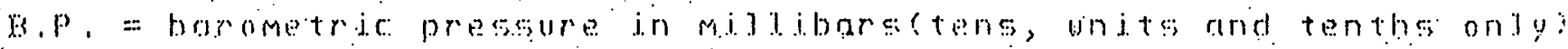

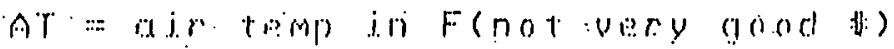

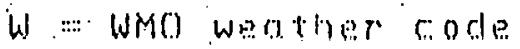

wgos indirate no data 
$13.3151 .68047 .510 / 4 / 802200$

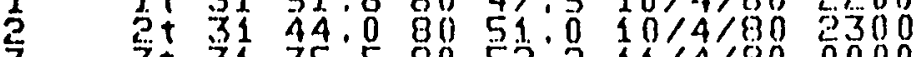
$3+3135: 580$ 5:2.2 $11 / 4 / 800000$ $4+3127.28053 .6 \quad 11 / 4 / 80.0300$ $5+3119.28054 .711 / 4 / 800200$

6 $6 t 3110.58055 .511 / 4 / 800300$ $7 \quad 7+31$ 2.0 80 55.3 $11 / 4 / 800400$ $8+3052.68055 .411 / 4 / 800500$ $9+3043.78055 .851 / 4 / 800600$ $1030 t \quad 30.34 .3 .8056 .2 .11 / 4 / 800700$

11 11. $3026.88055 .011 / 4 / 800800$ 1. 12 . $3030.58045 .111 / 4 / 800900$ $133.3+3034.88036 .031 / 4 / 801000$ $1434+3039.06027 .011 / 4 / 801100$ 15 1.5t $3045.0 \quad 80 \quad 19.013 / 4 / 801.200$

16 $16 \mathrm{t} 3041.08021 .211 / 4 / 801300$ 1.750130 .35 .0 80 25:5 $11 / 4 / 801400$ $1838+3042.78016 .511 / 4 / 801500$

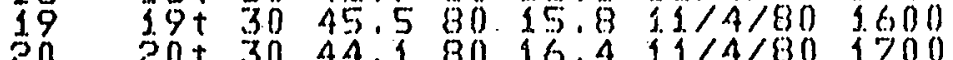

2. $21+3035.0 .8039 .53 .1 / 4 / 8030300$

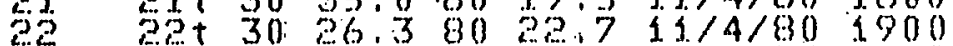
2.3 2.5 $23+30.3 \% \cdot 180$ 3. $33 / 4 / 802000$ $24+3013.780 .28 .0 .11 / 4 / 802100$ $25+29.59 .58034 .213 / 4 / 802200$

$262602950.88034 .1 \quad 11 / 4 / 802300$ $27.27+2942.08037 .512 / 4 / 800000$ $2839+2933.08041 .013 / 4 / 800100$ $2929+2924.08044 .013 / 4 / 800000$ $30 \quad 30+29 \quad 15.48046 .012 / 4 / 800300$

$\begin{array}{llllllll}31 & 1 \mathrm{c} & 29 & 8.2 & 80 & 48.5 & 1.2 / 4 / 80 & 0357\end{array}$

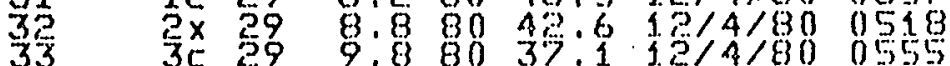

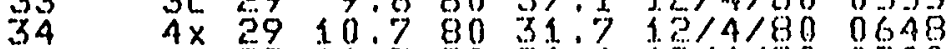

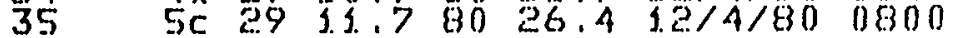

$36 \quad 6 \times 29 \quad 13.0 \quad 8020.8 \quad 12 / 4 / 80 \quad 0905$ 37

38

40

4

42

44

45

48

48

49
50

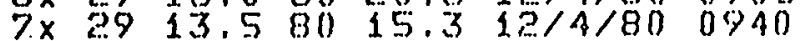

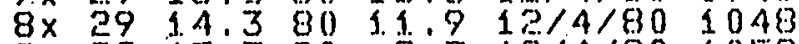

$9 \times 293.5 .380 \quad 9.7 \quad 12 / 4 / 801058$ $10 \times 2915.080 \quad 6.012 / 4 / 801200$

$3.1 \times 29 \$ 5.580$ 4. 0 1.2/4/80 3.256 $42 t 2919.280$ $43+2.29 .480$ $44+2931.580$ 3.9 12/4/80 1330 $3.9 \quad 12 / 4 / 80 \quad 3400$ $6.535 / 4 / 80 \quad 1500$ $45+2945.780$

\section{$7.512 / 4 / 801535$}

$46+2948.780 \quad 7.813 / 4 / 801630$ $47+2952.680$ 1. $5.732 / 4 / 803.700$

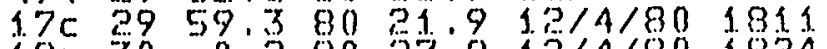
$38 \times 30 \quad 0.28007 .8 \quad 13 / 4 / 80 \quad 3024$

$51.20 \times 30 \quad 0.08039 .83 .2 / 4 / 8023.36$

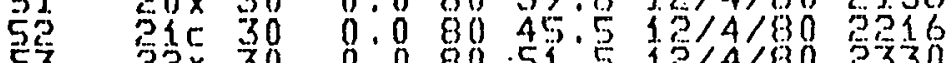

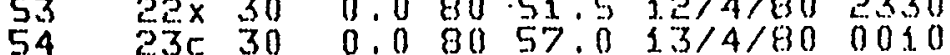
$55 \quad 24 t \quad 30$

$0.08120 .9 \quad 3.3 / 4 / 800331$.

$56 \quad 250 \quad 30 \quad 0.0 \quad 81 \quad 8.513 / 4 / 80 \quad 0205$

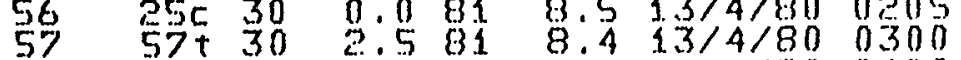
$58 \quad 58+30 \quad 12.381$ $59 \quad 54+30$ 25. 30 $7.9 \quad 13 / 4 / 800400$ 6.8 $13 / 4 / 80$ 0500 $60 \quad 60 t \quad 30$ 32.28 $815.513 / 4 / 800600$

61. 61+ $3042.083 .4 .43 .3 / 4 / 800 \% 00$

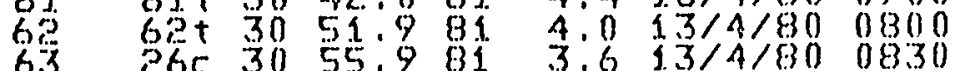
$64 \quad 57 \times 3055.0 \quad 8058.013 / 4 / 800955$ 65 286 $30 \quad 53.5$ 830 $50.53 .3 / 4 / 80$ 1.005 $\begin{array}{llllllll}11 & 225 & 2 & 225 & 10 & 14.2 & 66 & 0 \\ 13 & 213 & 2 & 213 & 12 & 14.8 & 64 & 0 \\ 15 & 213 & 2 & 213 & 12 & 14.6 & 65 & 0 \\ 15 & 213 & 2 & 213 & 12 & 14.9 & 65 & 0 \\ 15 & 213 & 2 & 213 & 12 & 14.9 & 64 & 0\end{array}$

$\begin{array}{llllllll}15 & 213 & 2 & 213 & 12 & 14.9 & 64 & 0 \\ 19 & 255 & 2 & 225 & 12 & 14.9 & 64 & 0 \\ 25 & 225 & 2 & 225 & 12 & 15.6 & 64 & 0 \\ 22 & 213 & 2 & 213 & 4 & 16.3 & 63 & 1 \\ 26 & 270 & 2 & 270 & 6 & 17.2 & 66 & 0\end{array}$

$26 \quad \therefore 83 \quad 2 \quad 283$

31 283 28283

33315

$40 \quad 31.5 \quad 2 \quad 345$

38 315 30 315

$\begin{array}{ll}39 & 45 \\ 40 & 45\end{array}$

$\begin{array}{llll}38 & 45 & 1 & 45 \\ 38 & 45 & 2 & 45 \\ 37 & 45 & 2 & 45\end{array}$

$35 \cdot 45$

$3.3 \quad 235$

293.35

26135

2.0. 135

21. 1.35

283.35

20135

33135

37 1. 35

$78 \quad 135$

$173 \quad 135$

$357 \quad 1.35$

$400 \quad 135$

$999 \quad 135$

$999 \quad 135$

9991.35

362160

1. 19960

45160

44960

41160

349.60

37300

:5 180

$24 \quad 180$

171801.80

9993.80

$24 \quad 180$

23380

24180

16 170

1. 8.60

31160

3450
2315

i. 45

1. 45

2. 135

23.35

a. 135

2135

$\begin{array}{ll}2 & 1 \\ 3 & 135 \\ 3 & 135\end{array}$

$3 \frac{1}{3} \quad 35$

31.35

$3 \frac{135}{3}$

$\begin{array}{ll}3 & 225 \\ 3 & 135\end{array}$

31.35

3 3. 35

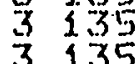

3135

3160

3360

41.60

4160

3160

3180

21.80

$\begin{array}{ll}3 & 1830 \\ 2 & 180\end{array}$

2180

3170

$\begin{array}{llll}6 & 18.2 & 68 & 0 \\ 5 & 1.8 .8 & 66 & 0 \\ 5 & 19.2 & 68 & 0 \\ 6 & 19.5 & 68 & 0 \\ 6 & 19.4 & 68 & 0\end{array}$

8 19.2 730

$\begin{array}{llll}3 & 17.7 & 77 & 1 \\ 5 & 17.7 & 75 & 1 .\end{array}$

$\begin{array}{llll}5 & 17.8 & 7.4 & 1 \\ 5 & 17.9 & 73 & 1 \\ 6 & 18.2 & 72 & 1 \\ 4 & 1.8 \cdot 5 & 72 & 1 \\ 4 & 1.8 .8 & 70 & 1\end{array}$

$\begin{array}{rlll}8 & 1.8 \cdot 4 & 70 & 1 \\ 8 & 1.8 \cdot 2 & 69 & 1 \\ 8 & 1.7 \cdot 6 & 69 & 1 \\ 10 & 1.7 \cdot 3 & 69 & 1 \\ 10 & 1.6 \cdot 6 & 69 & 1\end{array}$

1) 1.6 .6 .691

$\begin{array}{llll}13 & 16.5 & 70 & 1\end{array}$

$\begin{array}{llll}14 & 16.7 & 71 & 1\end{array}$

$18 \quad 17.5 \quad 735$

16 3.6.8 74

16017.0

$12 \quad 16.6 \quad 761$

130.4 .979

1. 14.4801

$15 \quad 14.2771$

$15 \quad 14.2 \quad 751$

$17 \quad 9.3 .9 \quad 734$

$\begin{array}{llll}17 & 13.9 & 72 & 1 \\ 15 & 13.9 & 71 & 1\end{array}$

$15 \quad 14.4 \quad 701$

$\begin{array}{llll}15 & 1.4 .4 & 70 & 1 \\ 1.0 & 14.8 & 70 & 9 \\ 5 & 1.4 .5 & 69 & 5 \\ 5 & 1.3 .9 & 69 & 5\end{array}$

833.4695

$8 \quad 3.5 \quad 6 \% 1$

8. 12.5 68 1

$8 \quad 12.5 \quad 681$

$643.3 \quad 681$

$\begin{array}{lllll}10 & 12.3 & 68 & 1\end{array}$

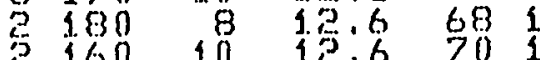

$\begin{array}{lllll}360 & 10 & 12.6 & 70 & 1\end{array}$
$5 \quad 18.0761$

$3017.6 \quad 741$

1.6 $17.0 \quad 751$ 
page 2

$66 \quad 29 \times 30 \quad 52.5 \quad 80 \quad 46.7 \quad 13 / 4 / 80 \quad 1059$

$6730 \mathrm{C} 3051.080041 .3 \quad 13 / 4 / 80 \quad 1.30$

$6831 \times 3050.0 \quad 80 \quad 35.5 \quad 13 / 4 / 801259$

$69 \quad 32 c \quad 3049.0 \quad 8030.013 / 4 / 801318$

$70 \quad 33 \times 30 \quad 47.5 \quad 8024.0 \quad 13 / 4 / 80 \quad 1441$

$7134 \mathrm{C} \quad 30 \quad 46.5 \quad 80 \quad 18.5 \quad 13 / 4 / 80 \quad 1526$

$72 \quad 35 \times 3045.56015 .813 / 4 / 80 \quad 1649$

$7437 x \quad 3045.0 \quad 8010.013 / 4 / 80184.4$

$75 \quad 38 c \quad 30 \quad 44.0 \quad 807 \% 25 / 4 / 830 \quad 1839$

$76 \quad 39 \times 30 \quad 44.280 \quad 4.3 \quad 13 / 4 / 80 \quad 1943$

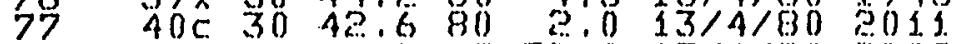

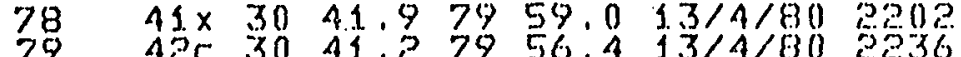

$8043 \times 3042.07951 .514 / 4 / 800100$

81. $81+3047.07948,514 / 4 / 800330$

8. 82 t $3053.57946 .8 \quad 14 / 4 / 800000$

83

834

85

86

838

89

90

91. $47 \times 31.29 .57941 .0 \quad 14 / 4 / 801.20 \%$

$92 \quad 48 \times 3130.07944 .0 \quad 14 / 4 / 90 \quad 1305$

9.3

94

96

97

99

100

1. 01

5. 0.2

104

105

106

108

309

1.1.

1.1.

1.14

1.15
$83+3059.57943 .3 .4 / 4 / 8300330$

$84+31.6 .27941 .014 / 4 / 800300$

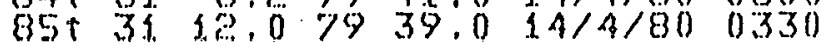

$86+31.18 .37936 .534 / 4 / 800400$

$87+31.24 .37934 .354 / 4 / 800430$

$44 C \quad 3127.3 \quad 7933.0 \quad 14 / 4 / 800449$

$45 \times 39.28 .27935 .7 \quad 14 / 4 / 8013646$

$46 \times 3159.37937 .7 \quad 94 / 4 / 80 \quad 0704$

$93+3130.57946 .5 \quad 34 / 4 / 803.400$

$94+31.5 .2795 \%, 014 / 4 / 801500$

$95+3138.77958 .13 .4 / 4 / 80$ 3.600

$96+31.19 .3 \quad 80 \quad 3.7 \quad 14 / 4 / 80 \quad 1700$

$97+315.580$ \%.4 $34 / 4 / 803800$

$98+3058.780 \quad 15.1 .4 / 4 / 80$ 1.900

$89+3052.0 .8020 .6,3 / 4 / 802000$ $100+3047.08025 .4 \quad 14 / 4 / 802100$

1.01t $3042.48030 .034 / 4 / 802200$ $102+3037.38034,534 / 4 / 805300$ $103+30 \quad 32.5 \quad 8039.5 \quad 3.5 / 4 / 800000$ $104 t 3027.58044 .015 / 4 / 800100$ j. $05+3053,0$ 60 $48: 0 \quad 35 / 4 / 800200$

$106+30.18 .280 \quad 53.0 \quad 15 / 4 / 80 \quad 0300$ $307+3013.4 .8059,25 / 4 / 800400$ $108+307.784 \quad 4.25 / 5 / 800500$ $3.09+30$ $1.10+30$

4.783.

$5 . \% 5 / 4 / 80$

2.481

$7.3 \quad 15 / 4 / 80$

0700

$250 \quad 30$

0.081

8. $815 / 4 / 810$

08304

$24 \times 30$

$236 \quad 30$

$22 \times 30$

0.08

0.080

$3.1 \quad 15 / 4 / 80$

23.C 30

$51.015 / 4 / 801256$ $\begin{array}{ll}28 & 160 \\ 31.60 & 9.60\end{array}$

29160

35 1.60

$42 \quad 160$

41. 1.60

$43 \quad 150$

46.150

49150

80160

198360

ग10 1.60

396160

390160

999160

999160

9993.60

999160

9993.60

$417 \quad 160$

31.360

$296 \quad 165$

391.70

$100 \quad 3.80$

683180

59091.80

990180

4250

48900

$38 \quad 230$

37 5.30

41230

37235

33240

999240

999
999
950

$999 \quad 250$

$24 \quad 580$

18 3.5

s. 6.90

21 270

39270

23570

26260

29260

$\begin{array}{ll}35 & 260 \\ 41 & 560\end{array}$

38260

$4 \hat{2} \div 0$

483265

$48 \quad 265$

68255 $\begin{array}{llllll}3 & 160 & 13 & 11.8 & 72 & 0 \\ 3 & 160 & 16 & 11.8 & 72 & 0 \\ 3 & 160 & 16 & 11.5 & 71 & 0 \\ 3 & 160 & 16 & 11.1 & 76 & 0 \\ 3 & 160 & 16 & 9.5 & 76 & 0\end{array}$

316016

$4150 \quad 16$

415016

415016

$\begin{array}{lll}4160 & 18 \\ 4 & 160 & 24\end{array}$

116024

460
4
160

416028

416026

416026

436026

416024

4 1. 60 24

4165

4170

4160

26

$\begin{array}{ll}5 & 180 \\ 5 & 280 \\ 5 & 270\end{array}$

4270

4 23.

30

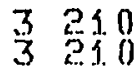

3230

3230

28
25
25
25

3230

4235

4240

4240

4250

4280

4
3
3 250

3270

2.: 27

2 要6

2560

$2 \pi 0$

15
1.5
20
1.8
16

2260

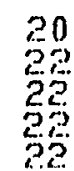

\section{?.?}

1.

is

1.2
1. 1.6

5.17

1. 18

1.20

1.2.

1.

123

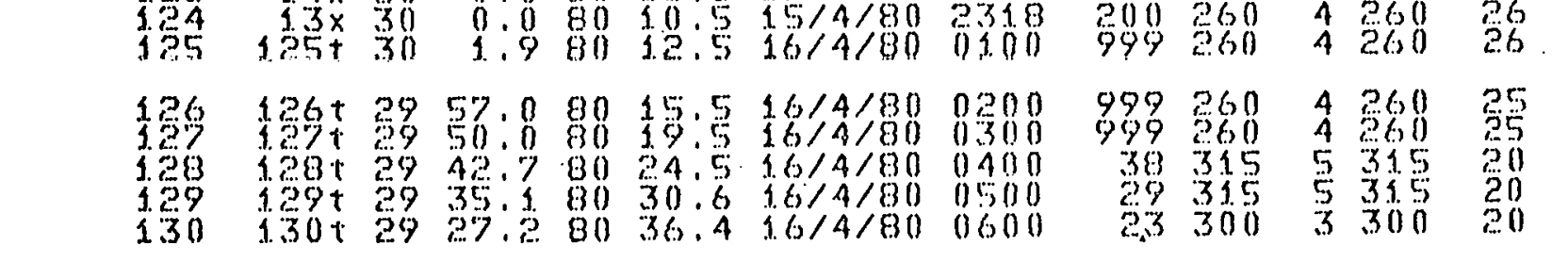

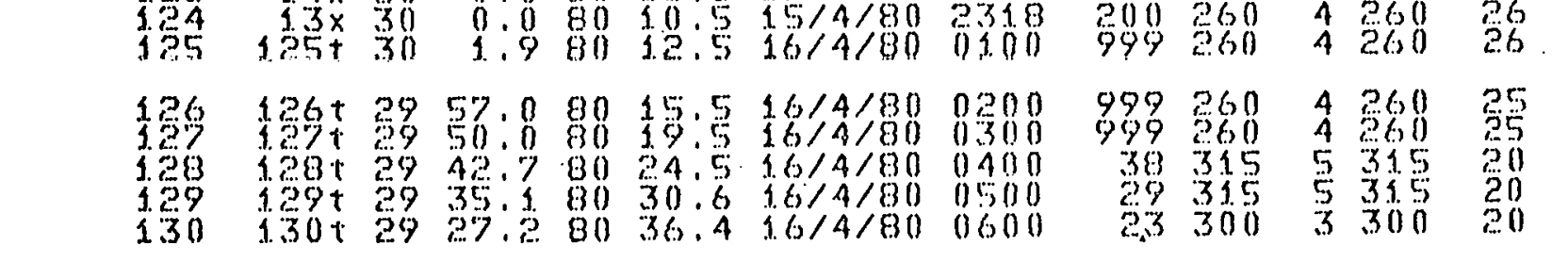

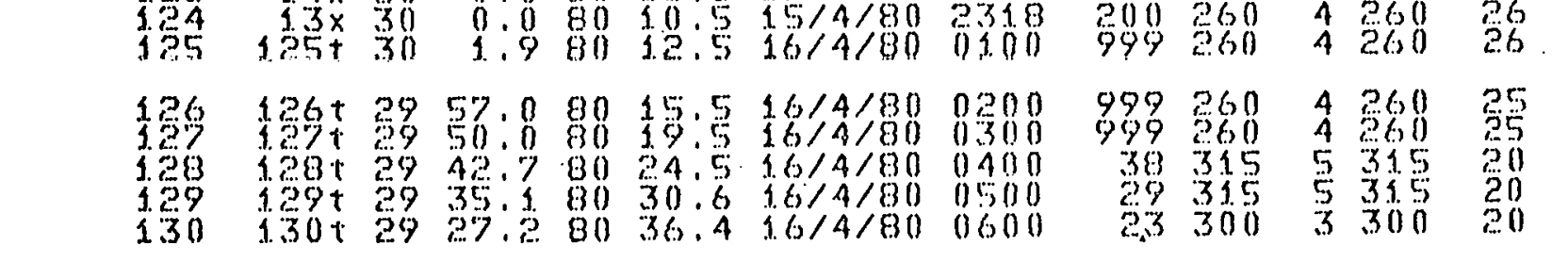

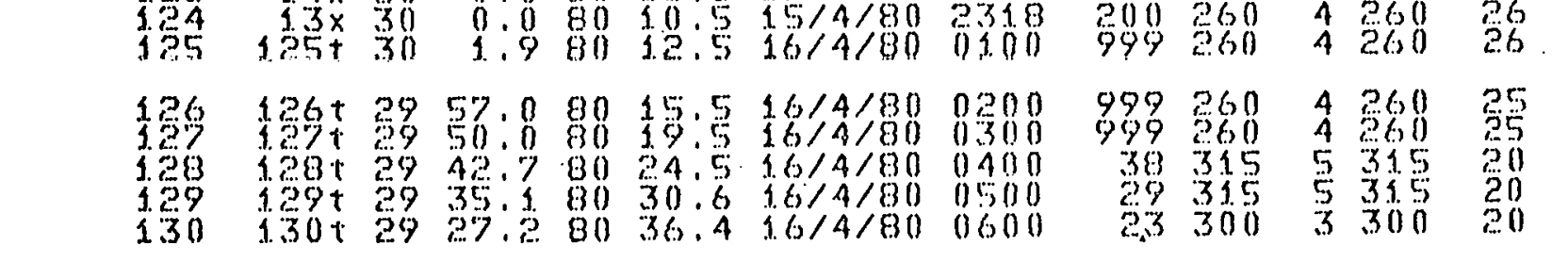

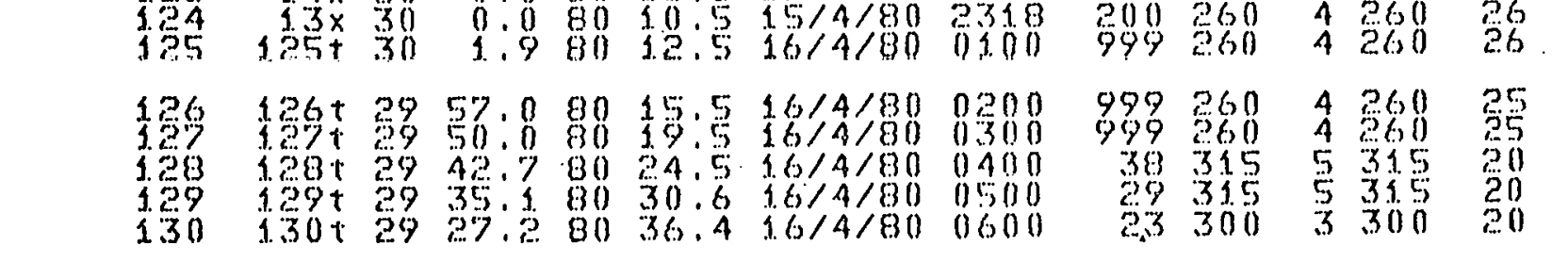

$20 \times 30$

$3.79+30$

1.90 30

$1.8 \times 30$

17e 30

$0.0 \quad 80 \quad 39.5 \quad 15 / 4 / 80 \quad 1445$

$0.08035: 5$ j.5/4/80 3507

$0.0 \quad 80 \quad 33.5 \quad 15 / 4 / 80 \quad 1535$

0.0 80 $27.735 / 4 / 80 \quad 3934$

$0.08022 .015 / 4 / 802001$

$1.6 \times 30$

$0.0 \quad 80 \quad 19.5 \quad 15 / 4 / 80 \quad 21.23$

$150 \quad 30$

$3.4 \times \quad 30$

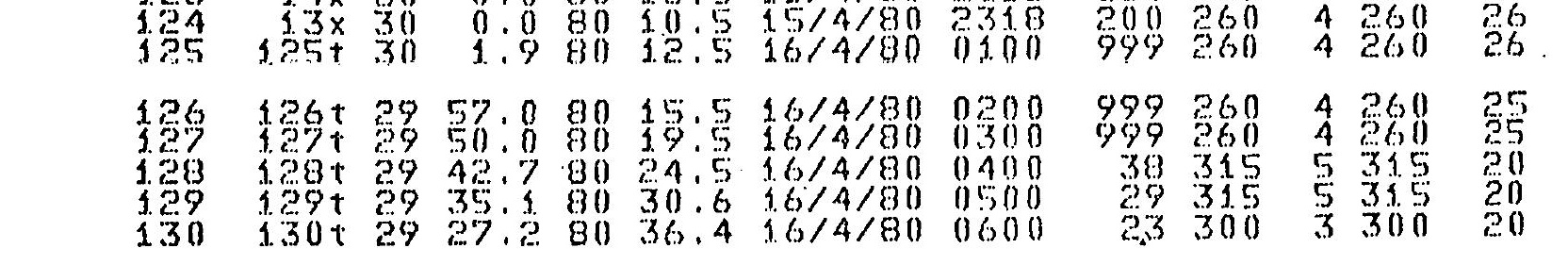

$0.0 \quad 80 \quad 16.5 \quad 15 / 4 / 80$

$0.080 \quad 33.5 \quad 15 / 4 / 80$

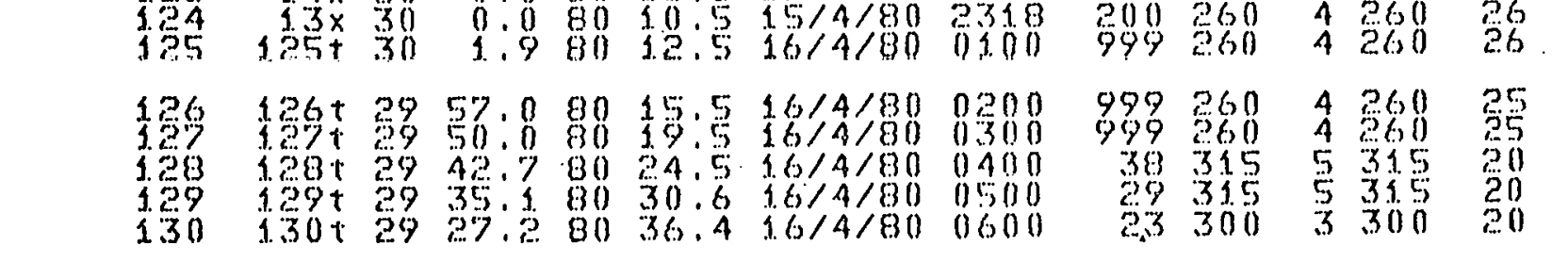

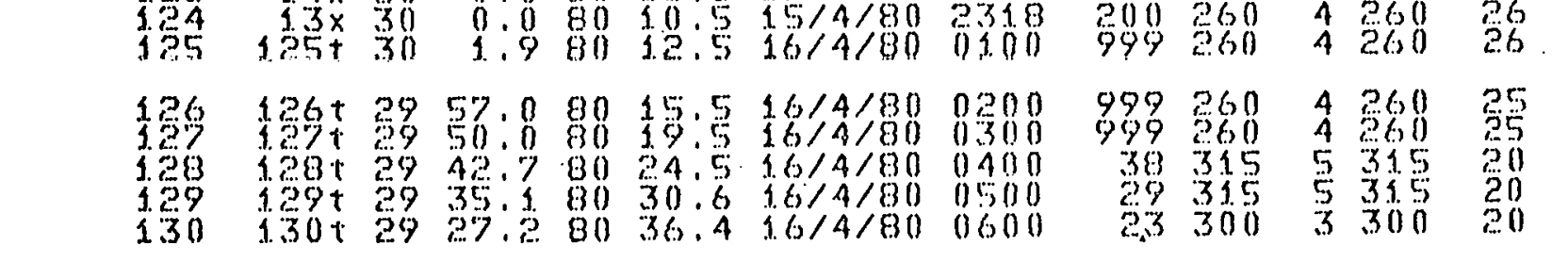

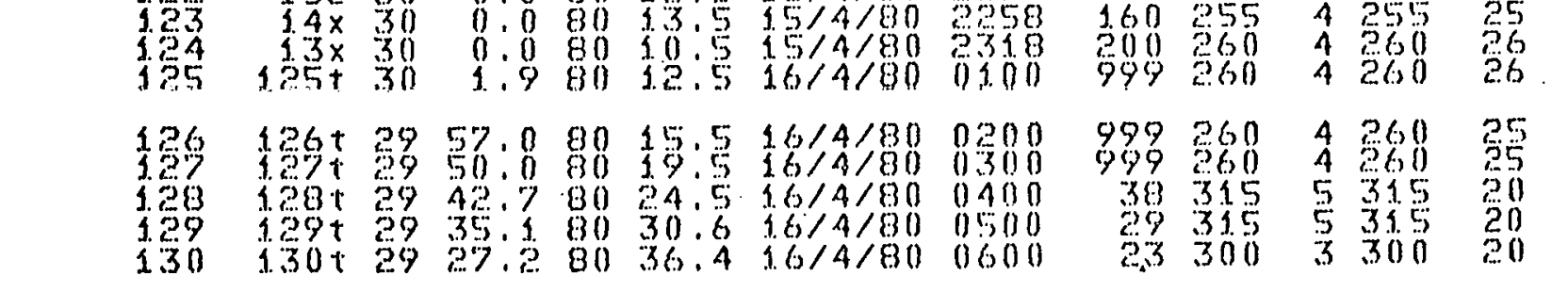

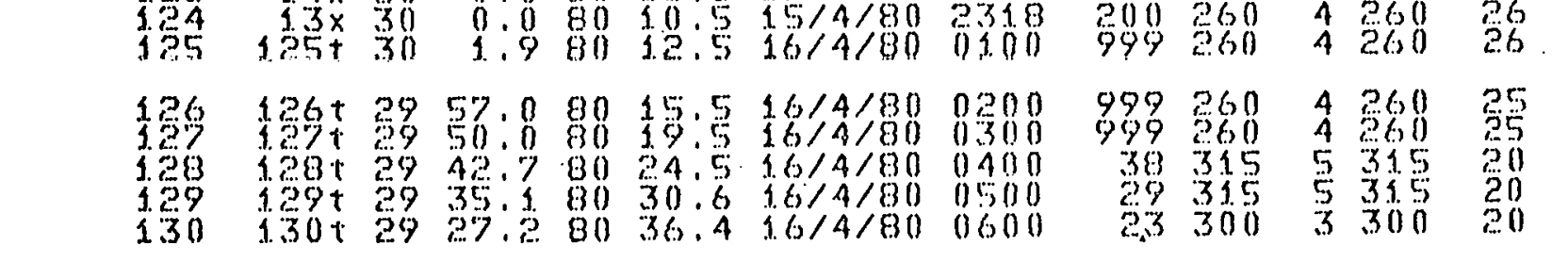

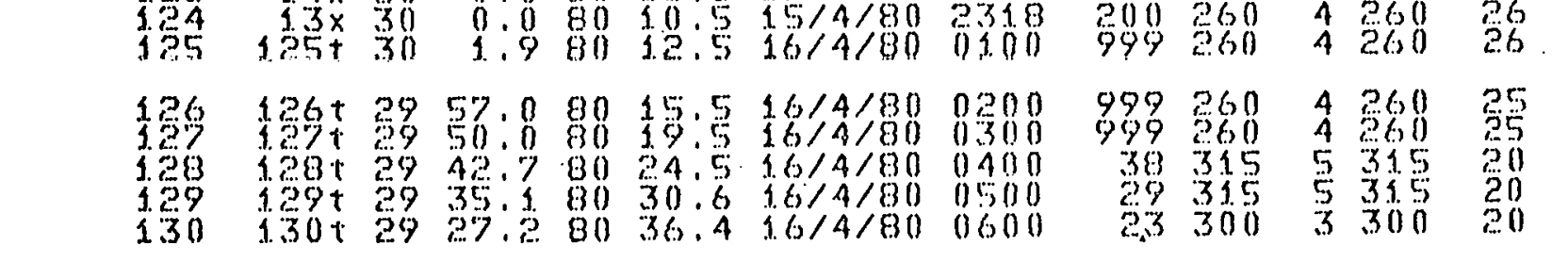

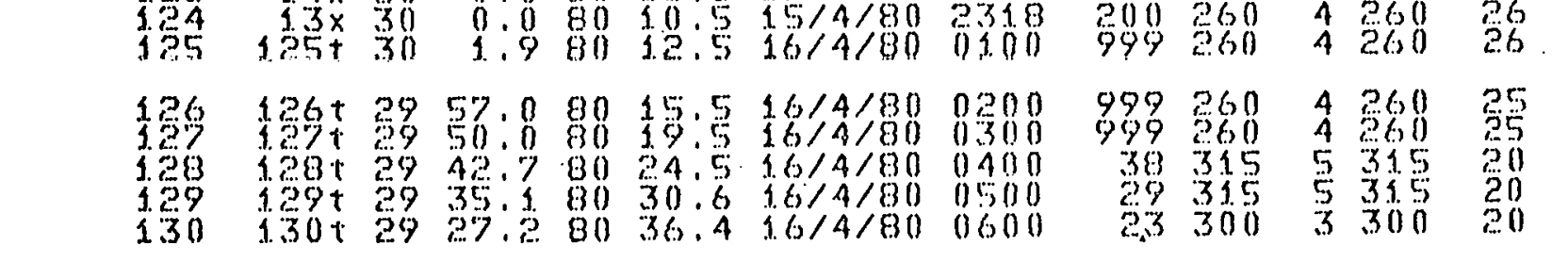

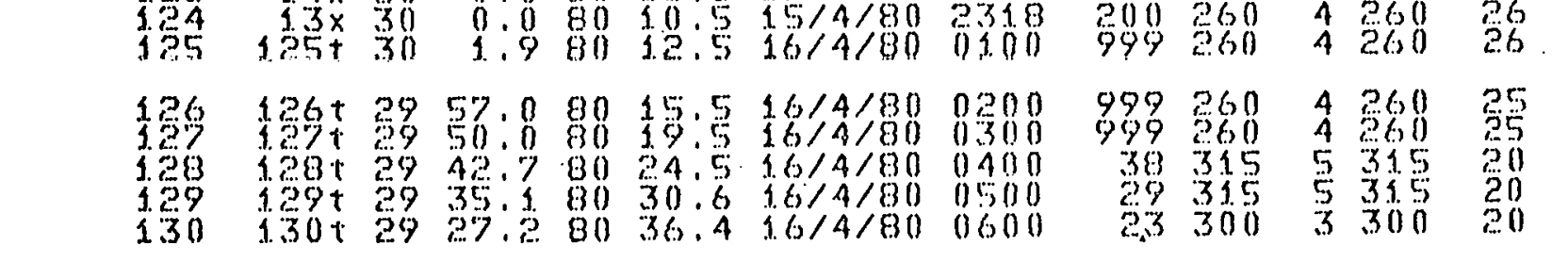

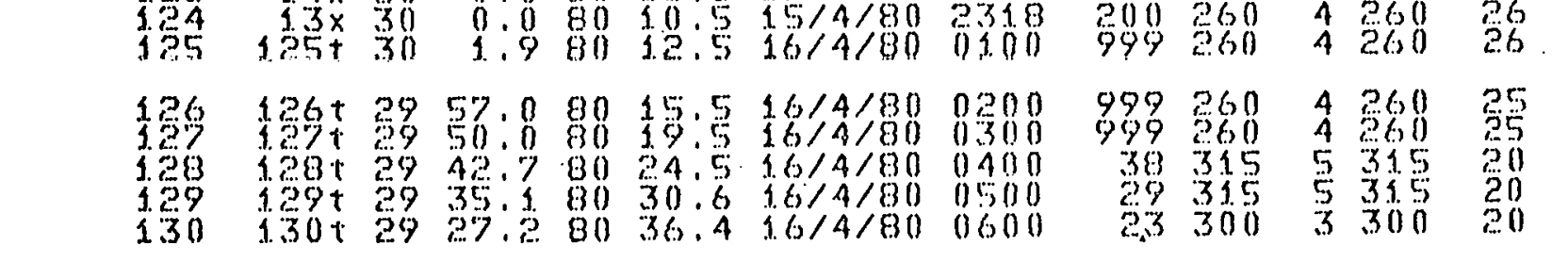

2560

2 260

3265

3265

4 近

4255

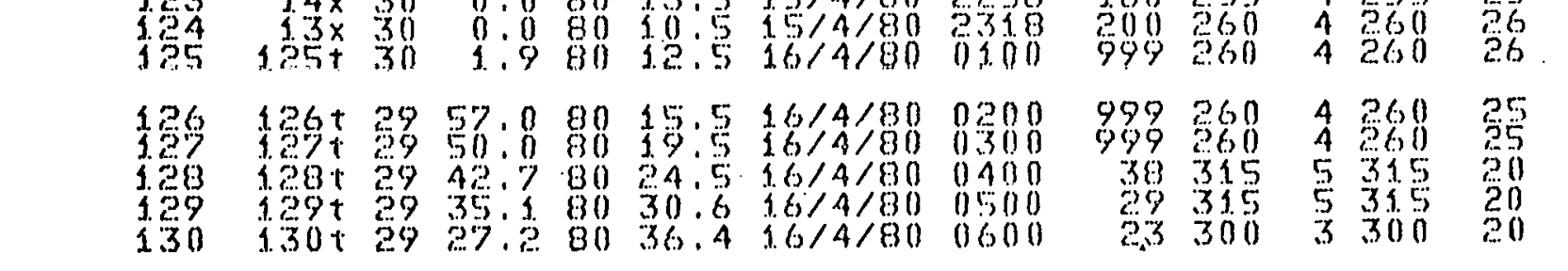

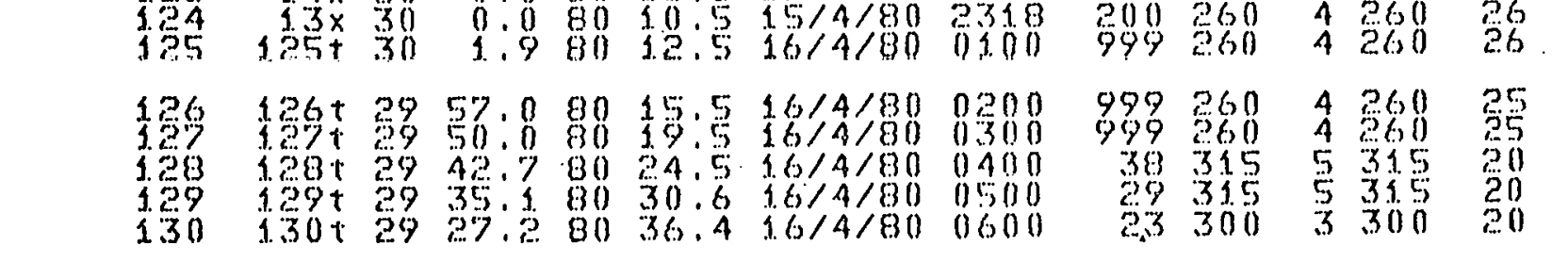

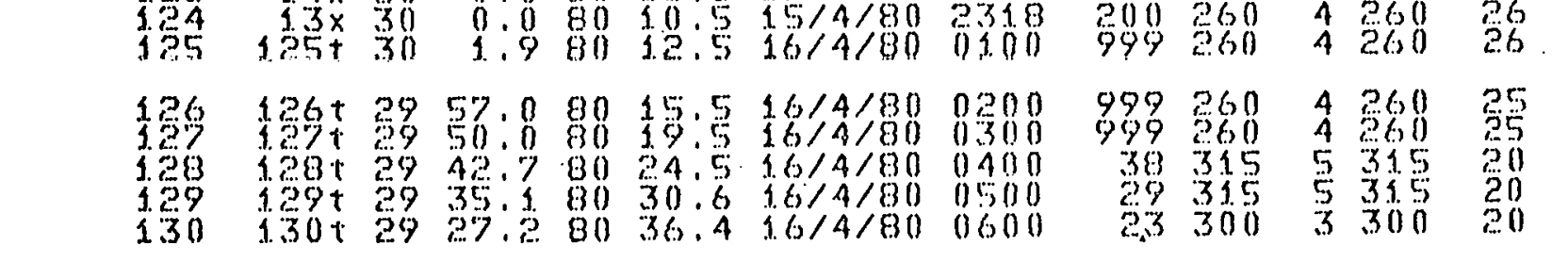

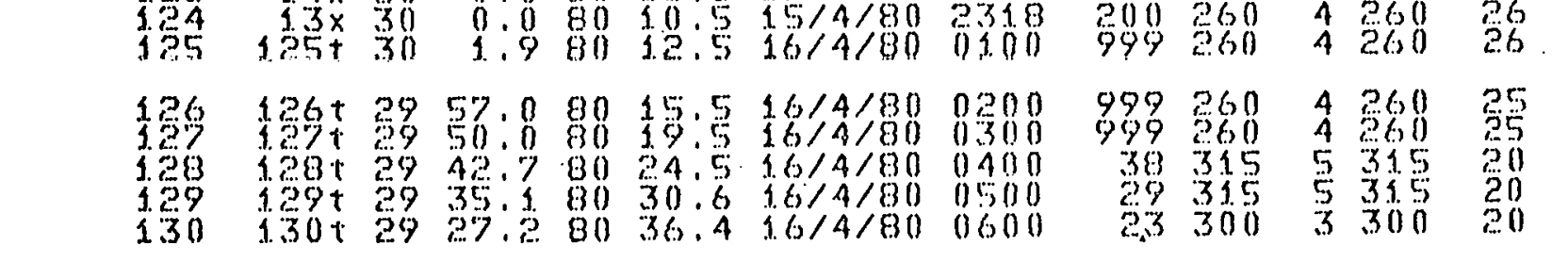

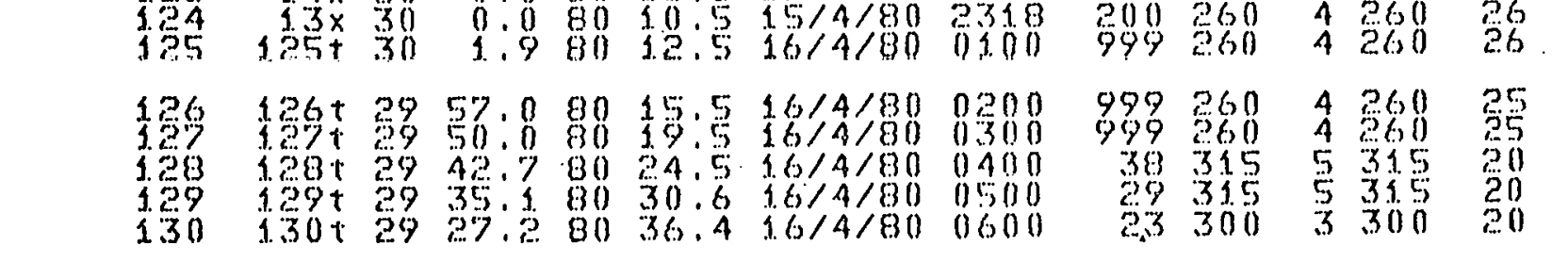

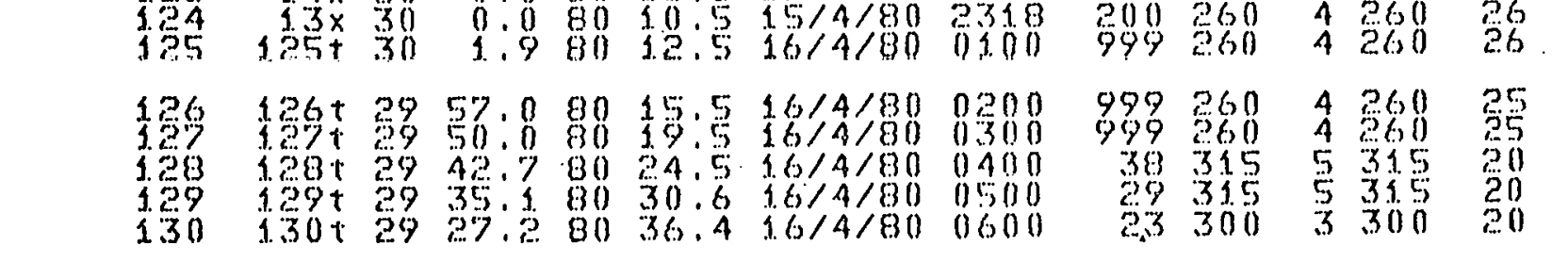

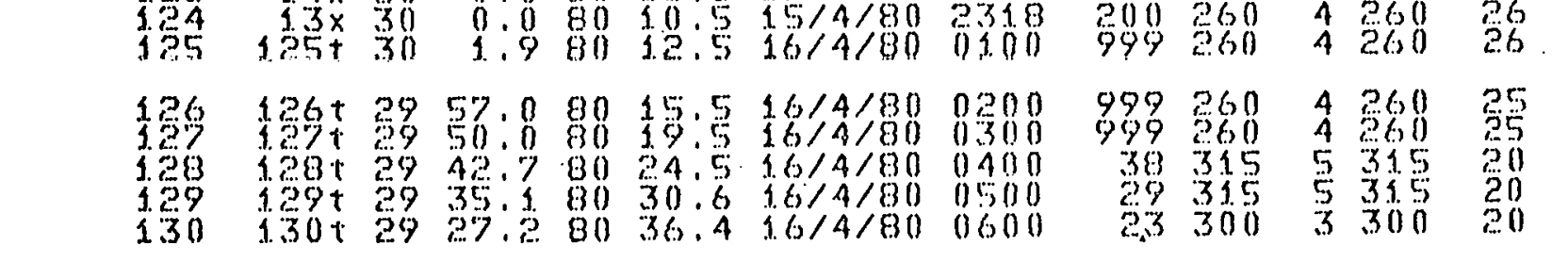

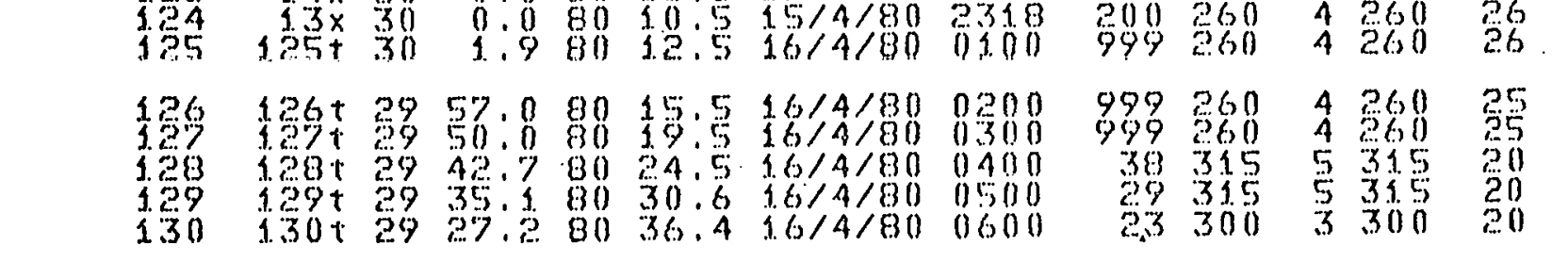

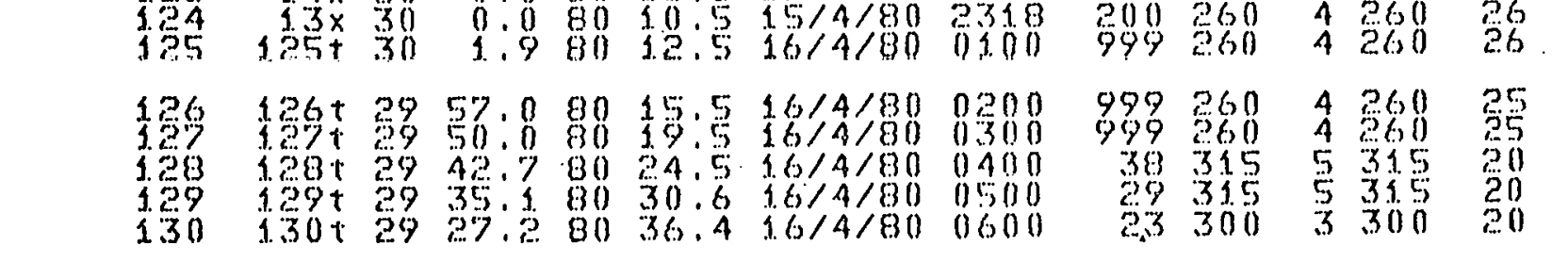

3. 0

10

1.8

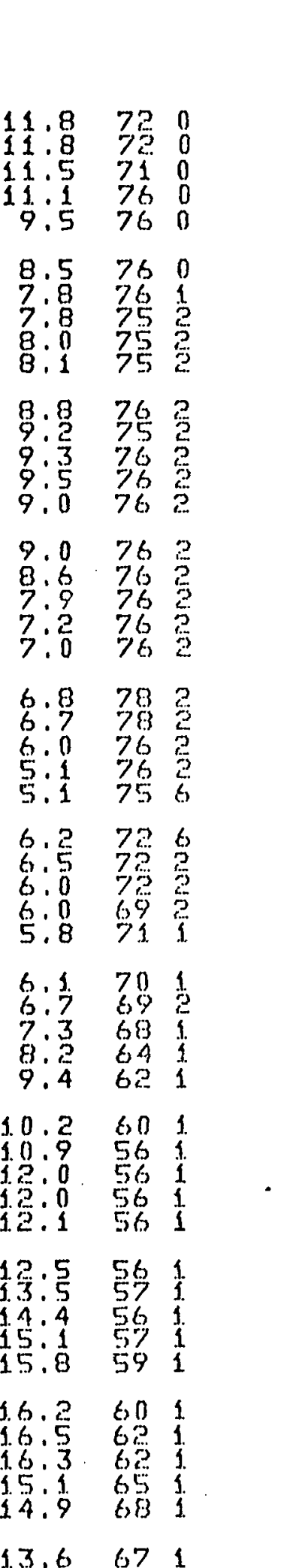

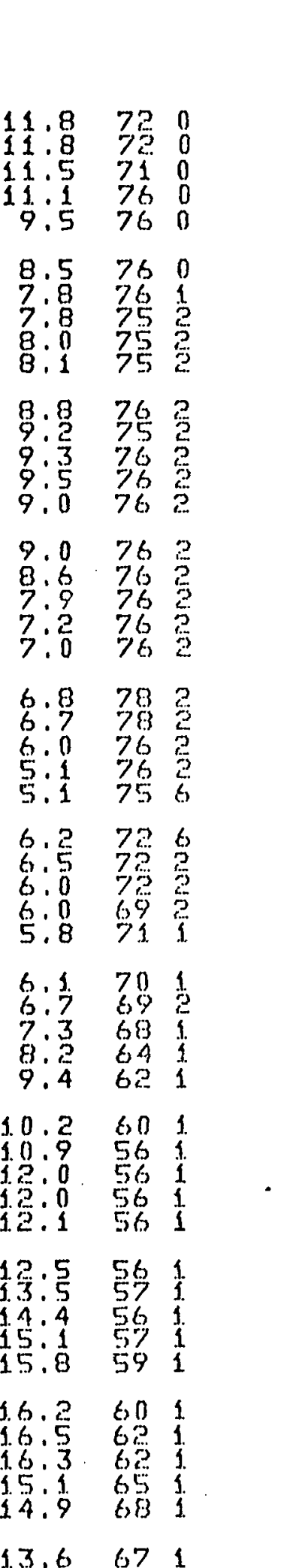

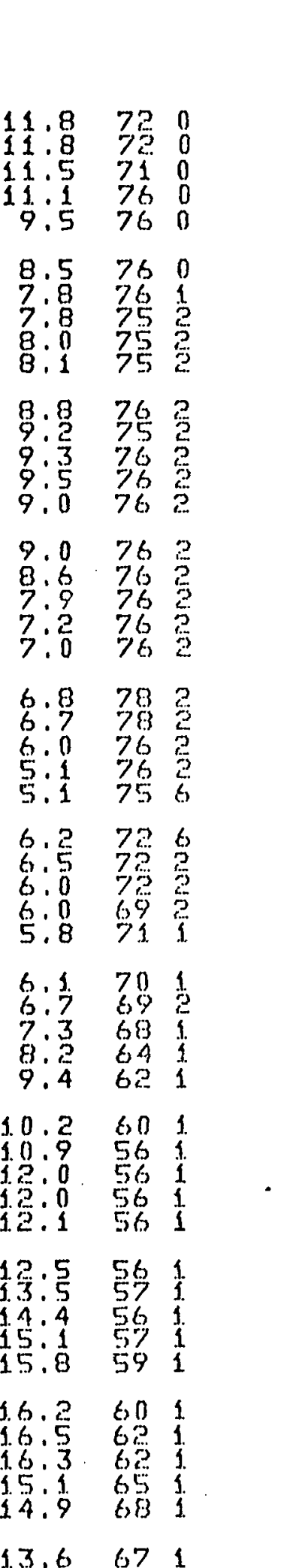

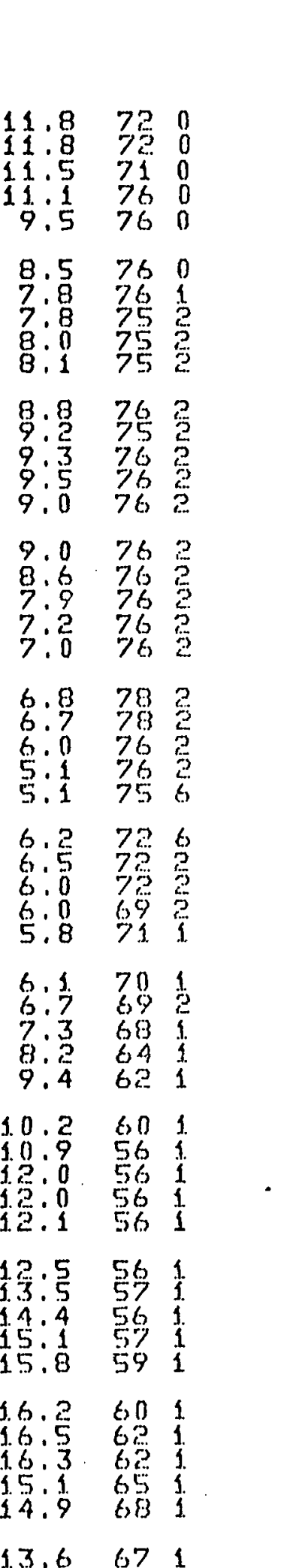

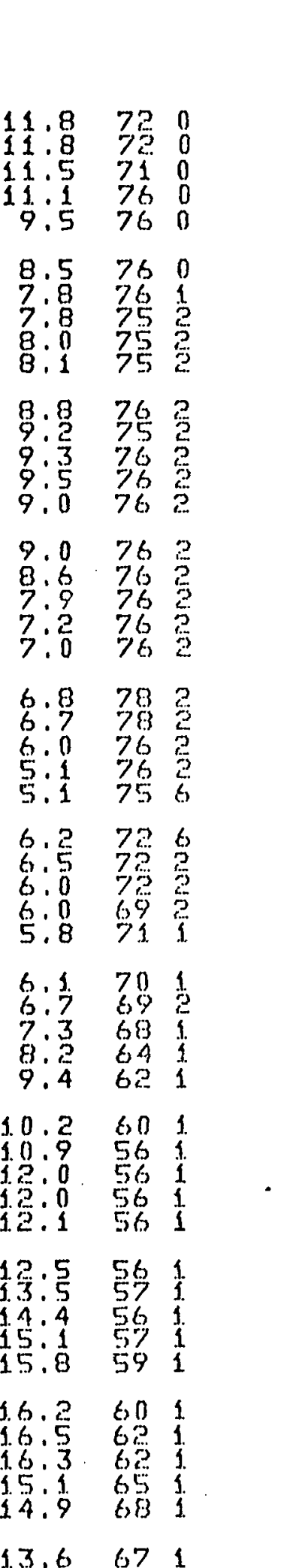

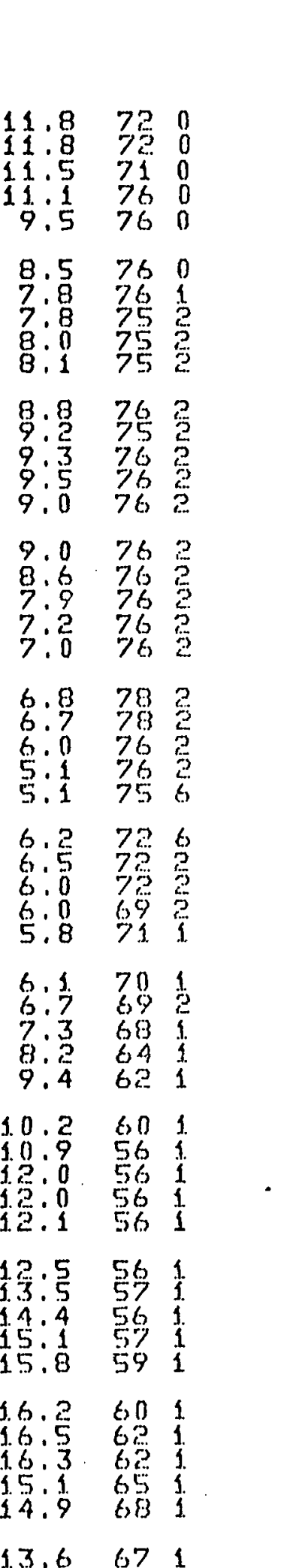

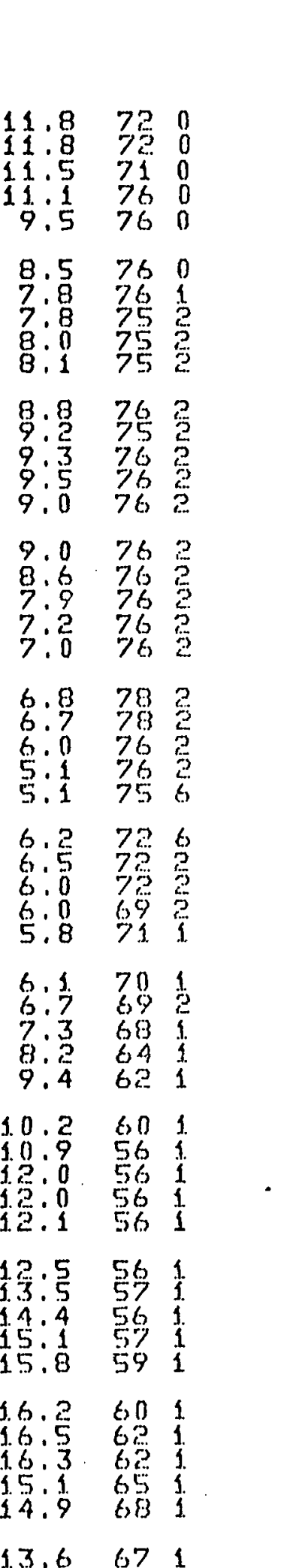

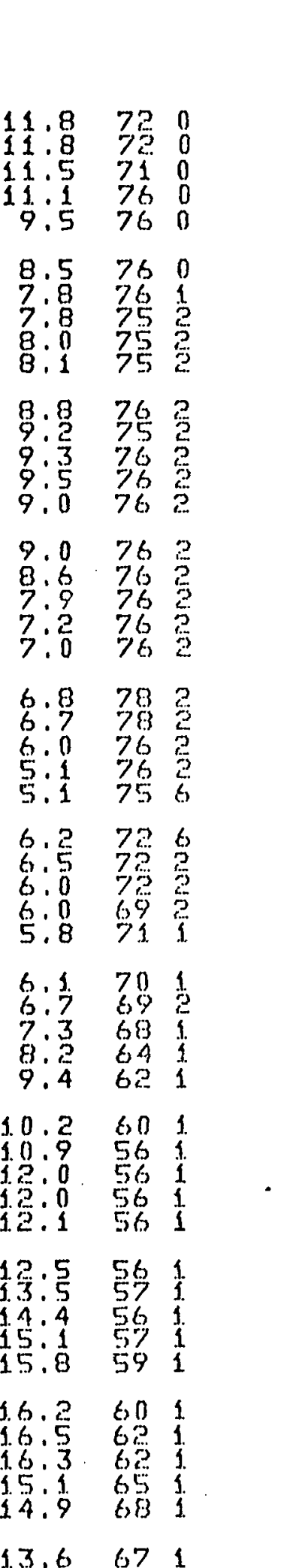

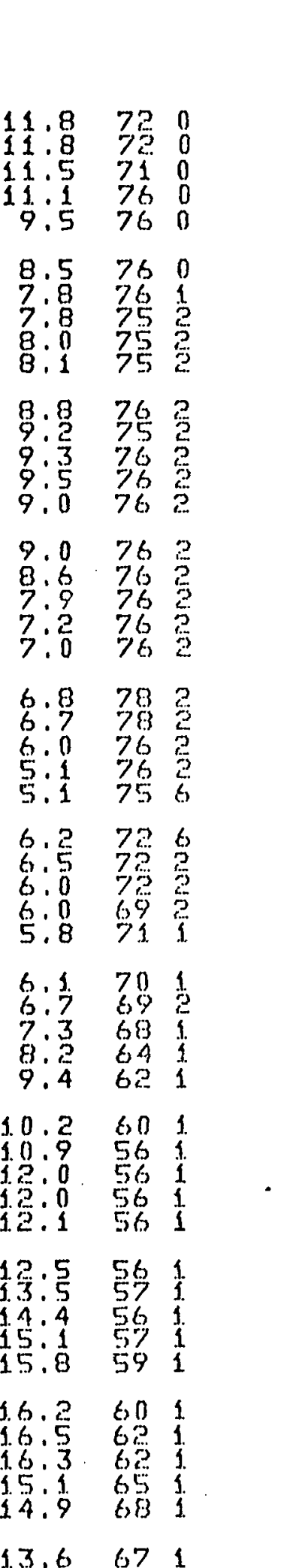

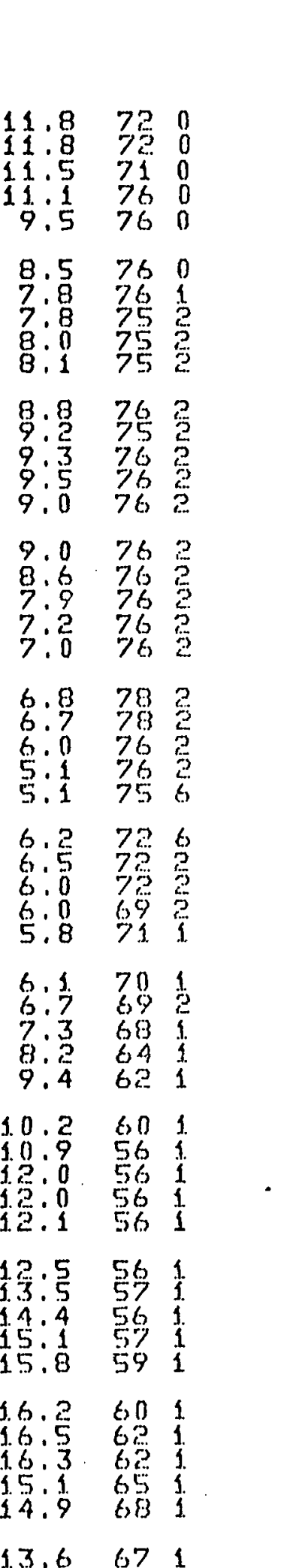

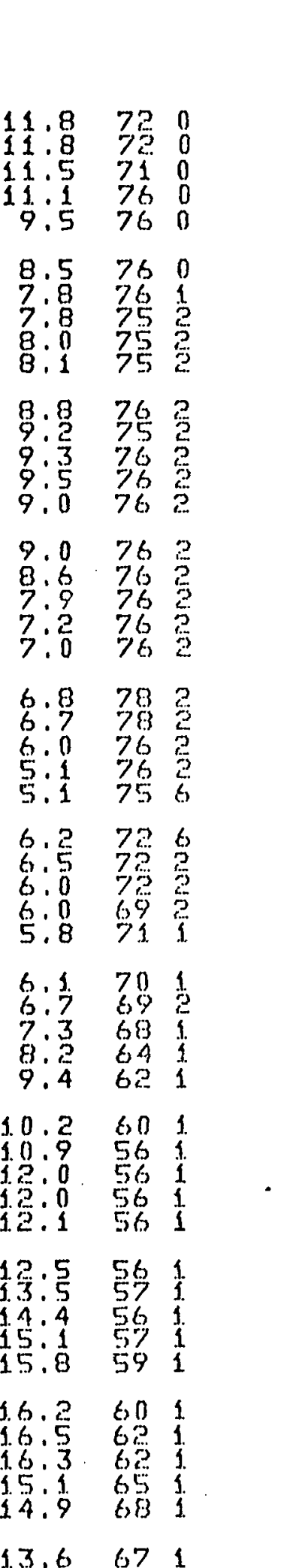

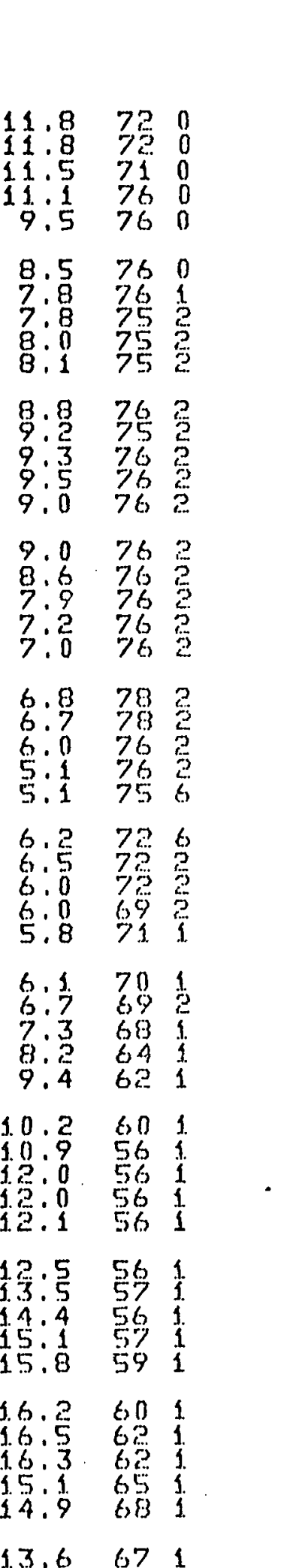

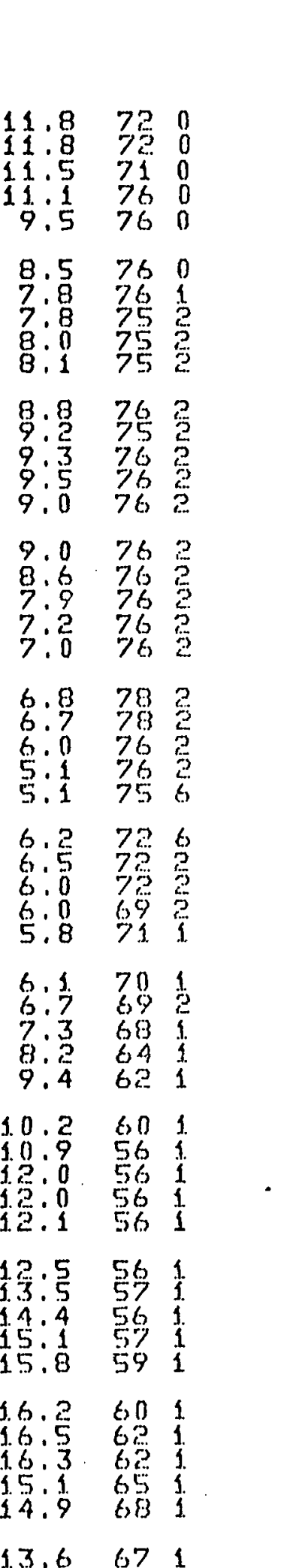

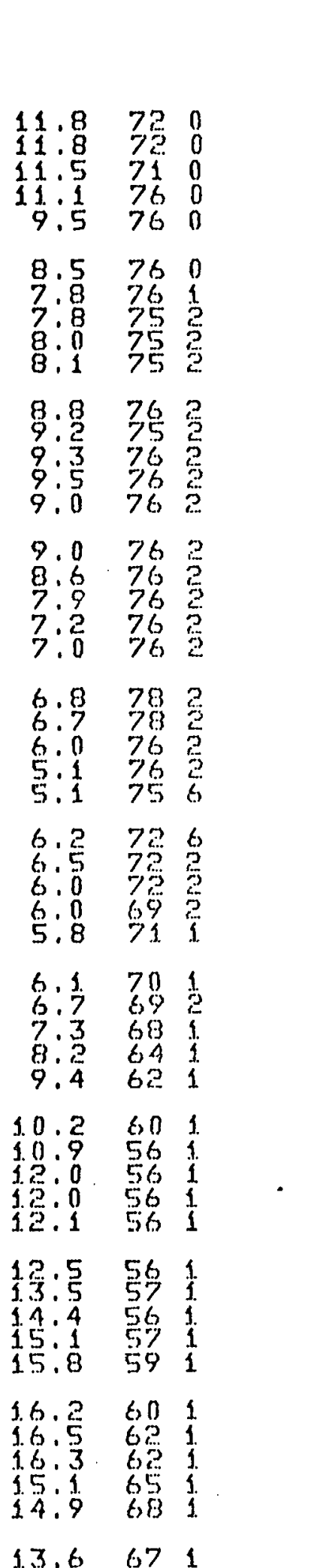

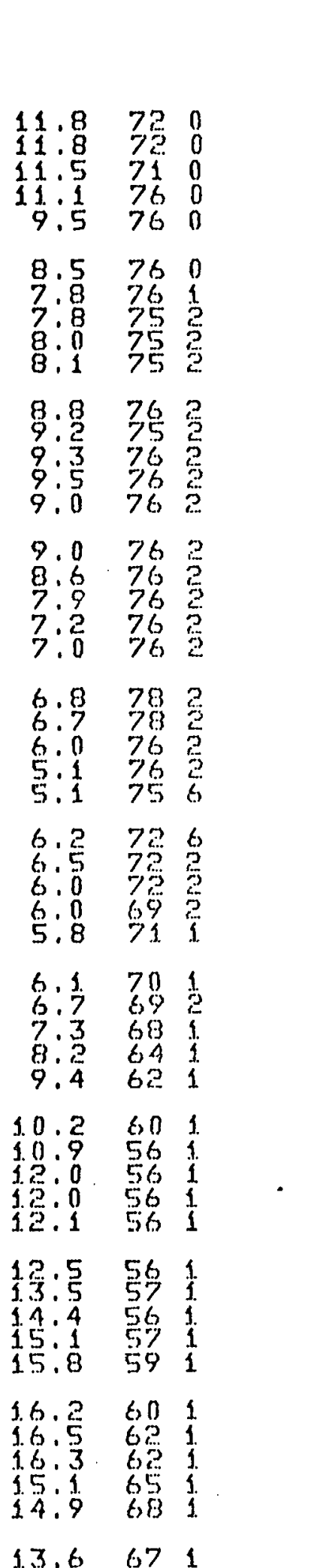

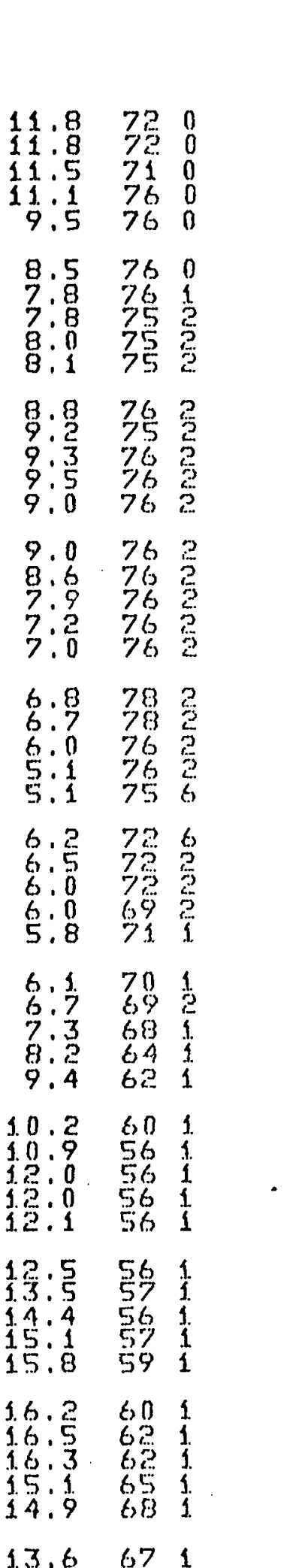

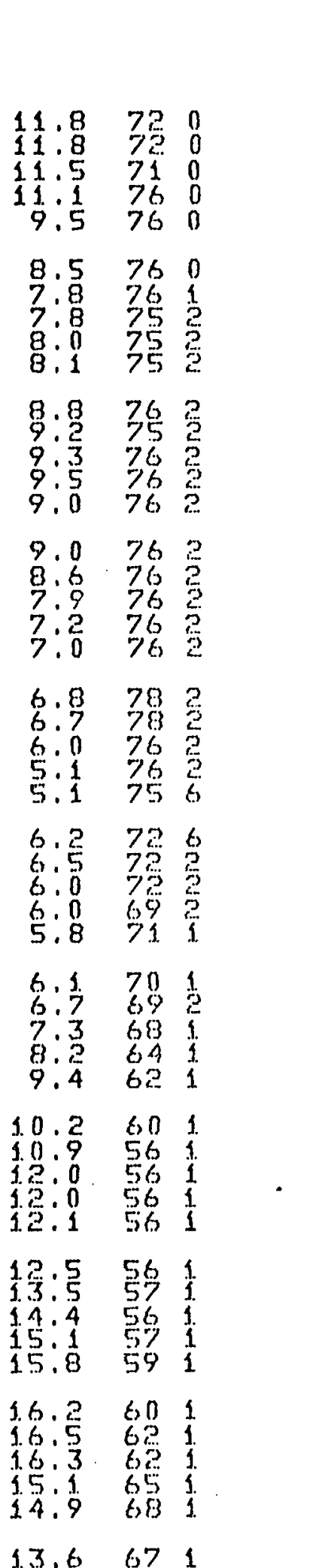

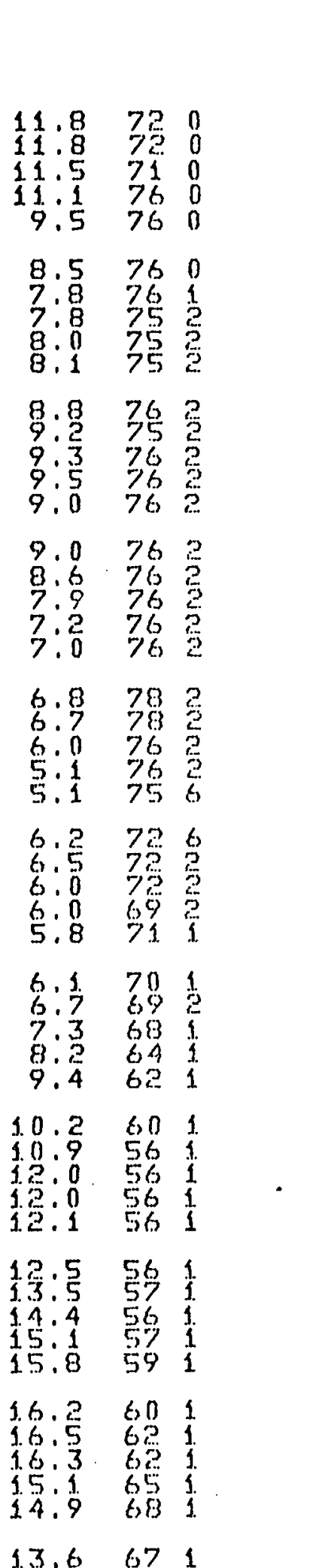

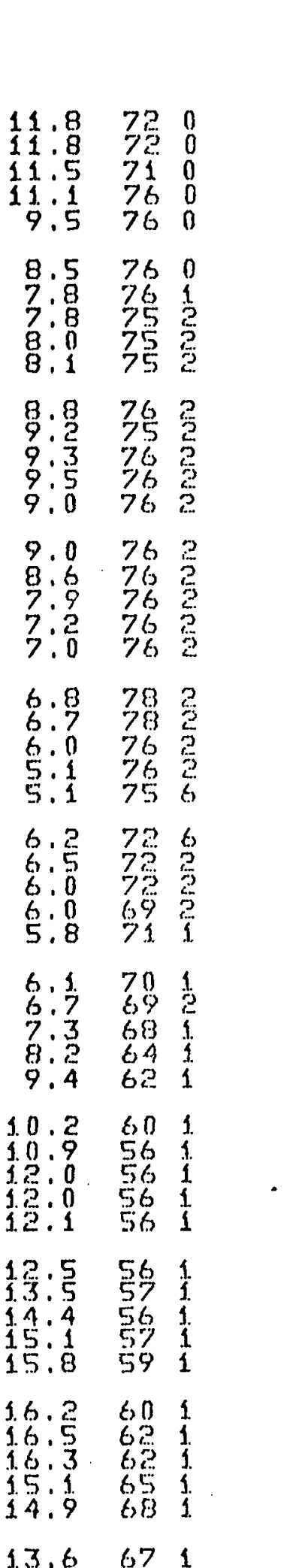

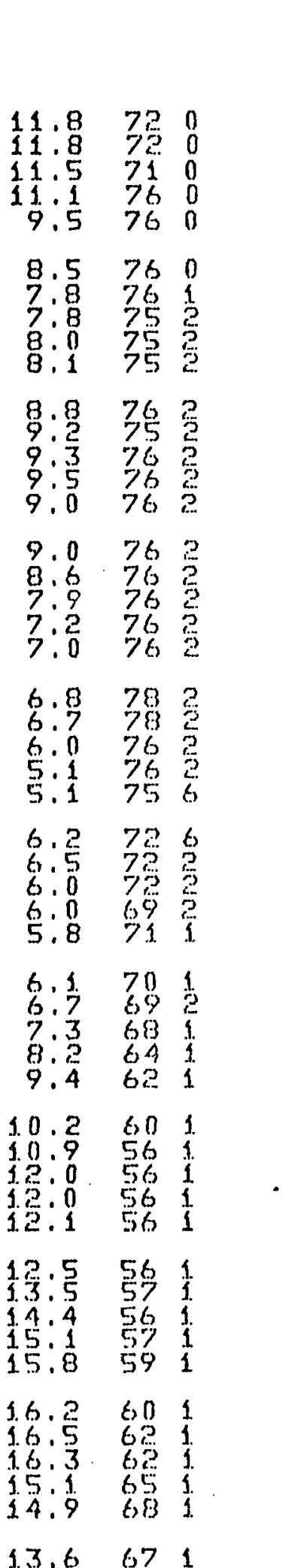

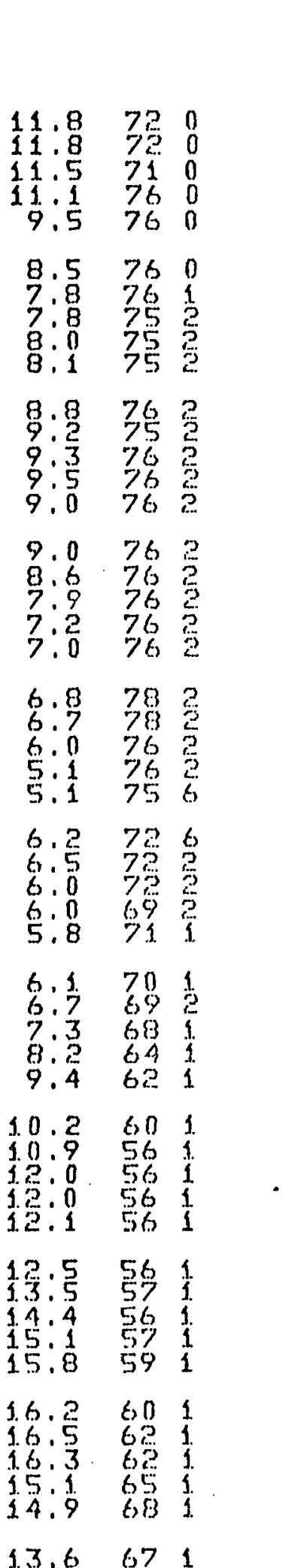

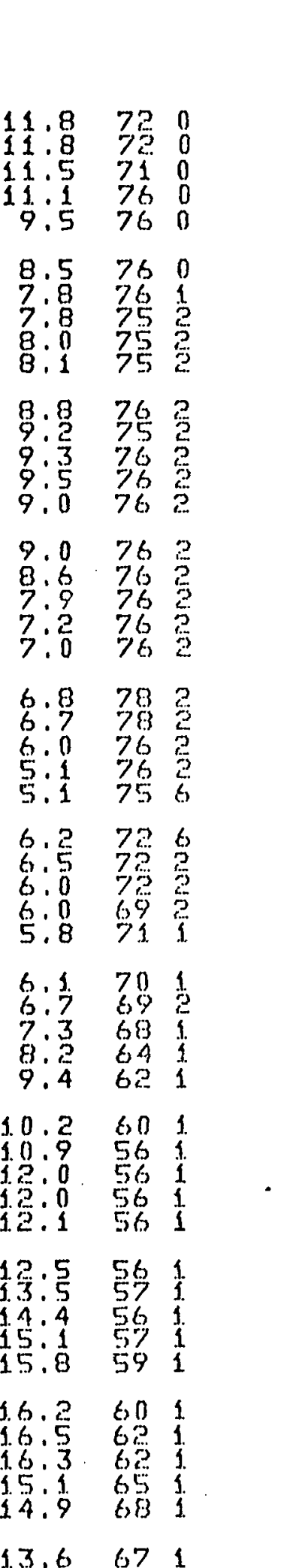

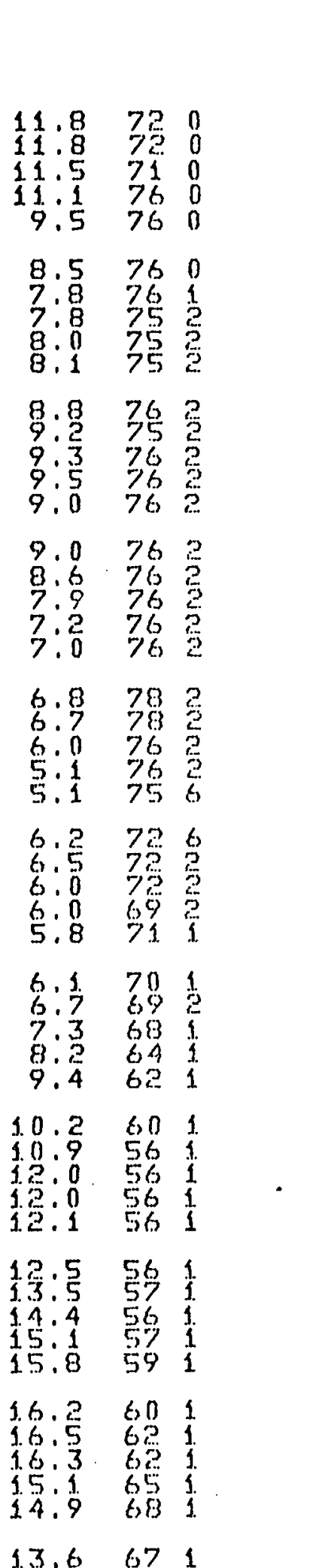

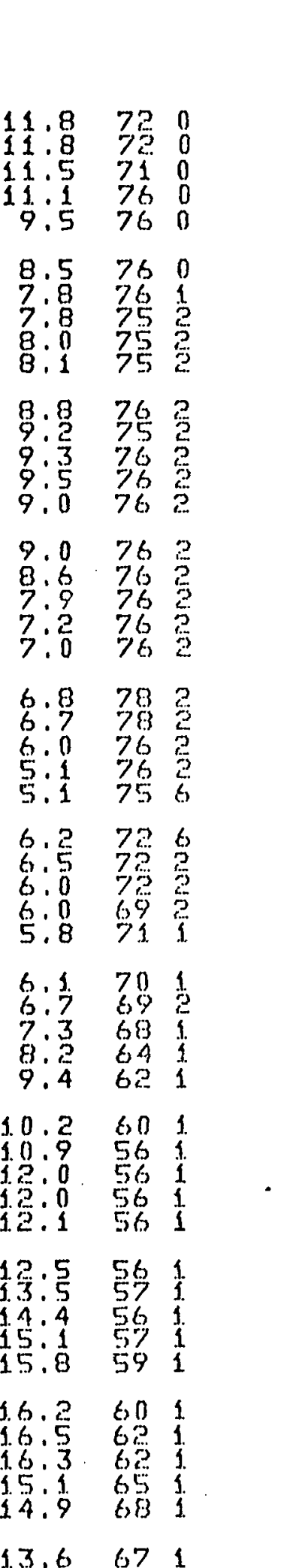

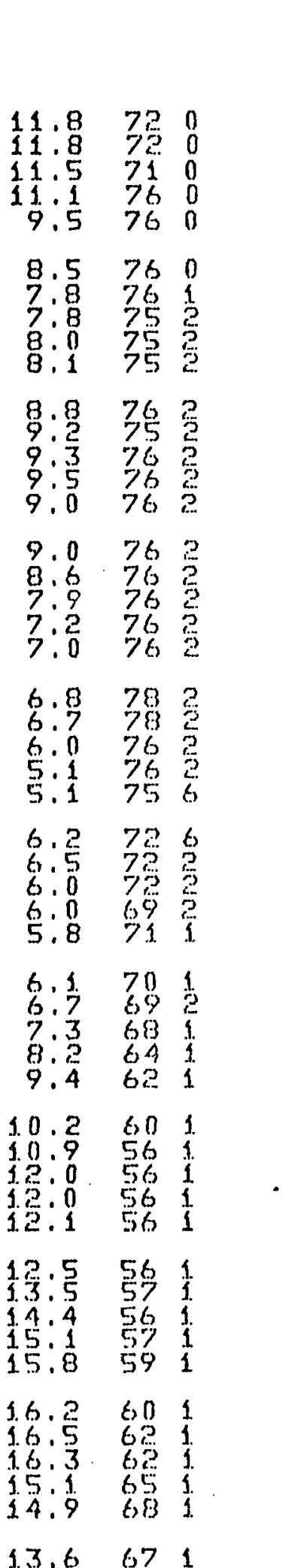

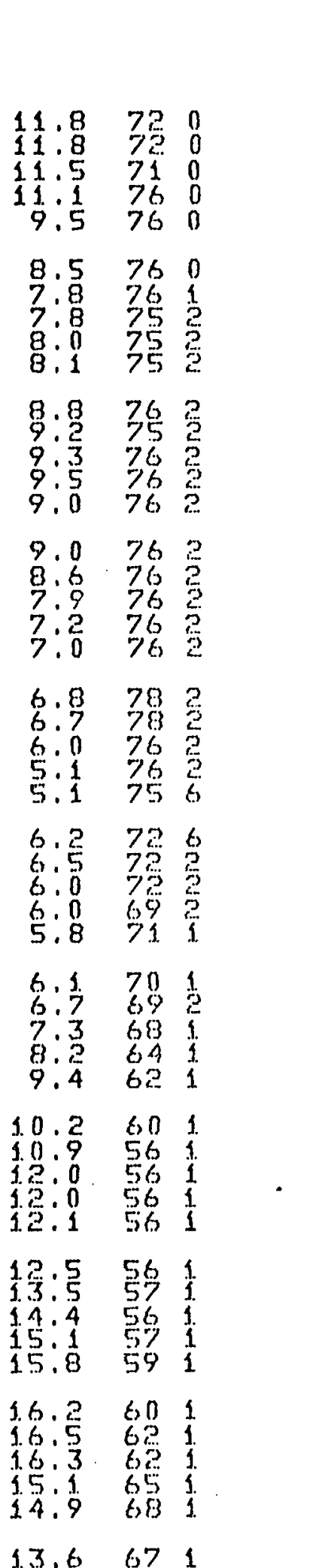

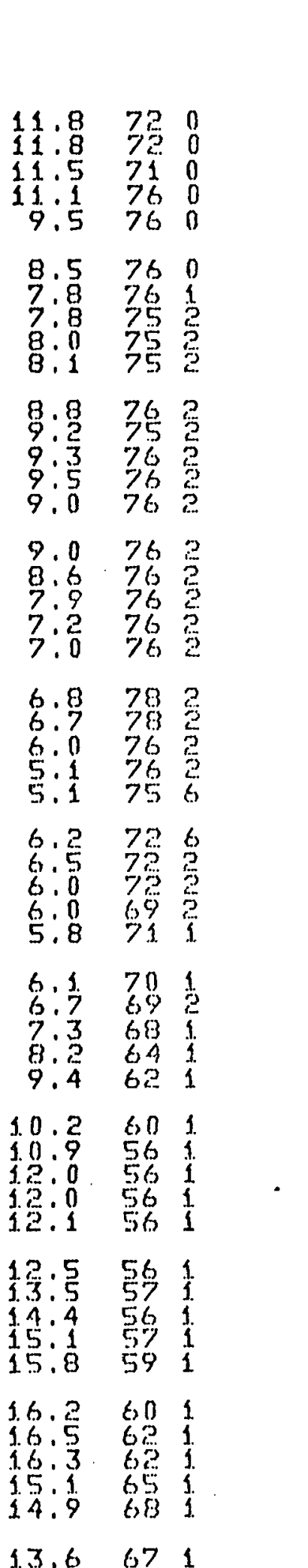

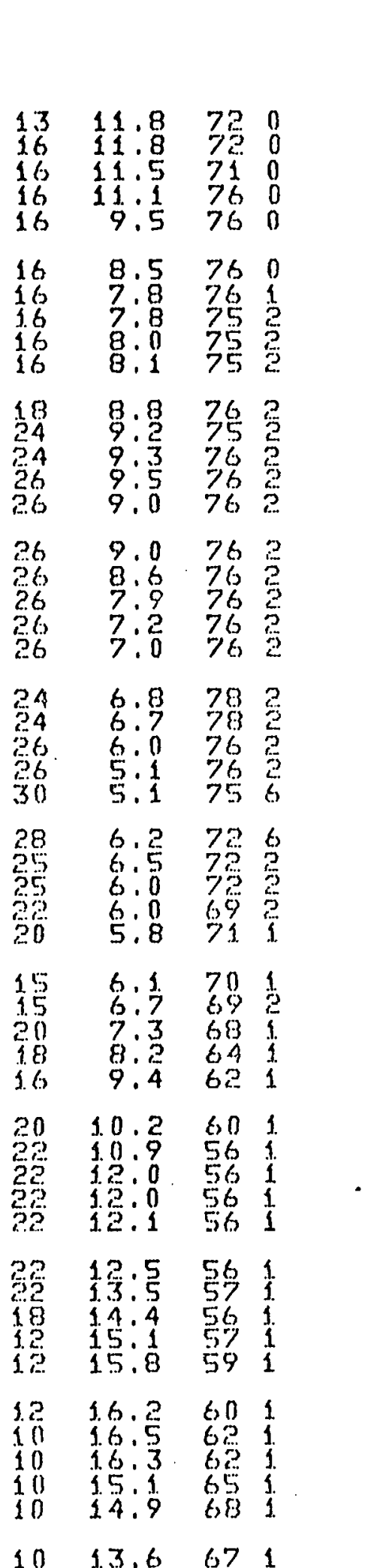

$3.6 \quad 971$

30.60

3. $3.3 \quad 6,8$ 1

$14.4 \quad 681$

16.1670

$16.3 \quad 660$

$3.7 .1 \quad 680$ 
1.31. $131+2919.0 \quad 8041.8316 / 4 / 80 \quad 0700$

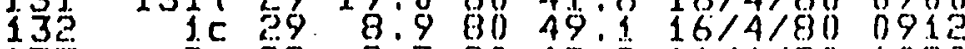

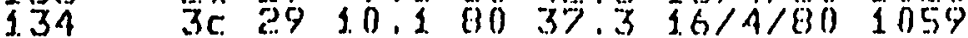
1.35 $4 \times 2911.18030 .5 \quad 16 / 4 / 801201$

136 5c $2912.0 .80026 .016 / 4 / 8301239$ $137 \quad 6 \times 20 \quad 13.0 \quad 8020.0 \quad 36 / 4 / 801354$ $138 \quad 7 c 29 \quad 33.8 \quad 83014.53 .6 / 4 / B 01.435$ $1398 \times 2914.28012 \% 0 \quad 16 / 4 / 80 \quad 1534$

141

3. 42

144

1.45

1.46 $3.4612939 .4 \quad 8010.3 \quad 16 / 4 / 80 \quad 2030$

$14 \% \quad 347+2 \% \quad 46.08030 .516 / 4 / 802100$

$1483148+2952.68010 .4 \quad 16 / 4 / 800030$

349 .36 $300.58010 .416 / 4 / 80$ 231

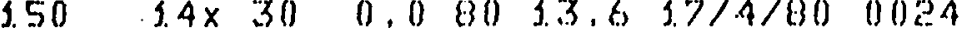

$151 \quad 15030 \quad 0.0 \quad 00 \quad 16.5 \quad 17 / 4 / 80 \quad 0046$

$3.52 \quad 36 \times 30 \quad 0.08039 .537 / 4 / 800154$

153 17c 30

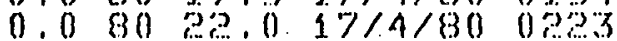

19030

$0.080025 .517 / 4 / 80$ 0351

156

$15 \%$

$20 \times 30$

$0.0 \quad 80 \quad 39.4 \quad 1.7 / 4 / 80 \quad 0537$

1. 58

160

5. 61

1.62

$164364 t+3020.681$

$3.651 .65+3030.986$

$0.18045 .0 \quad 17 / 4 / 800611$

$0.38051,01.7 / 4 / 800700$

$0.1 \quad 8056.837 / 4 / 800736$

$24 \times 30$

0.181

$8.6 \quad 17 / 4 / 80 \quad 090 \%$

$25 c 30$

$5 \cdot 1$

$7.93 .7 / 4 / 803000$

$6.917 / 4 / 80$ 1.100

$5.53 .7 / 4 / 80300$

$5.137 / 4 / 80 \quad 1300$

$166 \quad 16613039.181$

$36 \% 167+3047.581$

$3.6830 .68130 \quad 55.481$

$5.0 \quad 177 / 4 / 80 \quad 1.400$

$5.0 .17 / 4 / 801500$

$3.6,37 / 4 / 803000$

1.70

$17 \%$

173

$1 \% 4$

3.75

1.76

177

1.78

1. $7 \%$

$27 \times 30.54 .8 \quad 80$

$58.3 .57 / 4 / 80$ 17\%

$28 \mathrm{C} \quad 30 \quad 53.5 \quad 80 \quad 5 \%, 4 \quad 17 / 4 / 80 \quad 1755$ $29 \times 30$ 52. $80046.937 / 4 / 801915$ $30 \mathrm{C} 3051.0 \quad 8041.2017 / 4 / 80 \quad 1958$ $34 \times 3050,0 \quad 80 \quad 35.7 \quad 3.7 / 4 / 80 \quad 2105$ 32. $3048.78030 .0 \quad 1 \% / 4 / 80$ 2.48

$33 \times 30 \quad 47.6 \quad 80 \quad 34.4 \quad 17 / 4 / 80 \quad 2308$ $34 \mathrm{C} \quad 30 \quad 46.0 \quad 80 \quad 19,0 \quad 17 / 4 / 80 \quad 3346$ $35 \times 3045,50016.030 / 4 / 0000033$ $36 \mathrm{C} \quad 30 \quad 45.0 \quad 8013.0 \quad 18 / 4 / 8000058$ $37 \times 3044.580 \quad 10.4 \quad 38 / 4 / 80 \quad 0150$

181

3. 8.3

184

185

38030

$39 \times 3043.790$

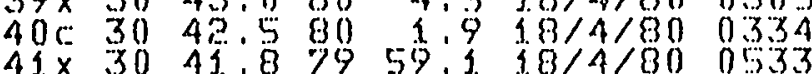

$426 \quad 3041.27956 .3 \quad 18 / 4 / 800557$

1.86 $13.3 \quad 3 \times 29 \quad 9.38042 .816 / 4 / 801020$ $14090 \quad 29 \quad 3.5 .280 \quad 9.8 \quad 36 / 4 / 830 \quad 1553$

$154 \quad 18 \times 30$

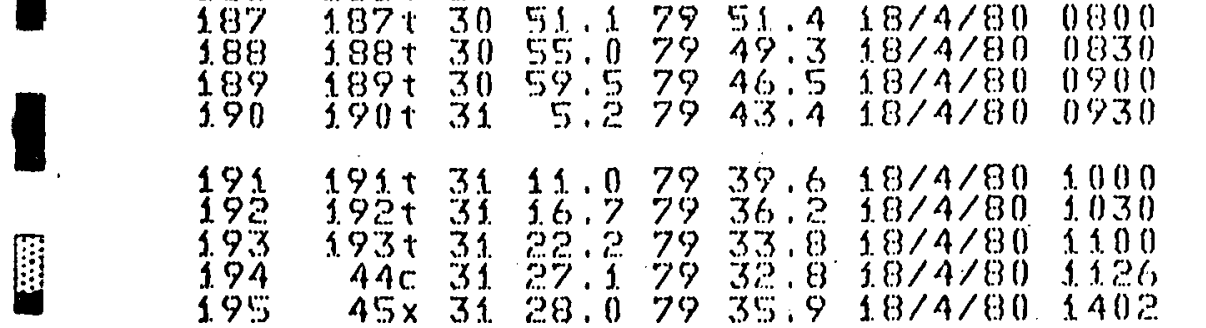

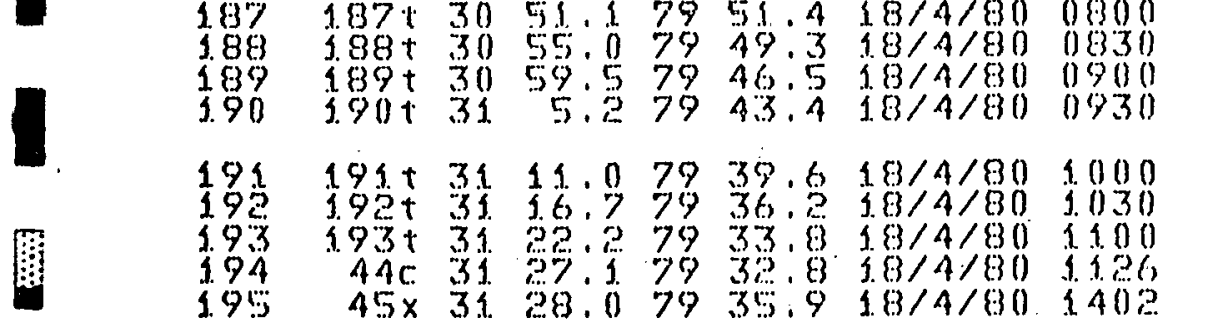

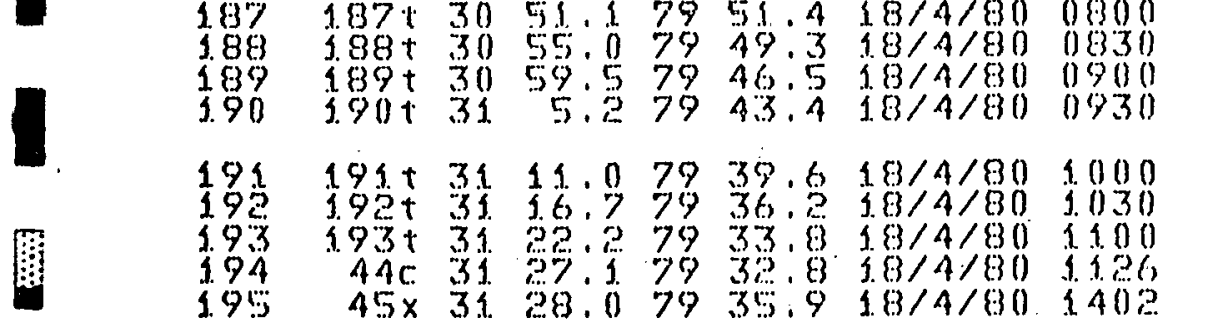

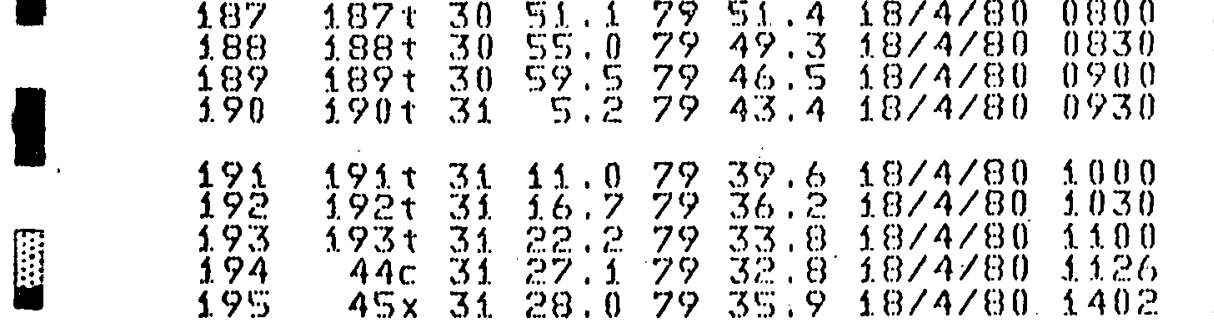

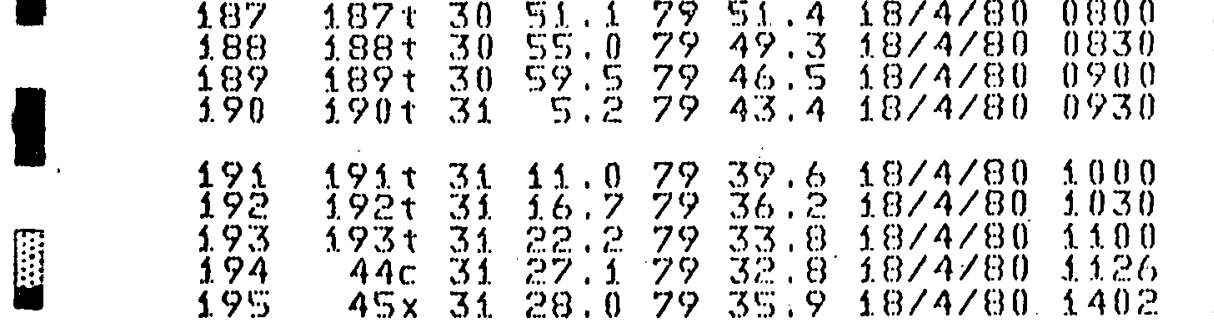

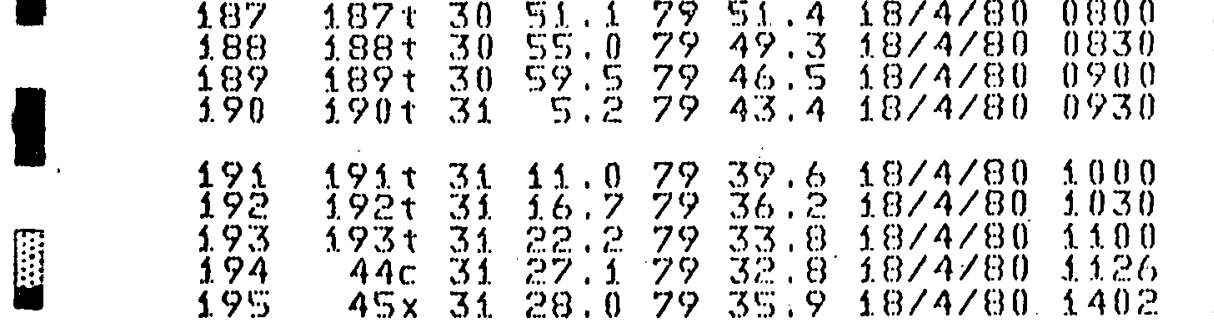
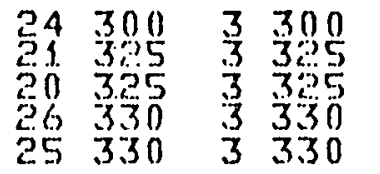

20
12
12
12

$\begin{array}{lll}19.3 & 67 & 0 \\ 20: 2 & 79 & 0 \\ 21: 2 & 66 & 0 \\ 21.4 & 74 & 0 \\ 21.0 & 65 & 0\end{array}$

$31.33 n$

$34 \quad 330$

$54 \quad 330$

$\begin{array}{ll}3 & 3.30 \\ 3 & 3.30 \\ 3 & 330 \\ 3 & 3.30 \\ 3 & 3.30\end{array}$

88330

$153 \quad 3.30$

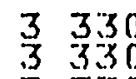

382350

358350

$3 \quad 350$

262350

3350

208350

3350

$272 \quad 35$

2 355

3.59590

2
5

$68 \quad 590 \quad 5 \quad 590$

45500

46590

$\begin{array}{ll}40 & 290 \\ 39 & 315\end{array}$

2290

$\begin{array}{ll}300 \\ 3 & 345\end{array}$

35265

2265

34310

31 .

$\begin{array}{ll}25 & 270 \\ 24 & 270\end{array}$

2340

$\begin{array}{lll}2 & 270 \\ 3 & 370\end{array}$

突

19320

2300

22 320

$26 \quad 335$

335

18

18

1.

160

2 20

13

20.9

650

$12 \quad 20.5 \quad 660$

10

3.1)

19.9

6.9) 0

$10 \quad 19.5 \quad 6830$

$\begin{array}{llll}15 & 19.7 & 68 & 0 \\ 15 & 19.6 & 8.8 & 0\end{array}$

$12 \quad 39.6 \quad 670$

8

19.8 be 0

9

660

8

$20.4 \quad 650$

6

$21.0 \quad 850$

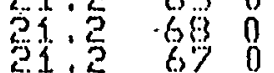

$5 \quad 21,0$

670

30.4

670

$20.5 \quad 650$

4

20.6

650

12

20.9

650

8

24.4

6,50

ㄴ. 5

$6 \% 0$

$25.2 \quad 9803$

$\begin{array}{lllll}24 & 40 & 3 & 40 & 1 \\ 26 & 50 & 3 & 50 & 1 \\ 34 & 70 & 3 & 70 & 1\end{array}$

$\begin{array}{lllll}35 & 70 & 3 & 70 & 130\end{array}$

38110 2 110

43 1. 10 2 150

43110

1210

2 110

$43 \quad 1.10$

3 3.10

8
8
7
7
7

23.4

$25 \cdot 2$

721

631

731

43 1. 1

47 1.0

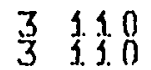

200 90

$\begin{array}{ll}3 & \text { 1. } 50 \\ 3 & 90\end{array}$

$205 \quad 100$

4 1.00

10
30
1.2
13
12
13
17
12
12
8

23.

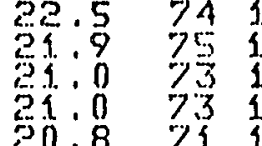

285100

4100

3089

$3\} 1$ 80

380

20.?

681

21.2 681

2.. 0 68 1

$344 \quad 100$

430300

$3 \quad 100$

438 र. 1.

3100

320230
3230

3 250
8
10
15

15

15
16
10

10
1

12

14
14
30.

20.

20.6

20.5

6681

19.5

$19: 0$

19.0

3.8 .4

18.8

1.9 .3

19.8

20.2

20.5

682

682

$\begin{array}{ll}683 & 2 \\ 60 & 5\end{array}$

698 ह

688

688

952

655

70

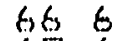

676

686

$20.6 \quad 68$

$\begin{array}{rrr}20.6 & 68 & 2 \\ 20.6 & 68 & 2 \\ 20.2 & 68 & 5\end{array}$

$30.4 \quad 965$

20.9616 
$196 \quad 460 \quad 31 \quad 28.5 \quad 79 \quad 38.5 \quad 18 / 4 / 80 \quad 1438$ $19747 \times 31.29 .67941 .3 \quad 3 / 4 / 80$ 3.5. $1984863129.7 \quad 7944.6318 / 4 / 80 \quad 1619$ $199 \quad 49 \times 3130.979 \quad 47.0 \quad 3.8 / 4 / 803.718$ $20050 \mathrm{c} 31 \quad 31.17949 .8 \quad 18 / 4 / 80 \quad 1740$

201. $51 \times 31 \quad 31.879 \quad 52.7 \quad 18 / 4 / 80 \quad 3813$ 202 52. $3133.47955 .418 / 4 / 801828$ $203 \quad 53 \times 31 \quad 34.98000 .3 \quad 18 / 4 / B 01918$ $204 \quad 546 \quad 31 \quad 36.580 \quad 5.8318 / 4 / 801949$ $205 \quad 55 \times 31.38 .08011 .43 .8 / 4 / 802015$

$206 \quad 56031 \quad 39.4 \quad 80 \quad 17.0 \quad 18 / 4 / 802420$

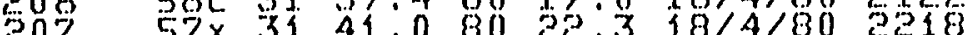
208 58c $3142.68058 .0018 / 4 / 805250$ $209 \quad 59 \times \quad 31 \quad 44.260 \quad 33.5 \quad 18 / 4 / 80 \quad 2354$ 210 600 31 46.0 $80.39 .3019 / 4 / 800033$

21. 63. $63.47 .4 \quad 8044.939 / 4 / 8300134$

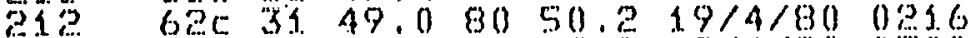
213 2934 31 46.5 $80499.9 \quad 19 / 4 / 800300$ 214 314t $31 \quad 36.9 \quad 8051.835 / 4 / 800400$ 2.15 $515+31$ 27.20 60 5.3.6 $19 / 4 / 800500$

216 210t 31. $17.98056 .7 \quad 19 / 4 / 800600$

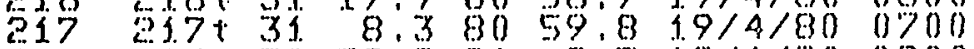
$218318+3059.281 .2 \% 9 / 4 / 800800$

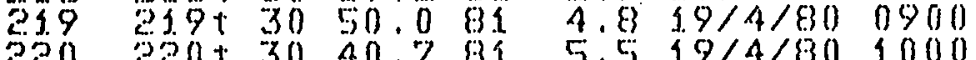

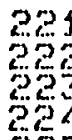

25

2

208

2.30

231

23

234

235

236

2.38

239

2.4 .1

242

2434

245

346

248

349

250

25

25

254
$221+30.30 .881$

$223 \times 3021.481$

$253+30<4.581$

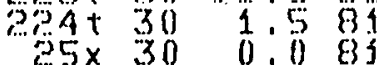

$5.5 \quad 1.9 / 4 / 80$ 1.1.00

6. $19 / 4 / 80$ 1200

$7.19 / 4 / 803.300$

$8.259 / 4 / 801400$

$8.439 / 4 / 60 \quad 3.435$

$24 \times 30$

$23 \times 30$

$25 x=30$

$0.080 \quad 56.75 \% 19 / 80 \quad 3608$

$0.18050 .8319 / 4 / 80$ 1.716

$20 \times 30$

$0.18039 .319 / 4 / 801905$

$19 \times 30$

$0.280 \quad 33.5 \quad 19 / 4 / 801.947$

$18 \times 30$

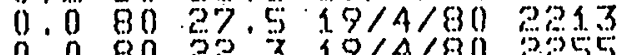

$3.7 \times \quad 30$

$0.08019 .520 / 4 / 800005$

$3.5 \times 30$

$0.08016 .50 / 4 / 800026$

$14 \times 30 \quad 0.0 \quad 80 \quad 13.520 / 4 / 80 \quad 014 \%$ $238+2959.78014 .350 / 4 / 800400$ $539+29950.08017 .450 / 4 / 800500$ $340+2943.580$ 2.1.5 $20 / 4 / 800600$

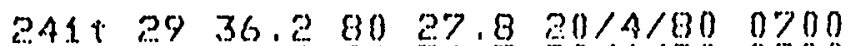
$342+2928,48034.550 / 4 / 800800$ $243+2920.38040 .550 / 4 / 800900$ 344 t $398.48046 .320 / 4 / 801000$ 3. $2.28 \quad 8.0 \quad 8048.750 / 4 / 80 \quad 3034$

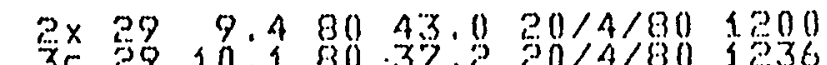
$4 \times 291.1 .08031 .520 / 4 / 80 \quad 1345$

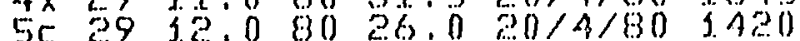
$250+2921.5 \quad 80 \quad 35.920 / 4 / 80 \quad 1600$

$251+2926.18026 .820 / 4 / 801.700$ $25 \%+2920.58024 .820 / 4 / 801800$ $6 \times 2913.080$ 20.3 $20 / 4 / 801857$

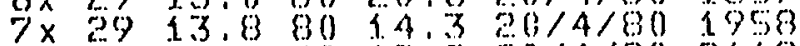
$8 \times 2914,6 \quad 80$ 12.2 $20 / 4 / 80$ 213
$0.081 \quad \therefore .5 \quad 19 / 4 / 80 \quad 1517$ $3.3 \times 30.0 .080$ 1.0.5 $20 / 4 / 8000208$
144

E3ध

$50 \quad 6$

$47 \quad 80$

4780

4260

$\begin{array}{ll}40 & 70 \\ 39 & 70\end{array}$

3790

$28 \begin{array}{ll}200 \\ 28 & 105\end{array}$

$\begin{array}{ll}25 & 1 \\ 2 & 1 \\ 20 & 1.5 \\ 18 & 90\end{array}$

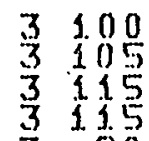

1390

1300

1.490

3.590

15

1.8

20

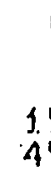

$\therefore 4 \quad 4$

26

1.8

$\begin{array}{ll}23 & 3 \\ 25 & 4 \\ 3 & 4\end{array}$

3.34

$\begin{array}{rr}41 & 50 \\ 43 & 100 \\ 45 & 95 \\ 50 & 95 \\ 68 & 310\end{array}$

176 140

287

49

44

$\begin{array}{ll}20 & 50 \\ 26 & 60\end{array}$

?6 60

2125

$24 \quad 340$

$36 \quad 340$

$3-3 \quad 340$

2340

$2 \% 340$

29340

34340

54340

$75 \quad 350$

3830

360

3110

$\begin{array}{rr}3 & 110 \\ 3 & 90\end{array}$

350

4340

4340

\section{0}

3340

4340

1350 $\begin{array}{ll}3 & 30 \\ 3 & 60 \\ 3 & 60 \\ 3 & 60 \\ 3 & 80\end{array}$

$\begin{array}{rrrr}10 & 20.0 & 61 & 6 \\ 8 & 19.8 & 61.2 \\ 8 & 19.7 & 61 & 2 \\ 8 & 99.4 & 6.3 & 2 \\ 10 & 19.4 & 63 & 5\end{array}$

$\begin{array}{lll}3 & 70 & 12\end{array}$

11
15
12
17
12

19.1

18.8

18.8

18.8

645

641

641

65

19.1661

$9.0 \quad 61$

$18.6 \quad 641$

18.2631

39010

18.0

6.31

18.0 .634

$17.0 \quad 6.31$

$16.8 \quad 641$

1.6.7 64 1

1.7.0

637

17.3

17.6

62

1. 8.4

605

18.062

$17.6 \quad 60 \%$

17.0 60?

$35.0 \quad 60 \%$

58

3.4 .2

13.960

$\begin{array}{lll}4 & 30 & 50 \\ 4 & 40 & 30\end{array}$

1. $4: 5$

(6) 6

1.4.1 661

1.4.5 67

$14.3 \quad 672$

a

1.4 .2

68

15.1

3. 4.6

682

$14.2 \quad 672$

13.5 .702

3. 0

15
20
17

13.

$5.4: 0$

779

$13.4 \quad 89$

13.2672

$93.0 \quad 681$

1.4
12
3.3
12

13.3

3.8

701

$14.4 \quad 741$

$\begin{array}{lll}4 & 19 & 50\end{array}$

1.4 .6

13.1

13.0

721

1.2.

1.2.

70

1.4

11.?

6,81

23

1. 4.4

71. 1

18

1.1. 5

6.81

25

1.1. 4

14.2

681

671

20

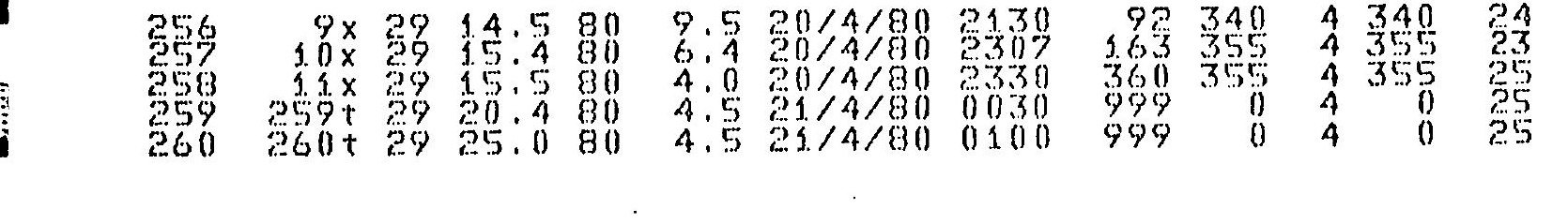

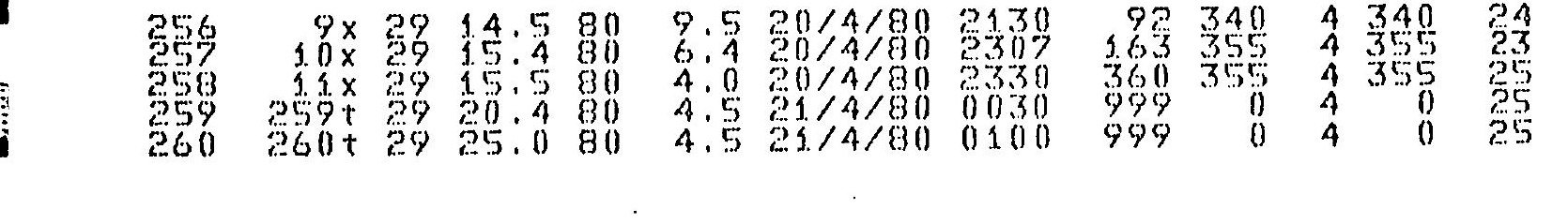

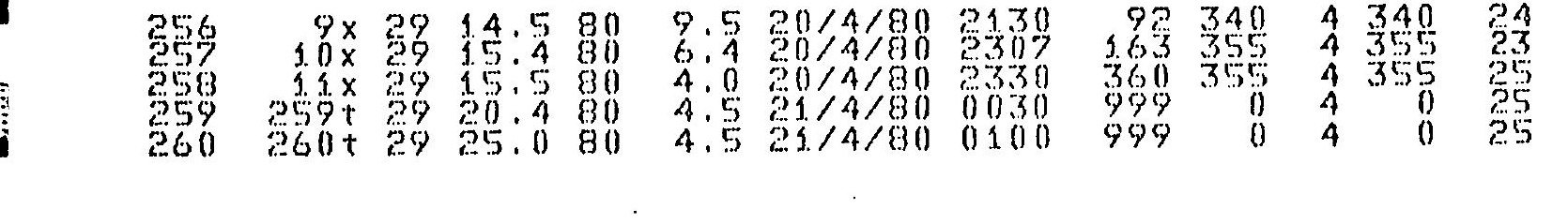

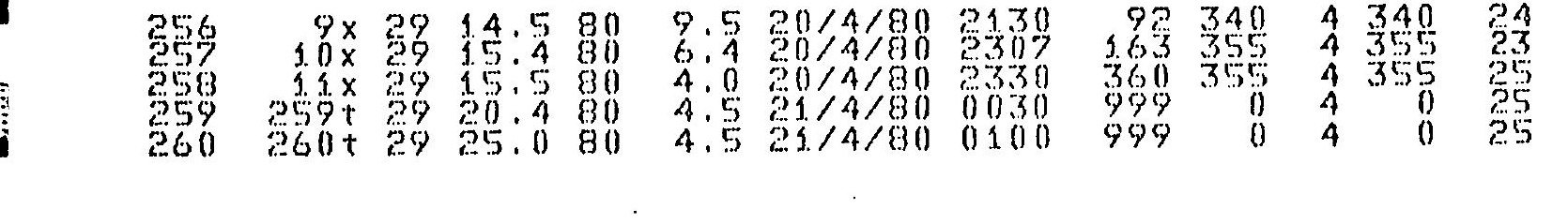

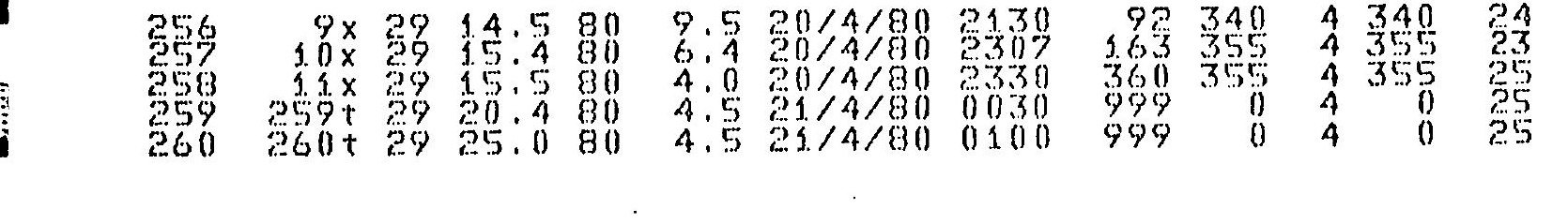

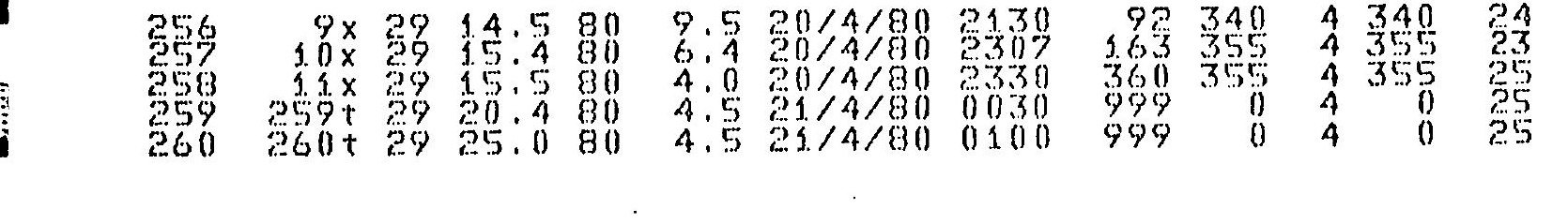

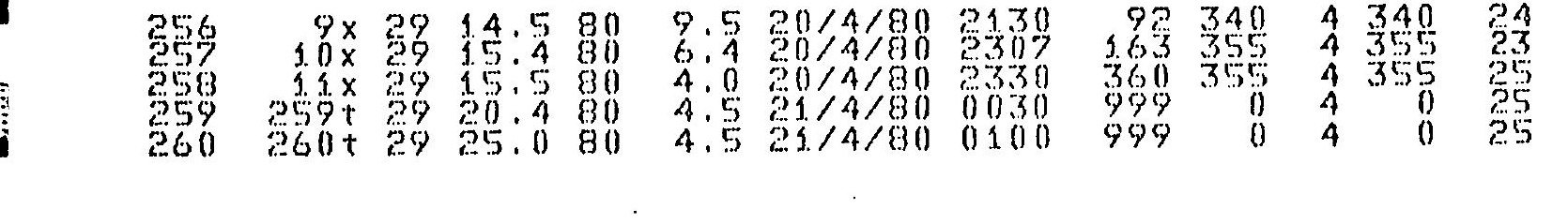

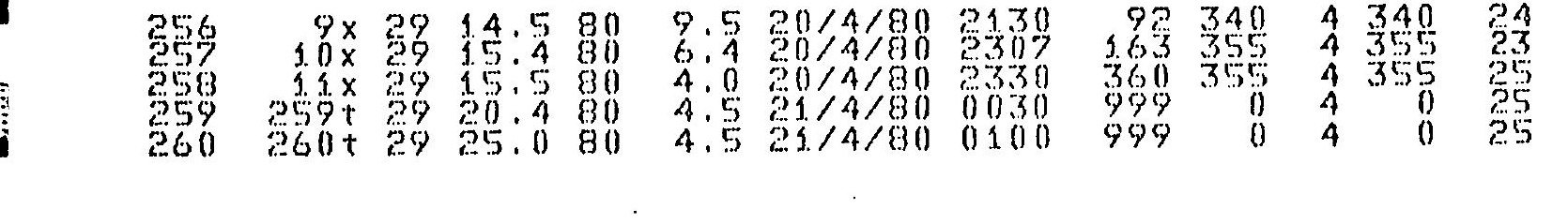

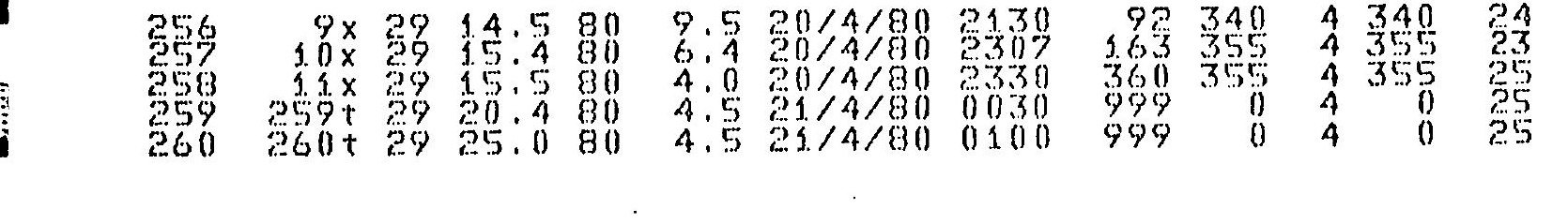

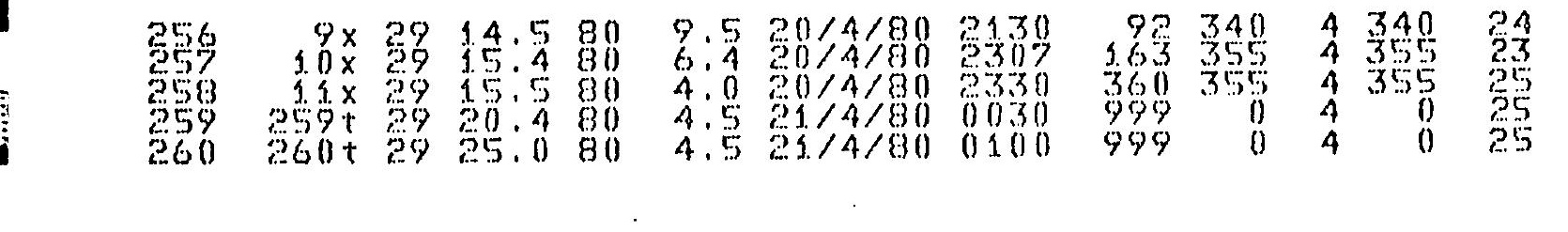

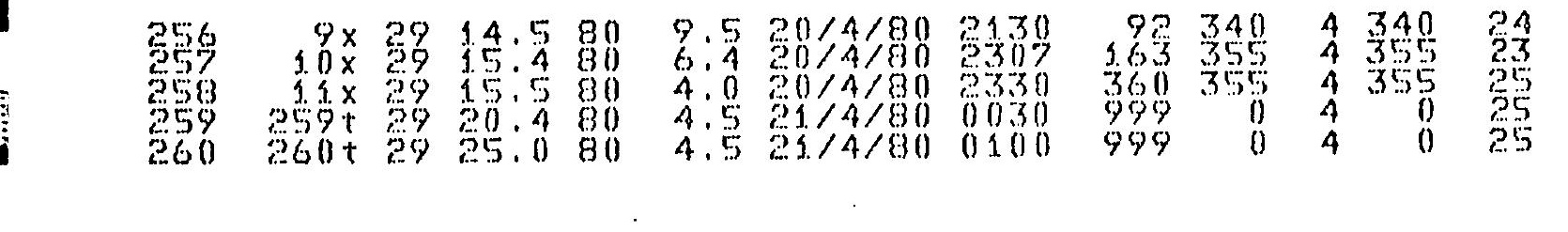

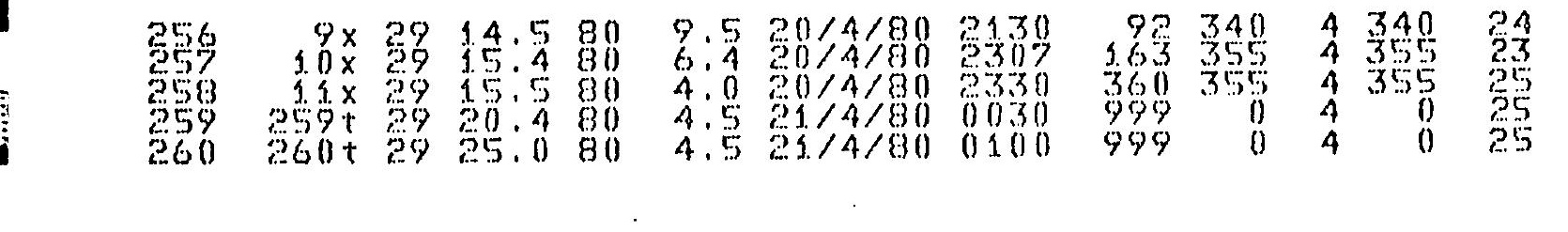

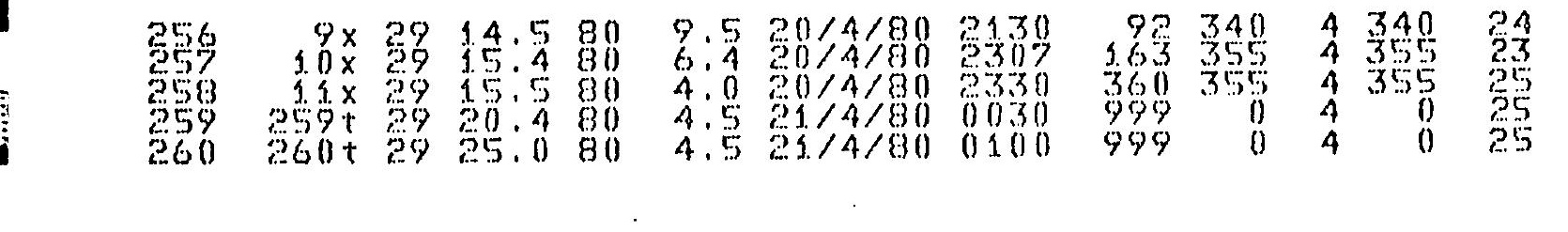

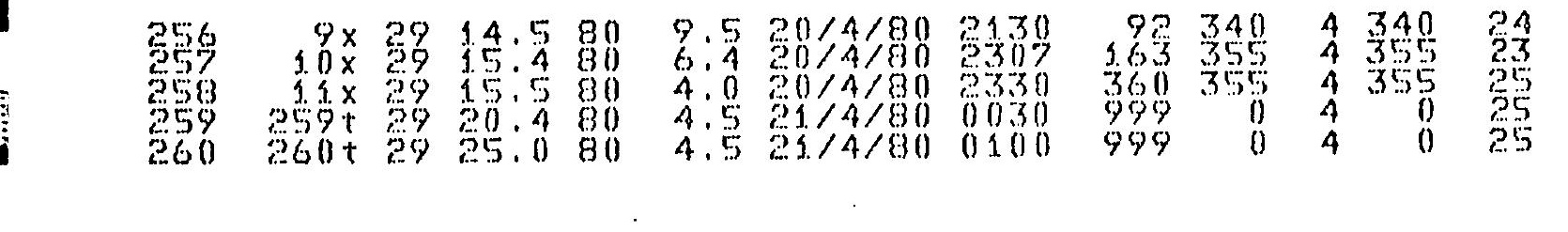

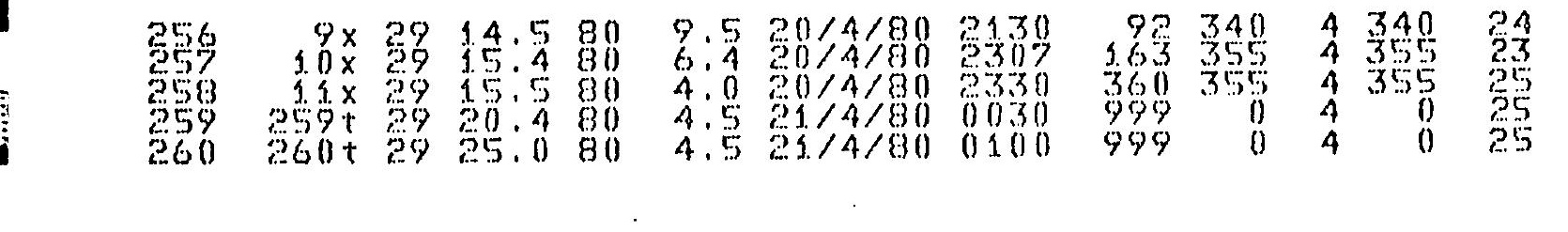

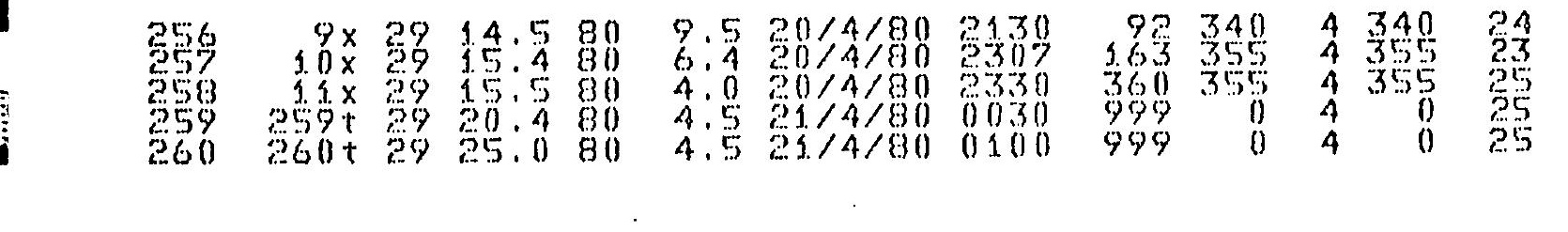

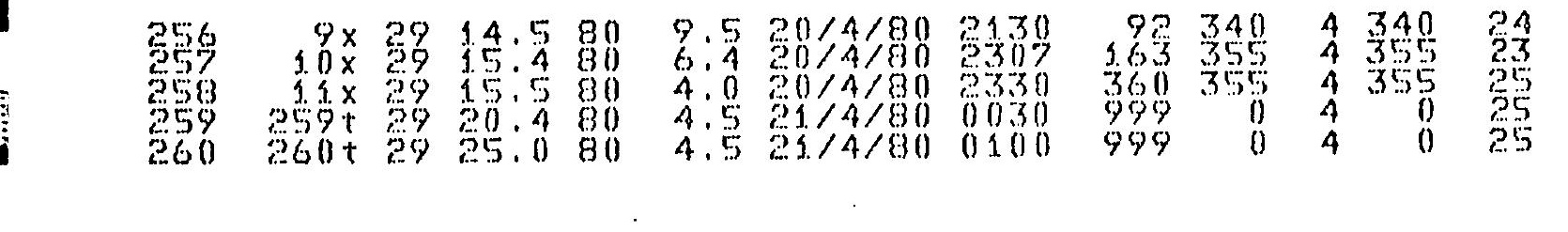

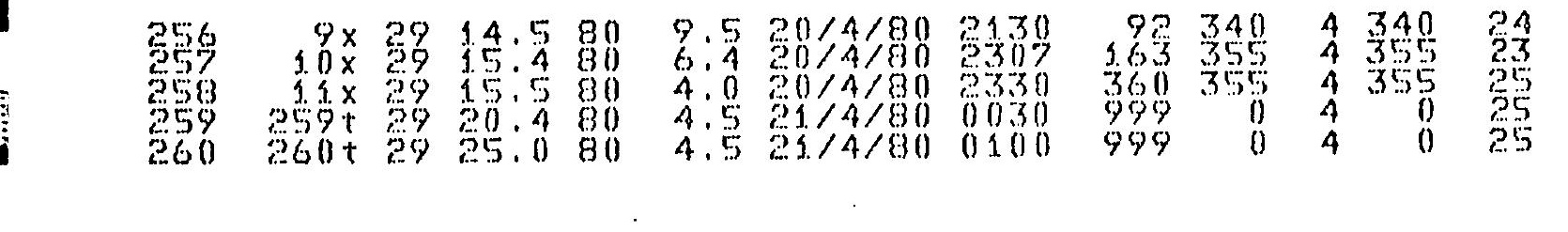


$261 \quad 261+2929.580 \quad 4.921 / 4 / 800130$ 262. $262+2934.180$ 5.2. $21 / 4 / 80$ 0.200 $263 \quad 263+29 \quad 38.5 \quad 80 \quad 6.0 \quad 21 / 4 / 80 \quad 0230$ 264 264t 2942.580 6.5 $21 / 4 / 800300$ $265265+2946.480 \quad 7.221 / 4 / 800330$

$266.266+2950.380$

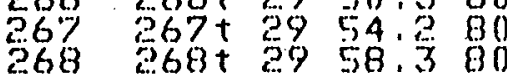

B.2 $25 / 4 / 800400$ $54 \times 30 \quad 0.28003 .6 \quad 31 / 4 / 800551$.

$15 \times 2959.8 \quad 80 \quad 16.321 / 4 / 800609$ $16 \times 300.0800 .9 .3 \quad 3 / 4 / 800656$ $17 x 2959.8 .8020 .51 / 4 / 800712$ $3.8 \times 300.08037 .721 / 4 / 800908$ $19 \times 30 \quad 0.0 .8033 .653 / 4 / 80 \quad 0940$

276

$20 \times 30 \quad 0.08039 .521 / 4 / 803146$ $21 \times 30$ $20 \times 30$

23050 $0.08045 .021 / 4 / 80$ 3.23? 0. $060 \quad 50.7 \quad 21 / 4 / 80 \quad 1429$ $0.08056 .7,31 / 4 / 801508$ 398

28

25030 0.0 .8$. 2.. $6201 / 4 / 80$ 1.6\%

0.081

9.3 $21 / 4 / 80 \quad 1700$

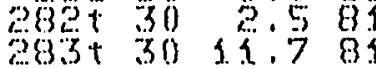
$584+30$ की. 81 $8.3 \quad 31 / 4 / 80$ 3900 283 284 $285+3030.6 \quad 81$

7.4 2.:1/4/80 1900 $6.953 / A / 805000$

$286+3040.081$.

$6.521 / 4 / 802500$

286 $287+3048.081$

$4.831 / 4 / 80 \quad 5300$

360.3056 .283

288

$3.522 / 4 / 80000$ 280.3053 .580 53.5 $52 / 4 / 80005$

28

29

$39 \times 3052.28046 .920 / 4 / 800344$ $30 c \quad 3051.1 \quad 80$ 41.5 $25 / 4 / 80033$ $39 \times 3049.8 \quad 80 \quad 35.723 / 4 / 800506$ $\begin{array}{lllllll}32 \mathrm{C} & 30 & 48.6 & 80 & 30.0 & 22 / 4 / 80 & 0540\end{array}$ $33 \times 30 \quad 47.3 .80$ 24.2 $20 / 4 / 800650$

295

296

$34 C \quad 30 \quad 46.380 \quad 18.925 / 4 / 80 \quad 0 \% 23$

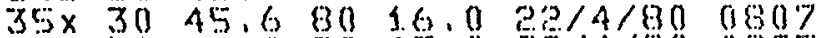

$36,6.3045 .0 \quad 80 \quad 33.0 \quad 25 / 4 / 800827$

$37 \times 3044.5$ 80 $10.45 / 4 / 800000$

300

301

302

304

305

306

$30 \%$

309

310

31

312

31.4

315

316

318

319

320 $38 \mathrm{c}$

$4.5 \quad 22 / 4 / 80 \quad 1023$

$40 \mathrm{c} 30$ 42:9 80 3:0 $25 / 4 / 80$ 3045 $41 \times 3042.0795 \%, 872 / 4 / 801206$

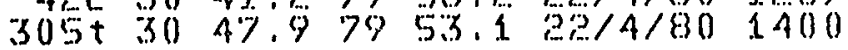

$306+3053.77951 .825 / 4 / 80$ 1.430 $307+3057.079 \quad 48.9 \quad 39 / 4 / 80 \quad 1500$ $308+31$ 2.0 $7946.020 / 4 / 801530$ $309+31 \quad 8.07942 .3 \quad 23 / 4 / 801600$ $310+31 \quad 13.77939 .522 / 4 / 803.630$

$311+31 \quad 19.37936 .528 / 4 / 801700$ $312+31.25 .279 \cdot 34.250 / 4 / 801.730$ $440.3107 .17933 .129 / 4 / 80$ 1746 $45 \times 31 \quad 27.67935 .6 \quad 2 . / 4 / 80$ 1926 $46 \mathrm{C} \quad 31.28 .579 \quad 38.520 / 4 / 80$ 1558

$47 \times 31.08 .579$ A1. $5 \quad 22 / 4 / 80$ 2105

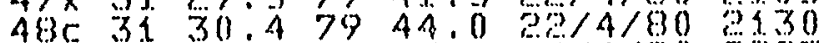

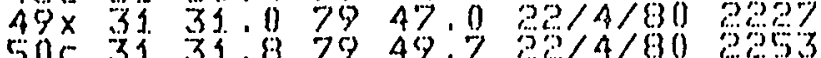

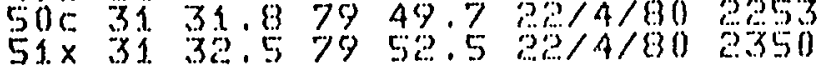

$5203133.17955 .5 \quad 23 / 4 / 800012$ $53 \times 31 \quad 35.0 \quad 8000.523 / 4 / 8000133$

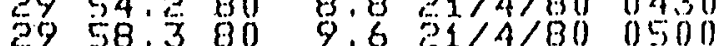
$13 \times 30 \quad 0.0 \quad 8010.451 / 4 / 800517$ $42 \mathrm{c} 3041.279 \quad 56,252 / 4 / 801.23 \%$

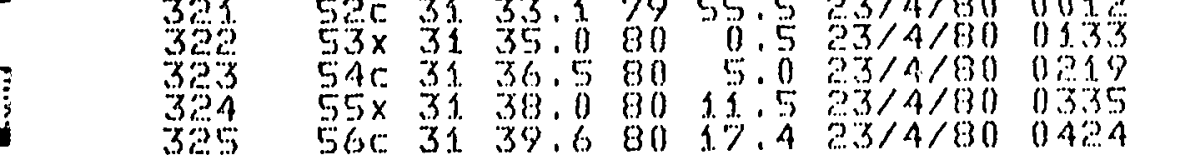

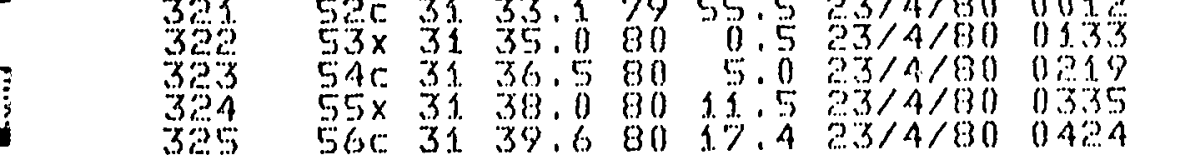

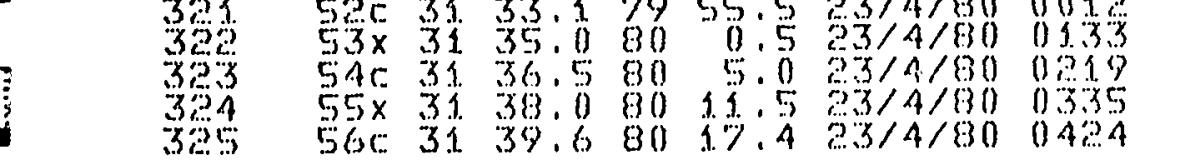

$\begin{array}{lll}999 & 0 & 4 \\ 999 & 0 & 4 \\ 999 & 0 & 4 \\ 999 & 0 & \\ 999 & 0 & 4\end{array}$

$\begin{array}{lll}4 & 0 & 25 \\ 4 & 0 & 25 \\ 4 & 0 & 25 \\ 4 & 0 & 25 \\ 4 & 0 & 25\end{array}$

$9.5 \quad 6891$

$9.1 \quad 681$

$8.9 \quad 6831$

357330

4330

347.330

$\begin{array}{ll}314 & 330 \\ 203 & 330\end{array}$

330

1. $683 \quad 330$

33.30

68330

5330

45320

$42 \cdot 300$

$43 \quad 325$

37300

34320

30300

(1) 80

17890

$\begin{array}{ll}20 & 190 \\ 20 & 315 \\ 20 & 3.5\end{array}$

20315

1.9310

$19 \quad 320$

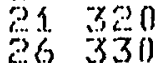

$4 \quad 320$

3.3 .5

$3 \quad 3: 0$

3320

3 320

娄 80

35
35
35
35
35

$8.9 \quad 66,1$

9.2 661

$9.7 \quad 661$

$10.0 \quad 661$

$3.3 \quad 10.3 \quad 661$

30 5. 1.1671

18 1.2. 801

14 1.1.2 701

1211.0720 10

11.2720

10.6 .74

9.8741

890

2190

2. 315

9.8

731

10.1

$50.4 \quad 70$

1.0.6 692

2310

1300

1. 320

2 315

1. 1.1

1. 1.0

4.t. 0

682

3.

68

3.1.0

663
283301909

$32 \quad 330$

313330

36270

1.310

41.300

3300

42.280

3280

40280

250

4A 330

47315

330

196 345

2315

241. 315

302315

334

998

998

300

316

$349 \quad 10$

$\begin{array}{ll}304 & 240 \\ 296 & 200 \\ 290 & 200\end{array}$

20050

150200

3
3
3
3
3

$\begin{array}{ll}95 & 200 \\ 70 & 215 \\ 50 & 215\end{array}$

49215

47 215

3240

3 200

3200

$\begin{array}{lll}1.1 .0 & 68 & 1 \\ 11.2 & 66 & 1 \\ 1.6 & 66 & 1 \\ 3.2 .1 & 67 & 1 \\ 1.6 & 67 & 0 \\ 13.0 & 69 & 0 \\ 13.8 & 70 & 0 \\ 13.6 & 71 & 0 \\ 14.2 & 72 & 0 \\ 1.4 .2 & 92 & 0\end{array}$

$\begin{array}{lll}1.4 .6 & 73 & 0 \\ 1.7 & 72 & 0 \\ 1.4 .7 & 76 & 0 \\ 1.7 & 76 & 0 \\ 13.4 & 79 & 0\end{array}$

8

1.3 .4

1.3 .1

1...9

13.7

1.2. 4

790

800

(3) 0

81. 0

8 13. 310

10 1. 1.9790

$\begin{array}{llll}13 & 1.8 & 77 & 0 \\ 13 & 3.1 .6 & 75 & 0\end{array}$

17 13.0 720

2200

3 至1

12

12.0

740

19

1.?.0

i.1. 8

1.1. 8

740

321.

18

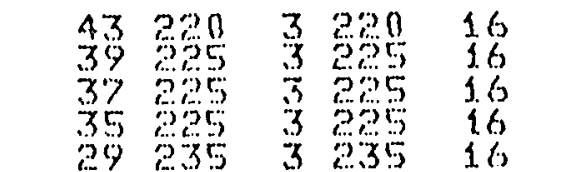

1.1. 5

1.1. 0

s. 0

3. 0.1

3. 0.4
730

728

690

690

680 
$326 \quad 57 \times 3141.0 \quad 80 \quad 22.5 \quad 23 / 4 / 80 \quad 0527$ $327586 \quad 3142.7 \quad 8028.023 / 4 / 800606$ $32859 \times 34 \quad 44.280 \quad 33.823 / 4 / 800700$ $329.60 \times 31 \quad 45.7 \quad 83039.2 \quad 23 / 4 / 80 \quad 0737$ $33031 \times 3147.48044 .6 \quad 23 / 4 / 80 \quad 0826$

331

336

334

335

336

338

339

345

34

343

344

346

347

348

350

351

35

354

$35: 3$

$3 \%$

358

$35 \%$

360

363.

360

36,3

365

366

$36 \%$

3609

370

371

$37 \frac{37}{37}$

$3 \% 4$

$3 \% 6$

378

.379

380

.3831

$38 \%$

384

38 !:

386

387

3838

389

390

620 31. $49.0 \quad 80 \quad 50.0 \quad 23 / 4 / 80 \quad 0908$ $33.3+3444.9 \quad 8050.523 / 4 / 801000$ $333+3136.28052 .523 / 4 / 803100$ $334+31.26 .88054 .523 / 4 / 801300$ $335+31$ 17.2 $8055.523 / 4 / 8030300$

$336+31 \quad 7.59056 .523 / 4 / 80 \quad 1400$ $337+30 \quad 58.7 \quad 8057.223 / 4 / 803500$ $338 \times 3049.98059 .1 \quad 23 / 4 / 801600$ $339+3041.281$ 1.2 $23 / 4 / 803700$ $340+3032.4$ B1 $2.923 / 4 / 801800$

$341+30 \cdot 23 ; 681$

$340+30 \% .88$ 81.

$343+30.5 .983$

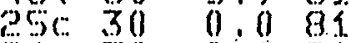
$24 \times 30$

0.0 .83 .

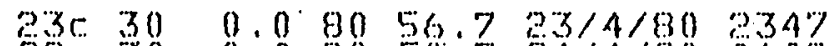
$52 \times 30 \quad 0.08050 \% 754 / 4 / 800100$ 216 30 0.0.80 45.0 $34 / 4 / 80$ 014A $10 \times 30$

0.0 .80 - $0004 / 4 / 800304$ 19030

$0.080 \quad 33.0 \quad 24 / 4 / 80 \quad 0344$

$3.8 \times 30 \cdots 0.38026 .924 / 4 / 80 \quad 0536$ 17630 $1.6 \times 30$ 1.5030

0.080 26. $34 / 4 / 80$ 0 549 $0.28038 .354 / 4 / 800 \% 04$ 1. $4 x .30$ $\begin{array}{lllll}0.0 & 80 & 15.8 & 24 / 4 / 80 & 0720 \\ 0.0 & 30 & 33.0 & 54 / 4 / 830 & 0347\end{array}$

$13 \mathrm{C} \quad 30 \quad 0.0 \quad 80 \quad 10.504 / 4 / 80 \quad 0907$ $35 \% 730$ 2.5 830 1\%.2 $4 / A / 80.3 .00$ $358 \% 595 \%, 0$ 80 $1 \%, 524 / 4 / 801200$ $359+29.56 .5 \quad 3017.824 / 4 / 80$ 1.310 $360+2950.080$ 19.4 $24 / 4 / 80 \quad 3300$

$361+2950.18019 .024 / 4 / 801315$ $36 \%+2950.0$ 80 $17.620 / 4 / 80 \quad 3330$ $363 \times$ 59 $50.5801 .3 .524 / 4 / 801400$ $364 \times 3050.780$ 6.0 $54 / 4 / 801436$ $365 \times 2850.58010 .524 / 4 / 80$ 1.51:2

$366 \times 29 \quad 50.4 \quad 8014.924 / 4 / 80 \quad 1538$

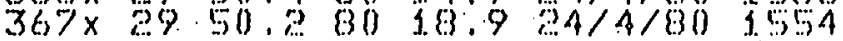
$368 \times 2950.200$ 21. $354 / 4 / 801614$ $369 \times 59.50 .08023 .5944 / 801604$ $370 \times 2850.280 \quad 26.234 / 4 / 801638$

$373 x .2950 .28028 .324 / 4 / 80 \quad 1755$ $370 \times 2950.280-31,104 / 4 / 80$ 1741 $373 \times 2080.28083 .204 / 4 / 80 \quad 3 \% 53$

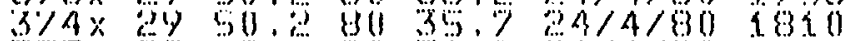

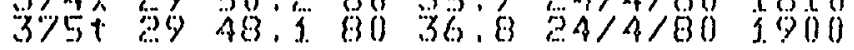

$376+2930.18039 .204 / 4 / 8020000$ $377+2930.580 .42 .054 / 4 / 806100$ $378+2922.0 \quad 8044.524 / 4 / 802500$ $379+5937.96047 .424 / 4 / 803300$

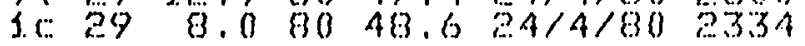

$3 \times 299.0 \quad 80 \quad 43.0 \quad 25 / 4 / 80 \quad 0034$

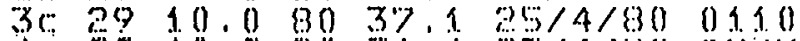
$4 \times 29 \quad 10.8 \quad 8031.4 \quad 25 / 4 / 80003 \%$ $562943.0 \quad 80$ 25.7 $25 / 4 / 800313$ $6 \times 29 \quad 13.0 \quad 80$ 20.3 $25 / 4 / 80 \quad 0433$

$76.29 \quad 14.1 \quad 80$ 1. 96. $29 \quad 14.9 \quad 80$ $10 \times 3935,380$ 1.te 15.880

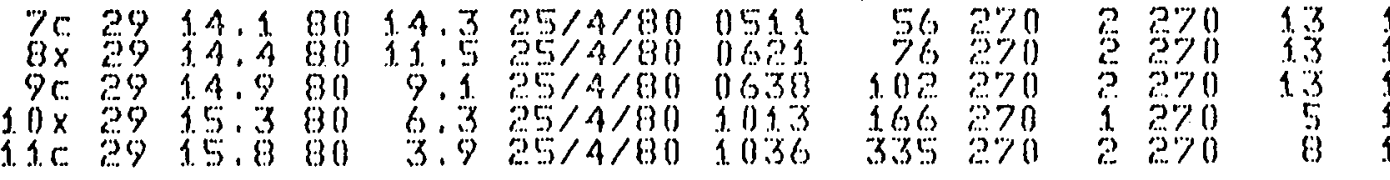

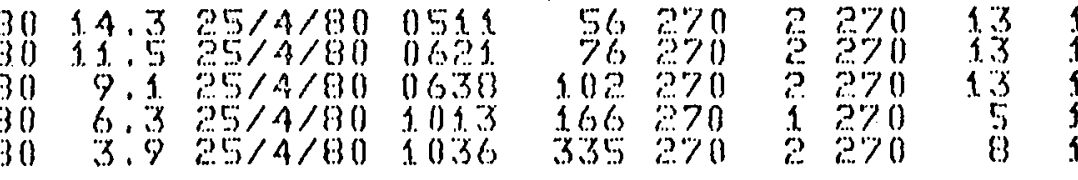

$4.353 / 4 / 8301900$

$6.023 / 4 / 8020000$

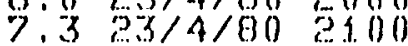

$8.7 \quad 33 / 4 / 80 \quad 0145$

2.. $53 / 4 / 80 \quad 2305$

$\begin{array}{ll}30 & 235 \\ 24 & 535 \\ 2 & 2.35\end{array}$

$18 \quad 215$

16. 245

$13 \quad 245$

3.2 260

13260

$16 \begin{array}{ll}31.5 \\ 16\end{array}$

20200

20200

56300

20380

26190

20

15210

21530

25250

34230

36 , 30

$40 \quad 230$

430

$45 \quad 230$

(4) 240

76240

202.340

$290 \quad 250$

316
9040
949

990040

49215

50245

$50 \cdot 215$

909015

420 อ15

$330 \quad 21.5$

1. 0329

54 - 30

47210

47210

44 에이

39) 210

$38 \quad 210$

36210

34210

3.1. 150

?7 210

34 行

2320

30 270

20 $2 \% 0$

ว

(2)

32.270

$37 \quad 370$

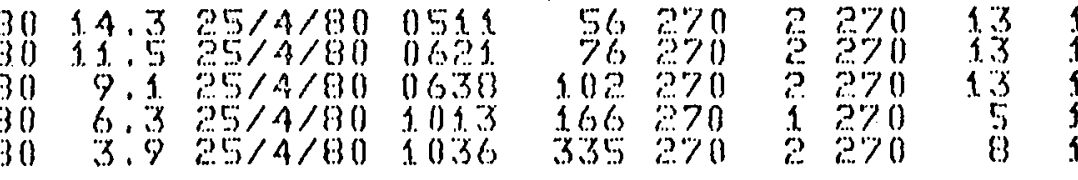

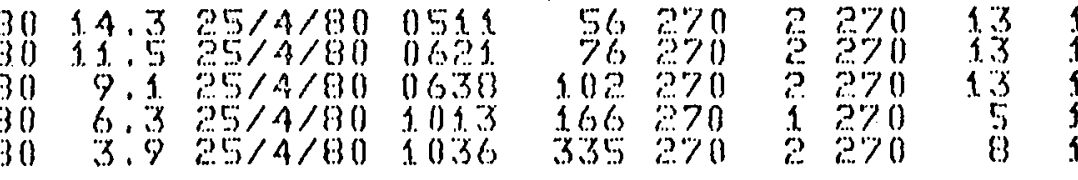

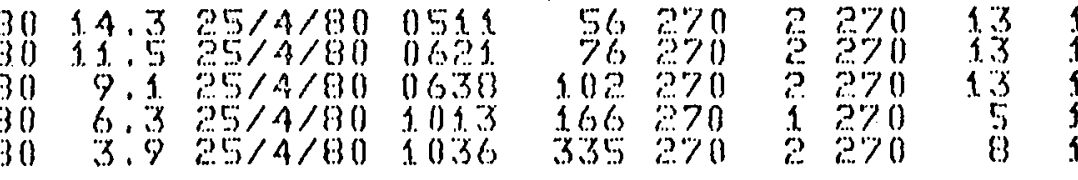

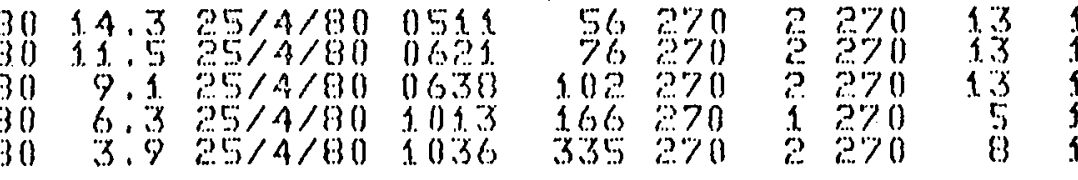

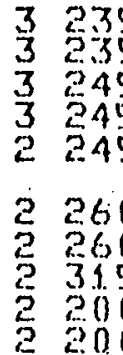

16

$10.6 \quad 670$

16

3.6

11.

670

11.2 66

$11.0 \quad 680$

10

11.1790

10 11.2 730

3.1 3.1.6 740

2 20

110

$\begin{array}{ll}74 & 0 \\ 74 & 0\end{array}$

$\begin{array}{lll}2 & 200 & 10 \\ 2 & 1830 & 12 \\ 2 & 1.90 & 12 \\ 2 & 1.80 & 12 \\ 2 & 1.80 & 15\end{array}$

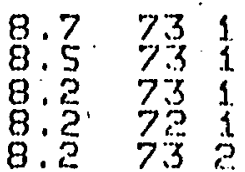

2. 180

13

2. 200

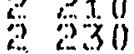

1.8

14

$2 \quad 230$

2530

2300

in

$8 \cdot 2$

9.8

1.0.1

1.0. 0

745

$10.0 \quad 701$

1.0.0 603

8.7. 681

8.5 .68 .4

8.568 .1

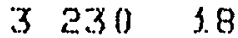

8:9 68 1

3.230

3.

20

20

$9.0 \quad 68 \quad 1$

$9.6 \% 5$

$9.7 \quad 734$

\section{3 arjo}

?0

3540

3240

20

.1.0

$\begin{array}{lll}3 & 2 & 1 \\ 3 & 2 & 15 \\ 3 & 2 & 15\end{array}$

3 北

10

10

50

3 2...

3. (1)

1. 8.2

9.9

74

9.576

9.176.

3215

10

3 की

3 în

(7),

763

8.9

8.6

8. . .

79

8.079

$9.0 \quad 791$

18

3 \%1. 10

7.976

6.0 78 a

$\begin{array}{llll}3 & 210 & 18\end{array}$

7.9

764

7.6774

3510

$3 \quad 10$

18

7.6

$7.6 \quad 7.84$

$7: 6$ 73: 4

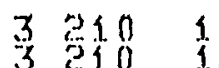

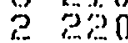

250

2. 270

1. 11

9.

73

.1701

$50.6 \quad 701$

$10.6 \% 01$

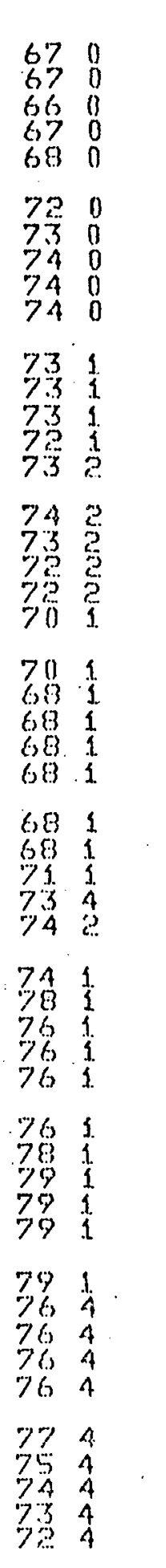


page 7

391. $391+2925.8 \quad 80 \quad 5.0 \quad 25 / 4 / 80 \quad 1300$

392 392 2932.680 5.0 $25 / 4 / 801330$

$393393+2939.780$

$394 \cdot 394+2946.280$

$395.395+2953.080$

$4.525 / 4 / 801400$

$3.525 / 4 / 80 \quad 1.430$

$2.525 / 4 / 80 \quad 1500$

396
397
396
395
400

401

$40:$

$40 \div 3$

404

405

406

408

$40 \%$

410

41.

41.2

A1.

$41 \mathrm{~s}$

41.6

$41 \%$

41.

420

431

42

424

425

426

$4 \%$

48

430

431

434

435

436

$43 \%$

$0.0 \quad 80$

4. $525 / 4 / 801530$

$396+30$

$397 \mathrm{C} 30 \quad 1.480$

$398 \times 30$

2.4. 80

$2.855 / 4 / 80 \quad 1559$

$13030 \quad 0.280$ 10.4 $55 / 4 / 801824$ $400+2958.060$ 12.5 $25 / 4 / 80$ 20.00

$401 \times 2955.0 \quad 8013.0 \quad 25 / 4 / 80.2125$

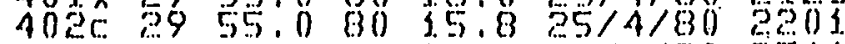

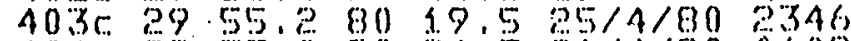
$404 C: 395 \%, 0 \quad 8004.526 / 4 / 8001.09$ $405 \times 29.55 .0 \quad 8028.026 / 4 / 80 \cdot 0230$

$406 \times 2955.08030 .020 / 4 / 80.0245$ $407 \% 29.55 .0 .803 \%, 0 \quad 36 / 4 / 800335$ $408+5956.0 \quad 8037.056 / 4 / 8005000$ $409+30=4.28036 .836 / 4 / 800600$ $410 t 30$ 1.2. 80 28.926/4/80 0700

$411+3020.38021 .856 / 4 / 800800$ $412+3053.3 \quad 80 \quad 17,026 / 4 / 800830$ $413+3026.5801 \% .206 / 4 / 800900$ $414 \mathrm{r} 3030.580 \quad 7.756 / 4 / 800930$ $4.5+30 \quad 34.580 \quad 2.826 / 4 / 801000$

$416+3039.9 .9957 .5 \quad 26 / 4 / 801030$ $417 \% 30$ 40.5 $7956.7 \quad 26 / 4 / 80$ 3040 $418 \times 3040.980 \quad 1.526 / 4 / 801317$ $419 \times 3041,5804.056 / 4 / 80 \quad 1334$ 42063041,80

$3.026 / 4 / 80$ 1.496

42)1: 3044.880

$42 \times 3 \times 304580$

40363043.480

$6.4 \quad 26 / 4 / 80 \quad 1615$

6. $36 / 4 / 80$ 3. 35

$13.626 / 4 / 80 \quad 1802$

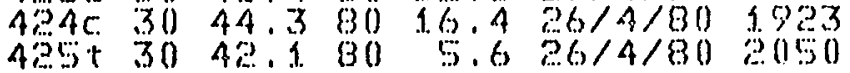

$426 \times 3046.580$

$427 \times 3051.580$

$420 \times 30 \quad 56.4 \quad 80$

$4296 \quad 3056.680$

4. $026 / 4 / 80$ 2.

2. $06 / 4 / 80$ ?

$0.426 / 1 / 80$ 250

1.4 $26 / 4 / 80$ ?237 $430 \mathrm{C} 3$

$430 \times 3055.57956 .557 / 4 / 800104$ $432 \mathrm{c}$
4330
434 $434 t$

$435+3$

$436+3120.97937 .527 / 4 / 80 \quad 0415$ $7.07949 .0374 / 80$ 0345 10:0 $7945: 3 \quad 27 / 4 / 80$ 03:5

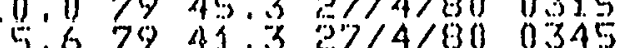
$437+31.26 .5793 .627 / 4 / 80 \quad 0445$
999270

999570

999270

999270

$999 \quad 270$

9992770

$430 \quad 1 \% 0$

$368 \quad 1 \% 0$

290170

$212 \quad 195$

47 175

$43 \quad 1,80$

40180

$38 \quad 1.80$

3.7. 180

263.95

$35 \quad 195$

36195

$40 \quad 185$

42185

66185

1921.85

225185

287210

$305 \quad 210$

$190 \quad 350$

$3.08 \quad 210$

1.90 2.30

$\begin{array}{ll}61.50 \\ 48 & 1 \% 5\end{array}$

$451.6 \%$

40200

67290

50285

57520

$48 \quad 180$

3.58 1.80

$154 \quad 180$

$206 \quad 3.80$

$090 \quad 180$

23250

269210

272 ?10 $\begin{array}{ll}1.270 \\ 1 & 270 \\ 1 & 270 \\ 1 & 270 \\ 1 & 270\end{array}$

$\begin{array}{llll}5 & 13.11 & 78 & 0 \\ 5 & 19.9 & 82 & 0 \\ 5 & 19.6 & 82 & 0 \\ 5 & 12.1 & 84 & 0 \\ 5 & 1.9 & 84 & 0\end{array}$

$\begin{array}{ll}1 & 270 \\ 2 & 170\end{array}$

$3 \quad 170$

$\begin{array}{lll}3 & 1.70 \\ 2 & 175\end{array}$

$\begin{array}{ll}3 & 195 \\ 3 & 175\end{array}$

3175

3 .

3180

$3 \quad 180$

$3 \quad 180$

33.95

3195

$\begin{array}{ll}3 & 185 \\ 3 & 189\end{array}$

$\begin{array}{ll}3 & 1.85 \\ 3 & 1.85\end{array}$

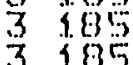

3210

3 हi.

3 2.

3 in

3210

$\begin{array}{rrrr}5 & 11.9 & 84 & 0 \\ 5 & 11.6 & 82 & 0 \\ 13 & 11.2 & 76 & 0 \\ 10 & 11.3 . & 76 & 1 \\ 7 & 11.6 . & 74 & 1\end{array}$

18 1.7.78 74

1911.61731

20 1. 11.741

$2010.5 \% 741$

$18 \quad 10.5 \quad 743$

$15 \quad 10.9 \quad 731$

18 1.7.70

30150

3165

3 . 390

3290

is

18

1.5

12.3

1.6 .6

1:. 7

740

14.13 .2750

$14 \quad 13.6 \quad 750$

13 13.7 760

$12 \quad 12.6 \quad 763$

10 12.5 761

3 : 85

3585

$\begin{array}{ll}3 & 1000\end{array}$

2380

1. 8

14

15

1.2. 3

761

1. 1...

1.3.

74

11.9705

3580

3.80

35.80

3 2.t.

3 .

10

11.8700

12

1.1.

1.1.8

10 1.1.0 7.

$?$

71.2

$15 \quad 90.5 \quad 742$

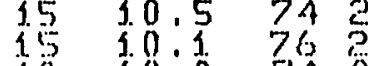

10 10.0 74

$\begin{array}{llllll}3 & 210 & 10 & 9.9 & 74 & 1 .\end{array}$

3 缃 10

$9.8 \quad 741$ 


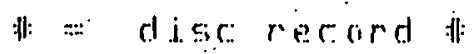

station $=$ assigned stotion number

L.atitude and bongj.jude:

Dnte and time in enstern standard time?

ty. $=$ water depth in meters

Wuld $=$. wrue direction

SS.:- SFA STATE

WND $=$ wind diraction

w) $=$ Wind Spes

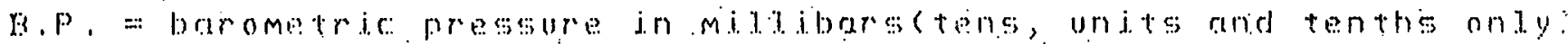
Ar $=$ a.jeremp in Fot very good \#):

W :-: Wrot wenther. code

9998 ind icate no rata 
$\begin{array}{llll}82 & 81 & 80 & 79\end{array}$

圈

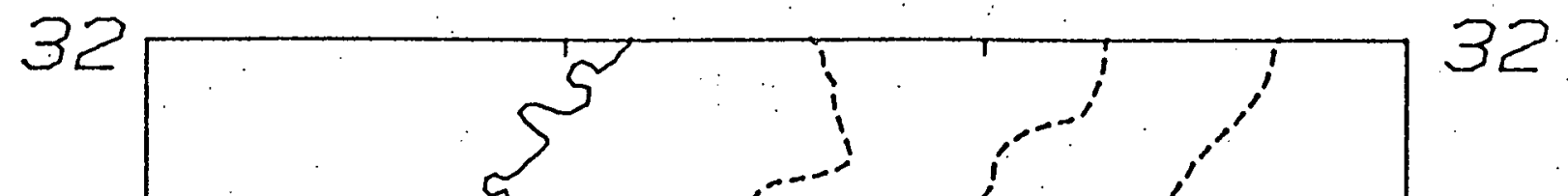

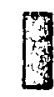

is

31

$3 \varnothing$

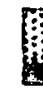

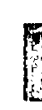

29

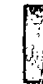

示

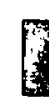

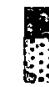

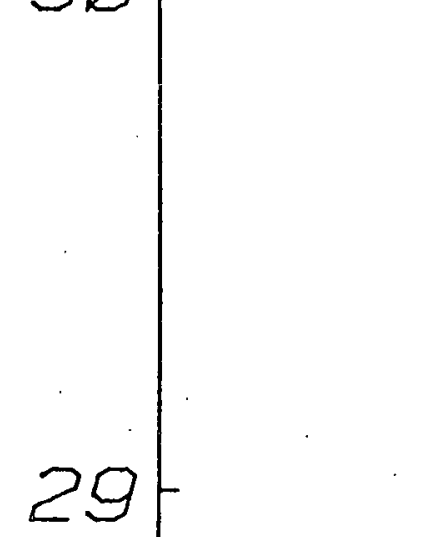

8
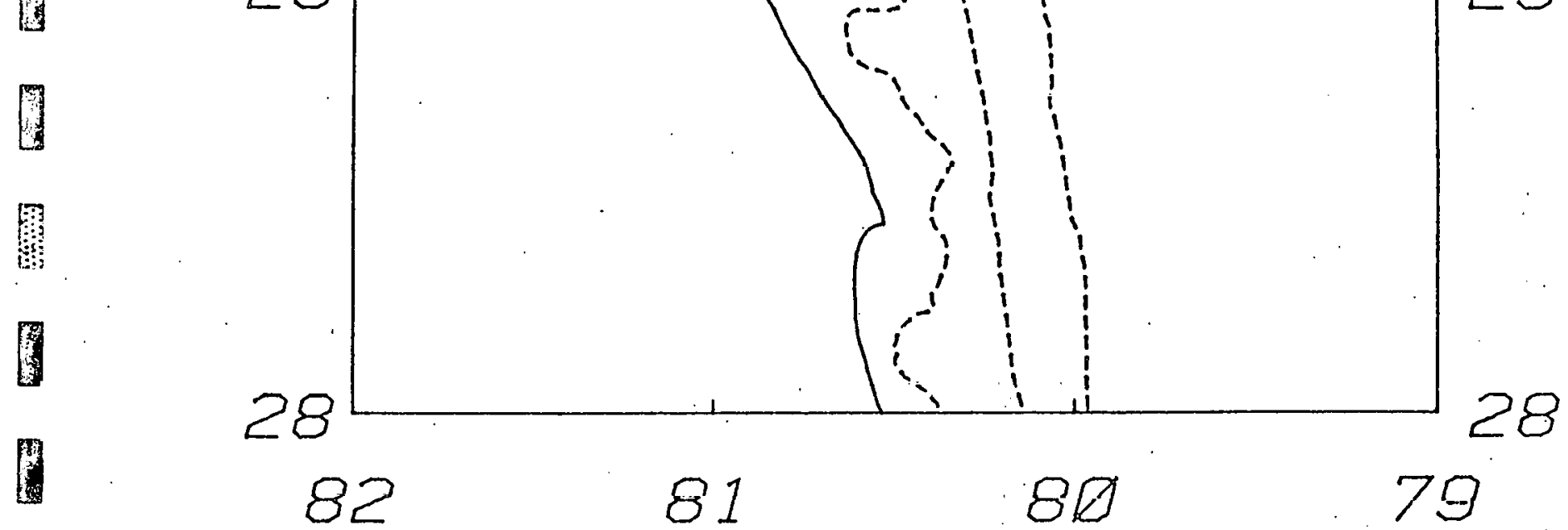


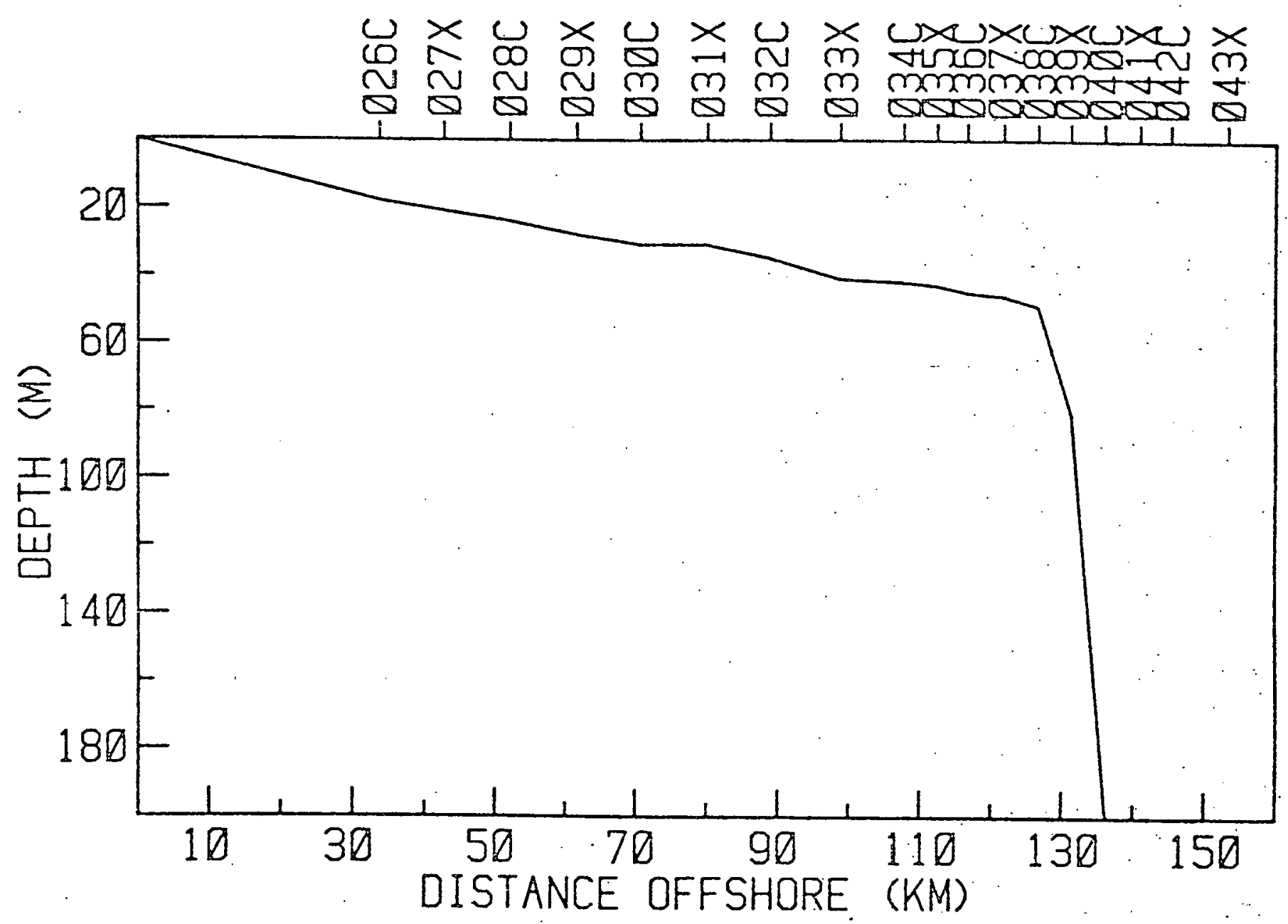




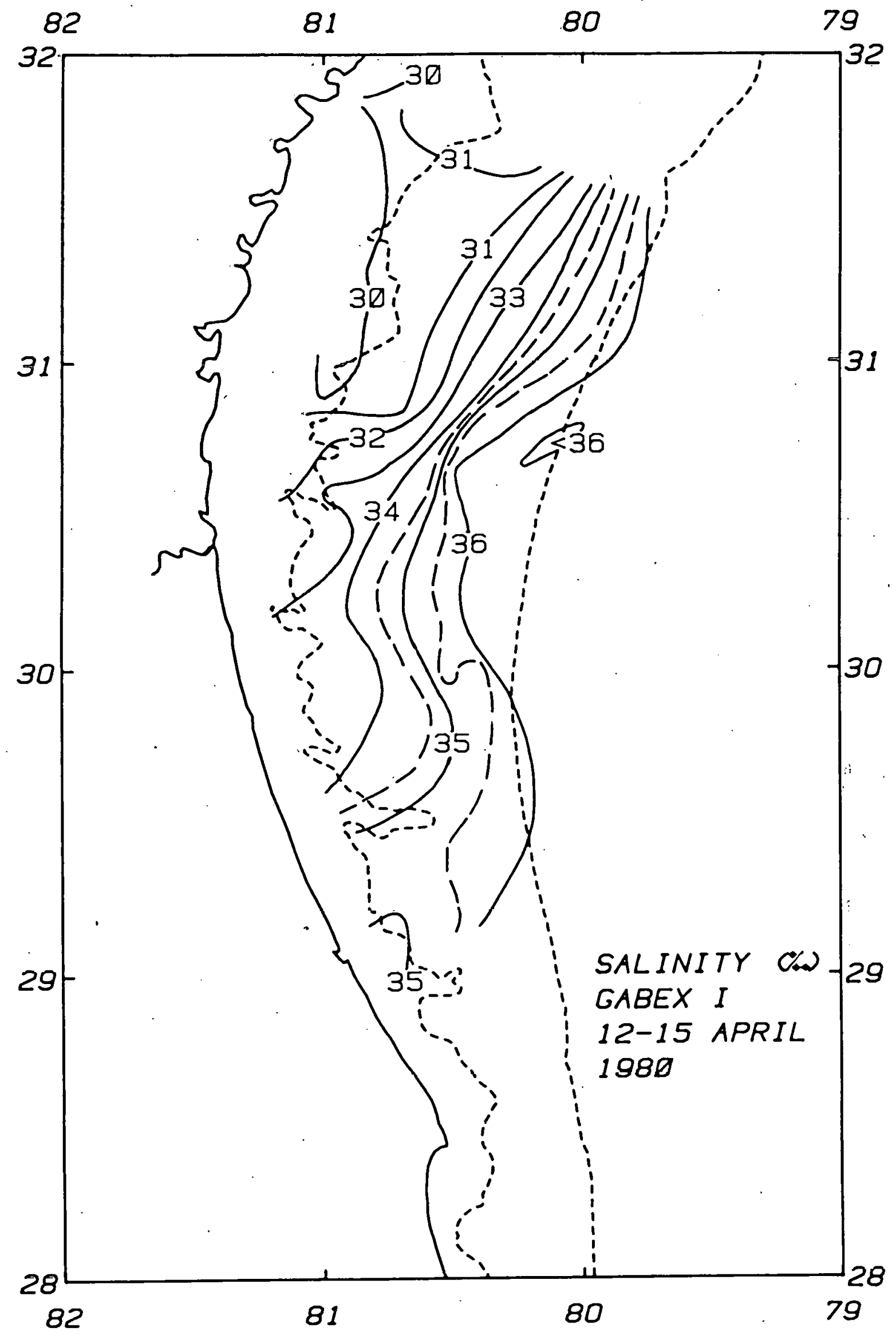




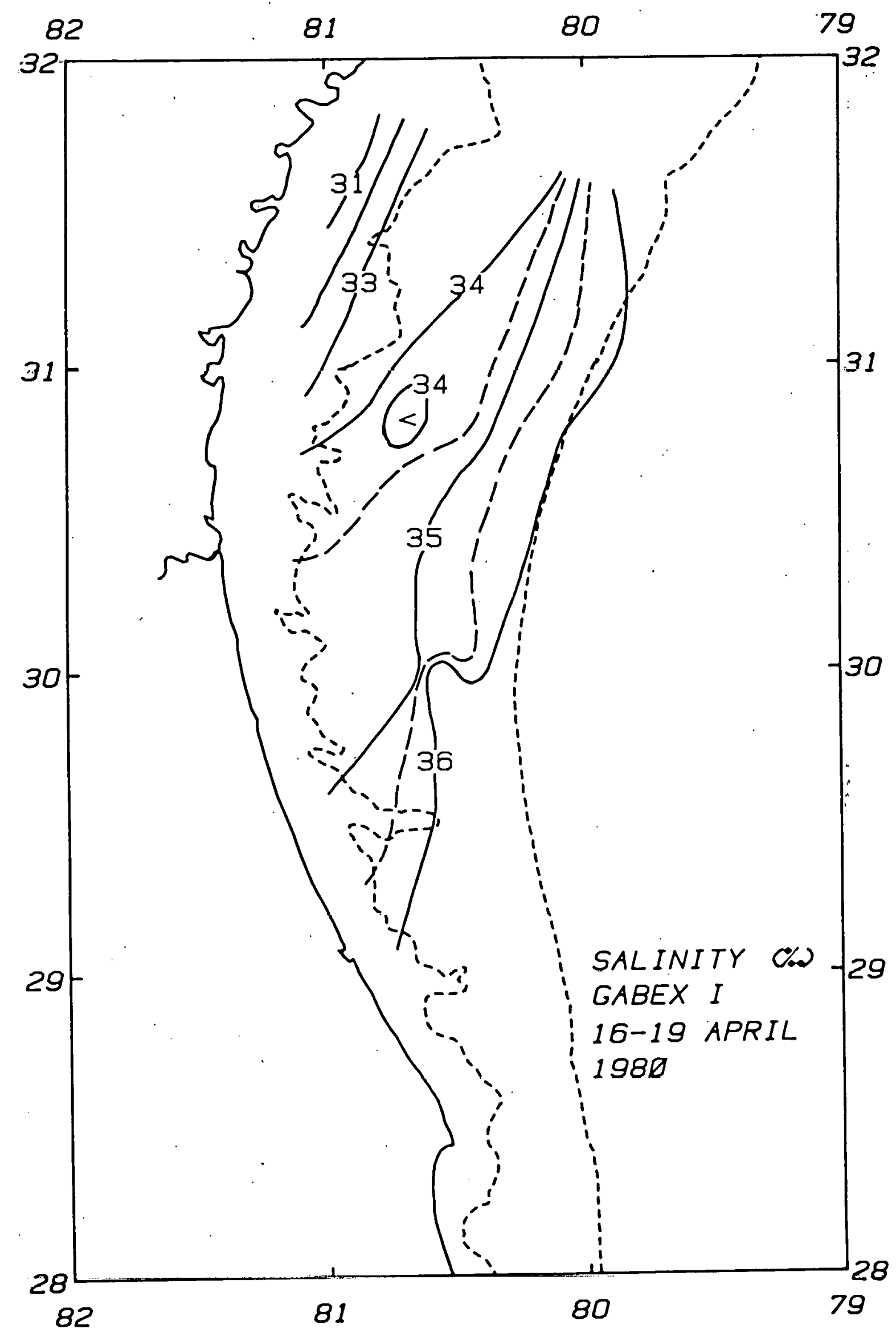




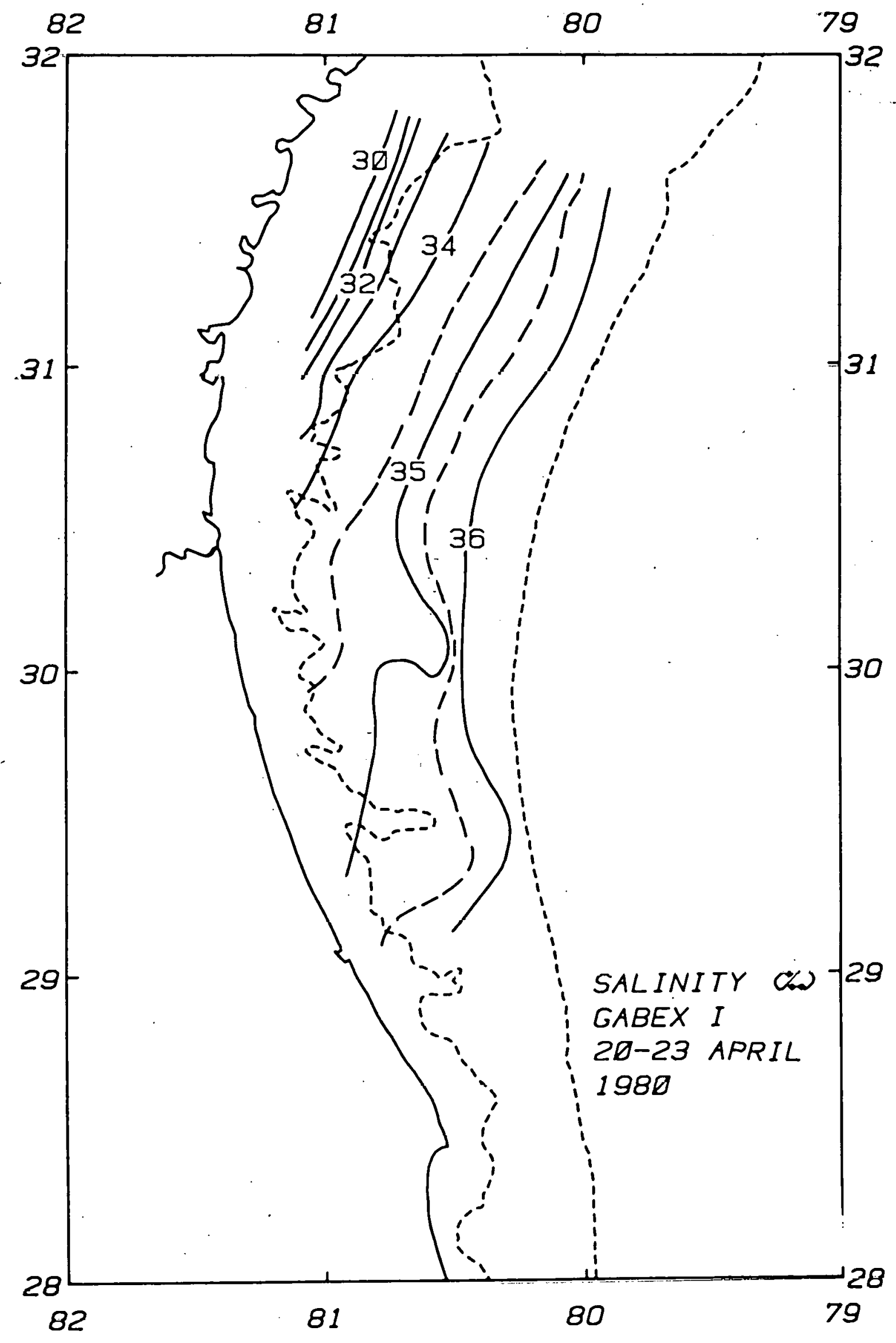




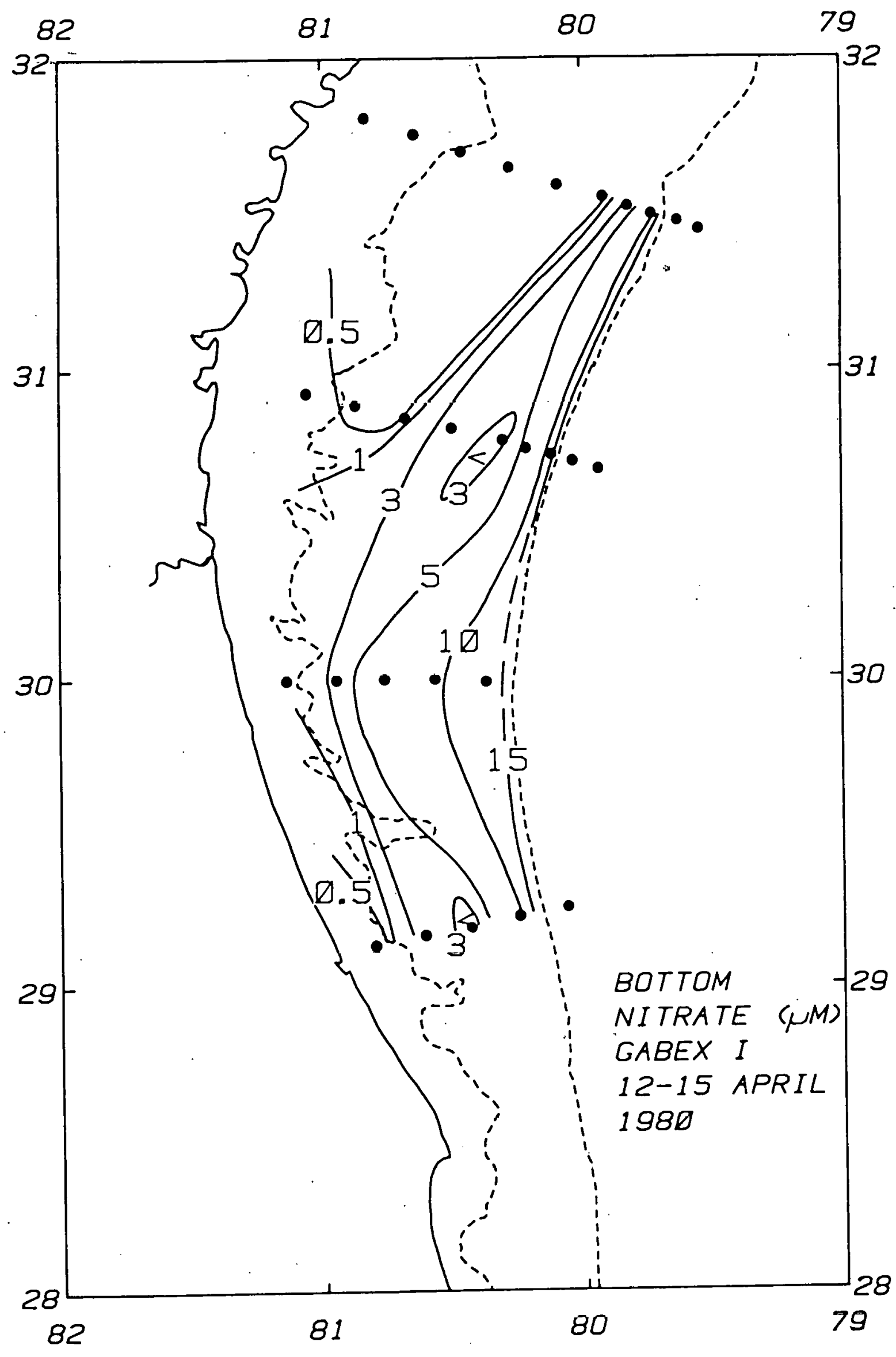




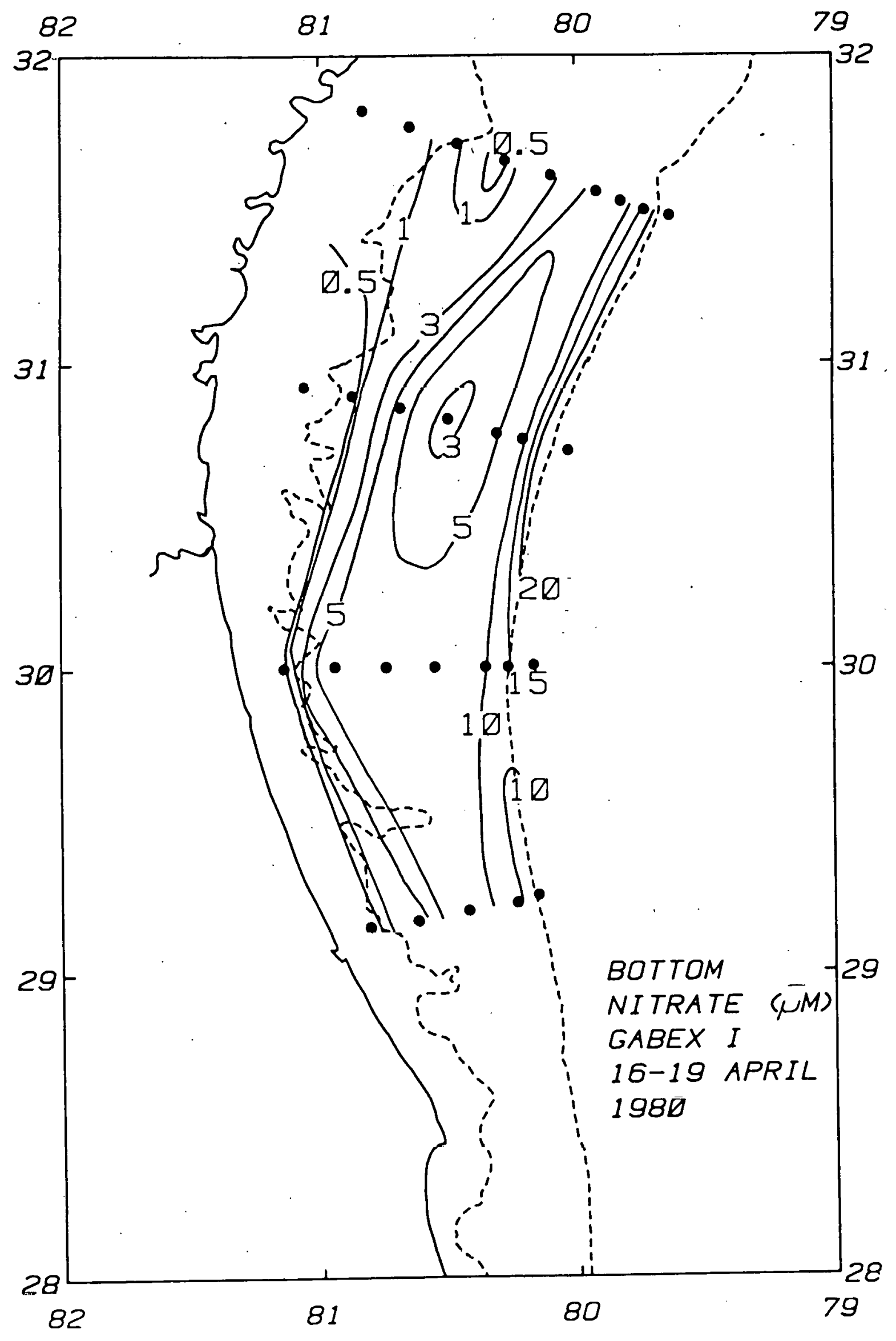




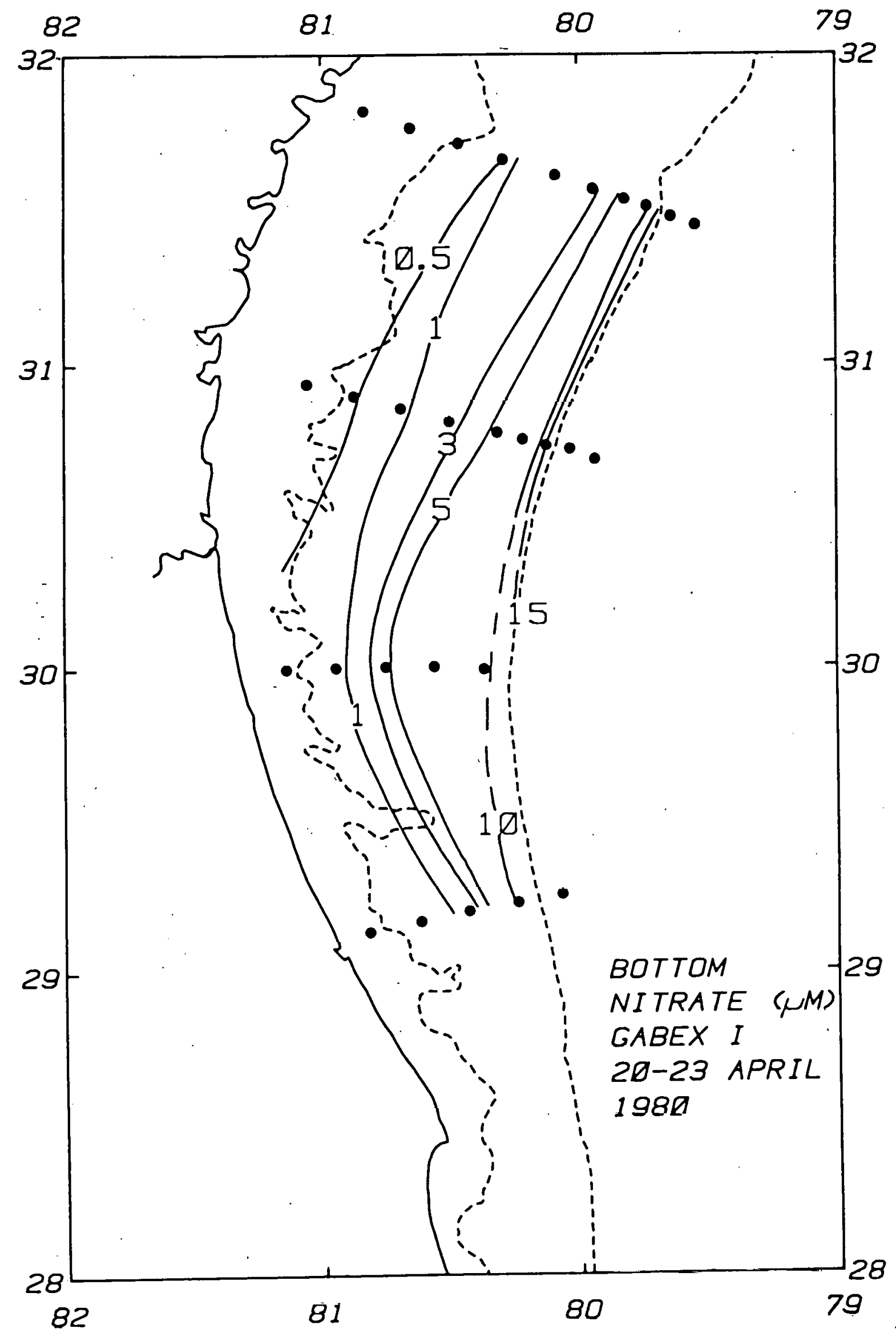




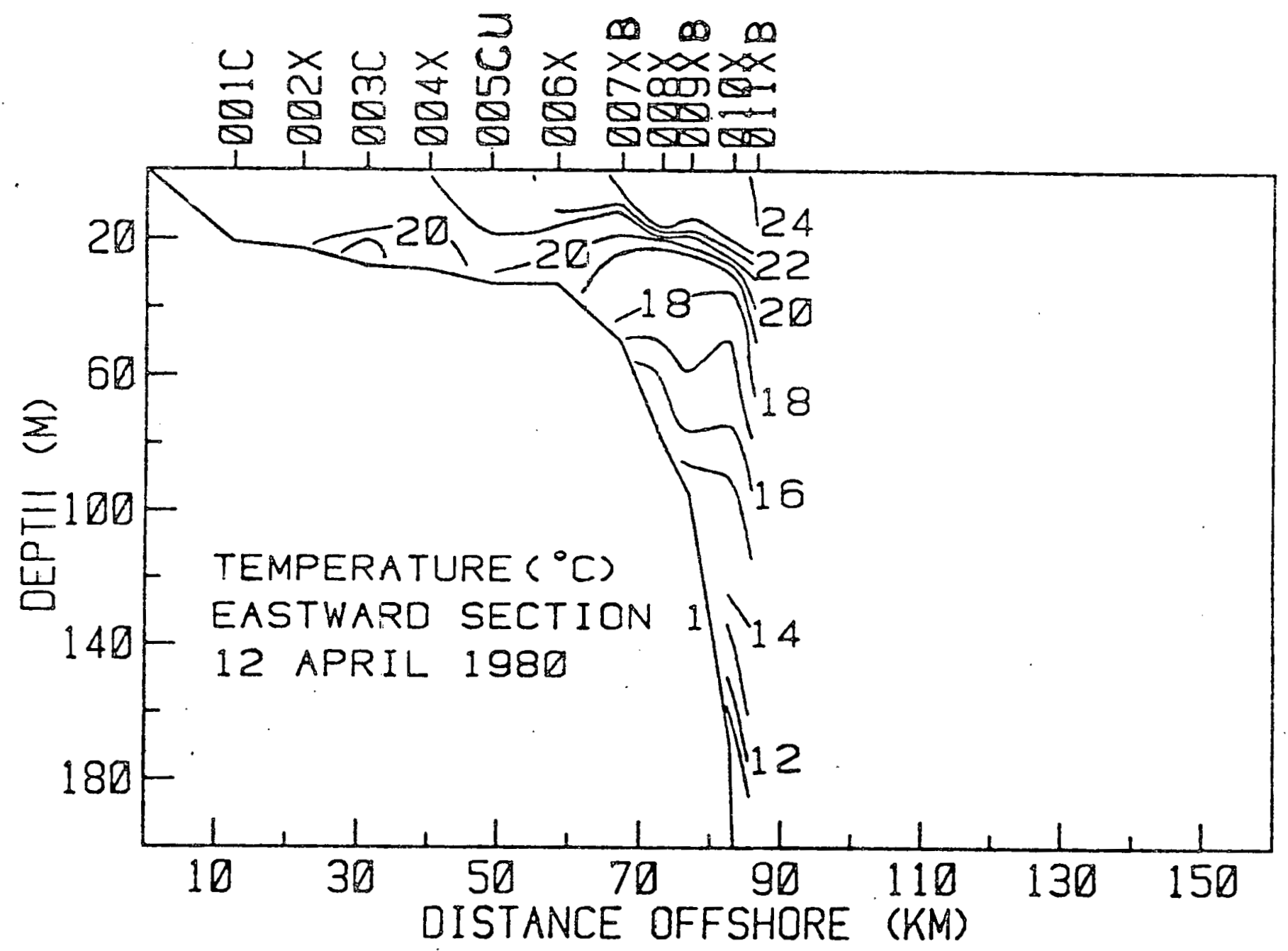




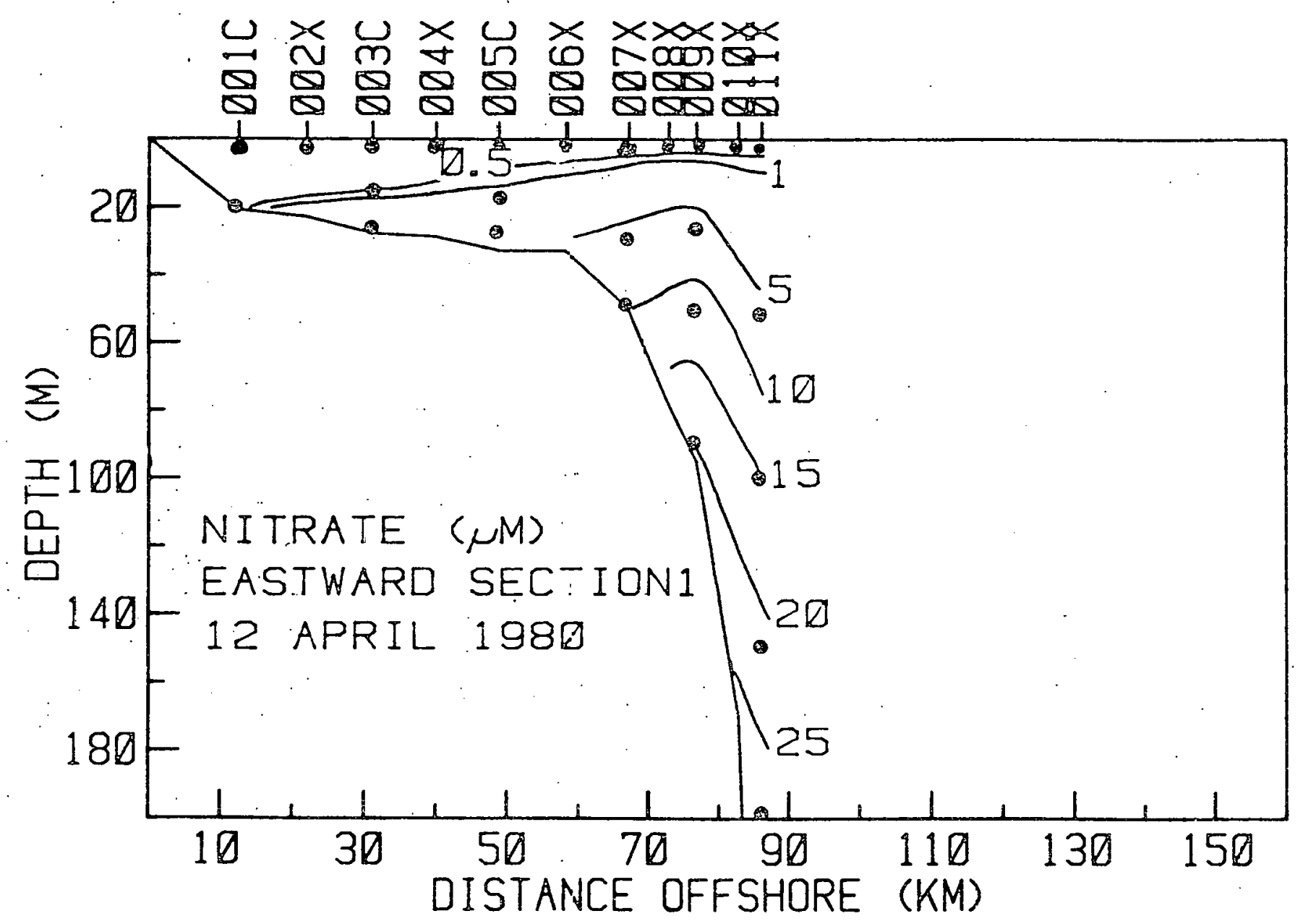




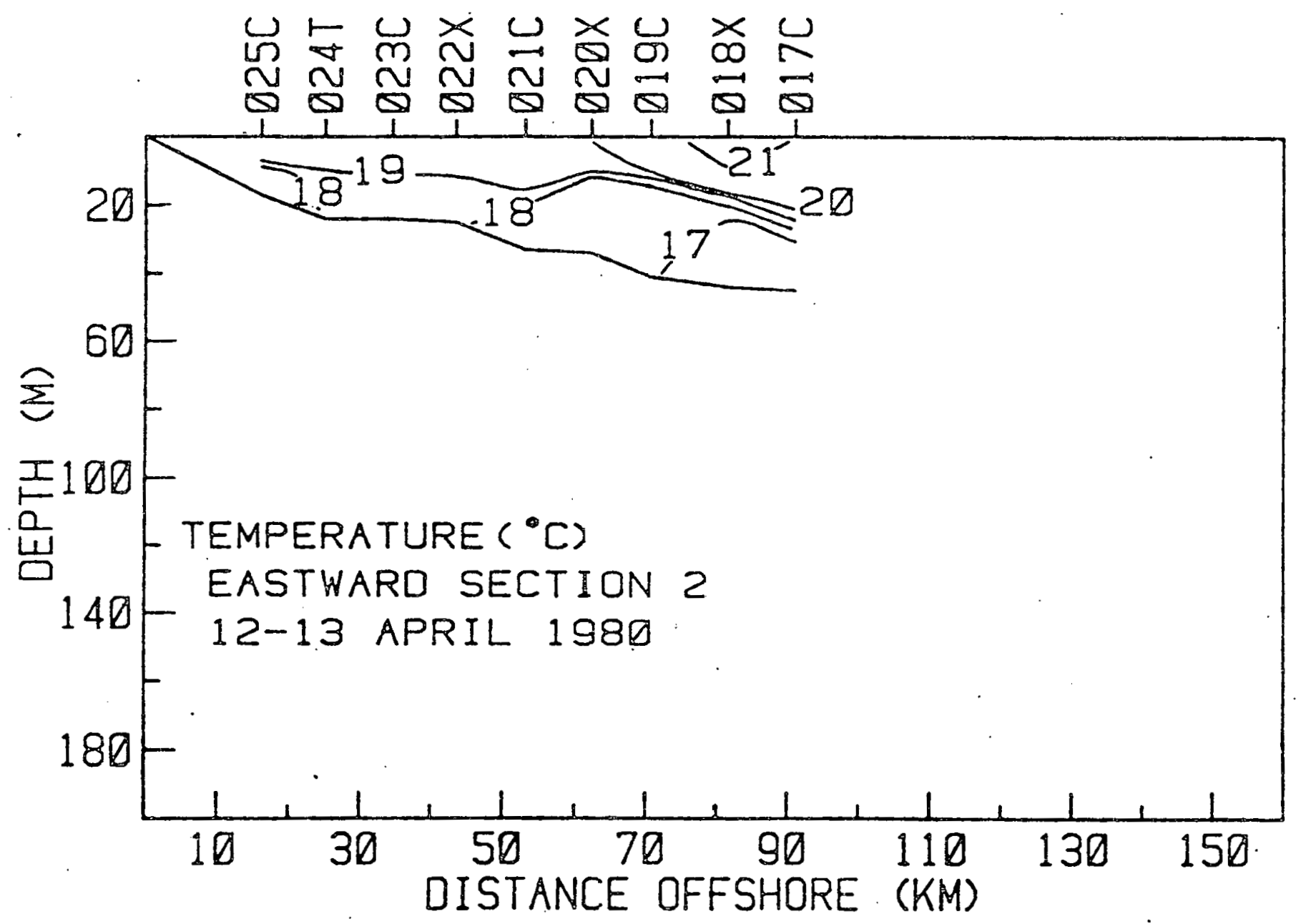




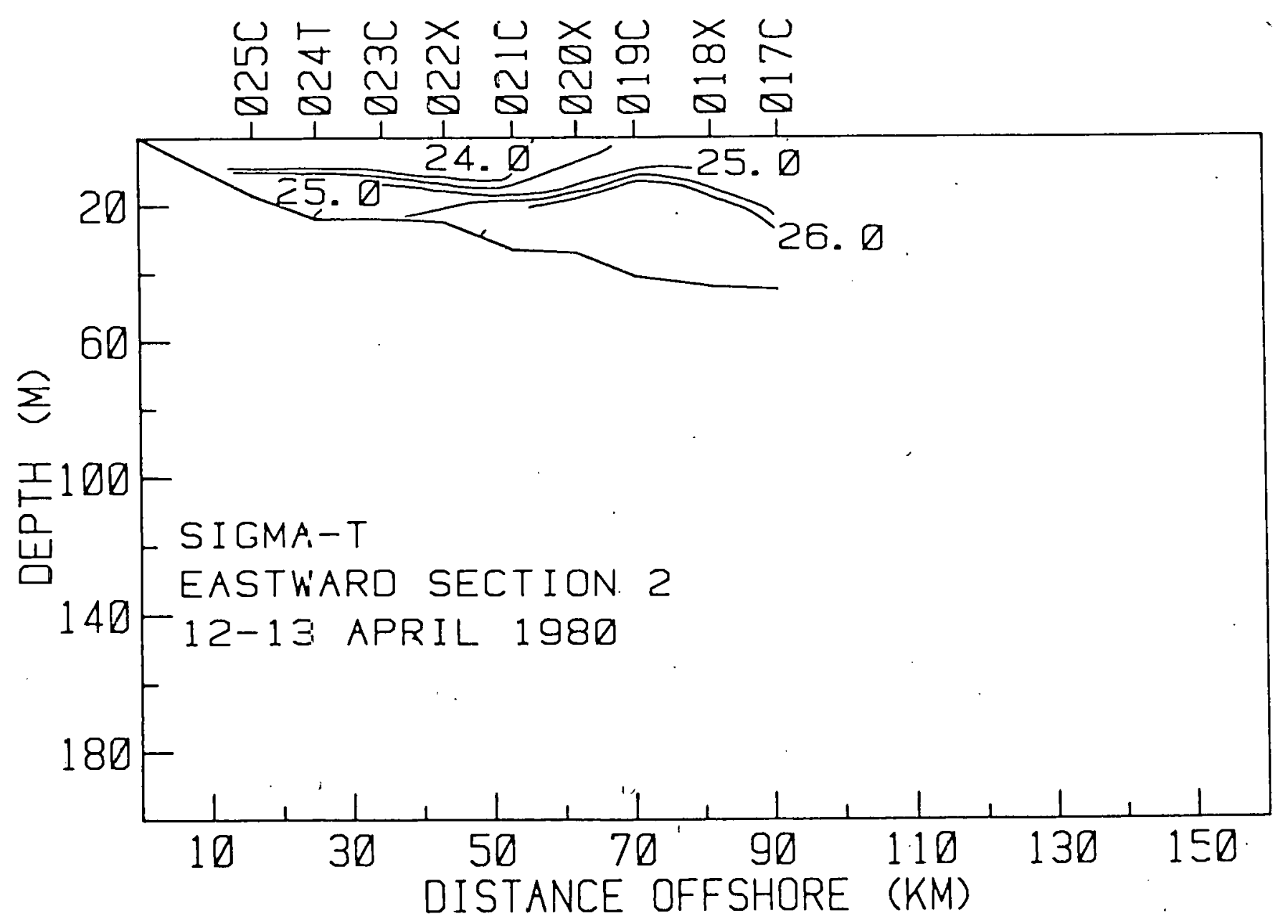




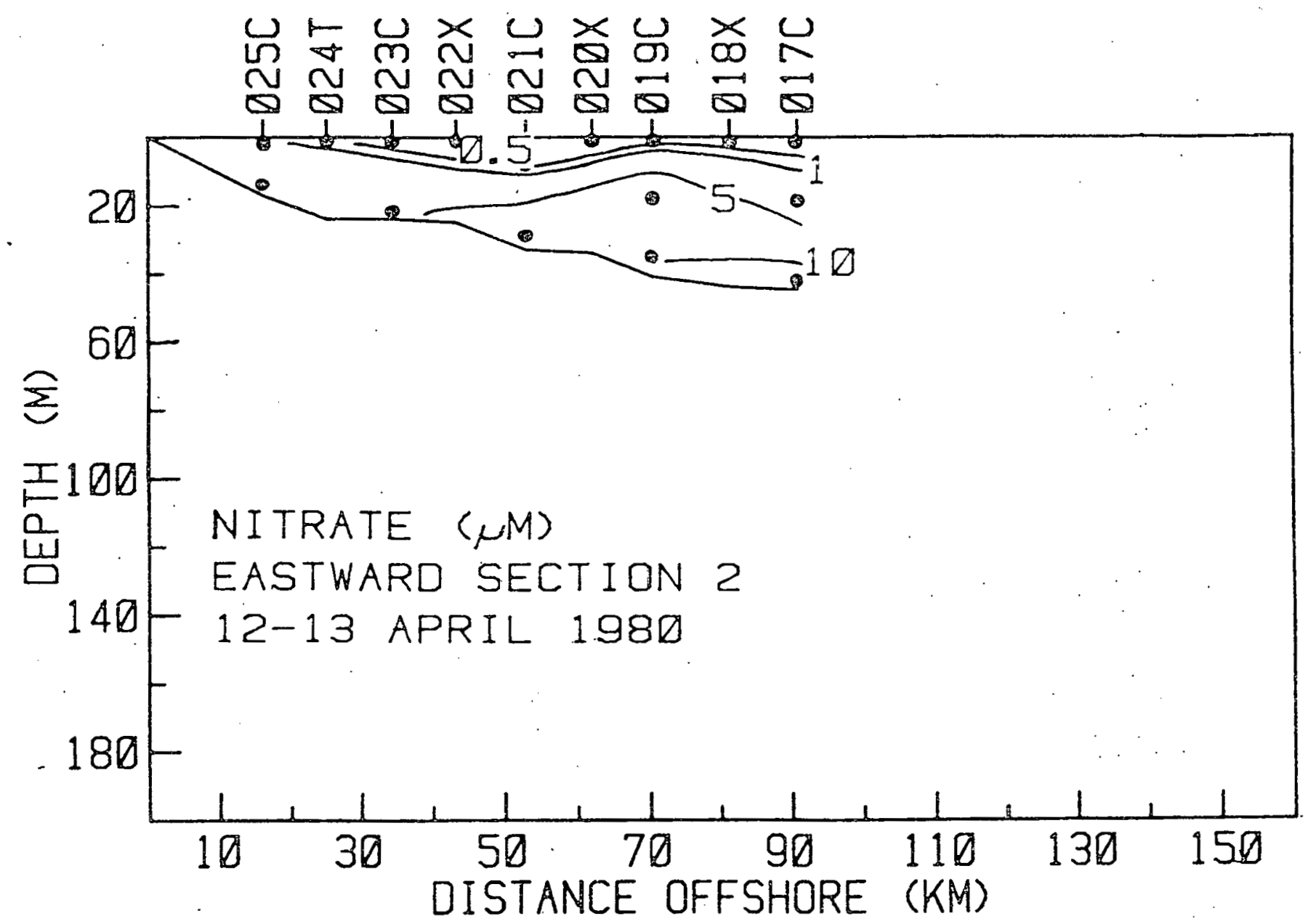




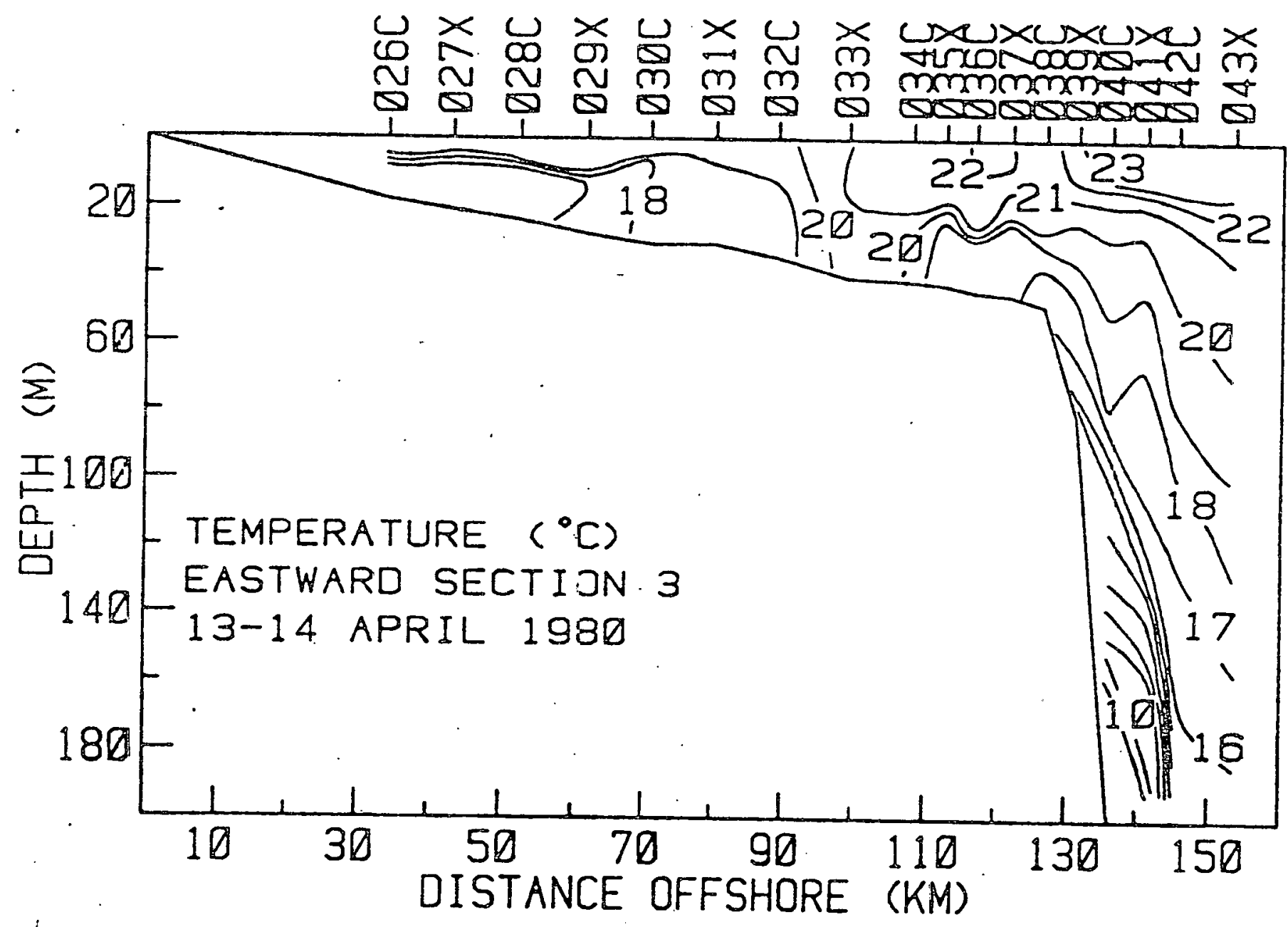




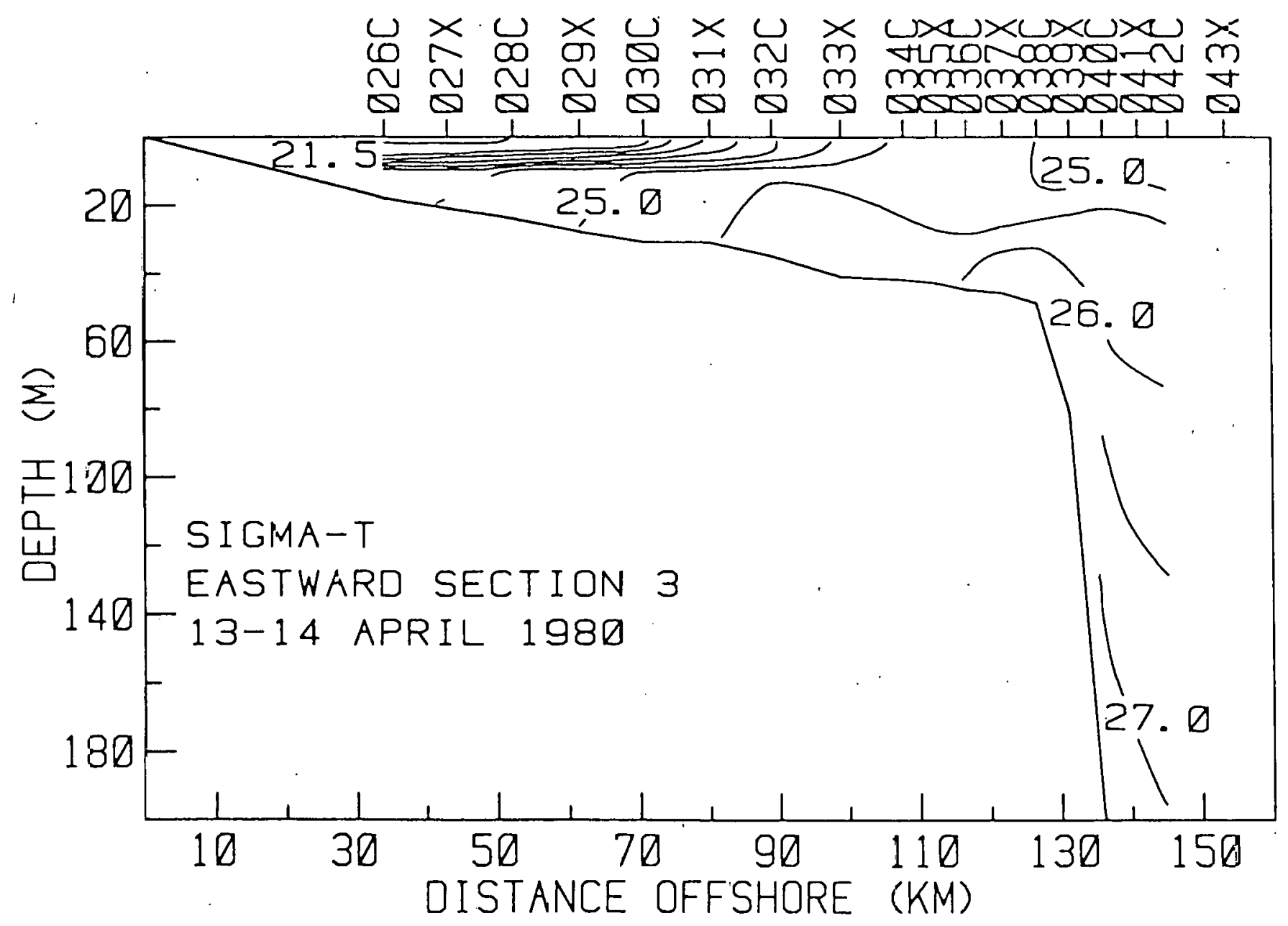




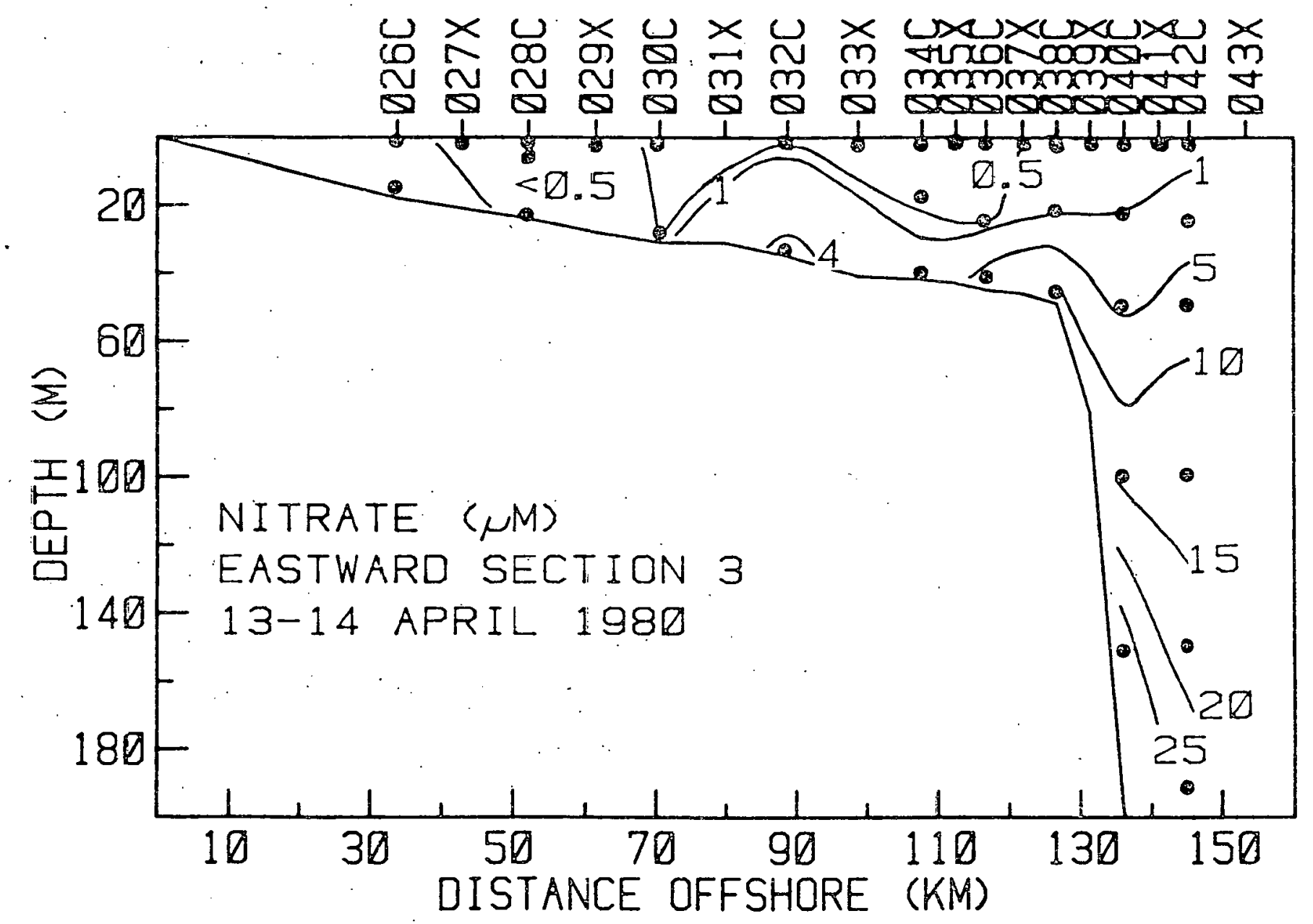




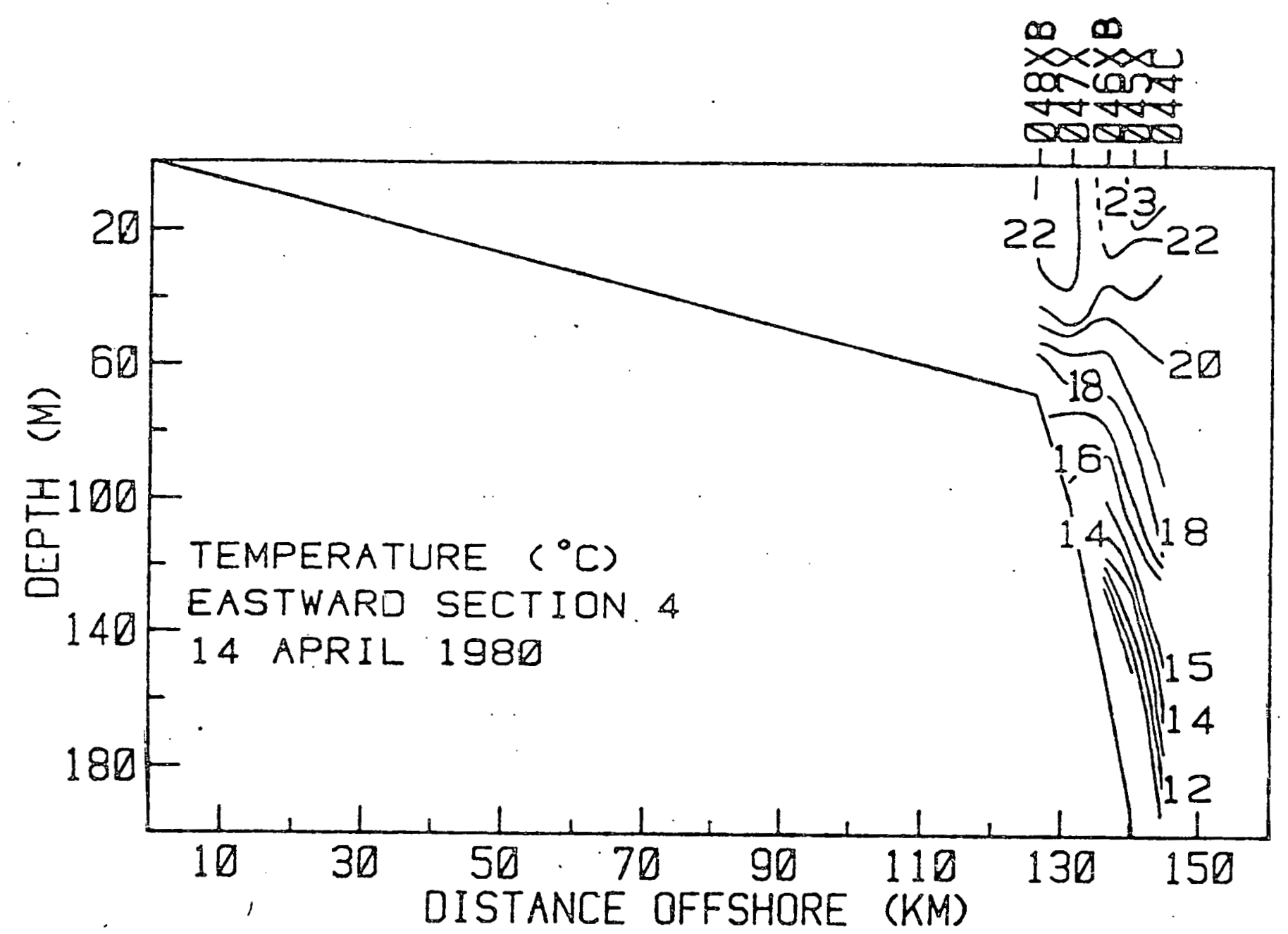




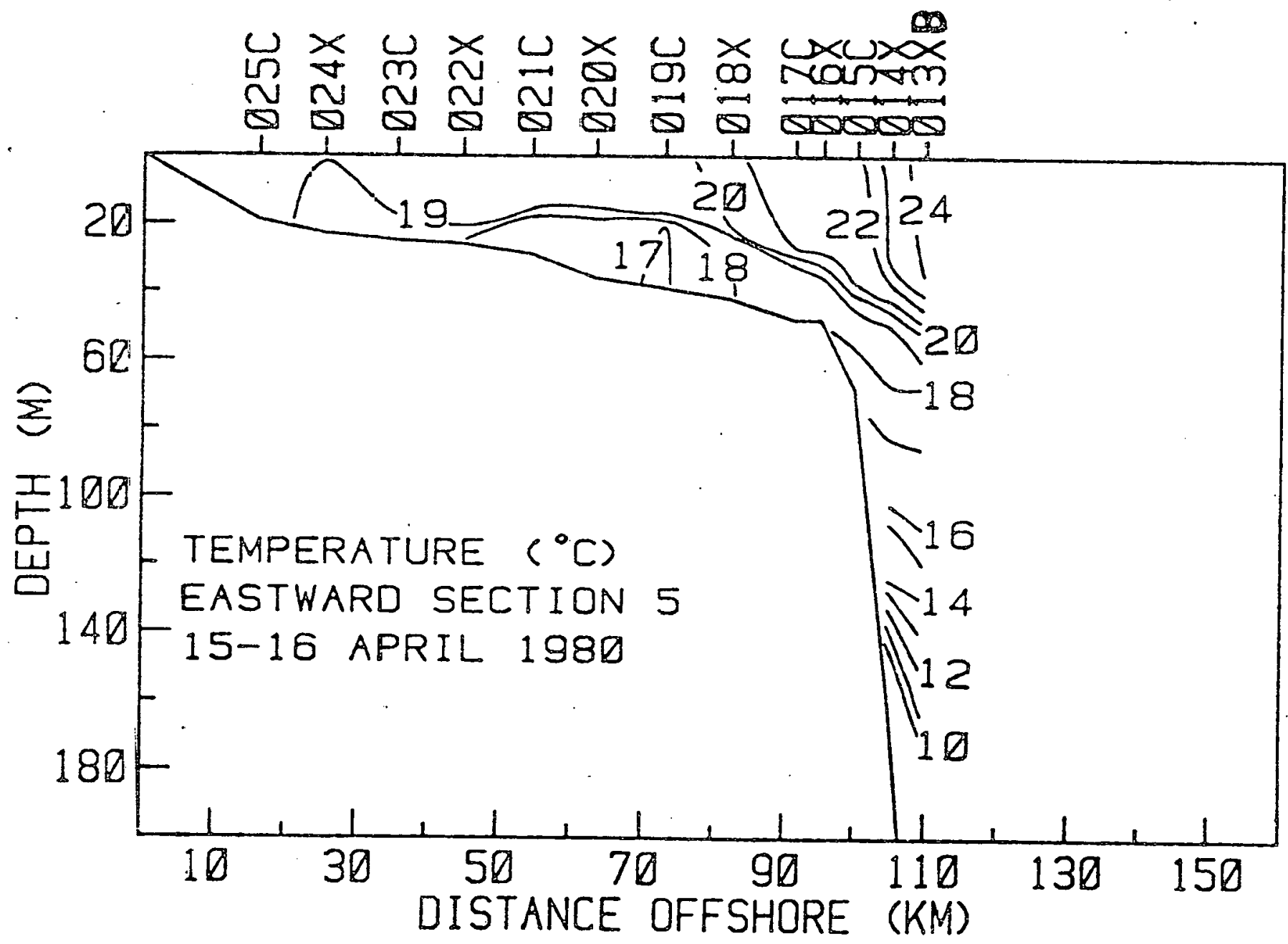




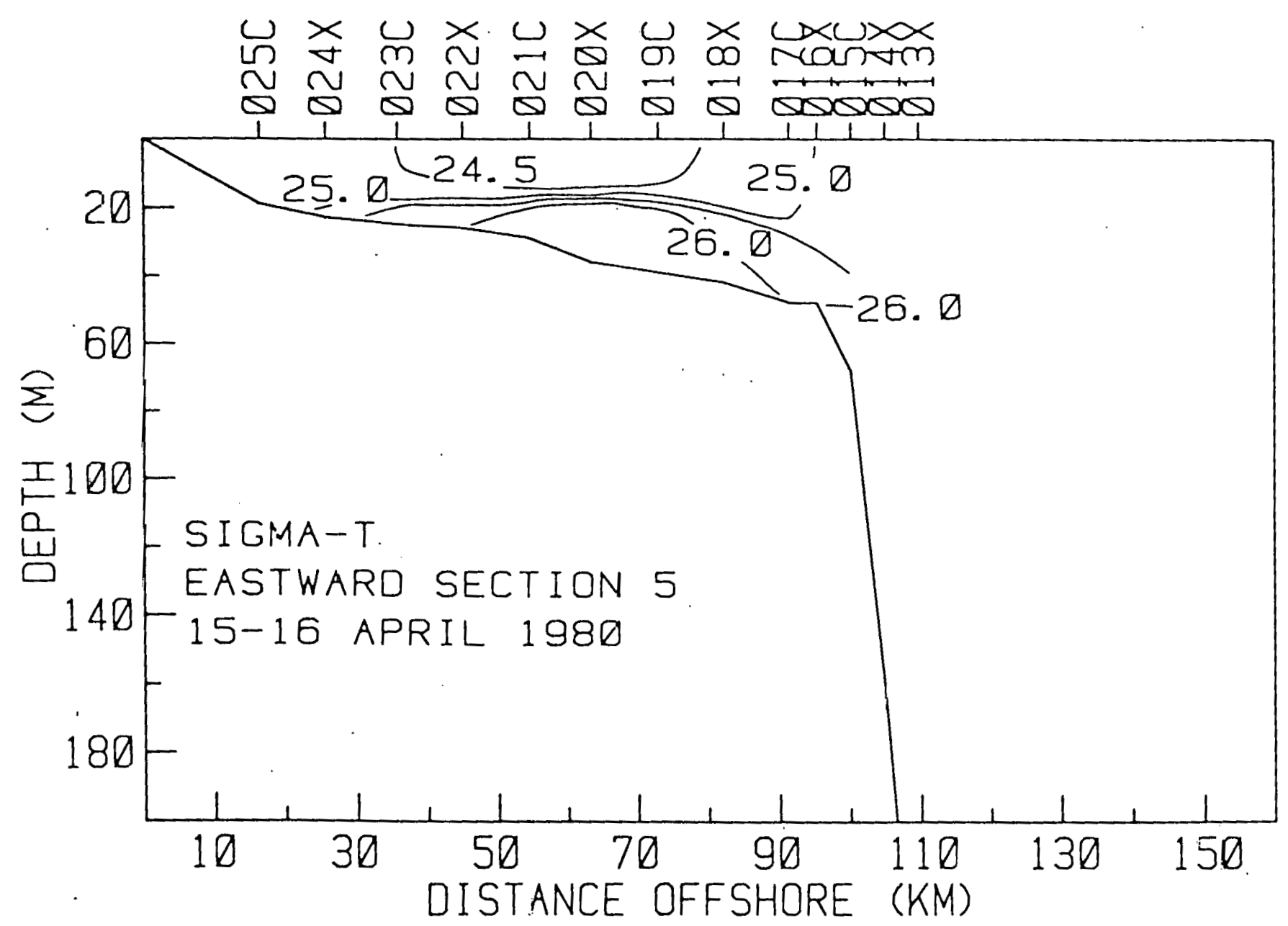




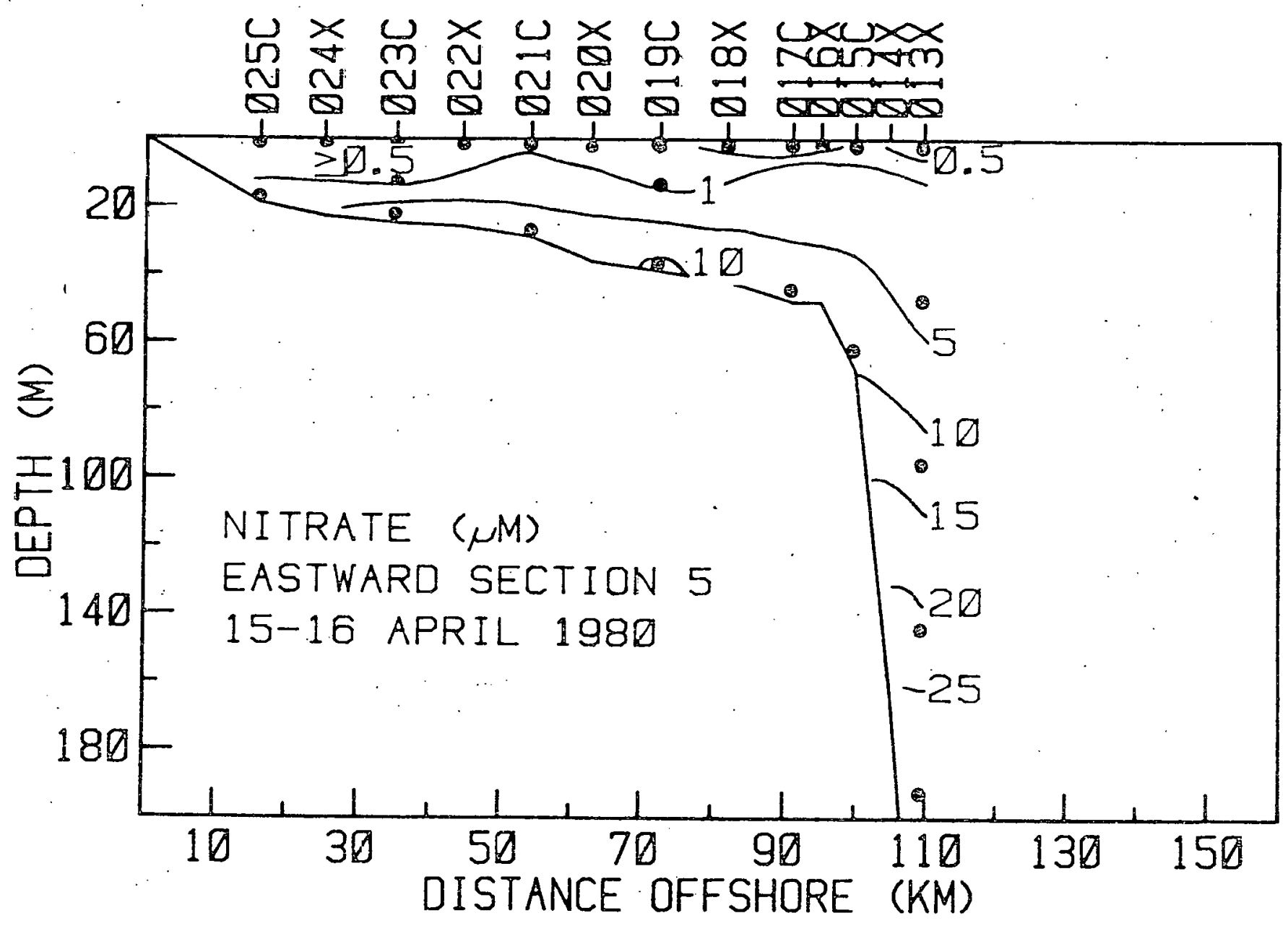




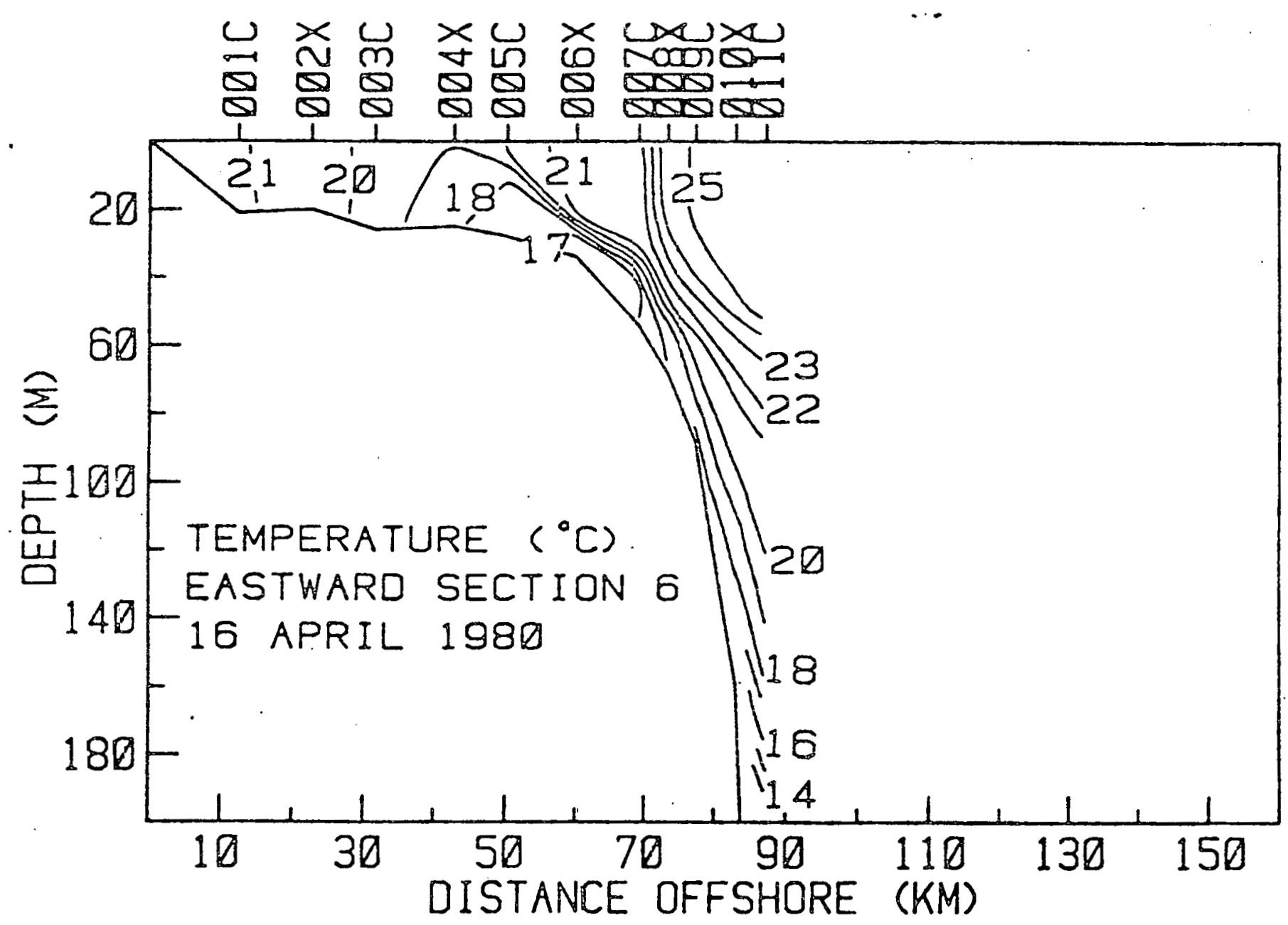




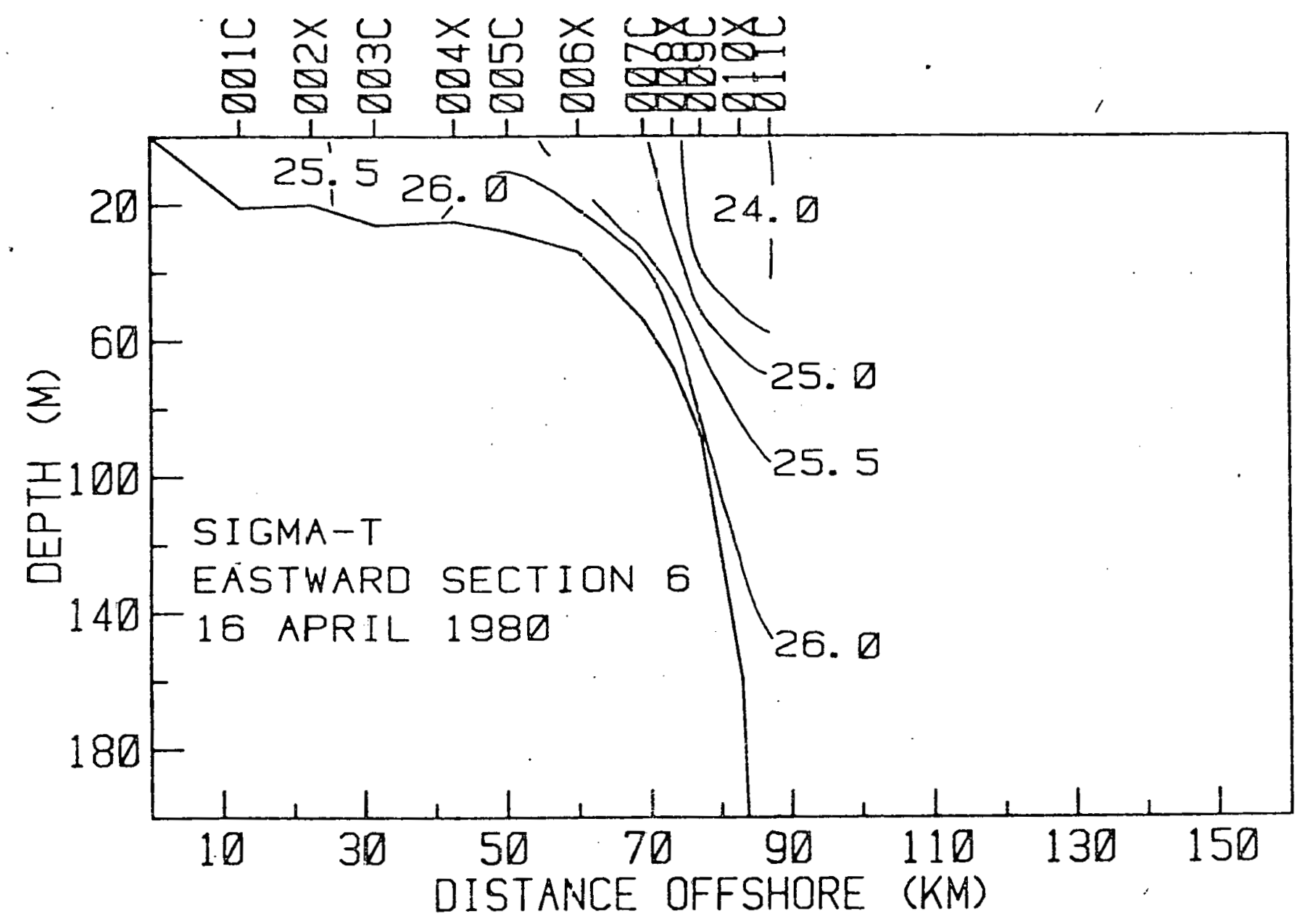




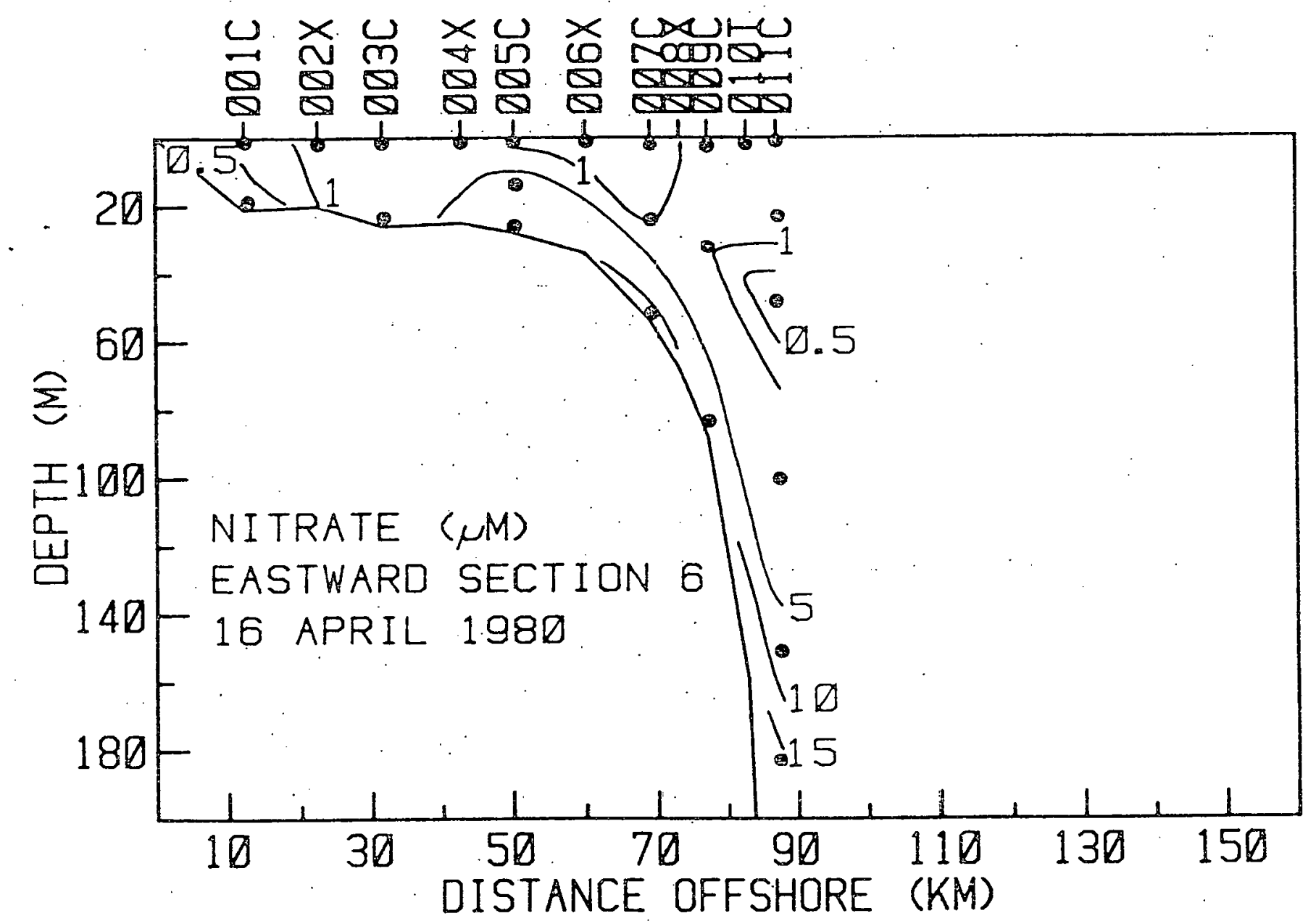




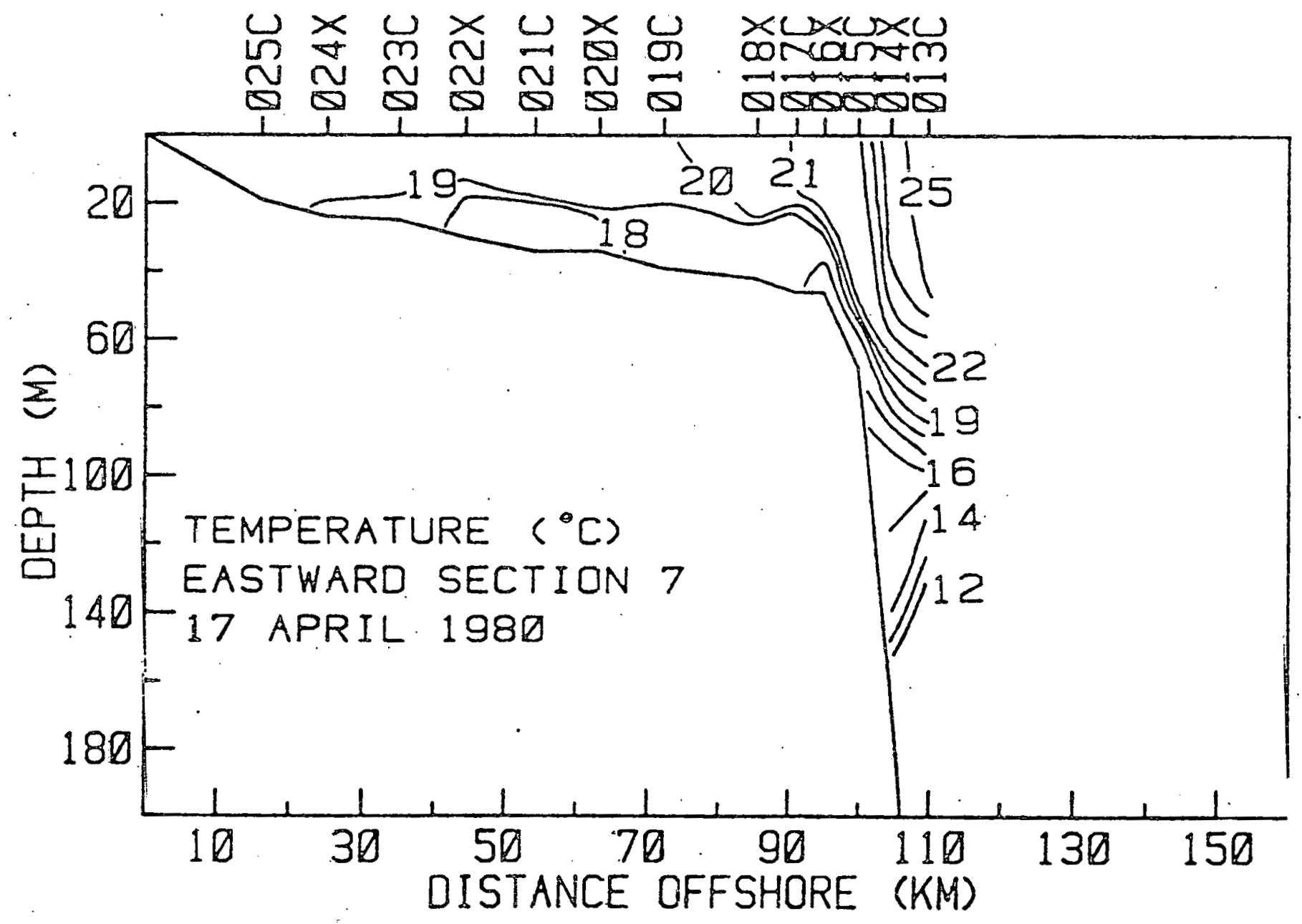




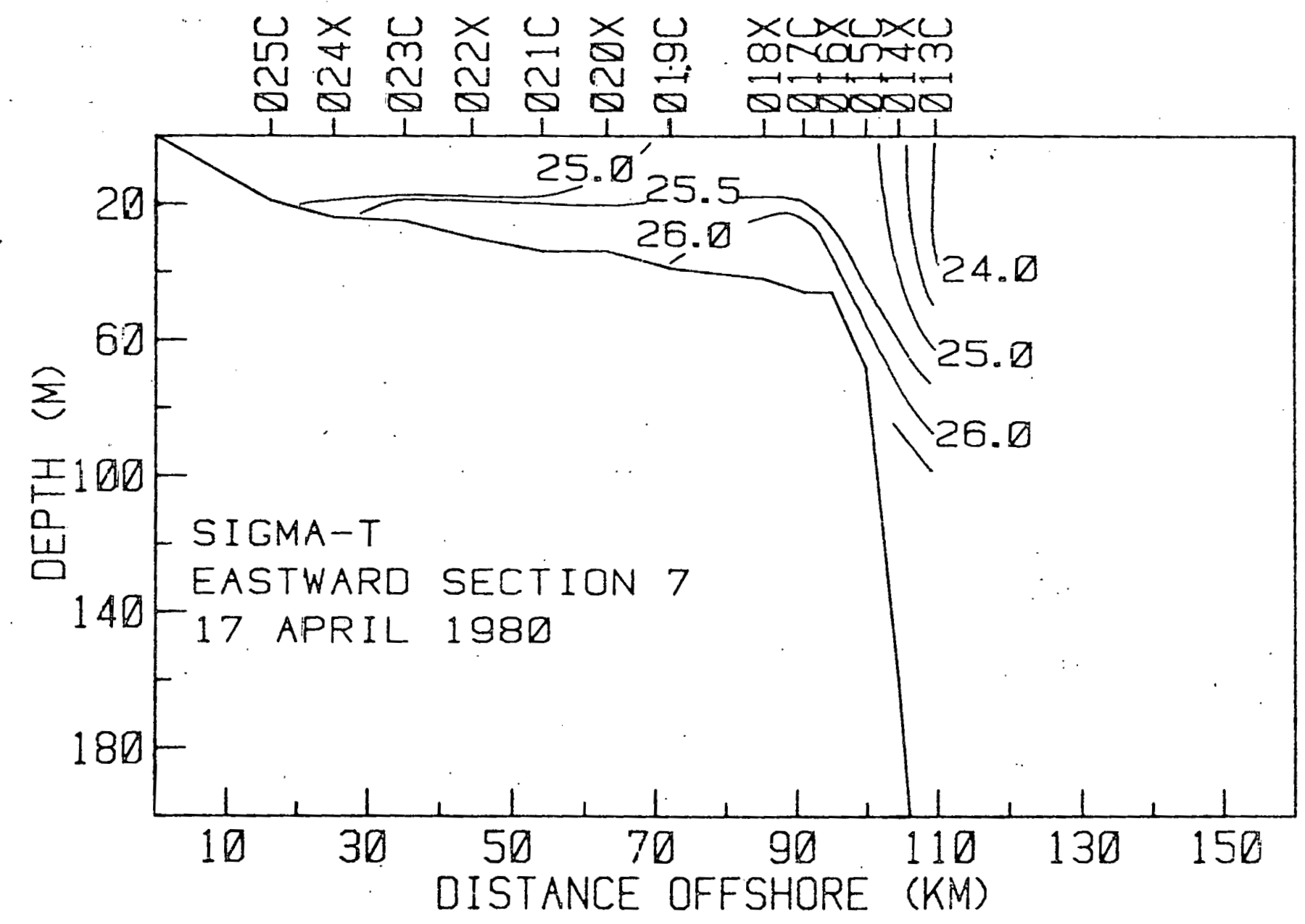




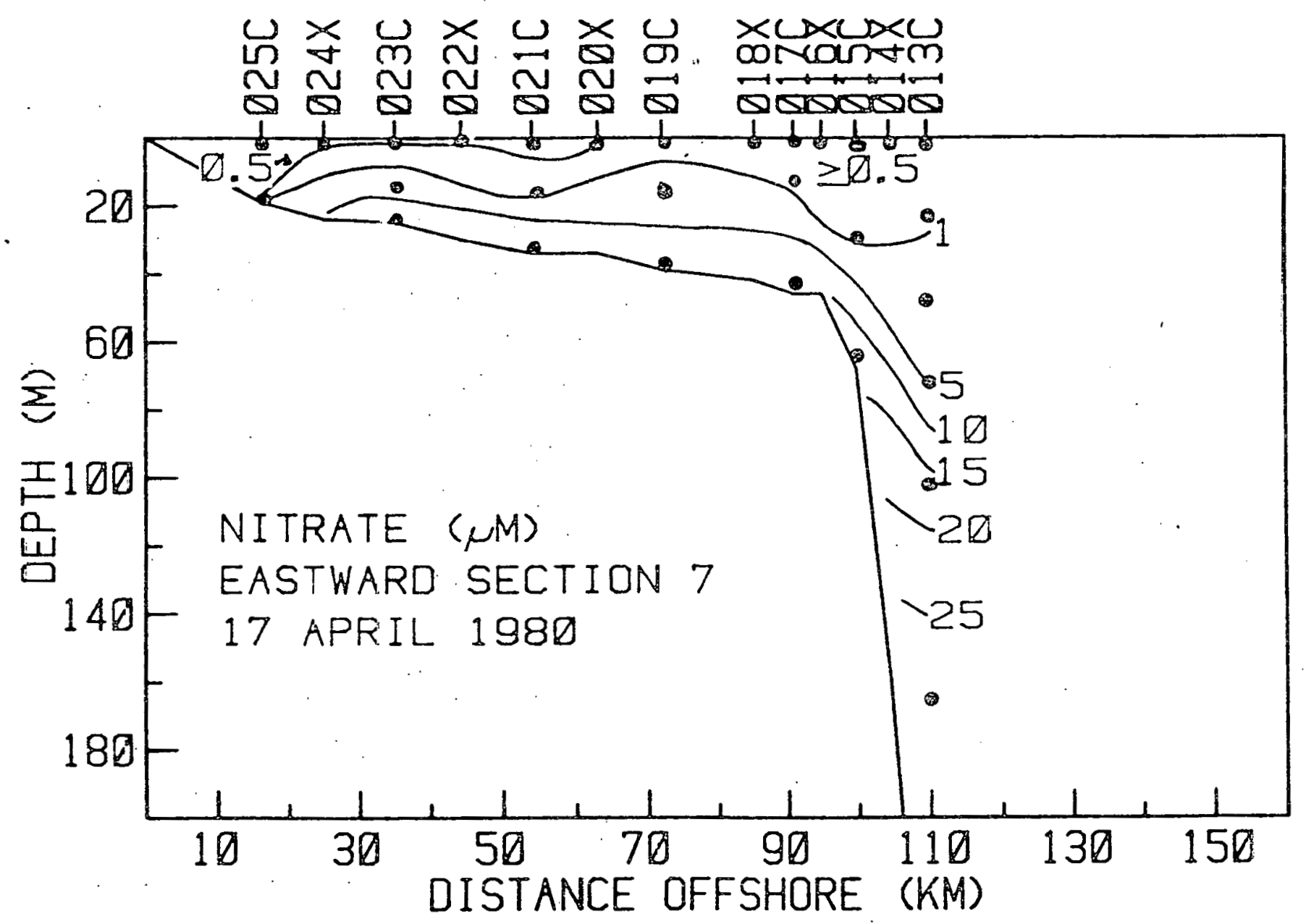




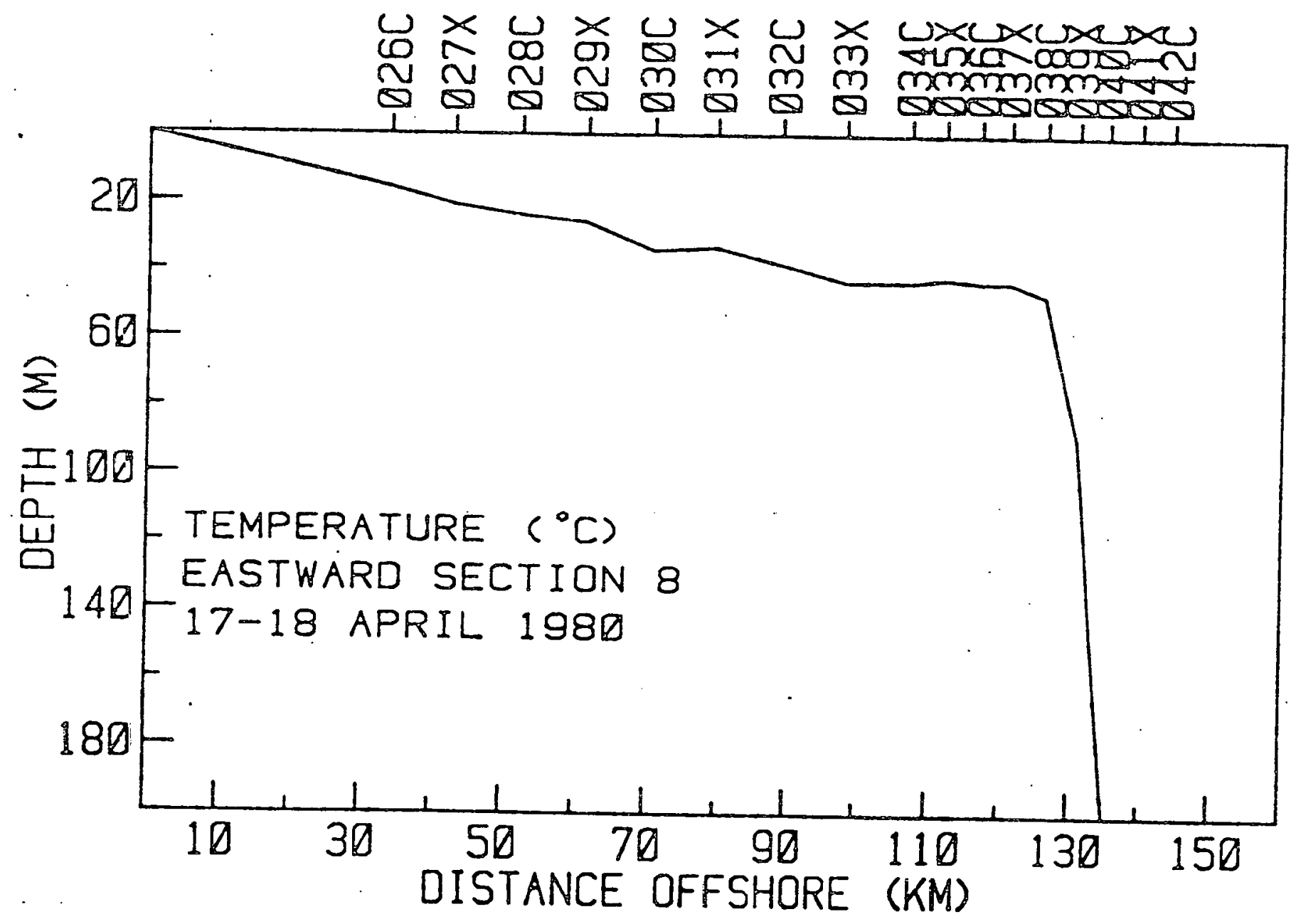




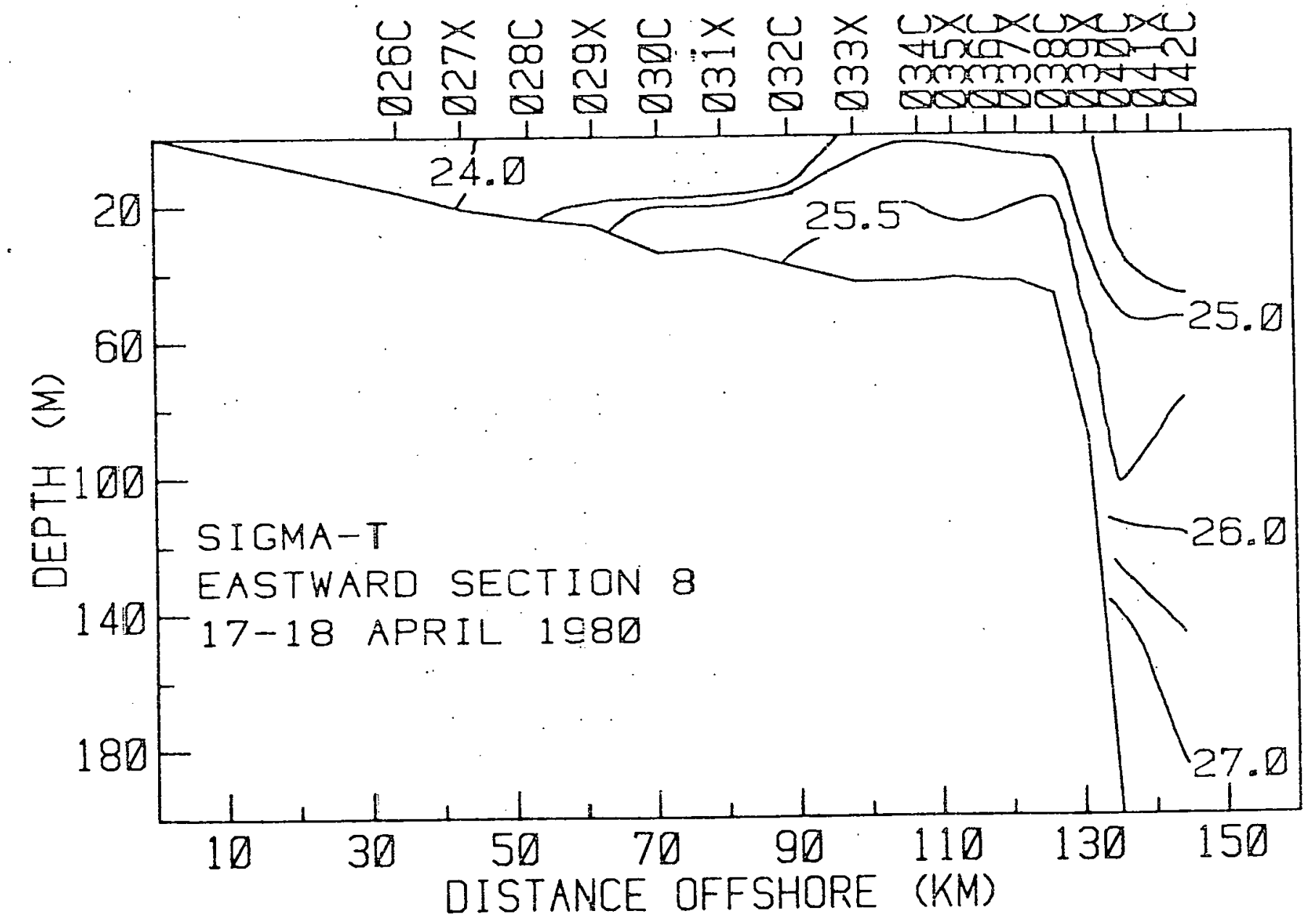




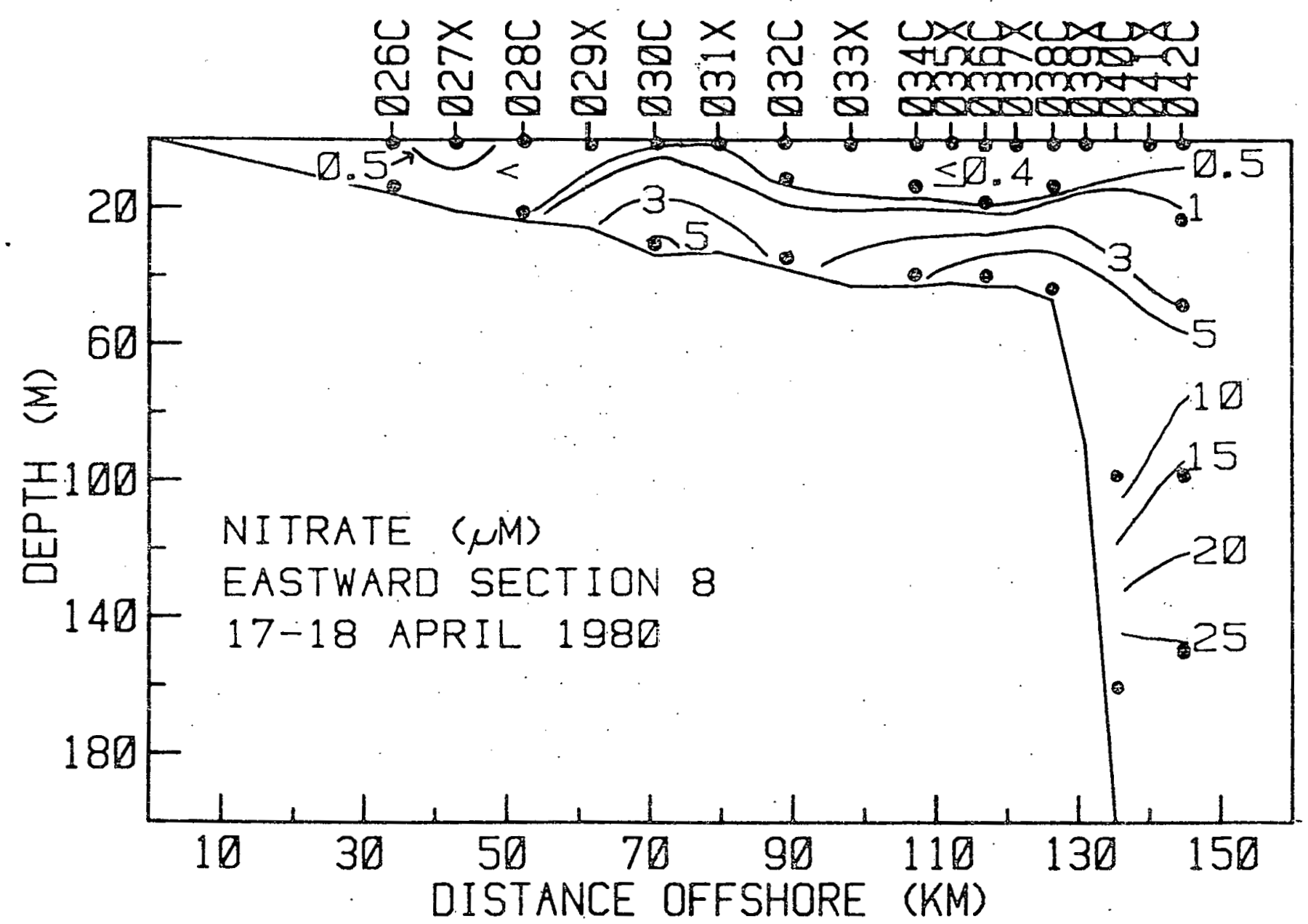




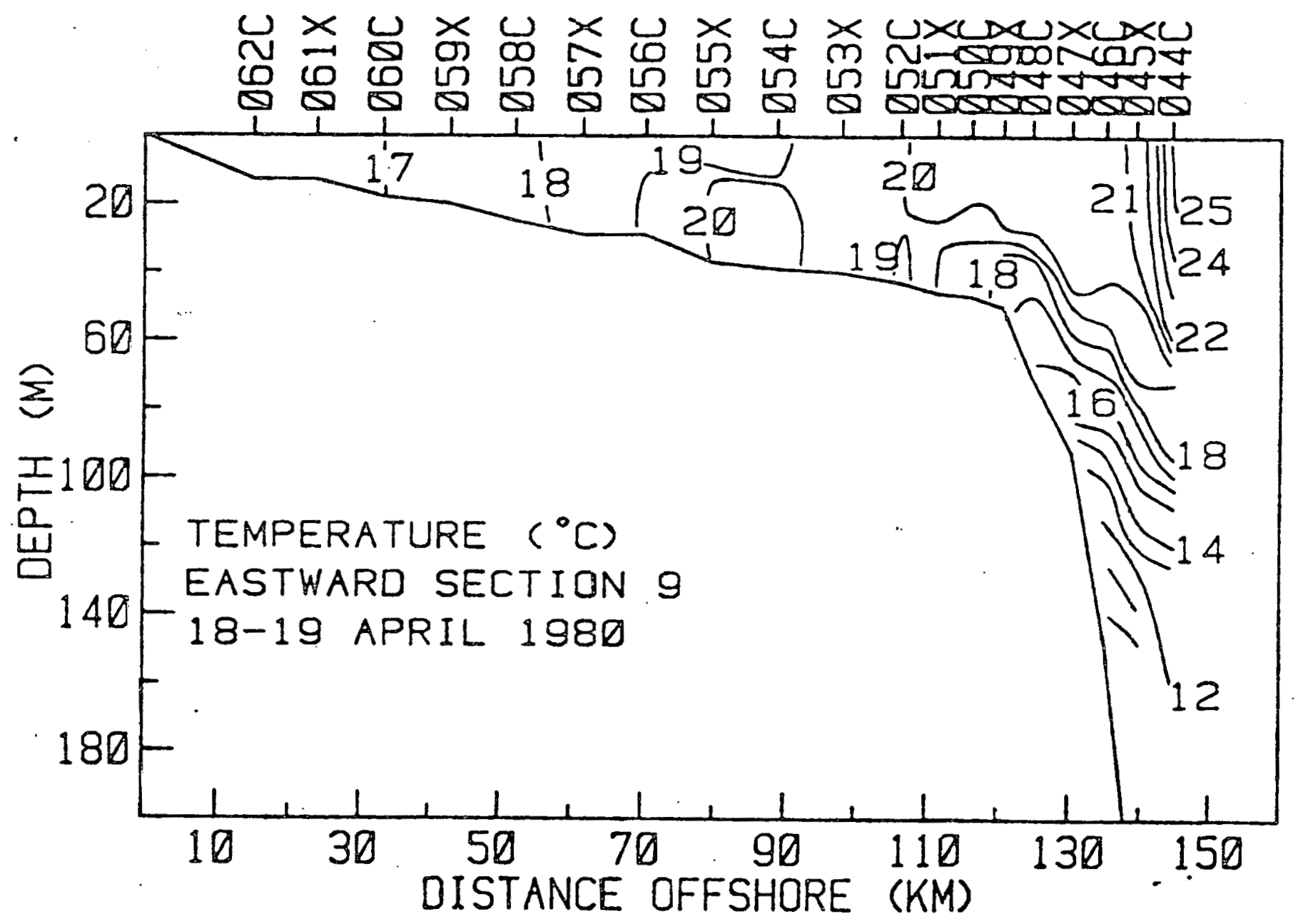




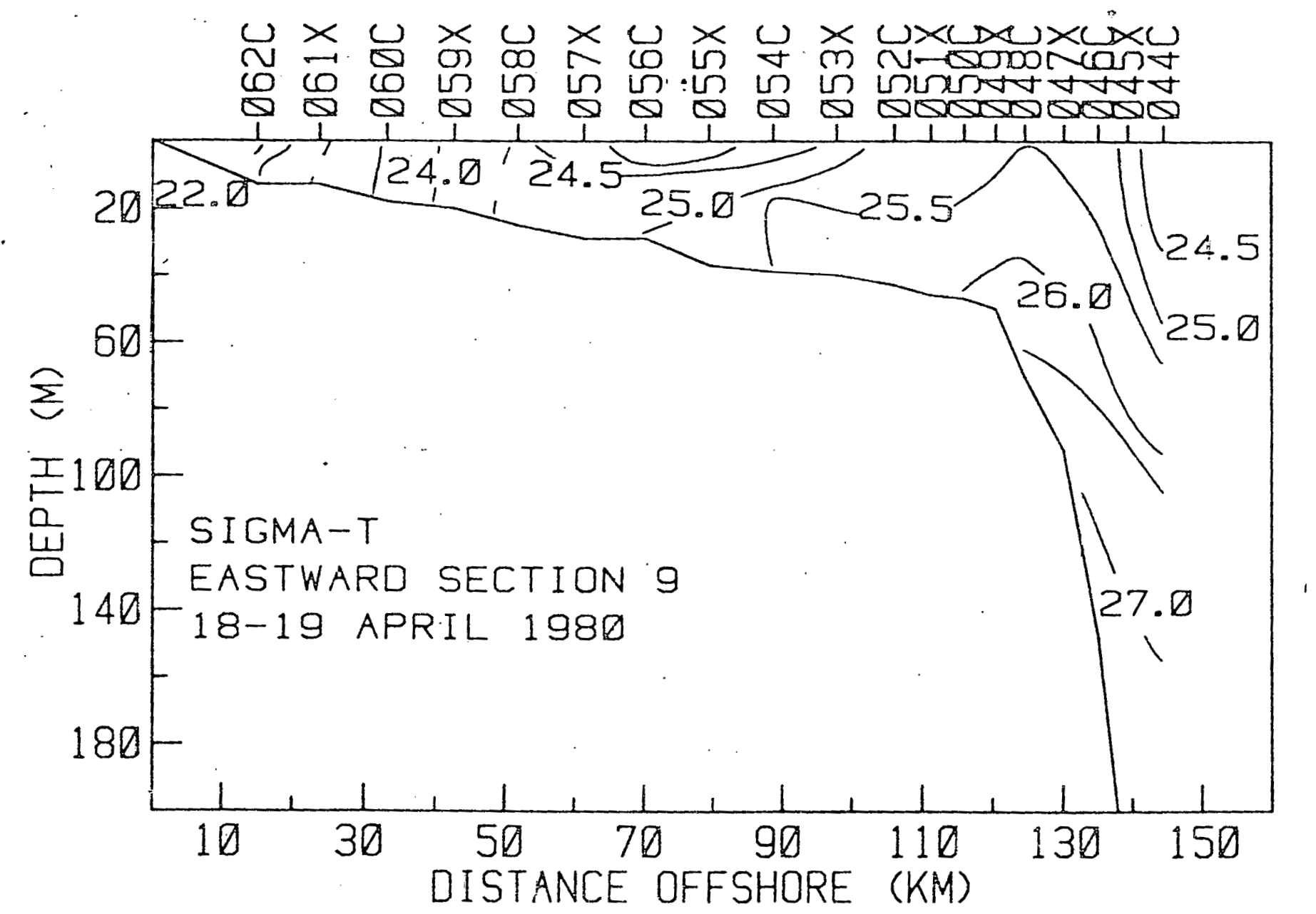




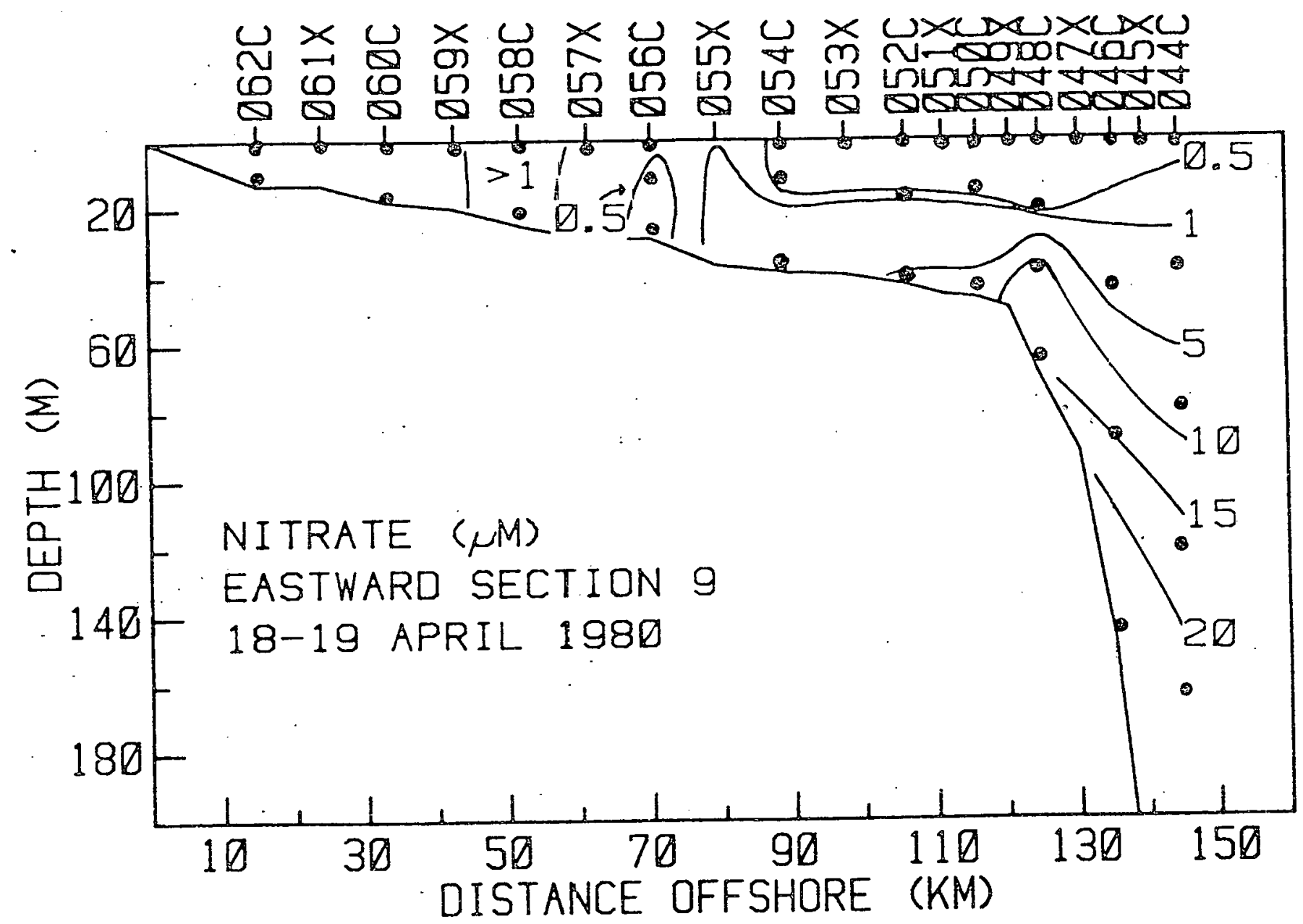




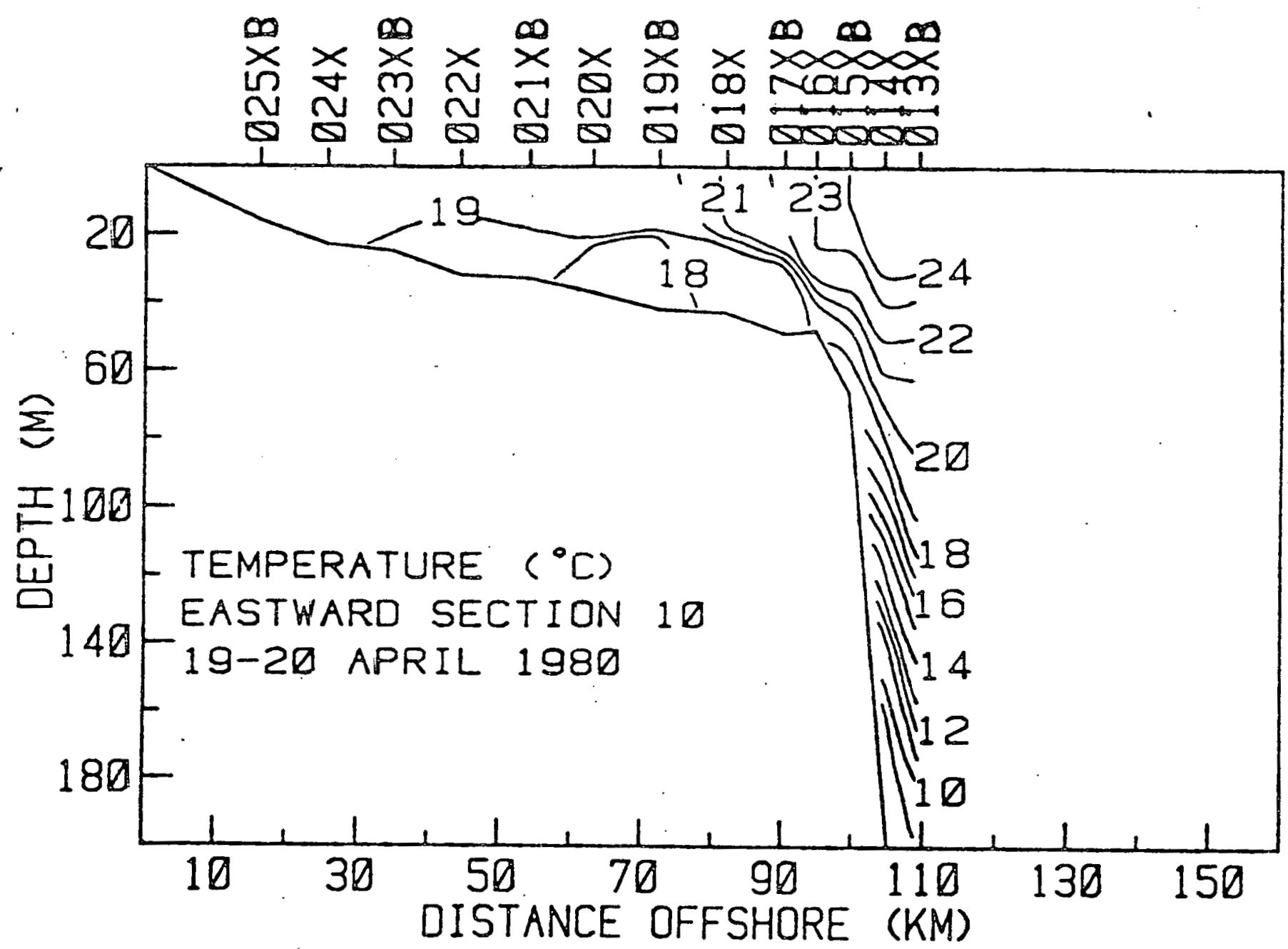




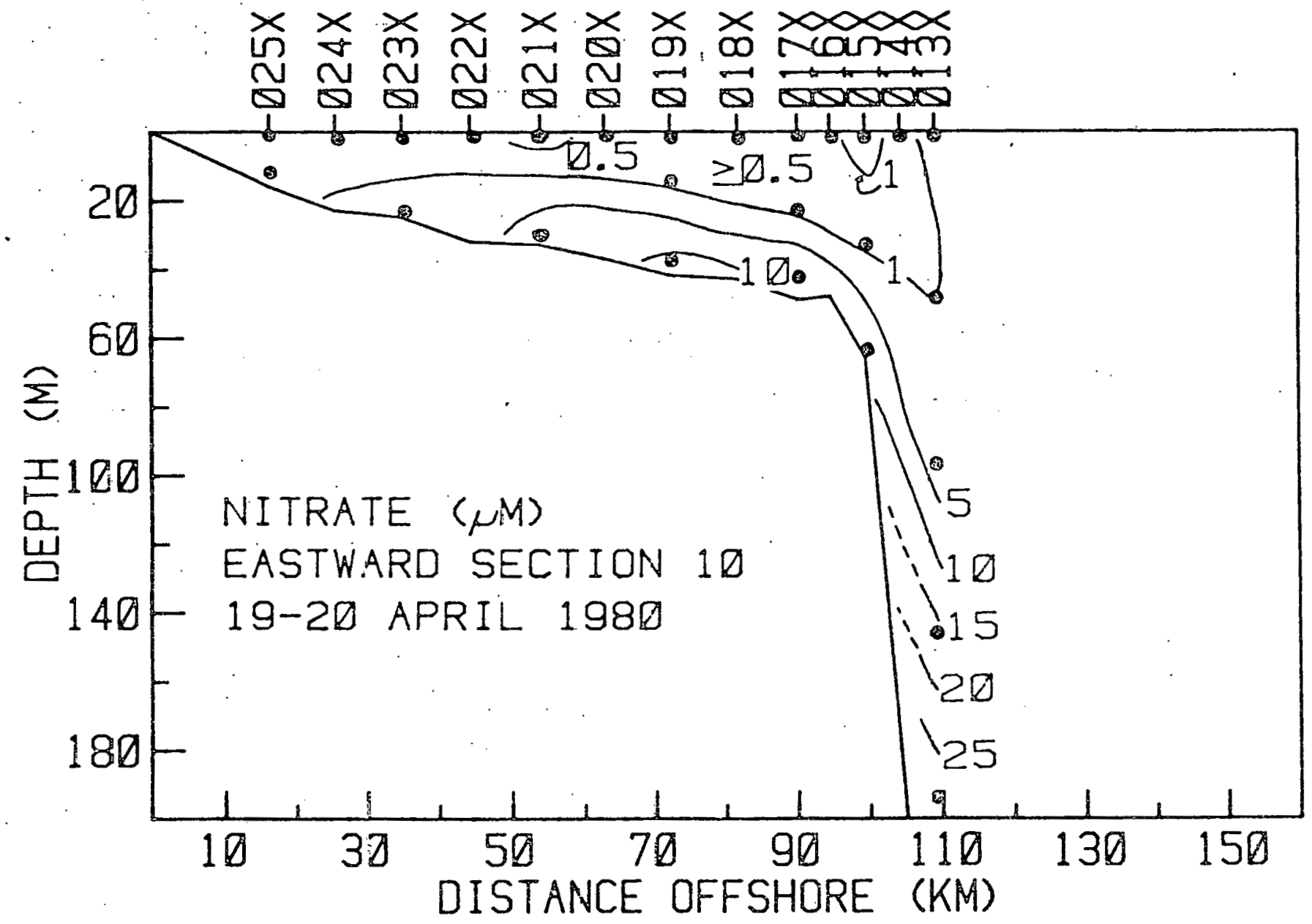




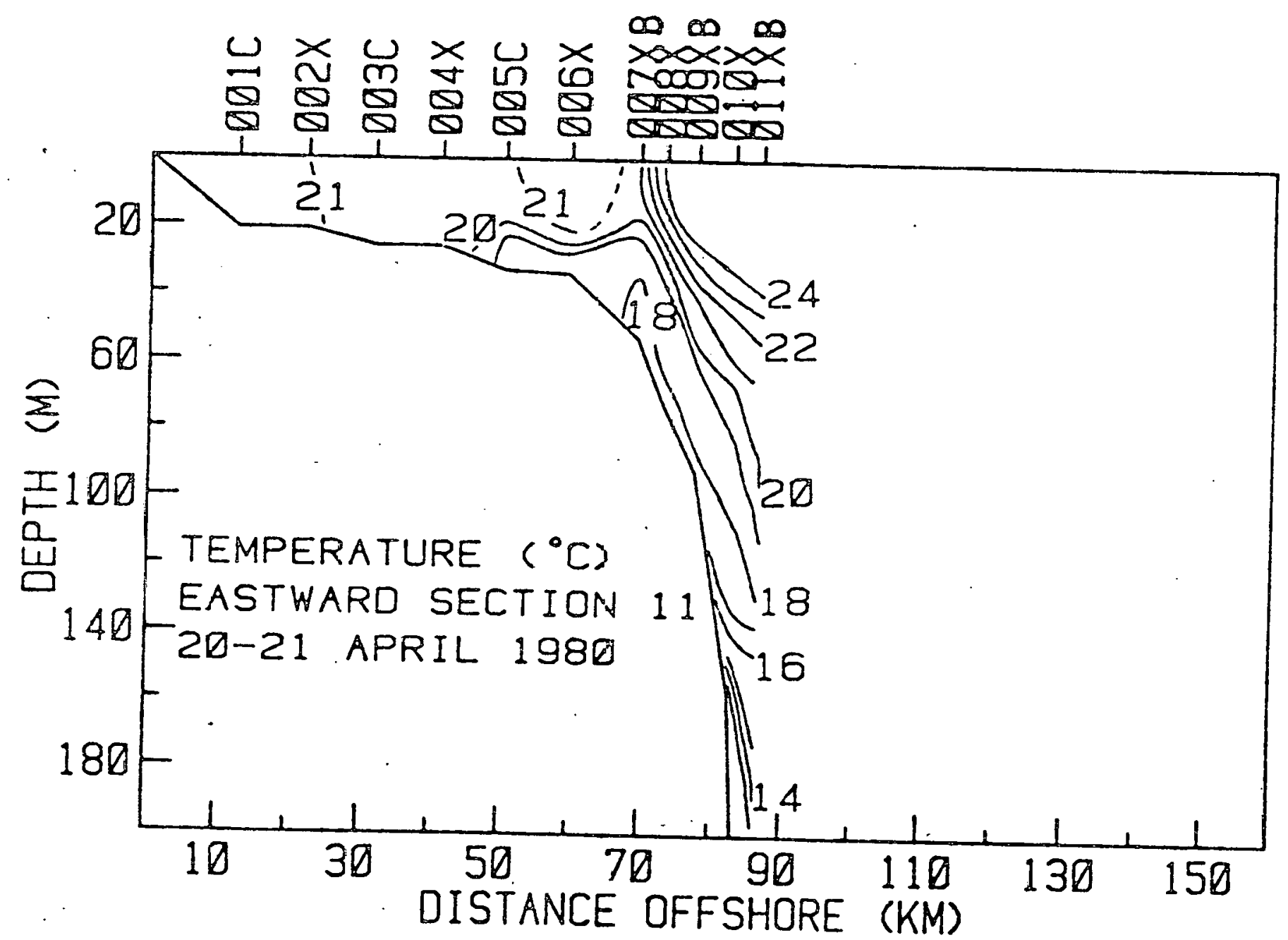




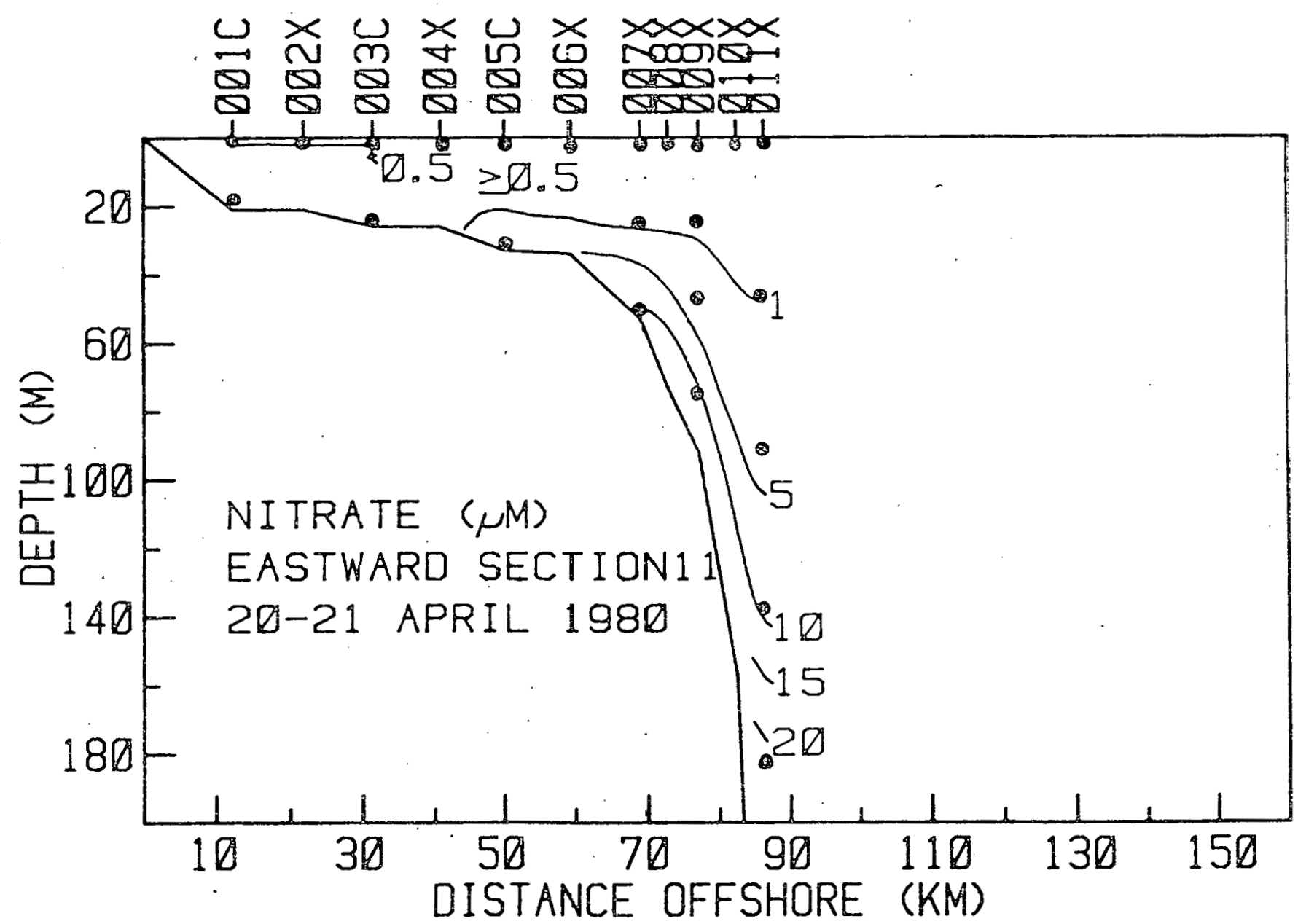




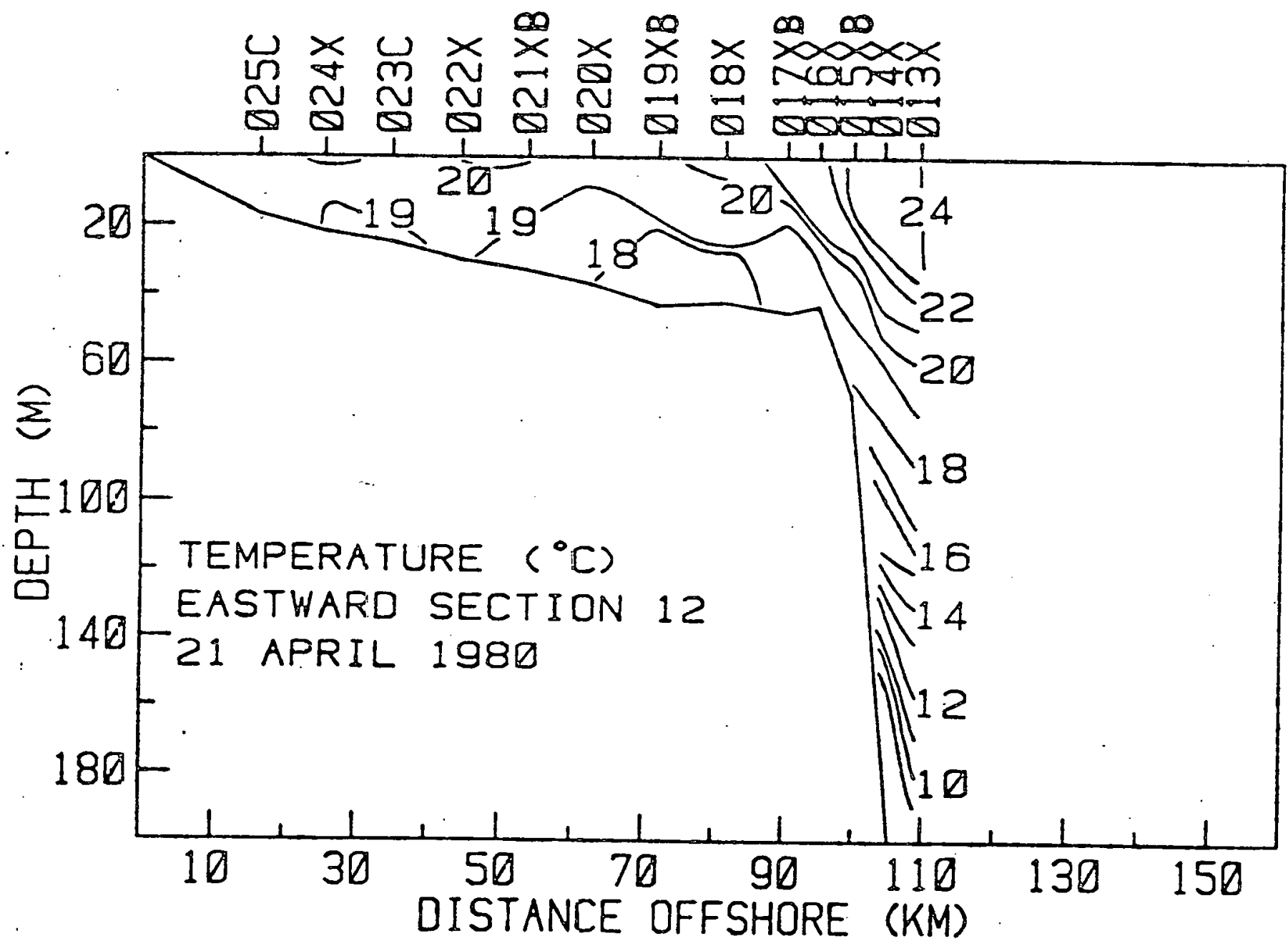




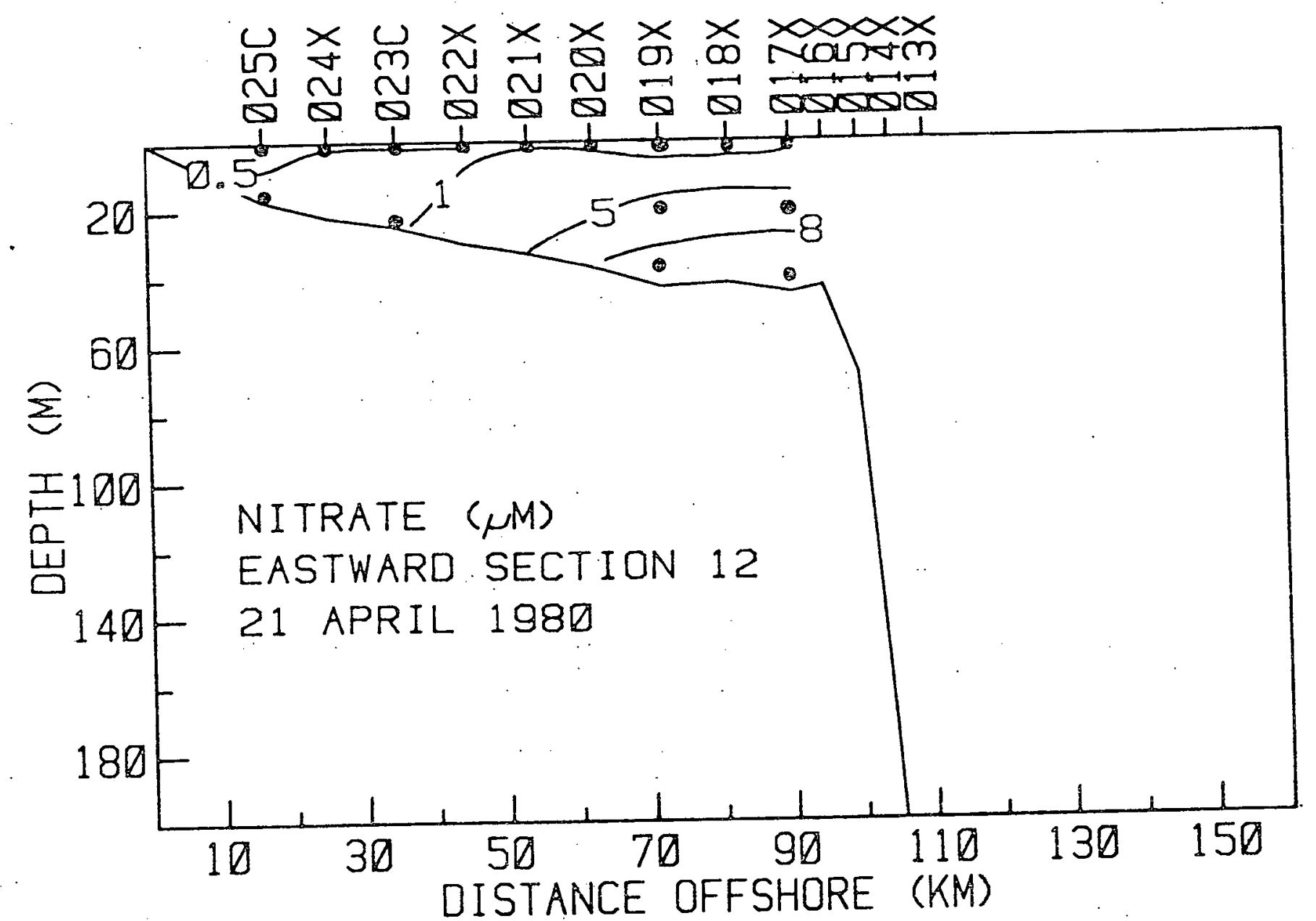




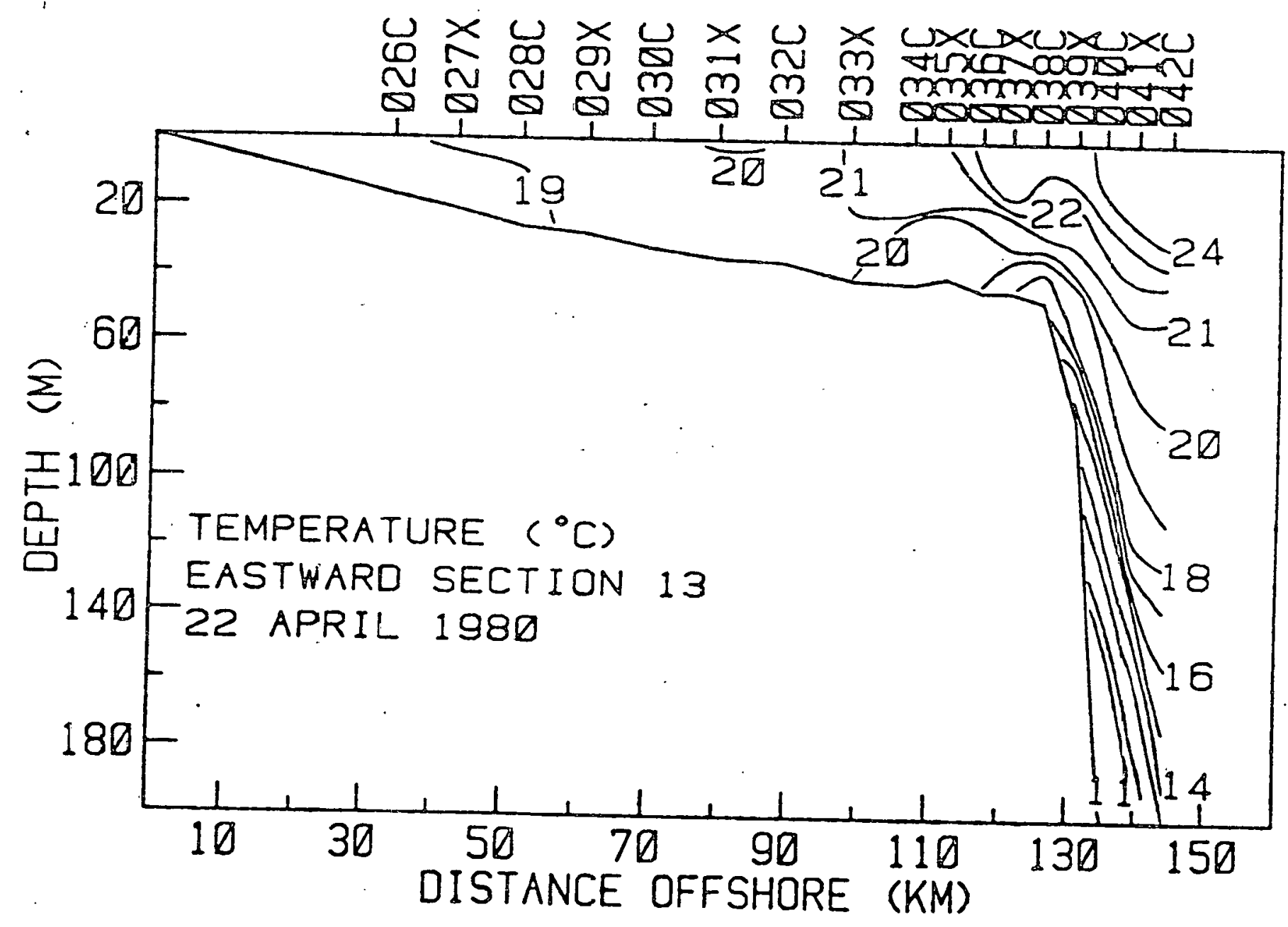




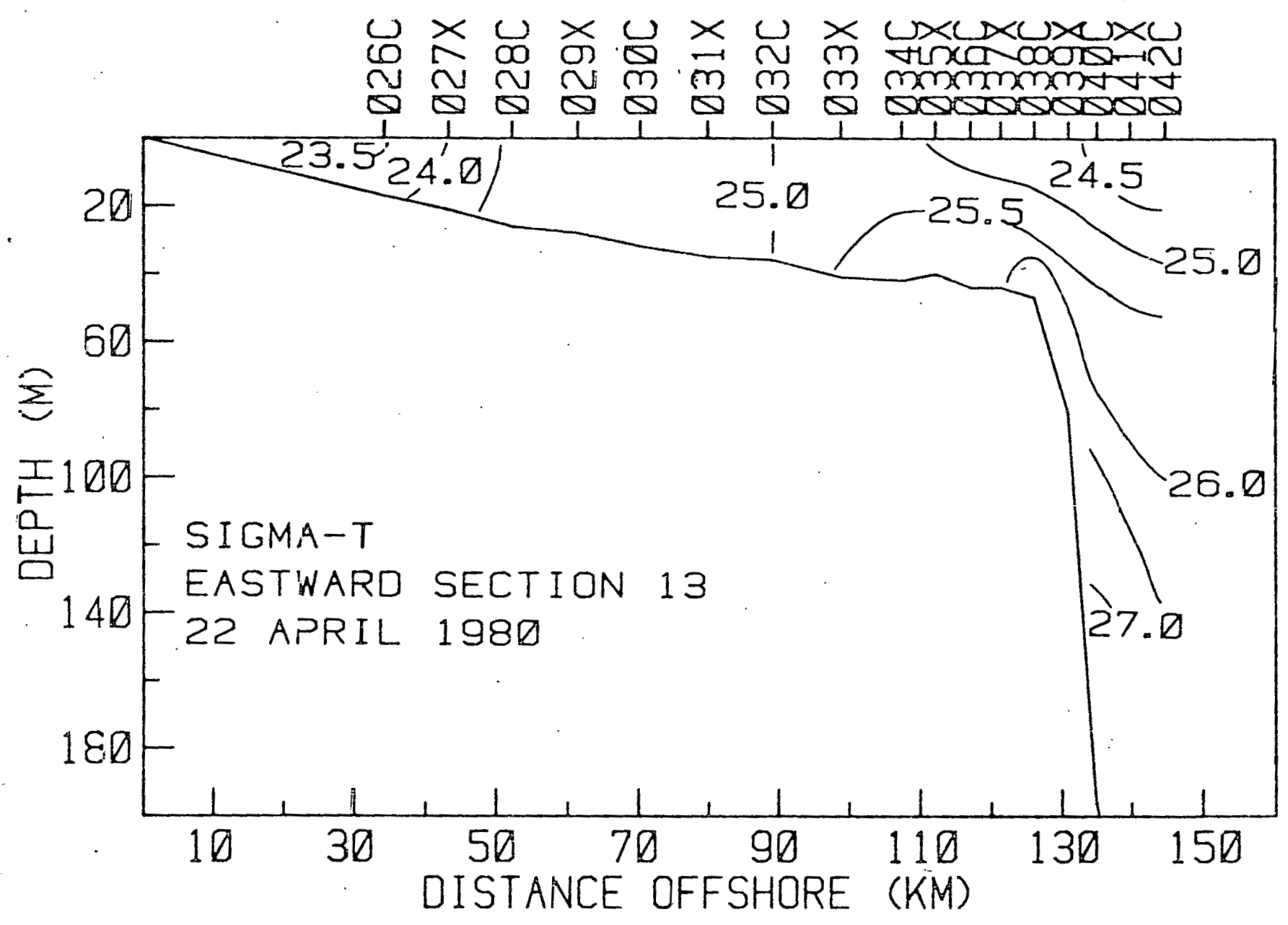




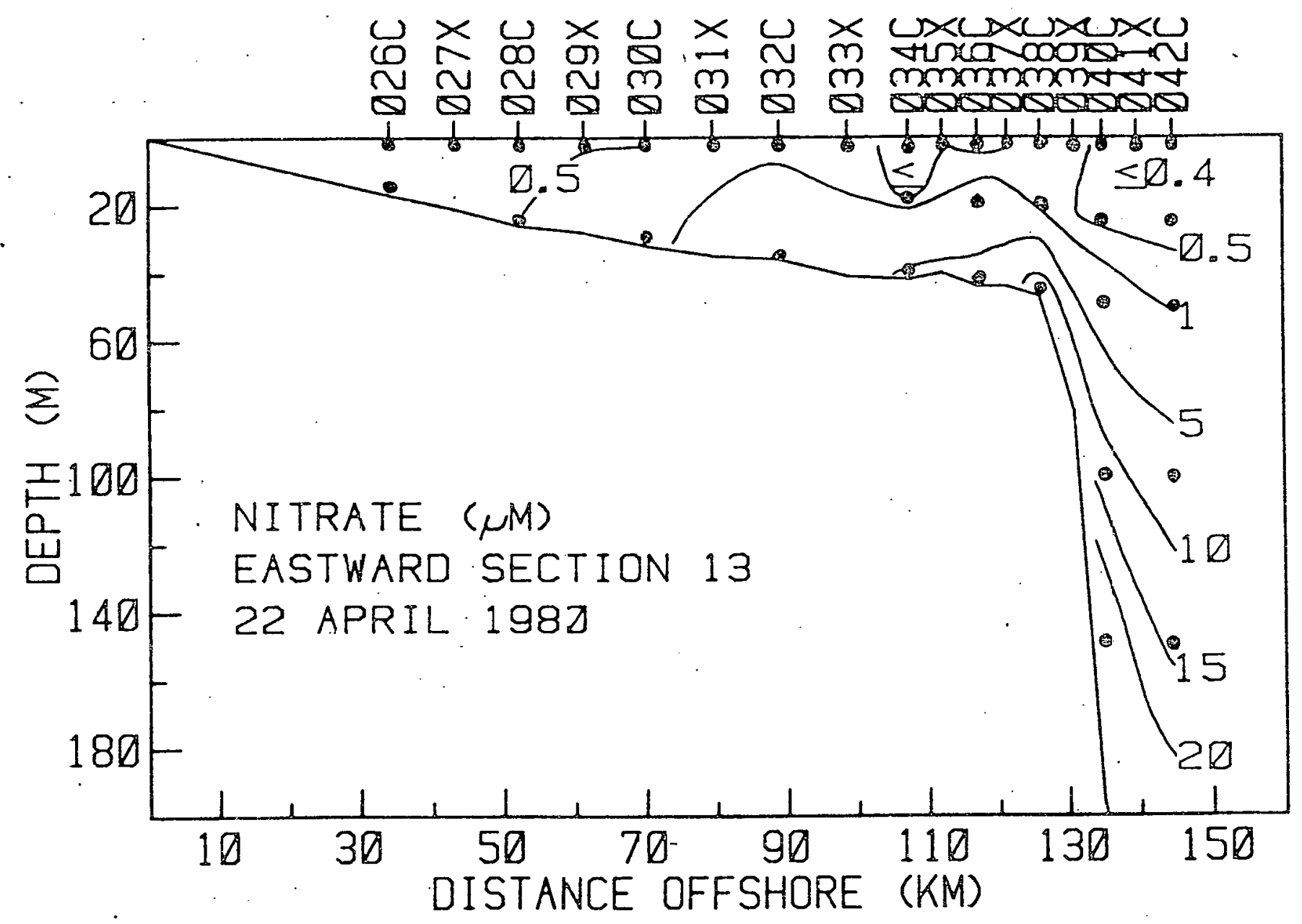




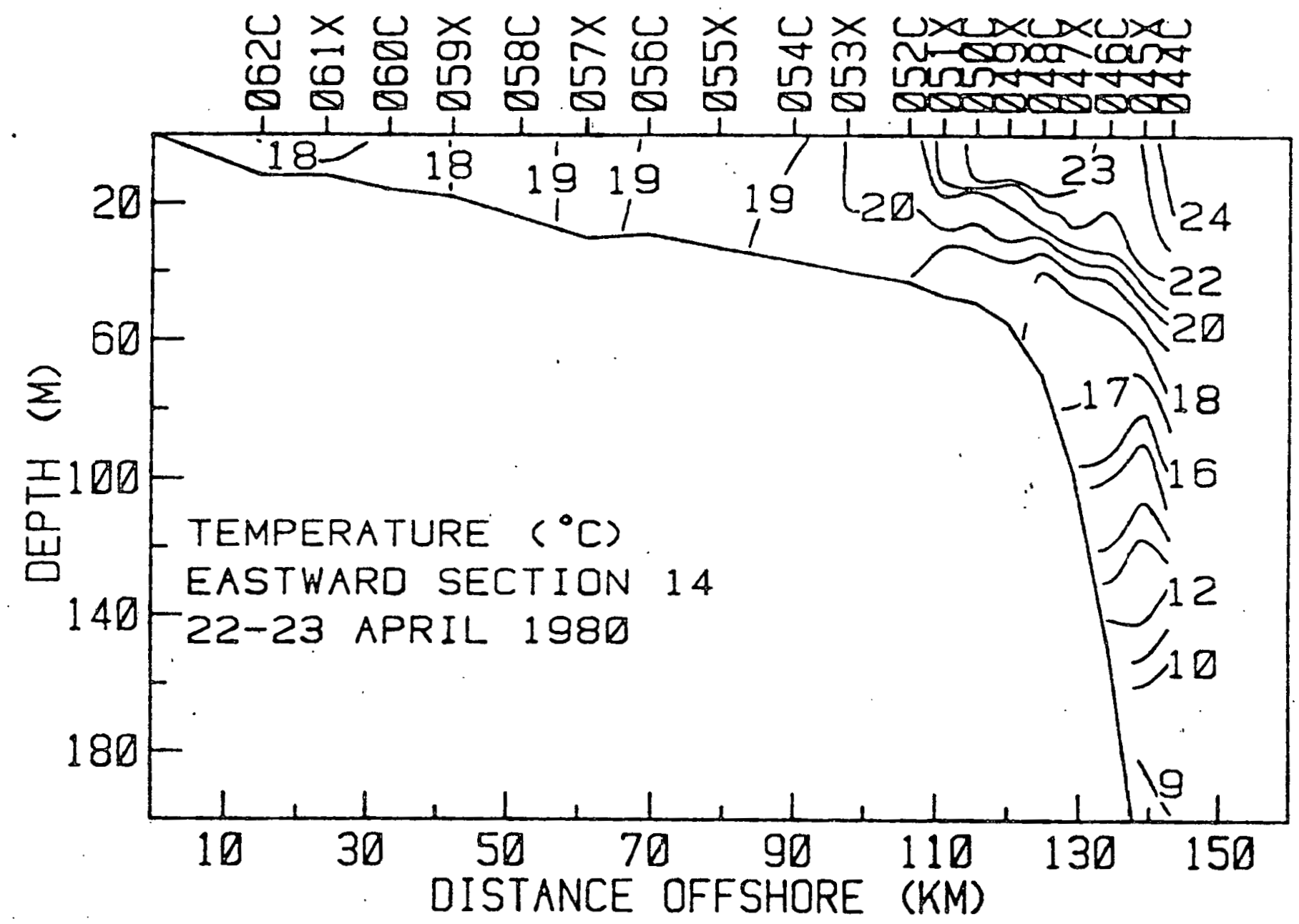




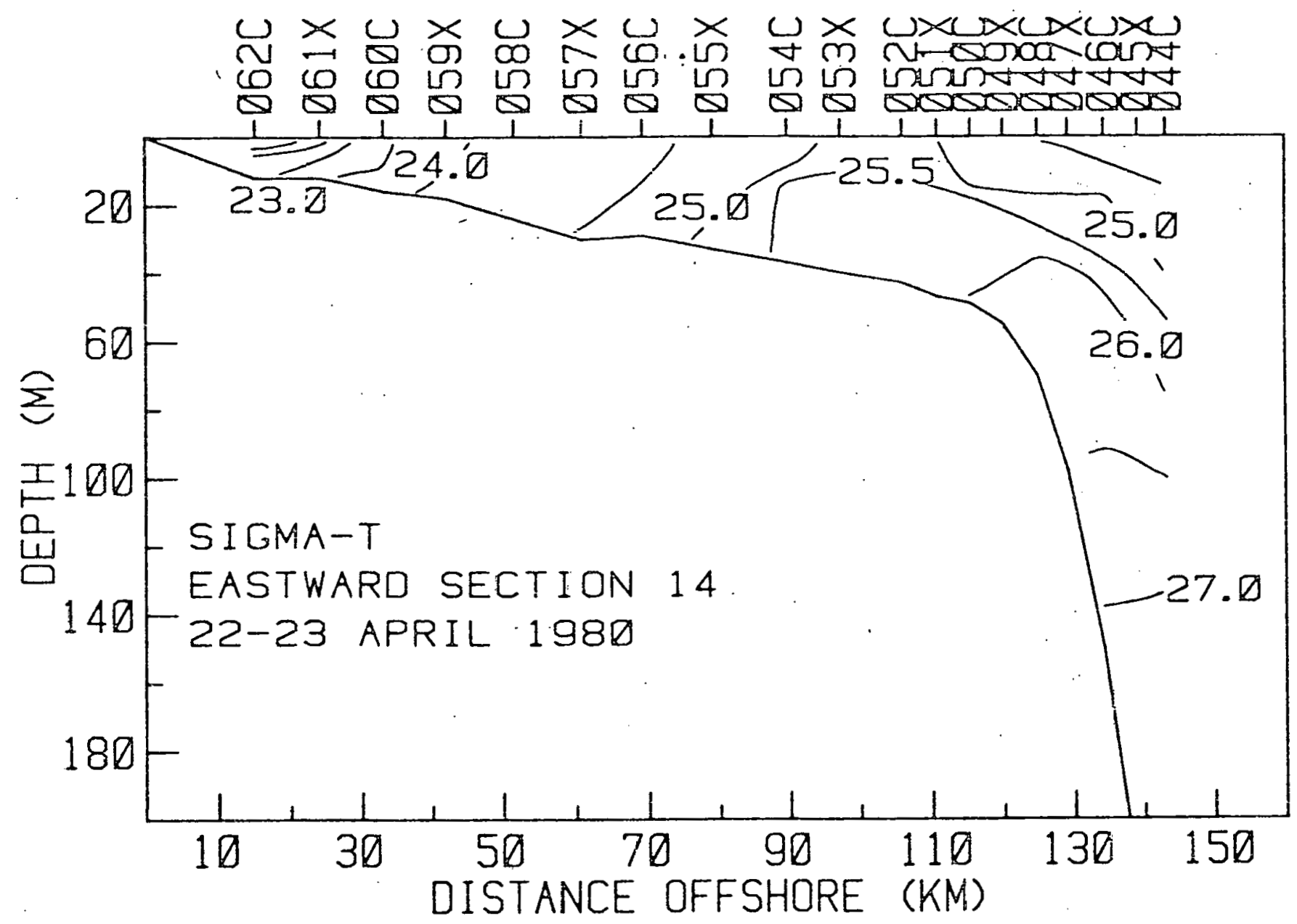




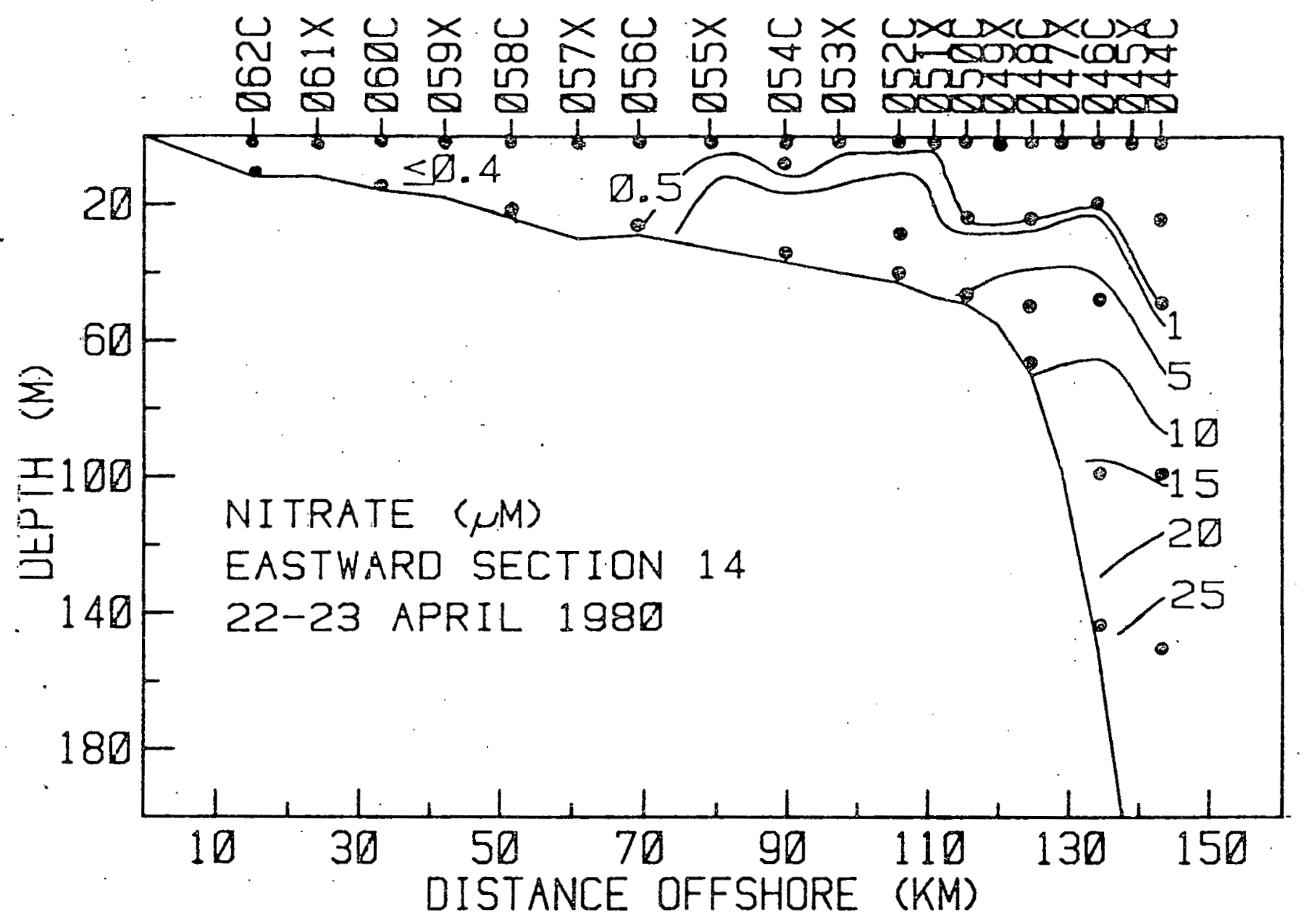




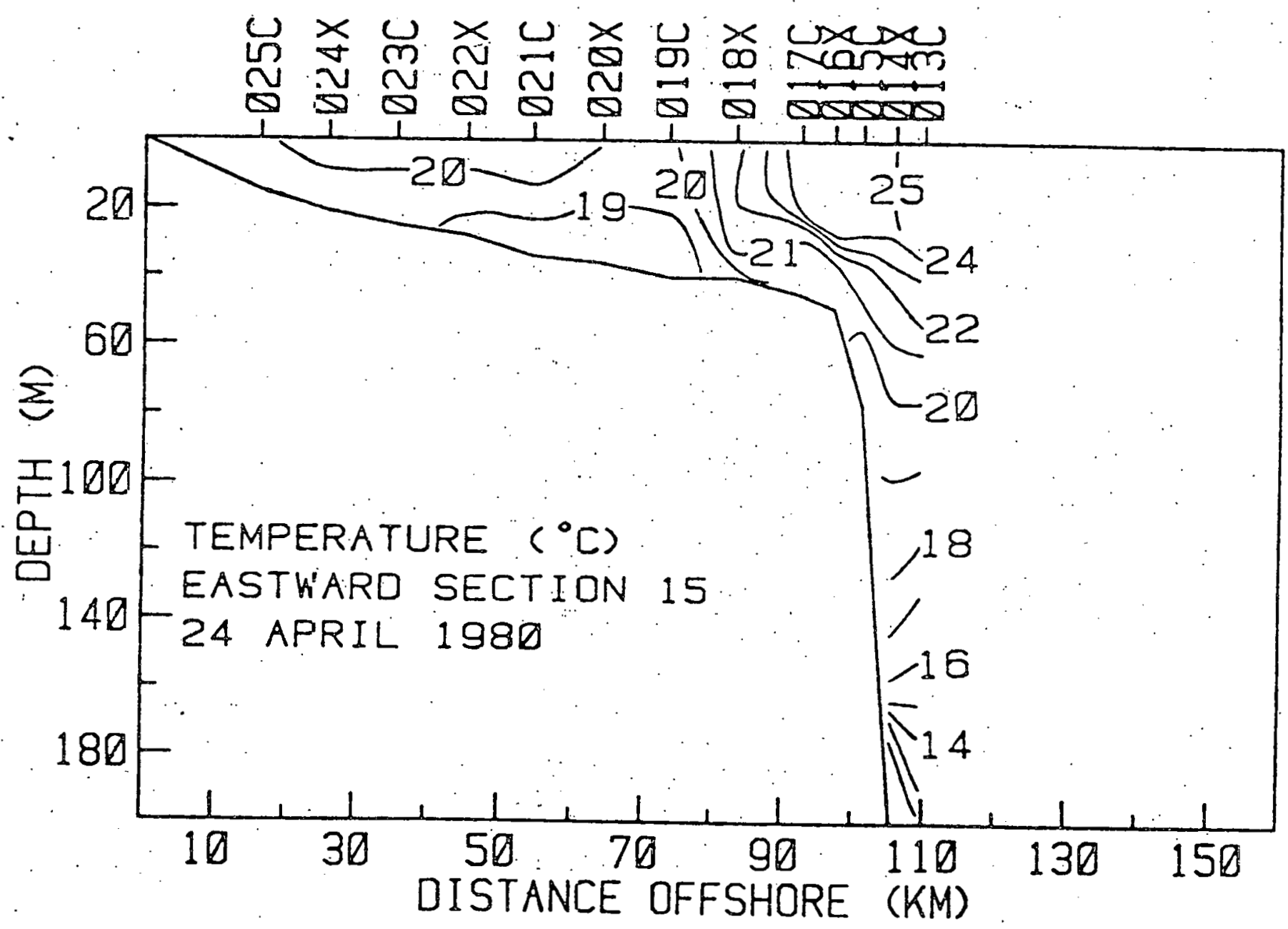




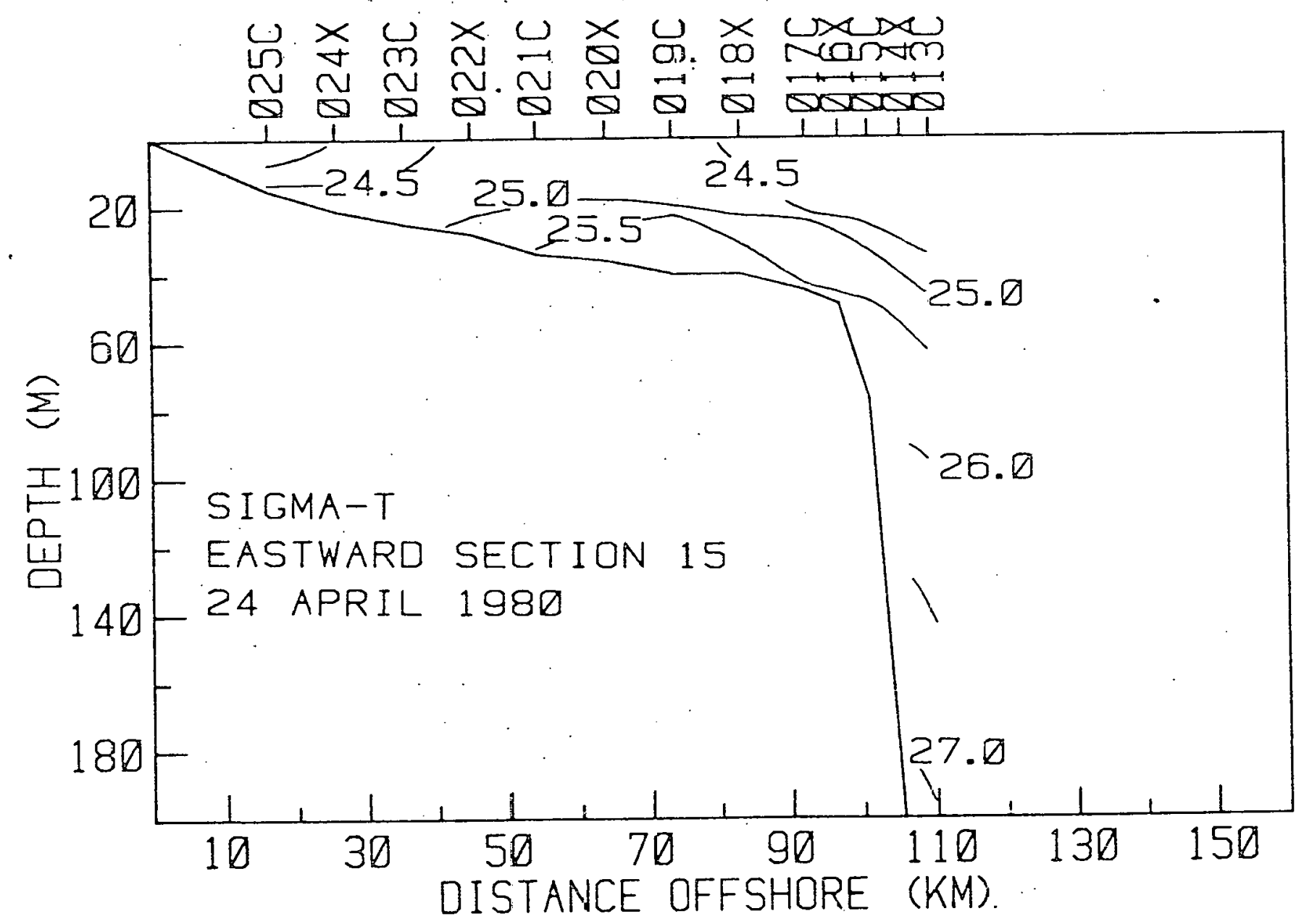




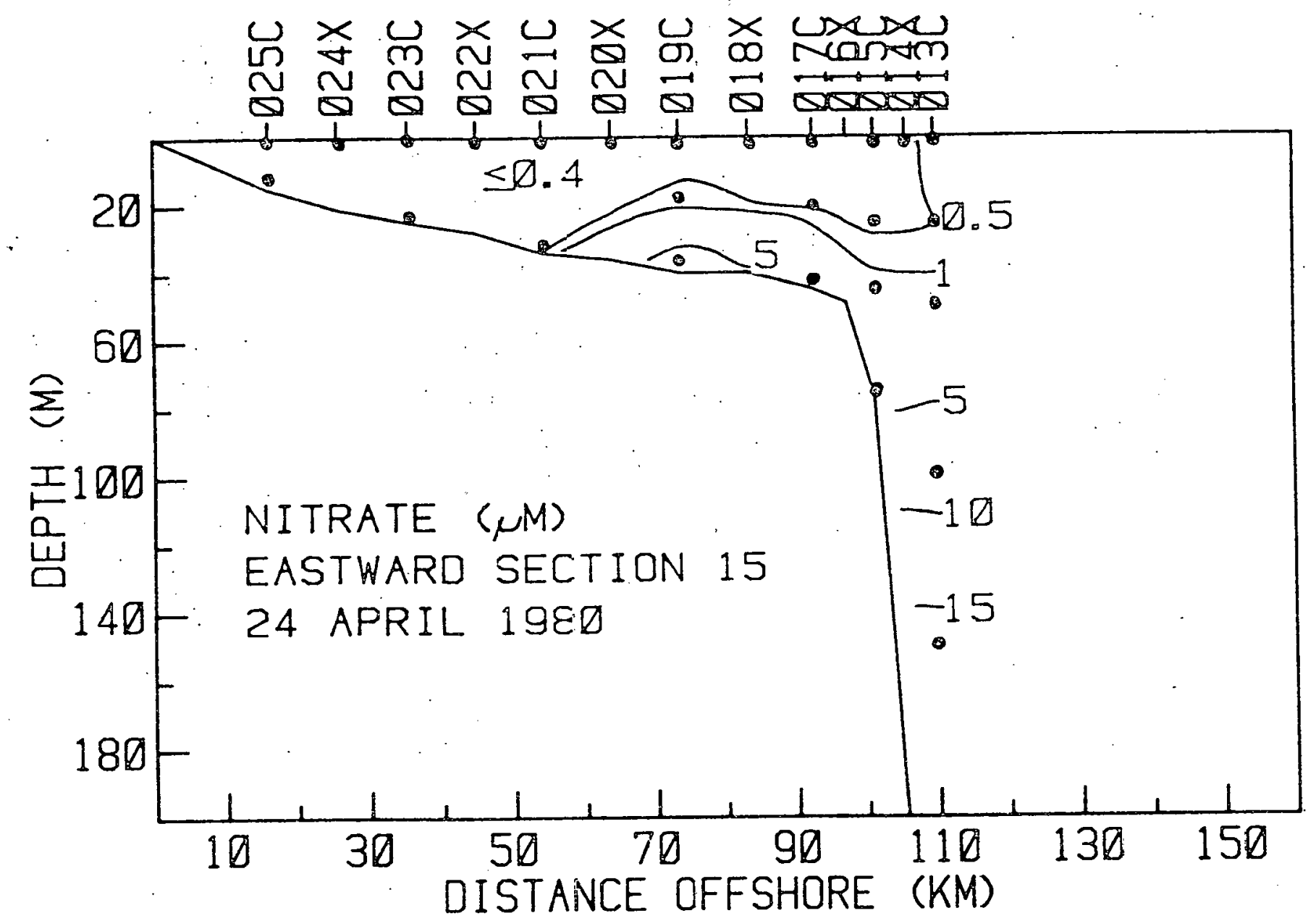




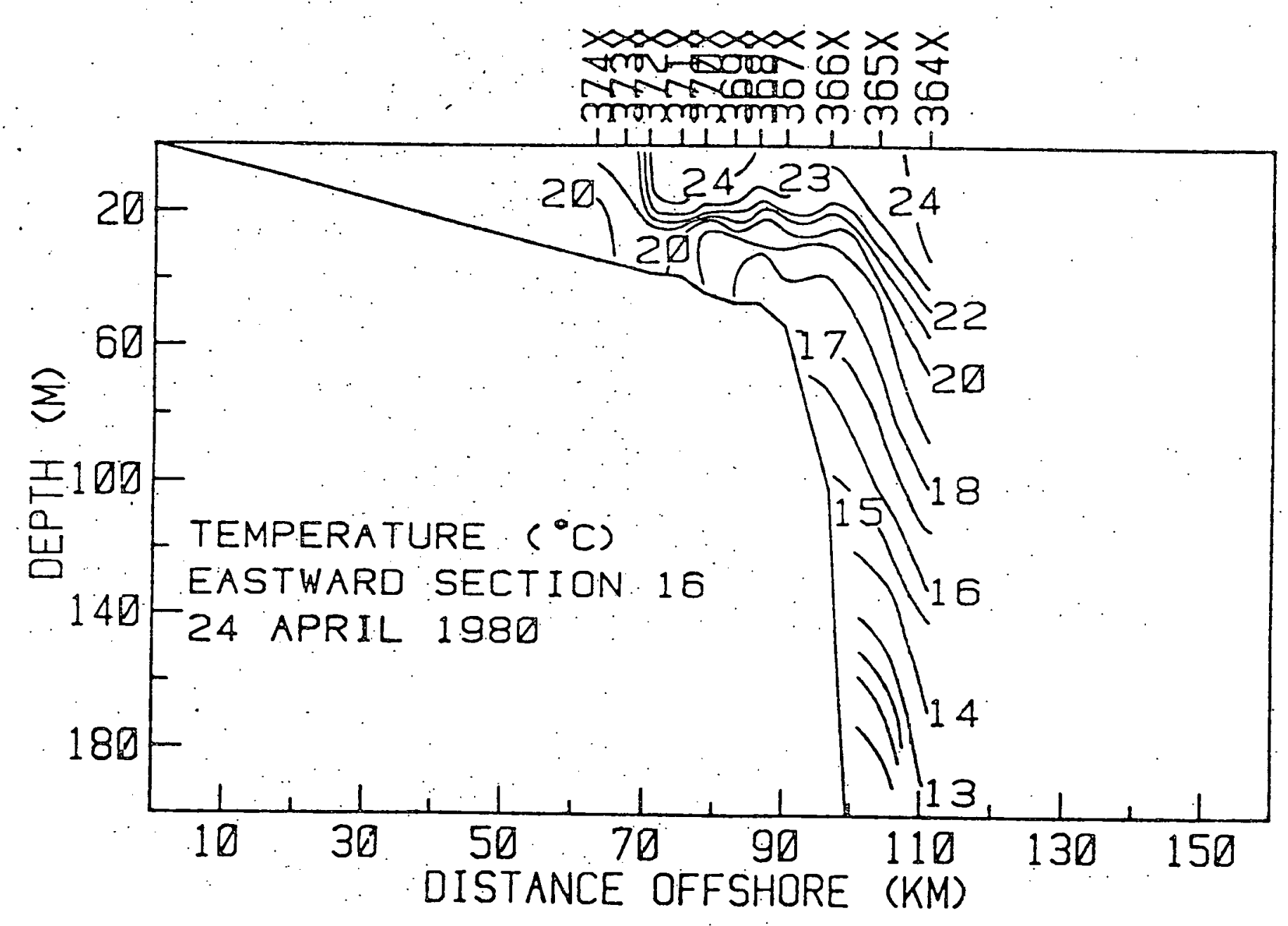




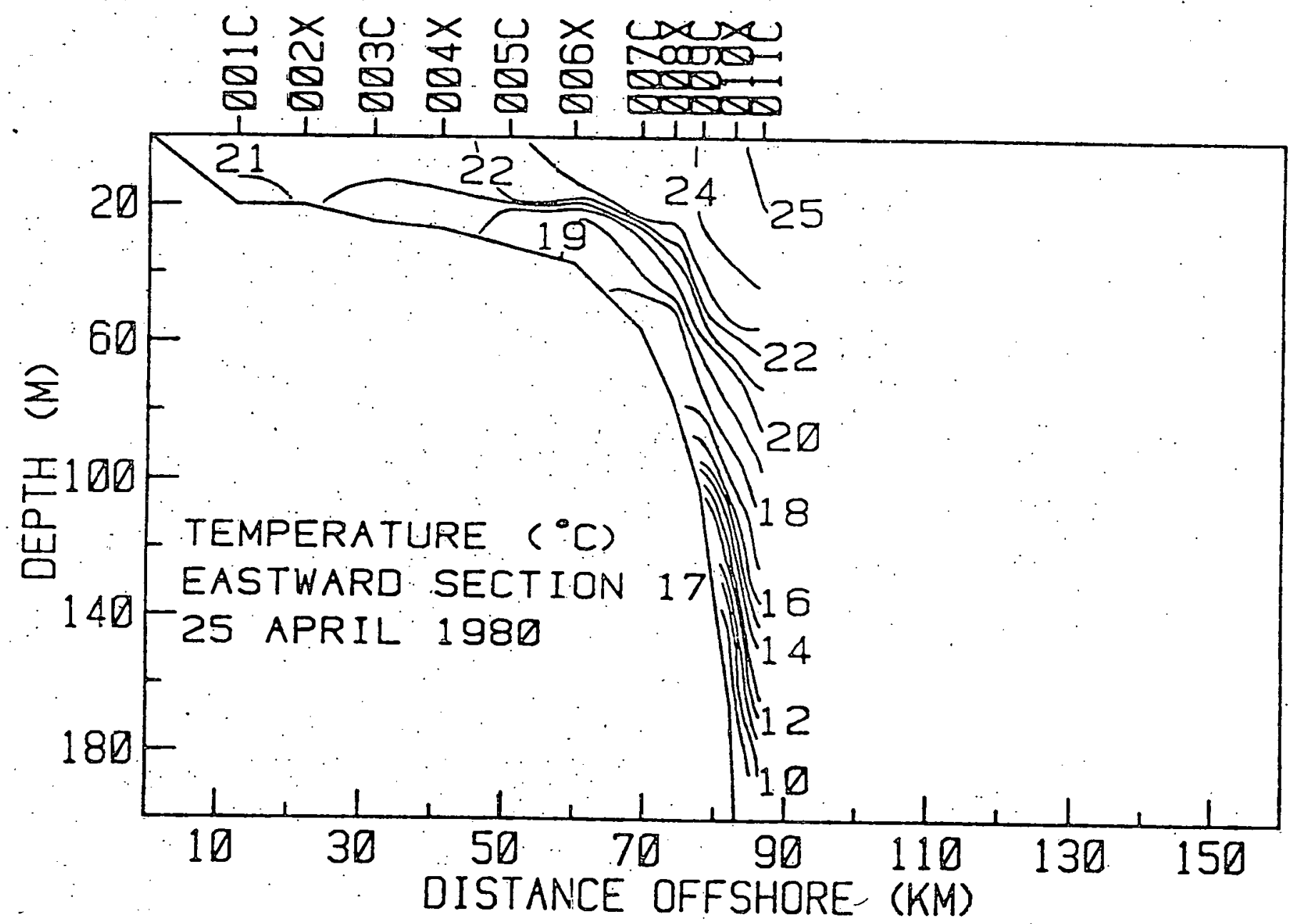




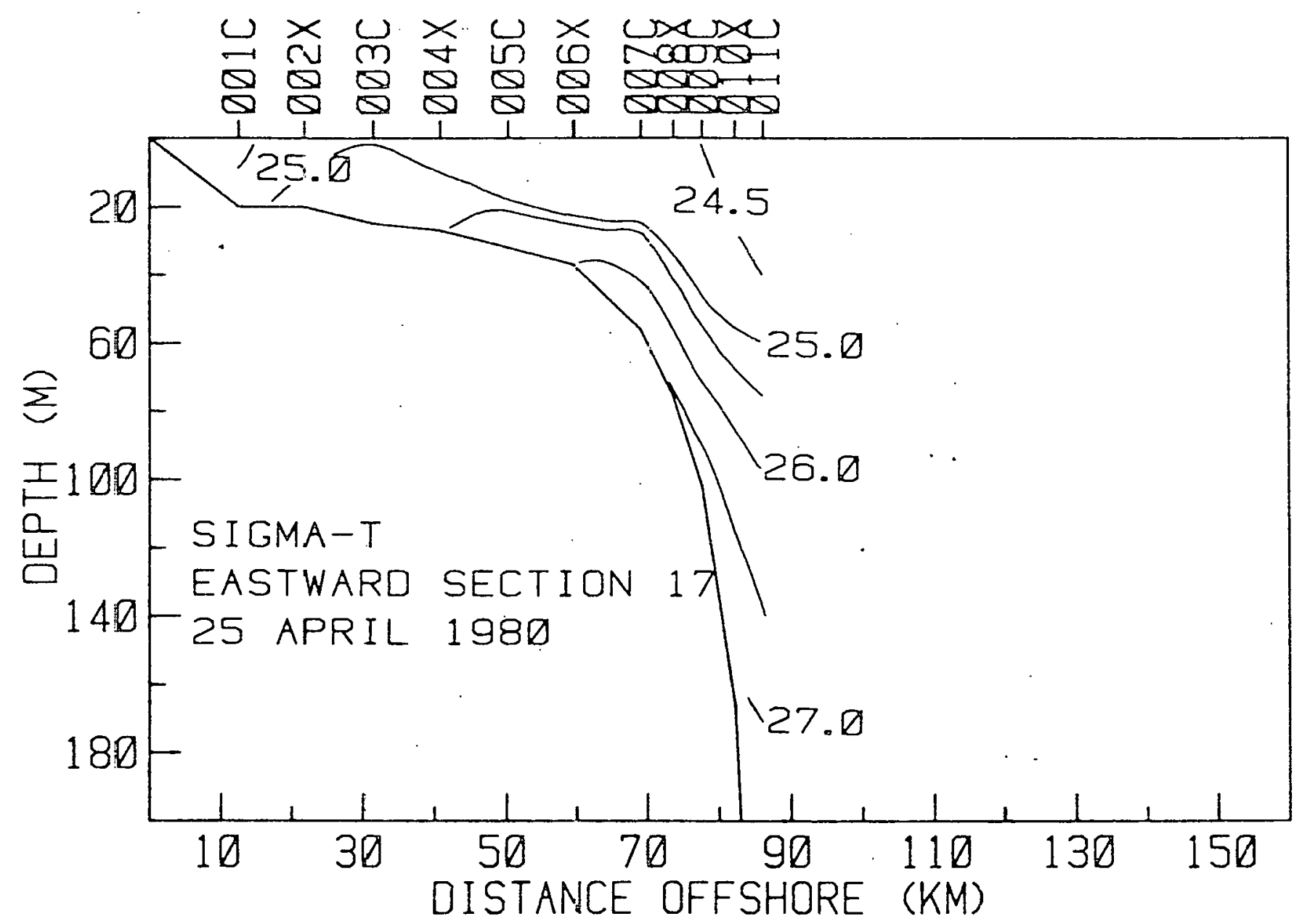




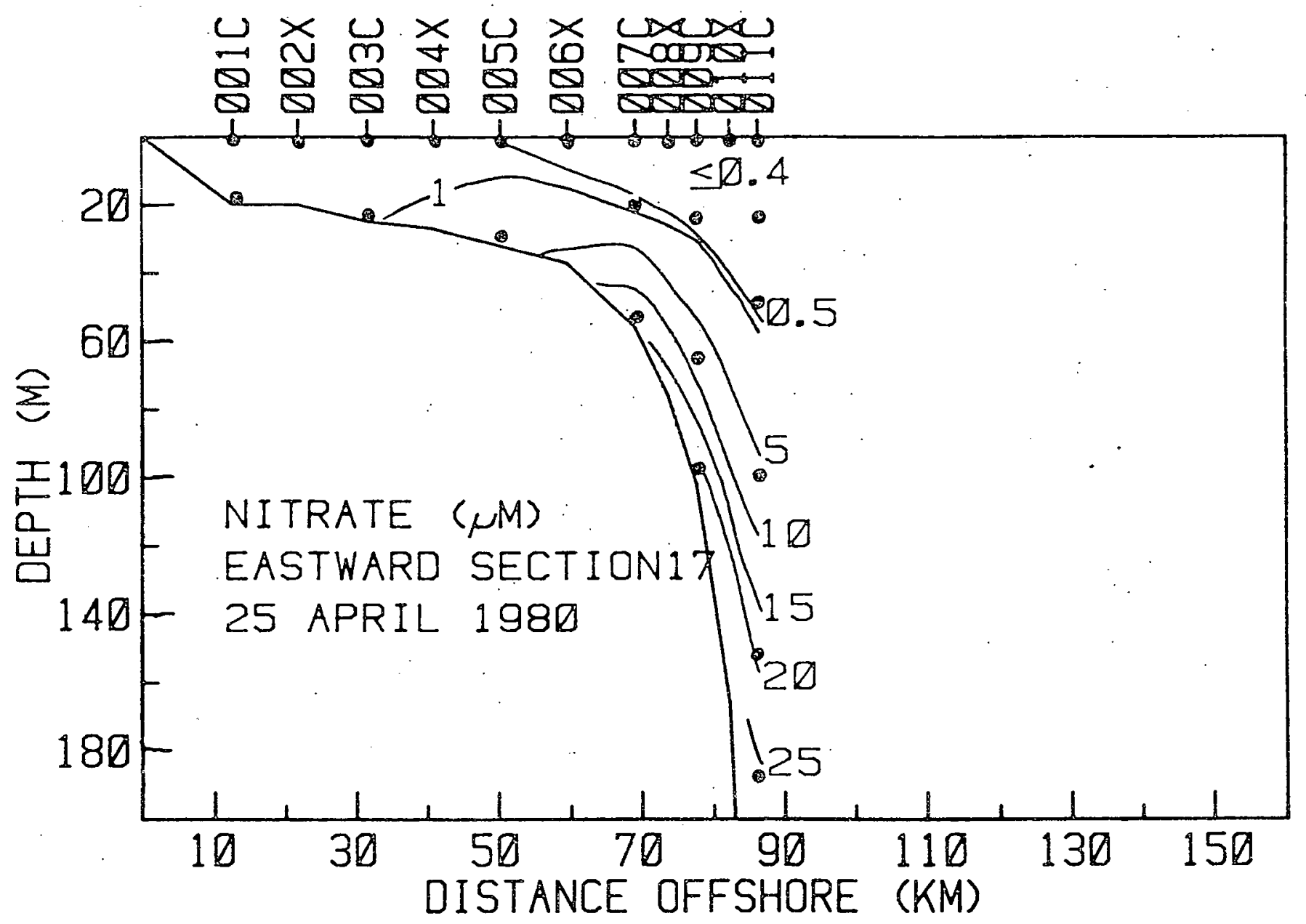



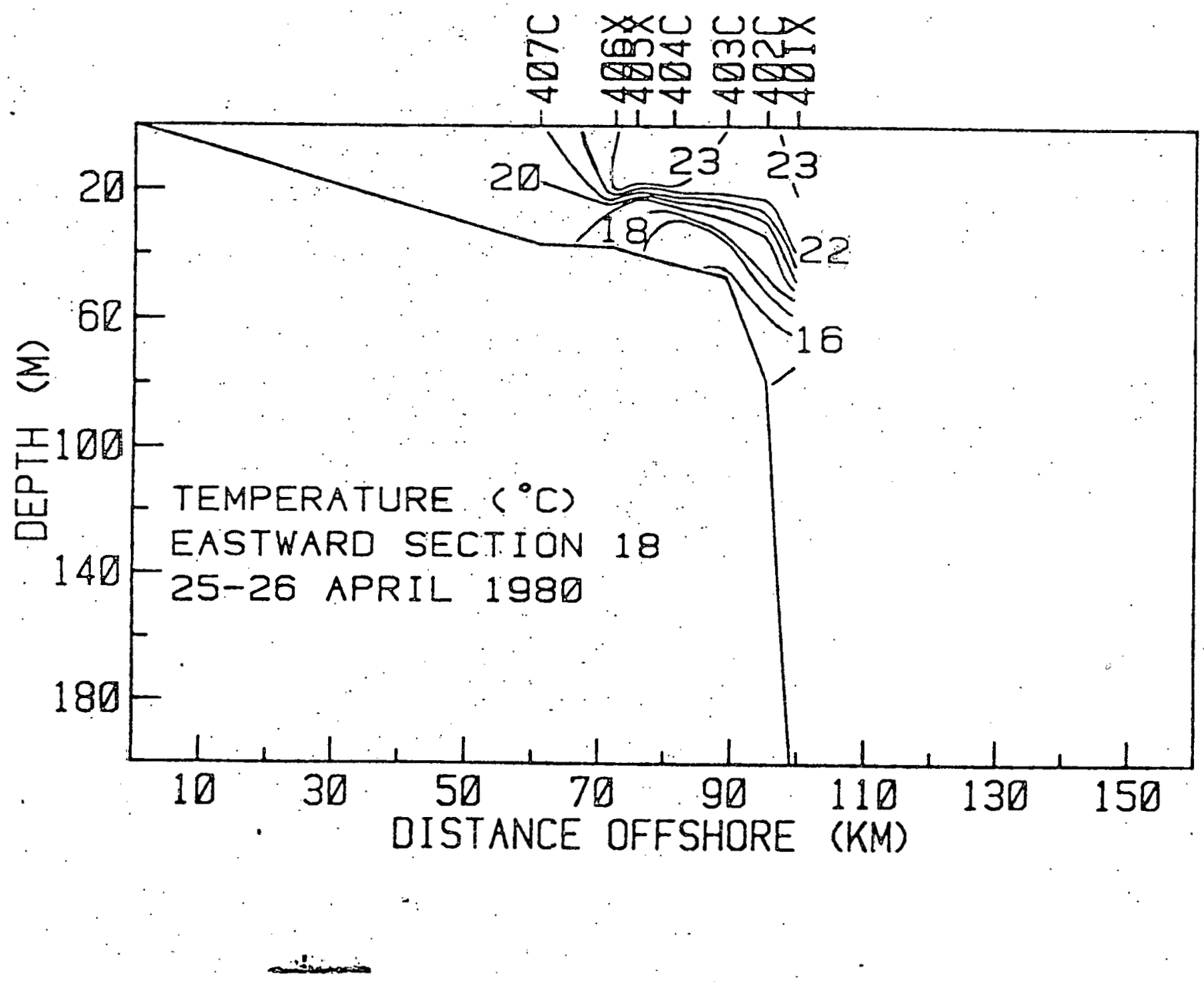


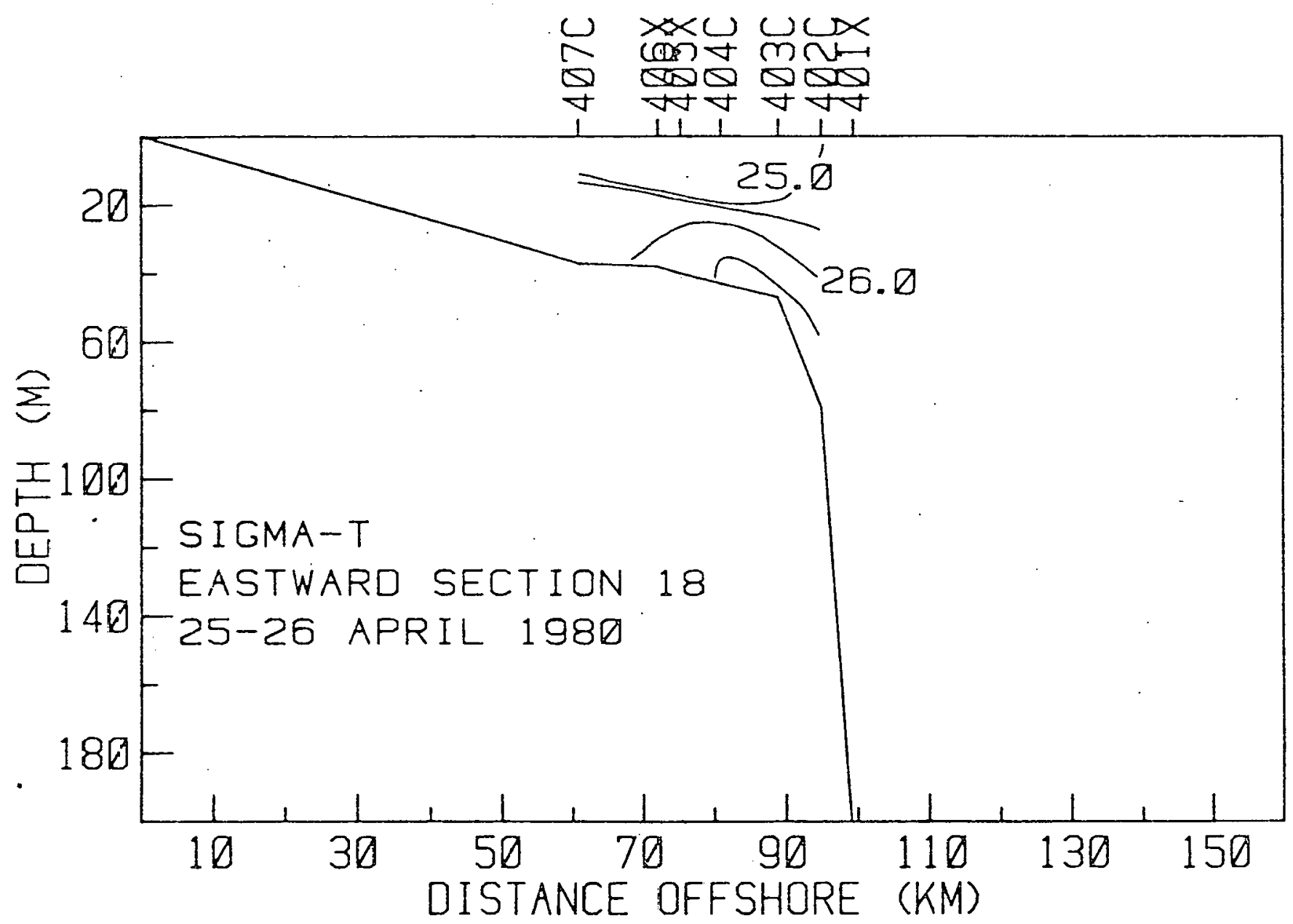




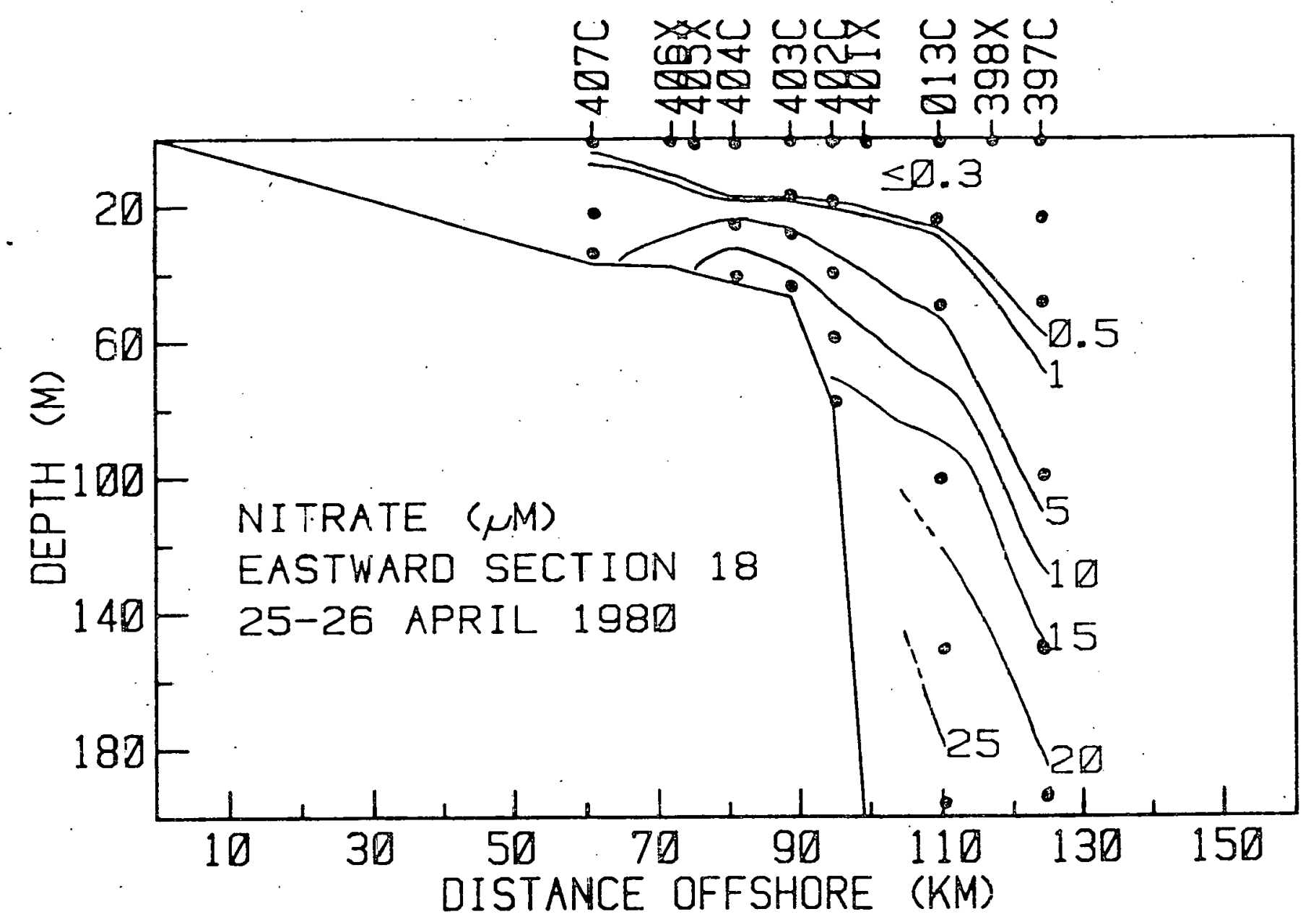




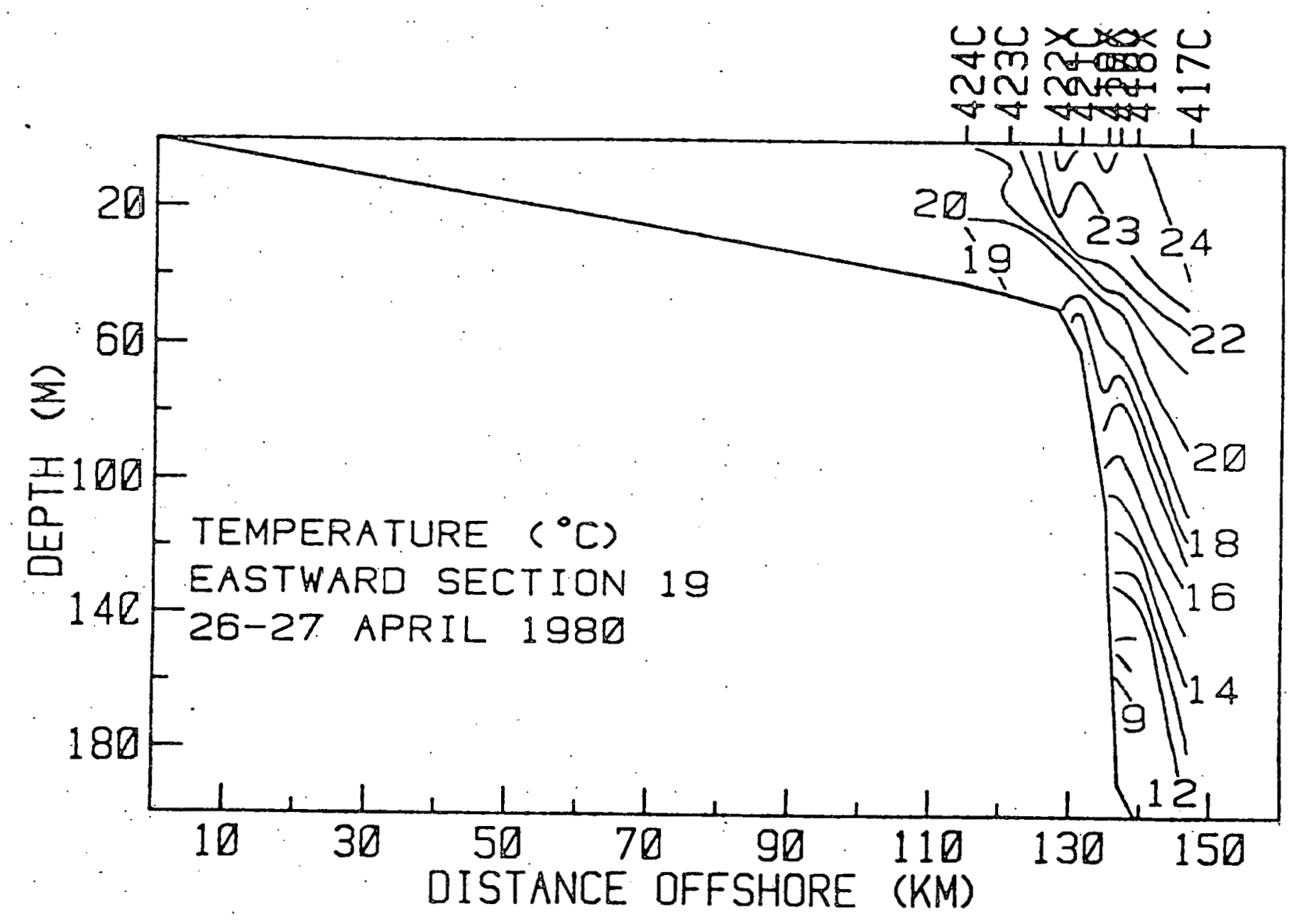




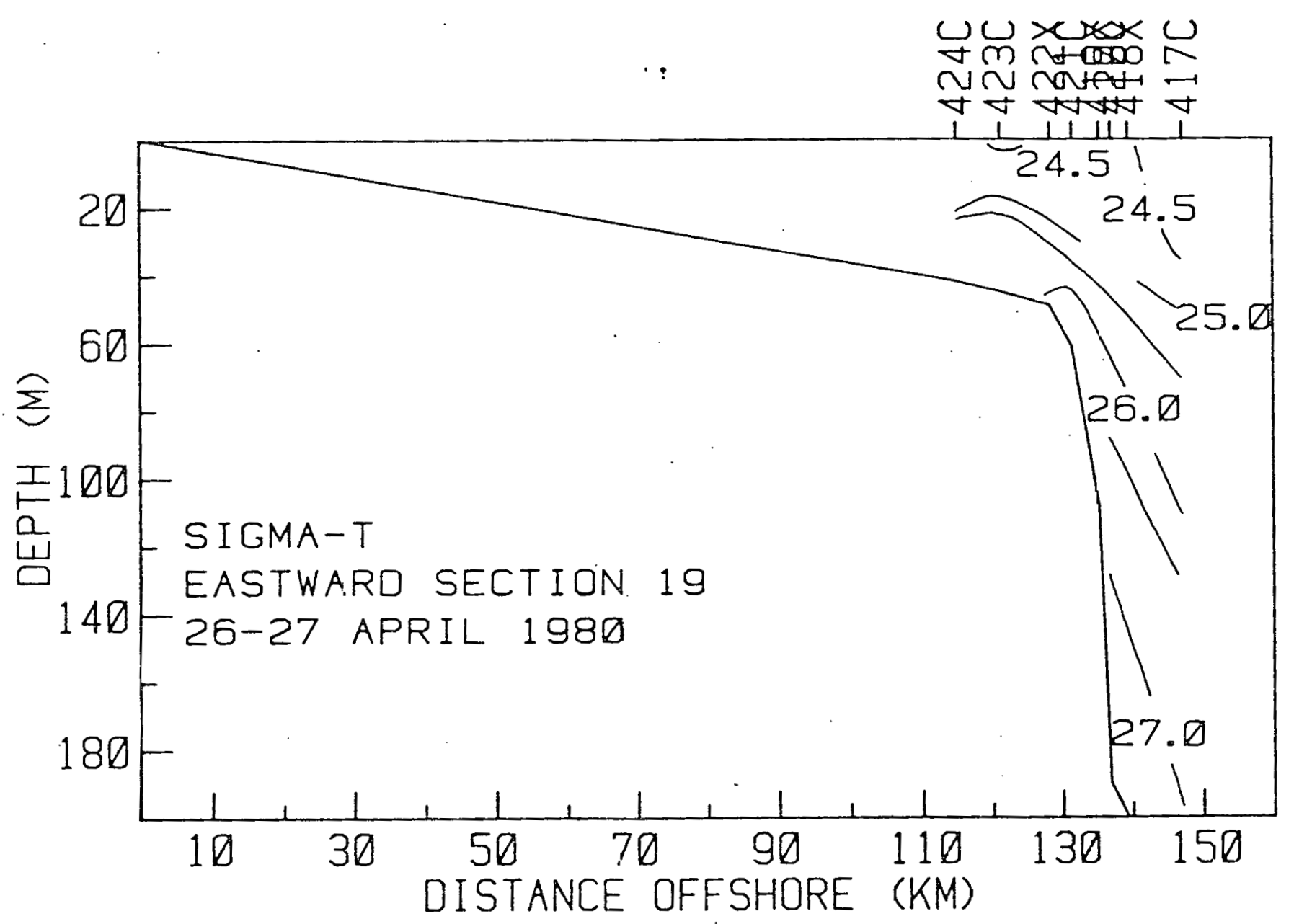




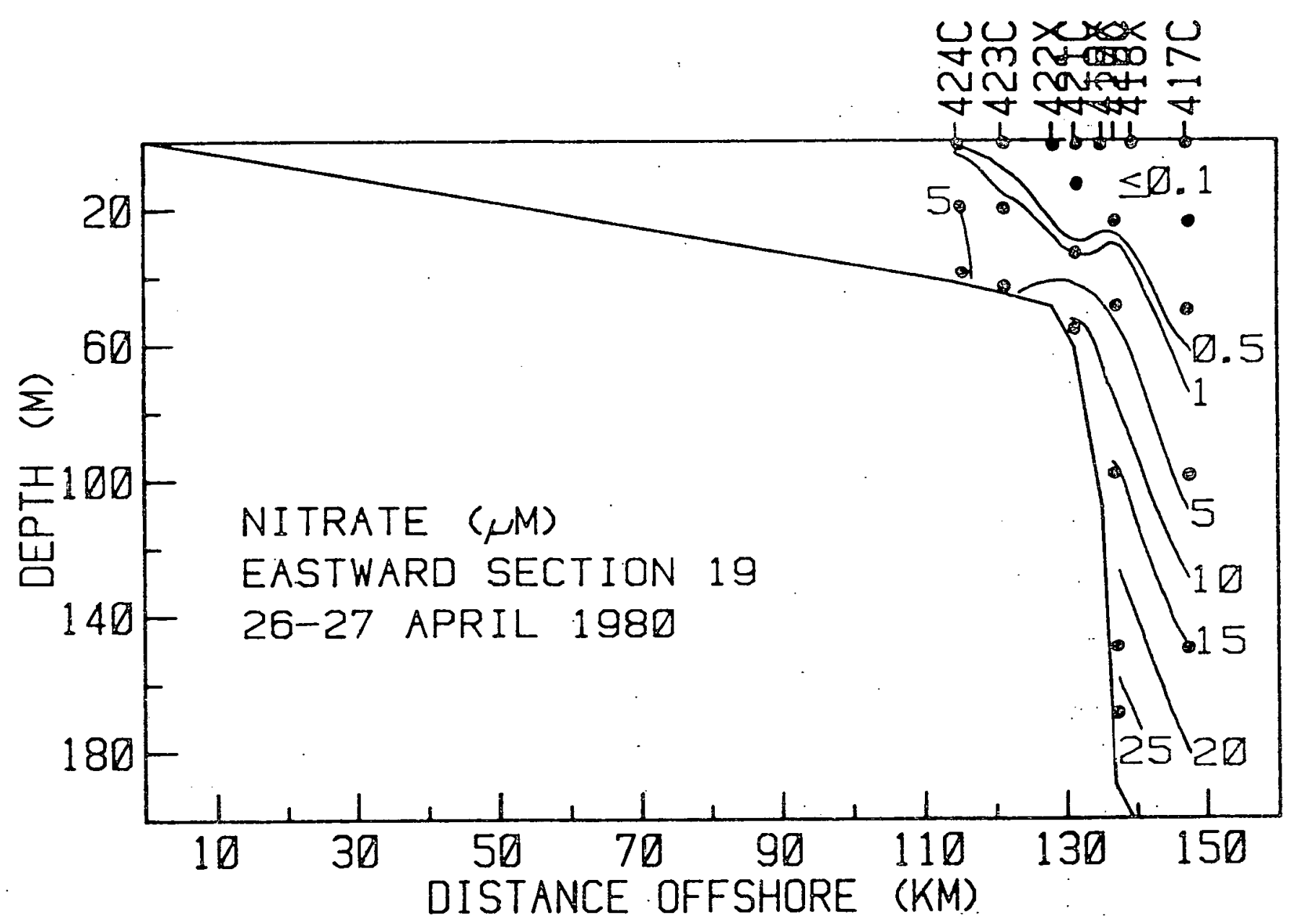




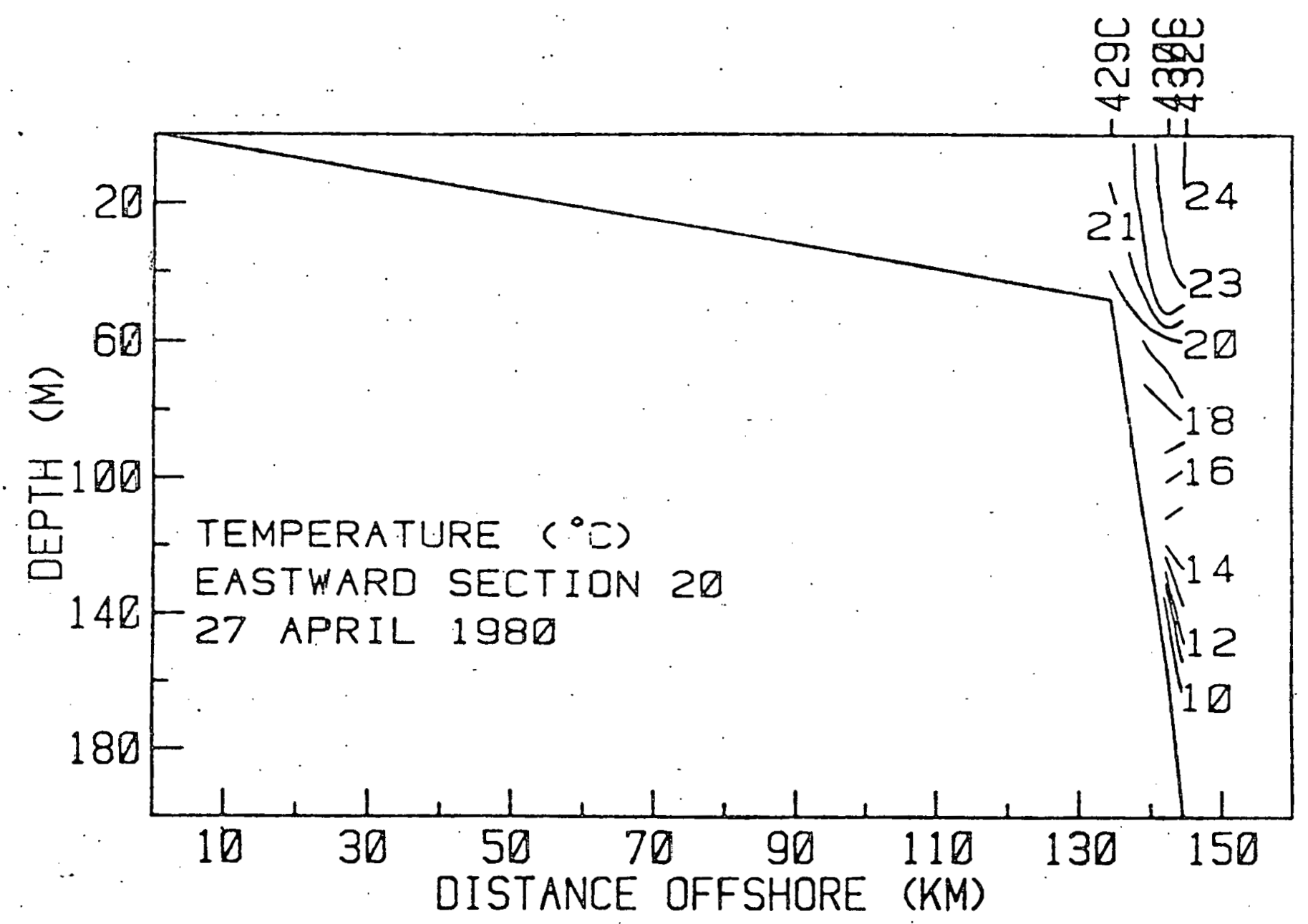

هذ- 


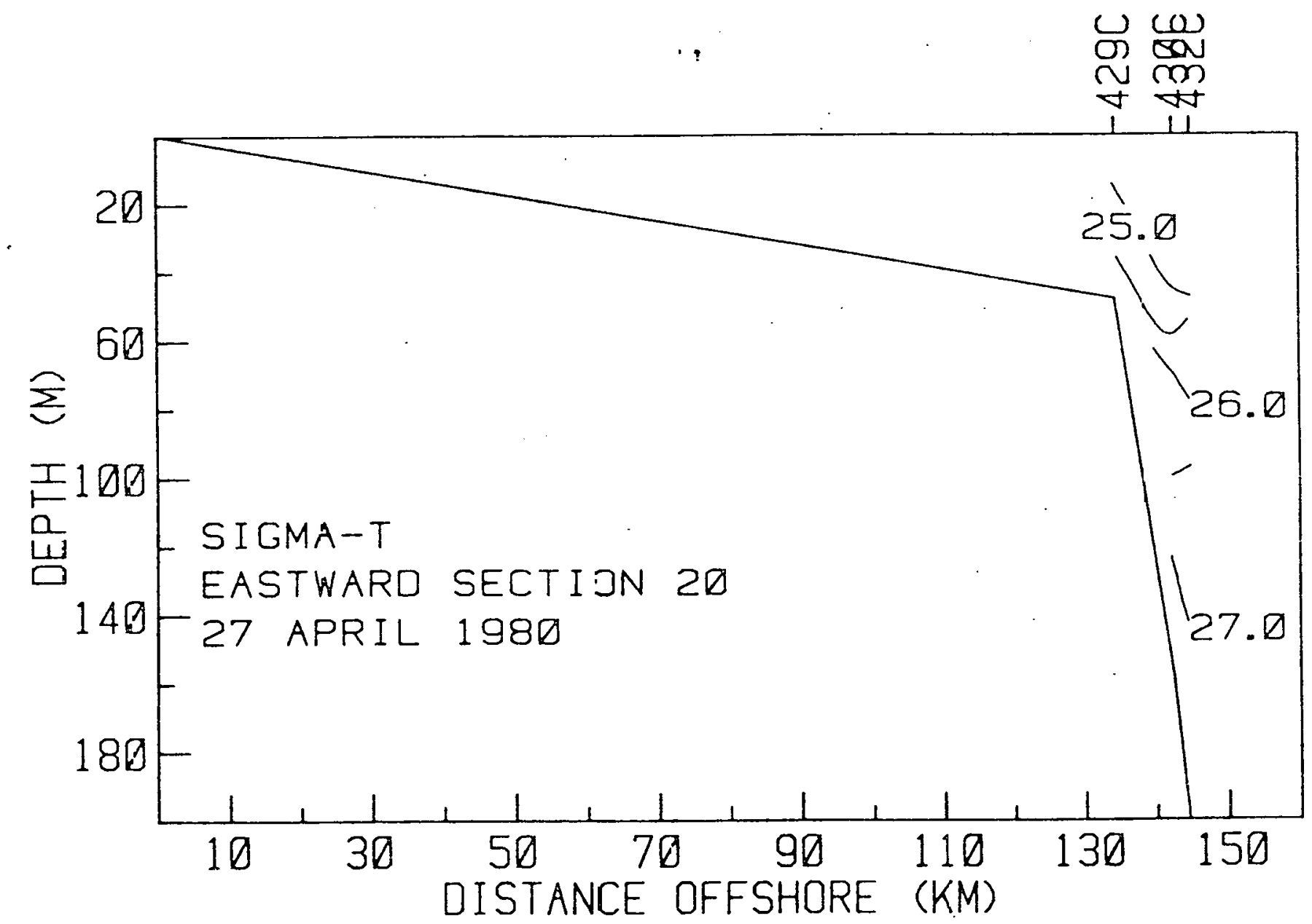




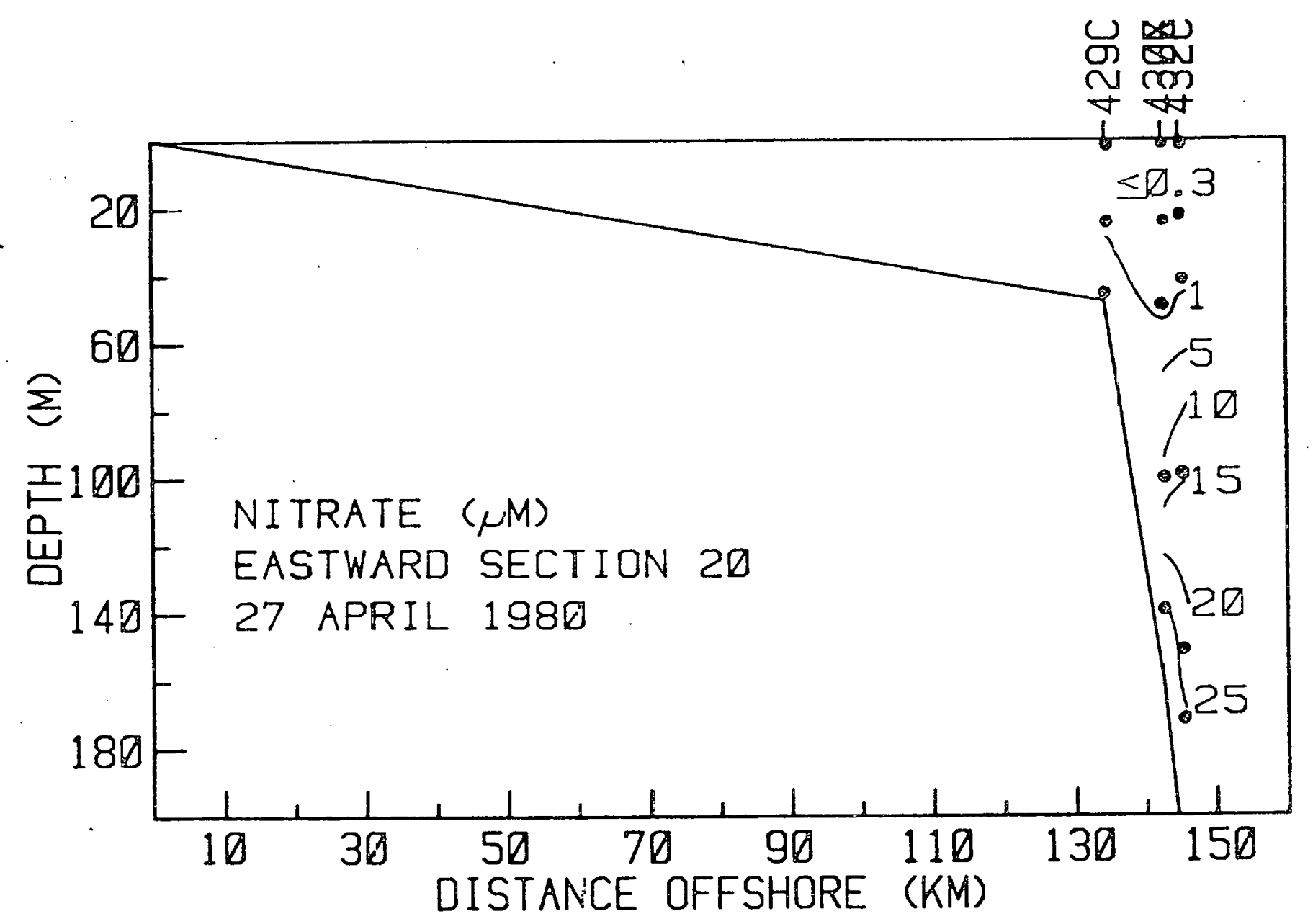




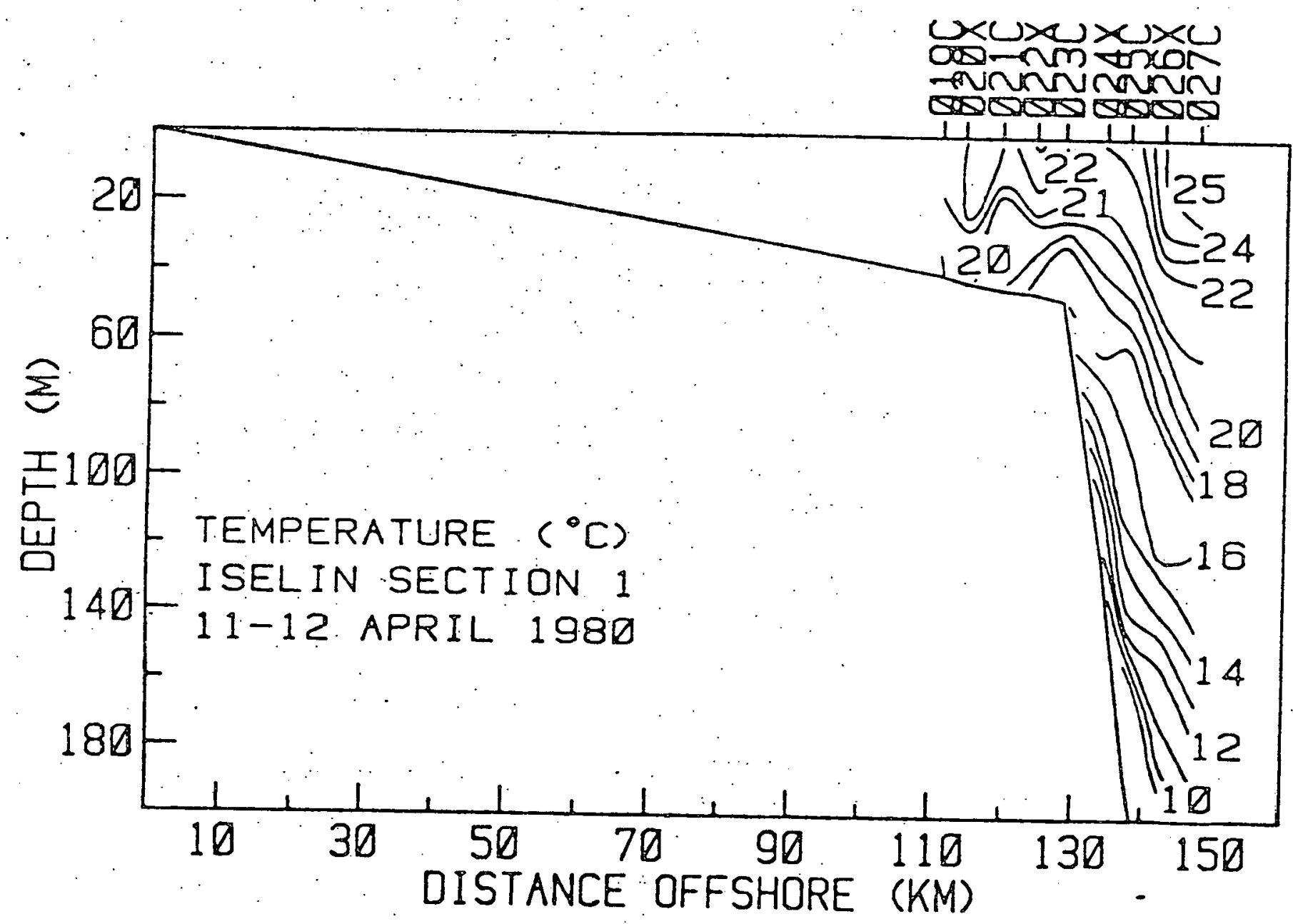




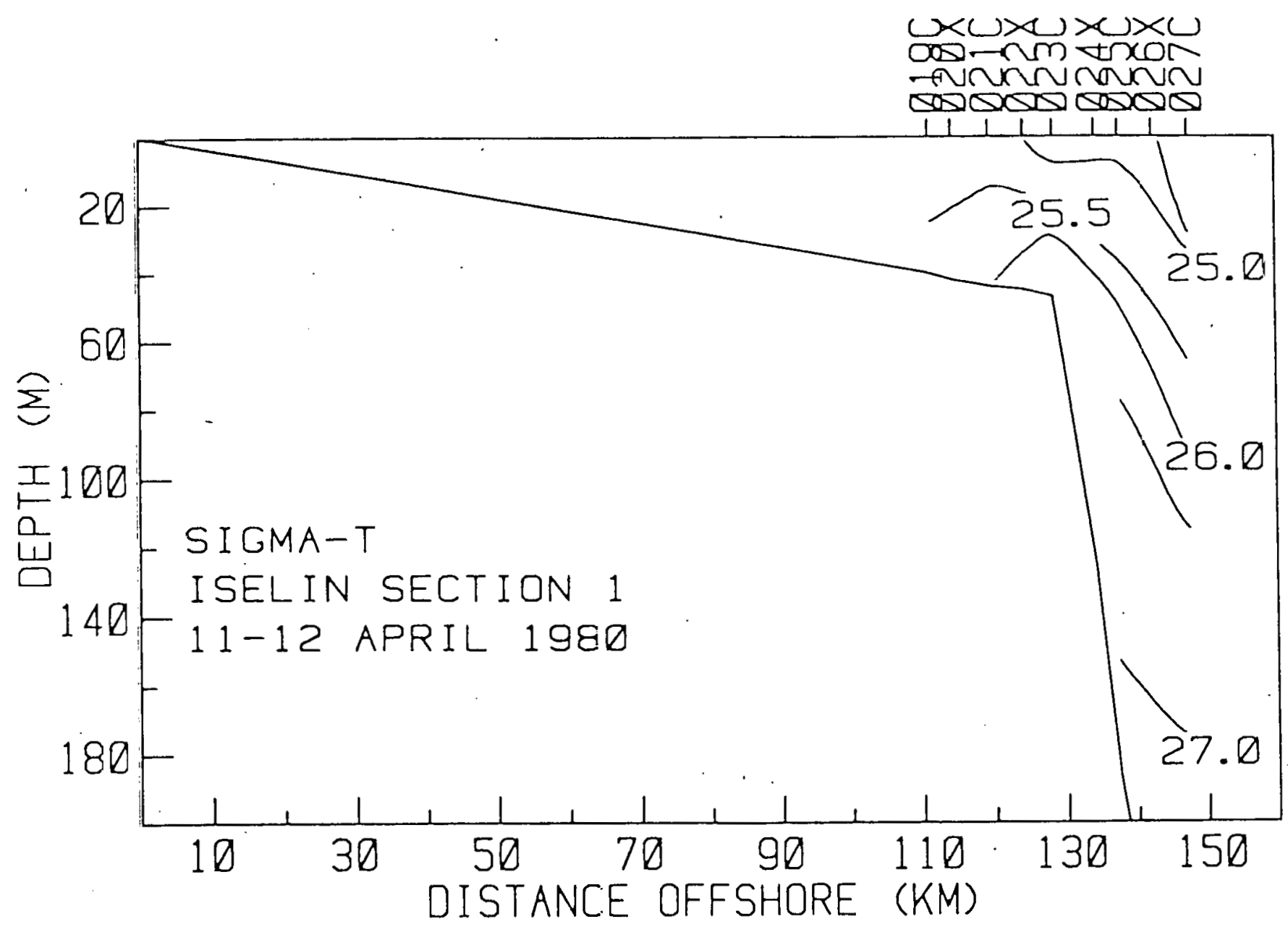



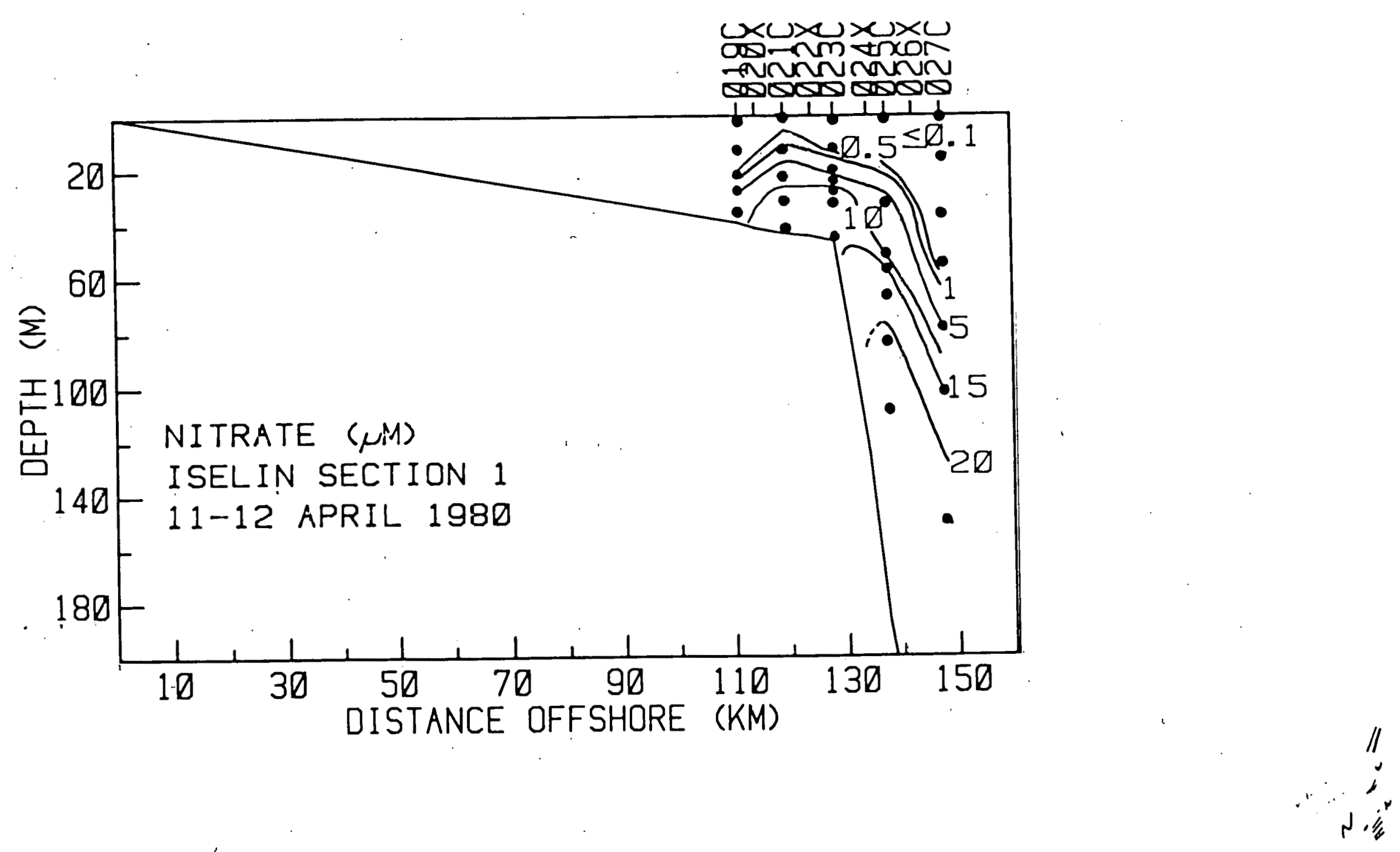


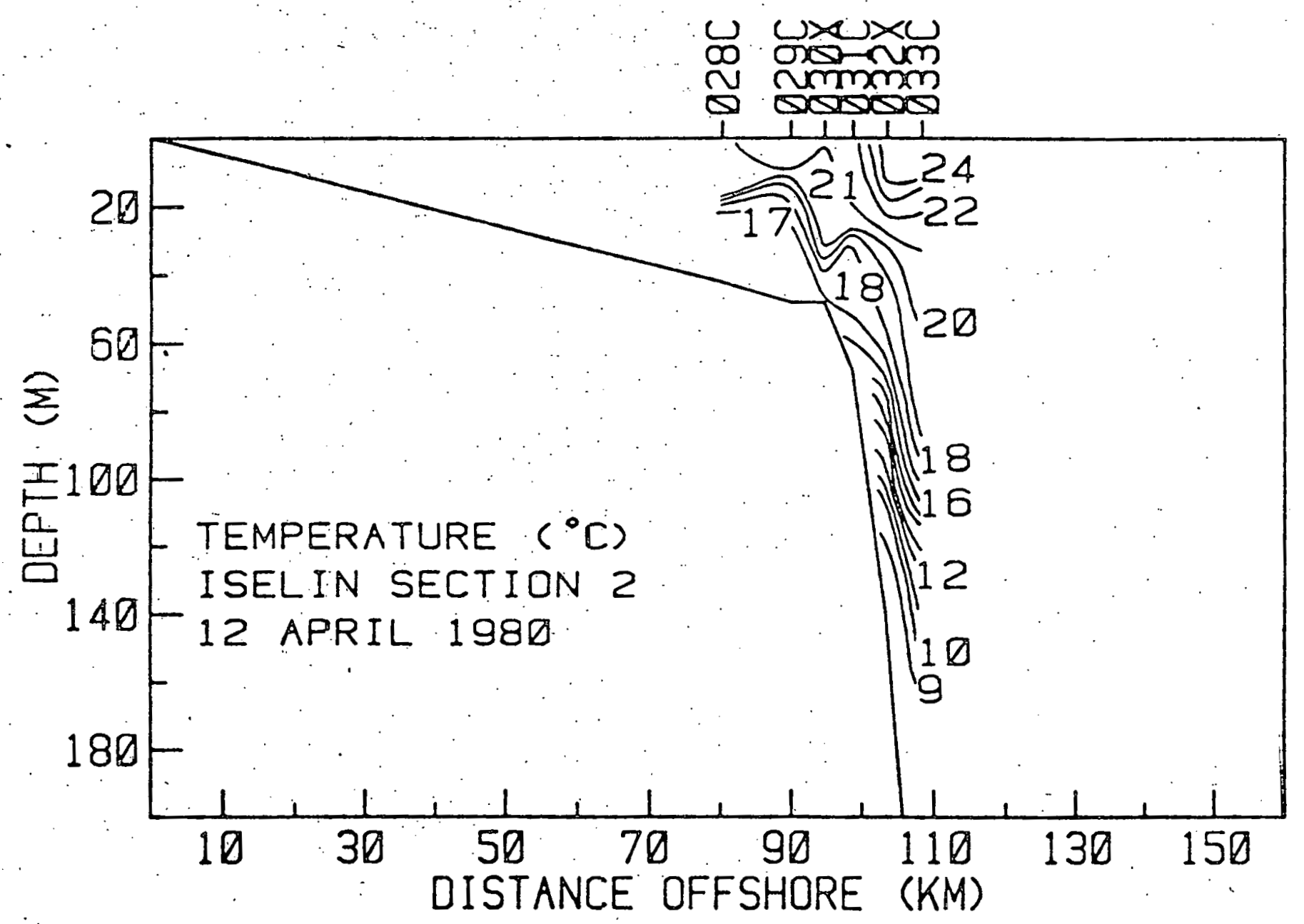




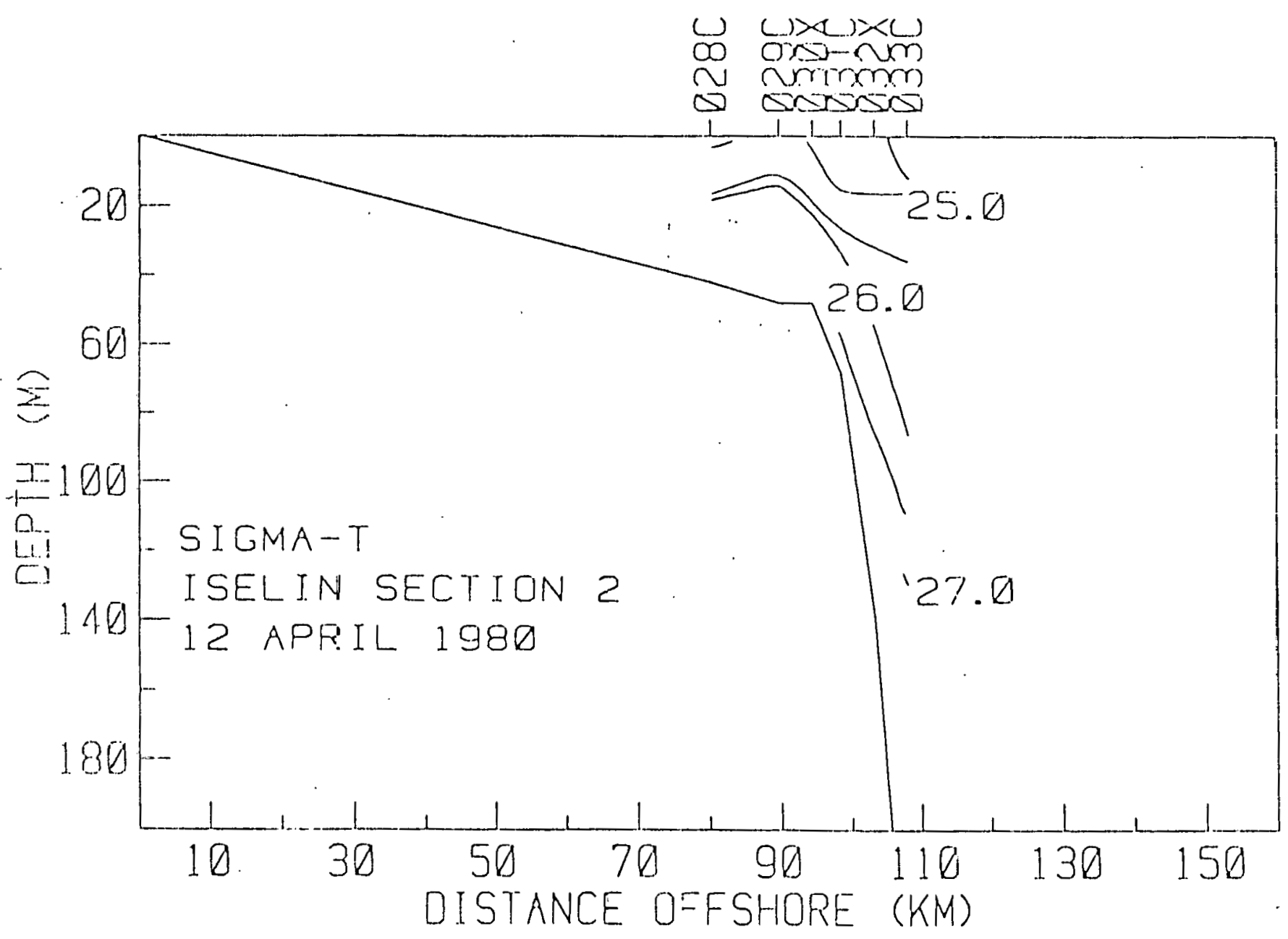




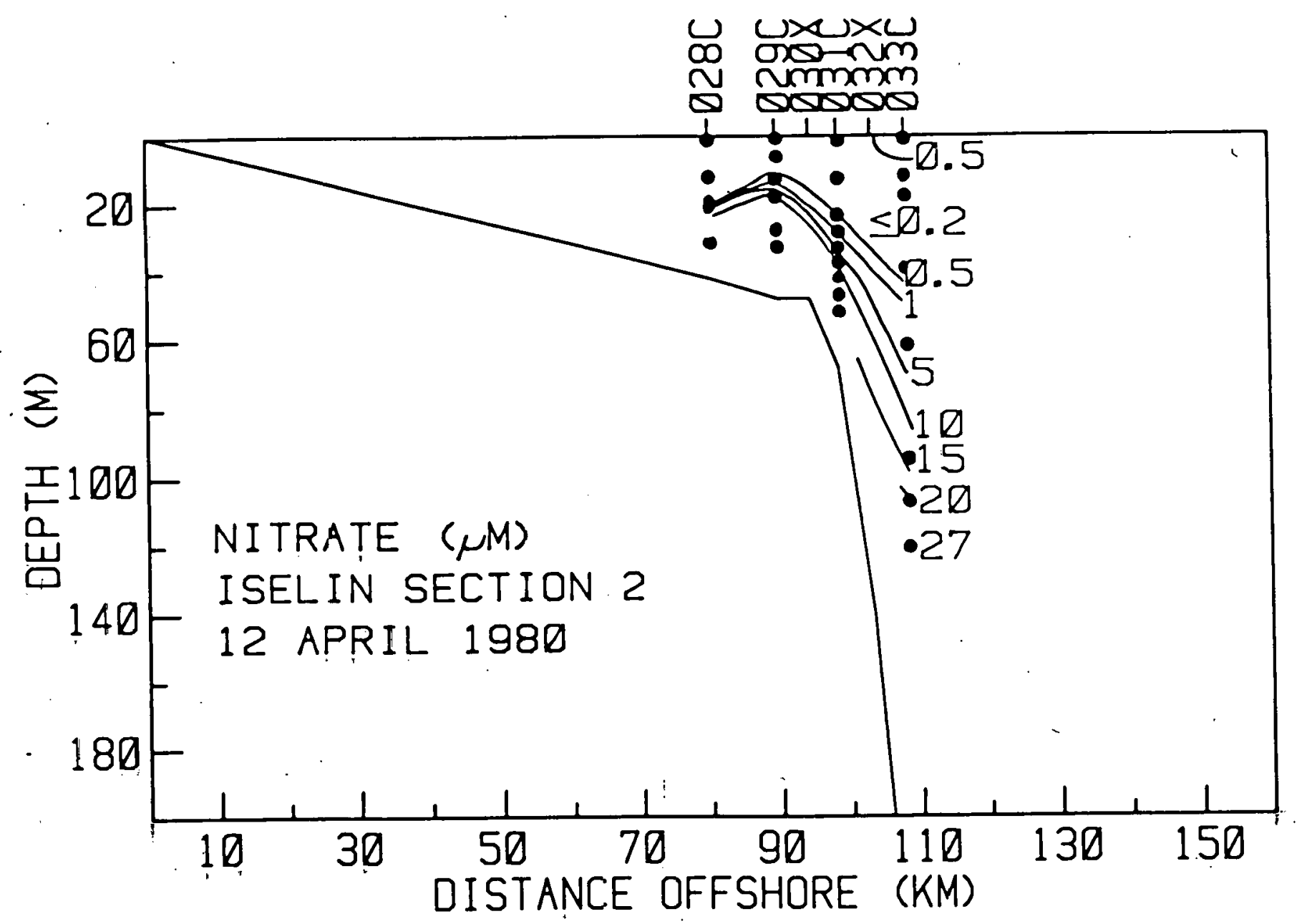




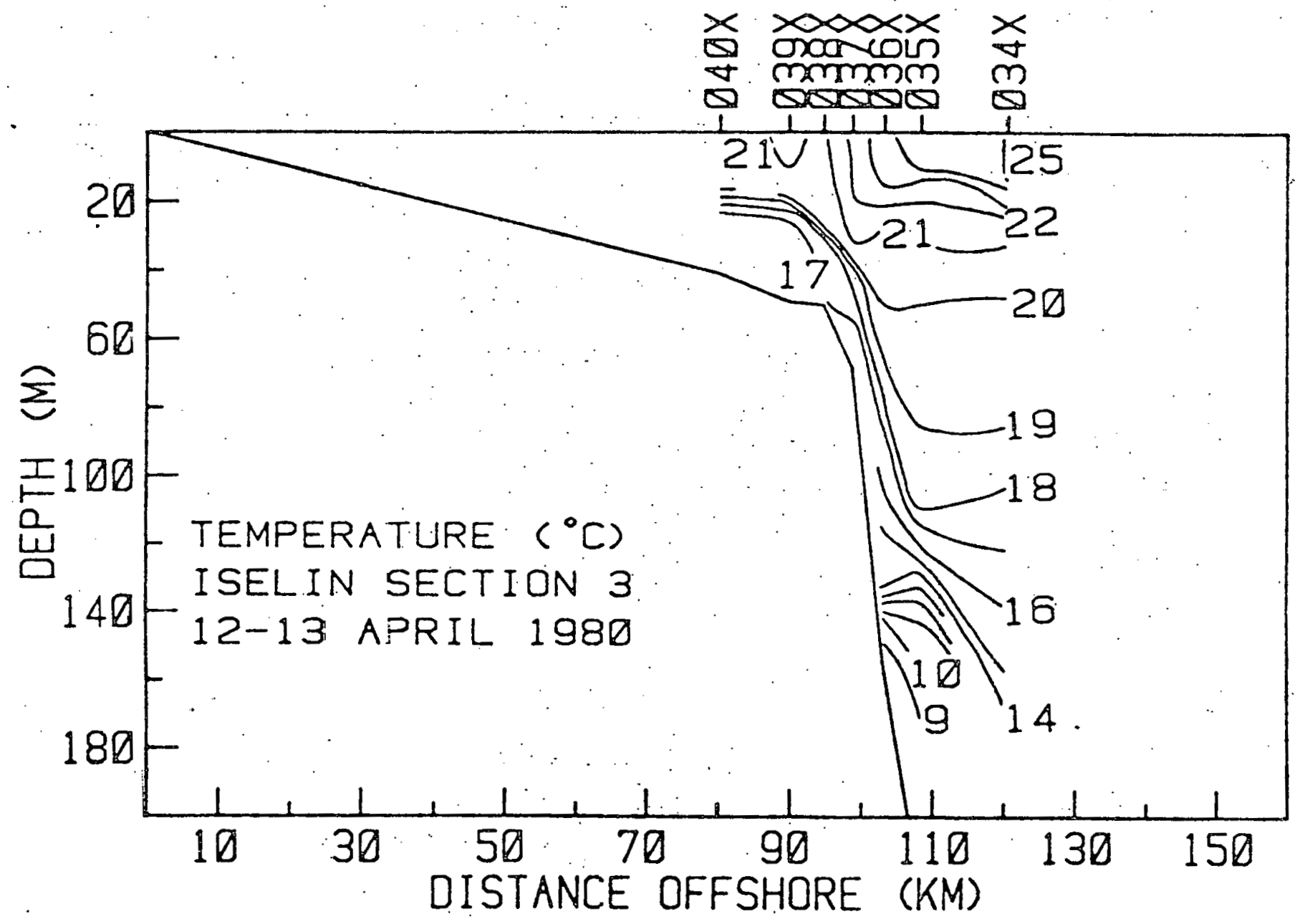




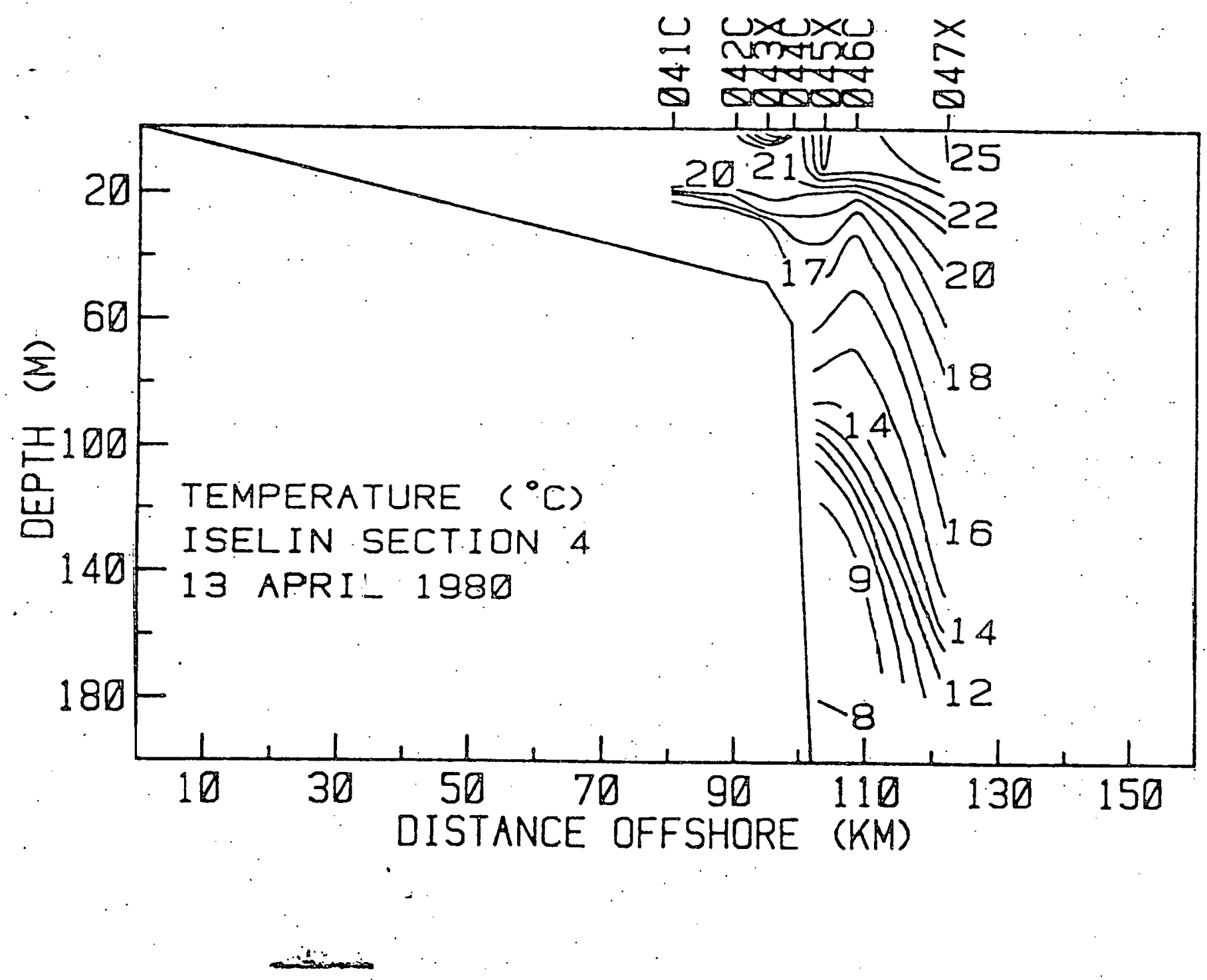




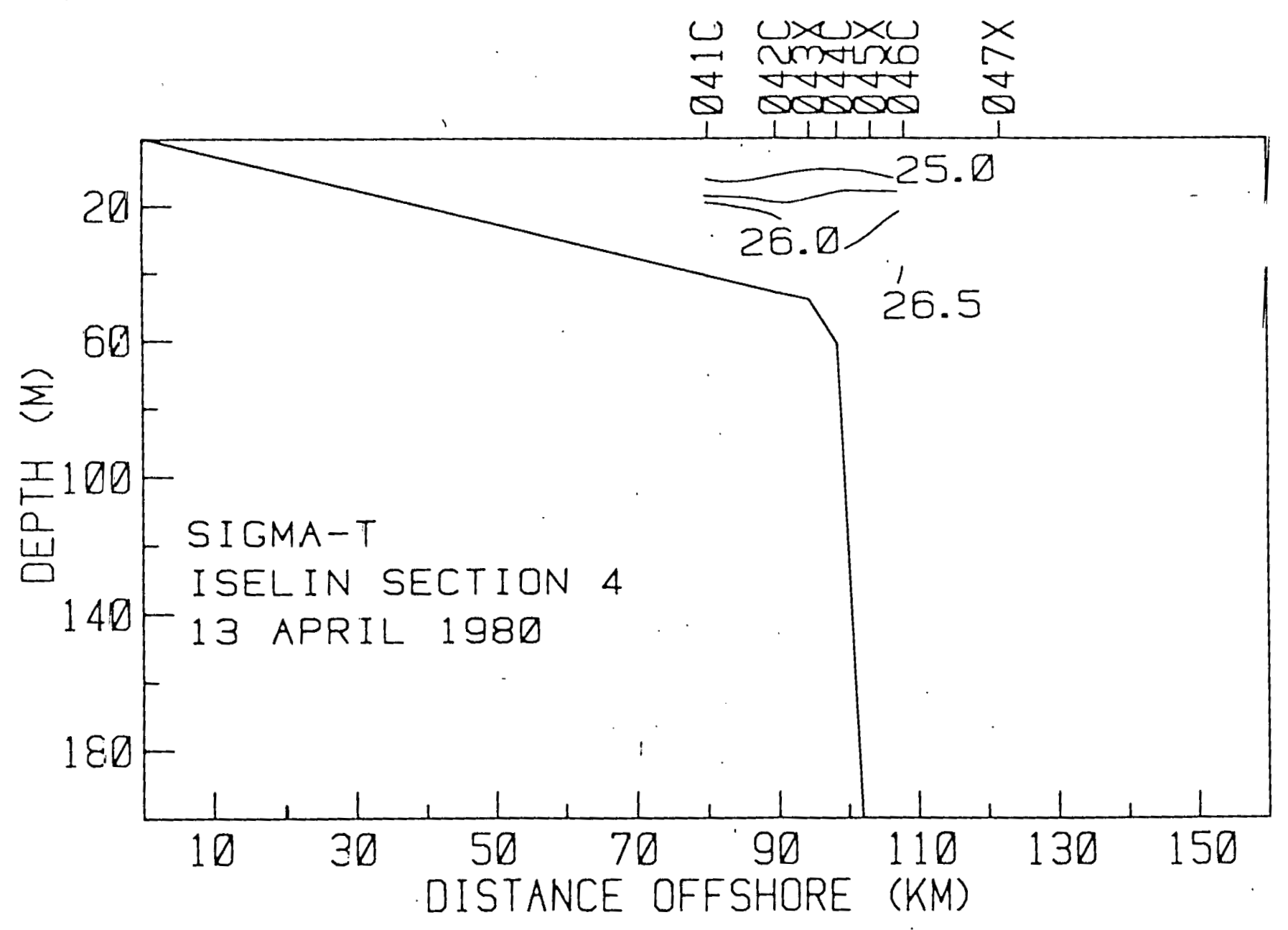




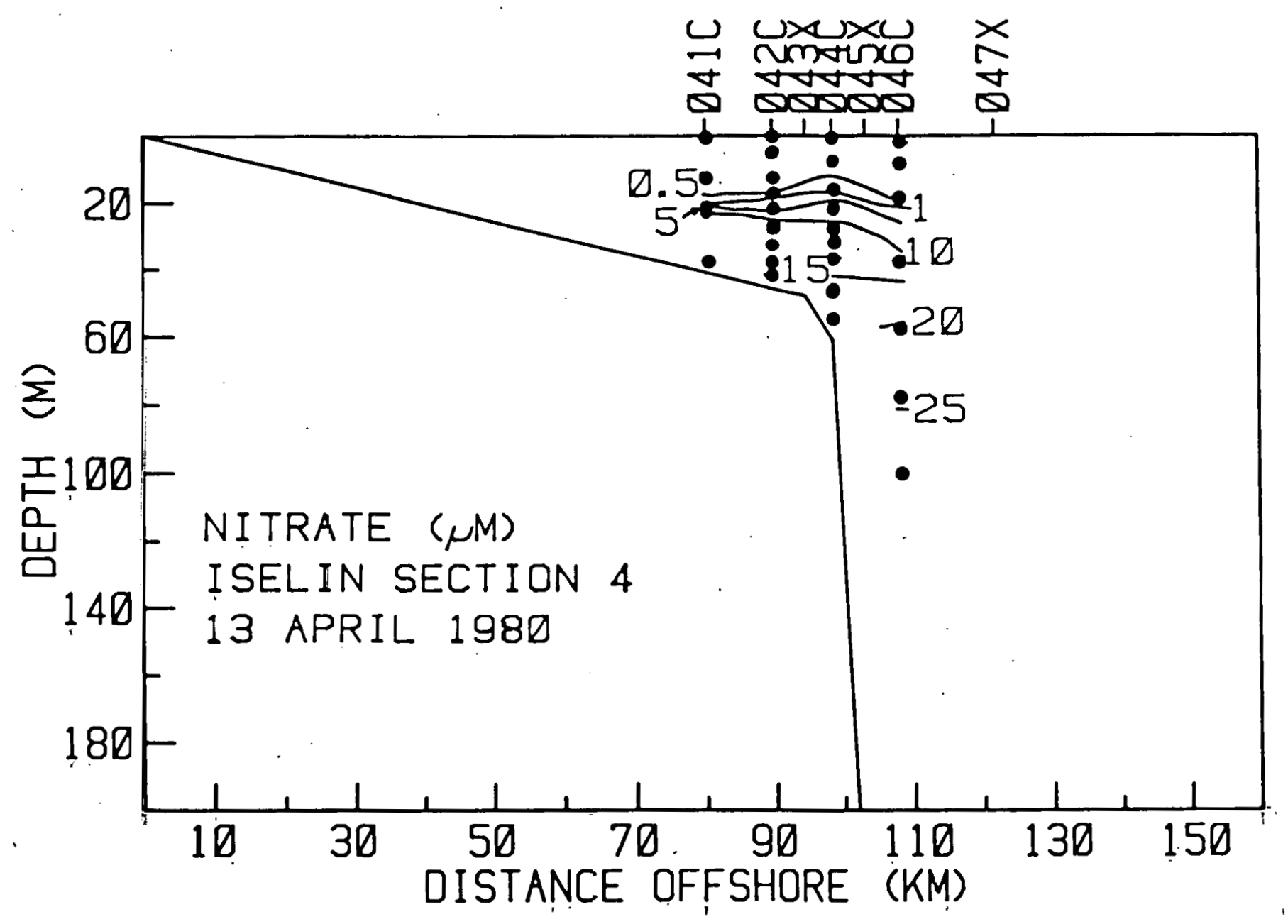




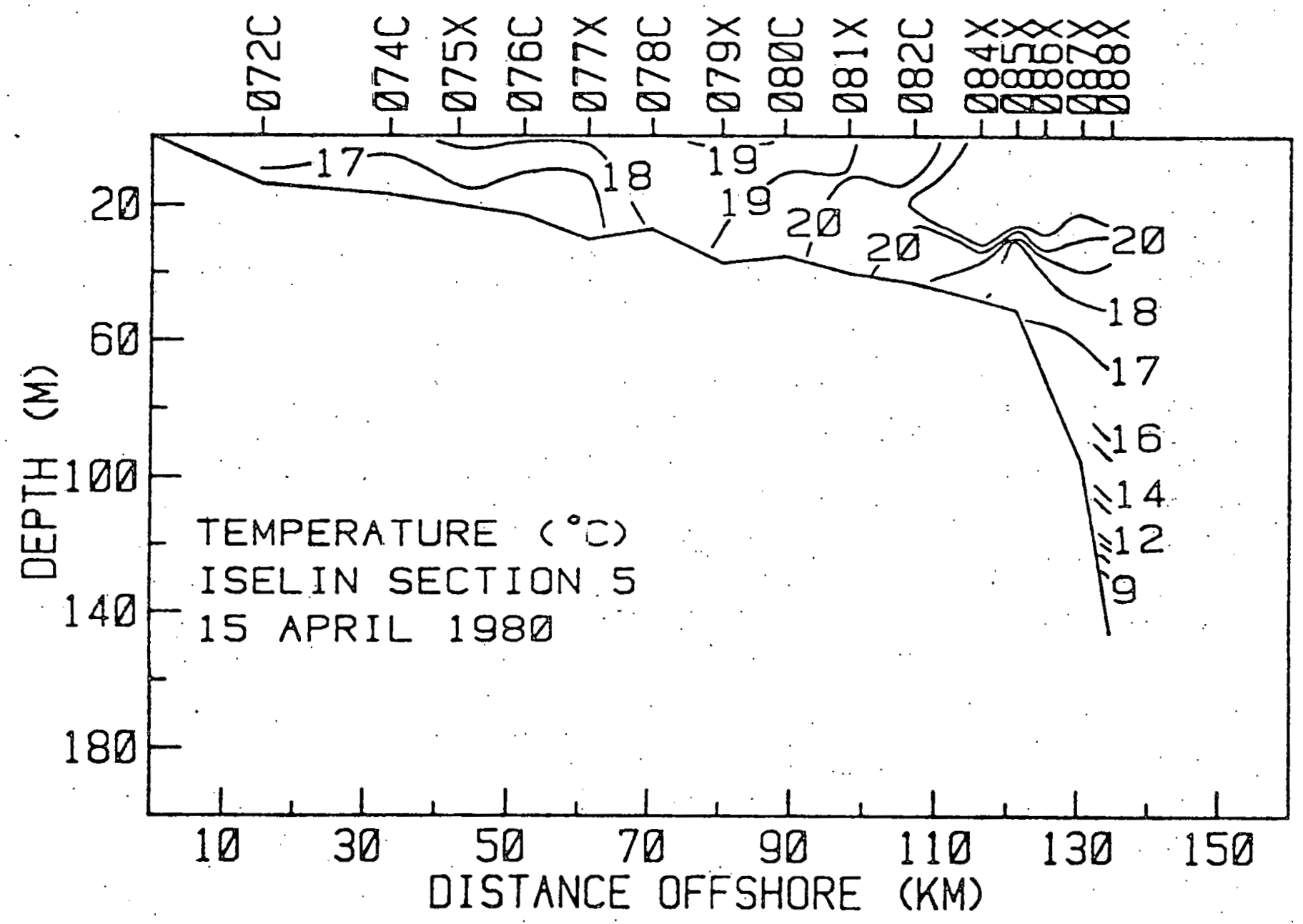




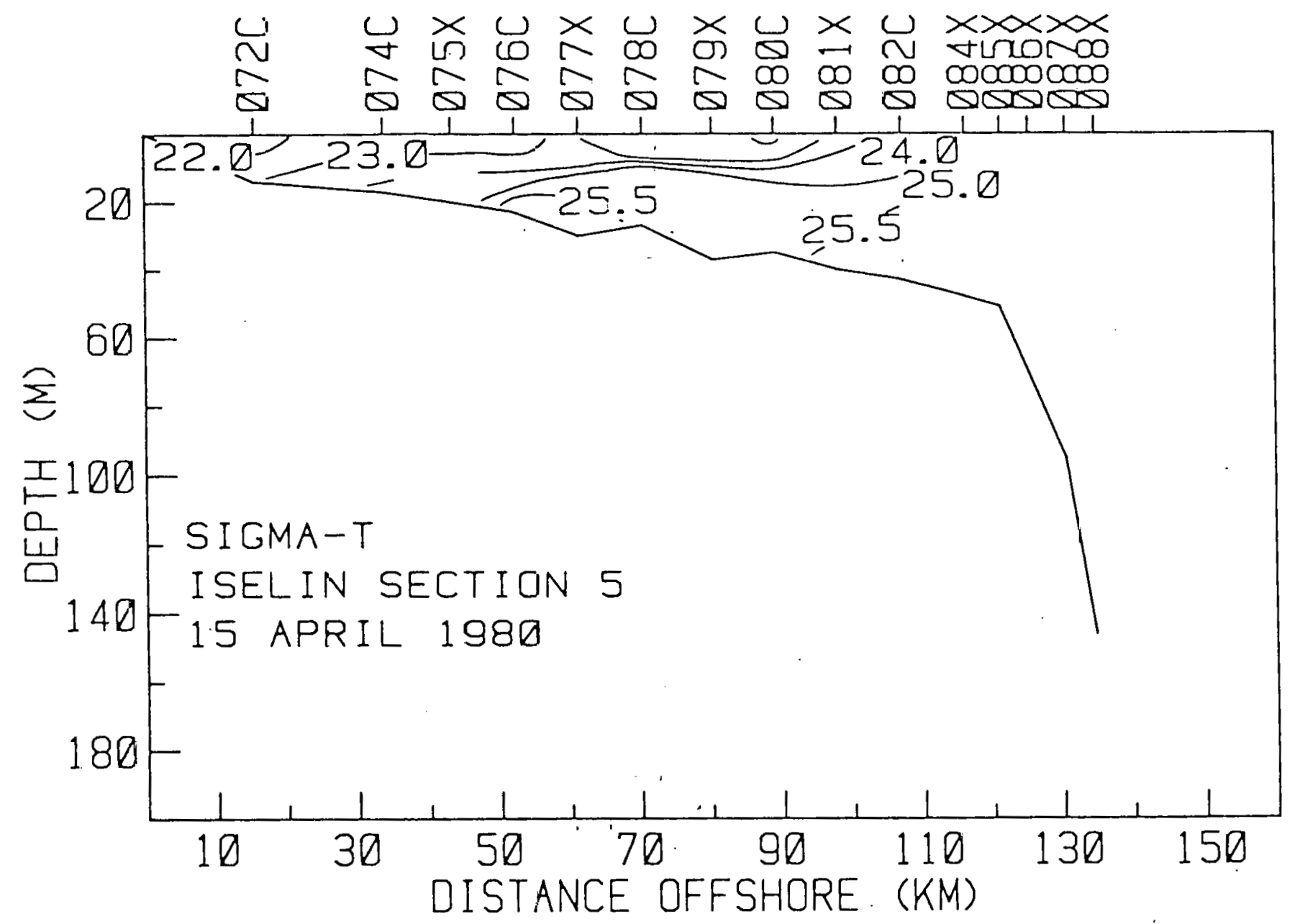




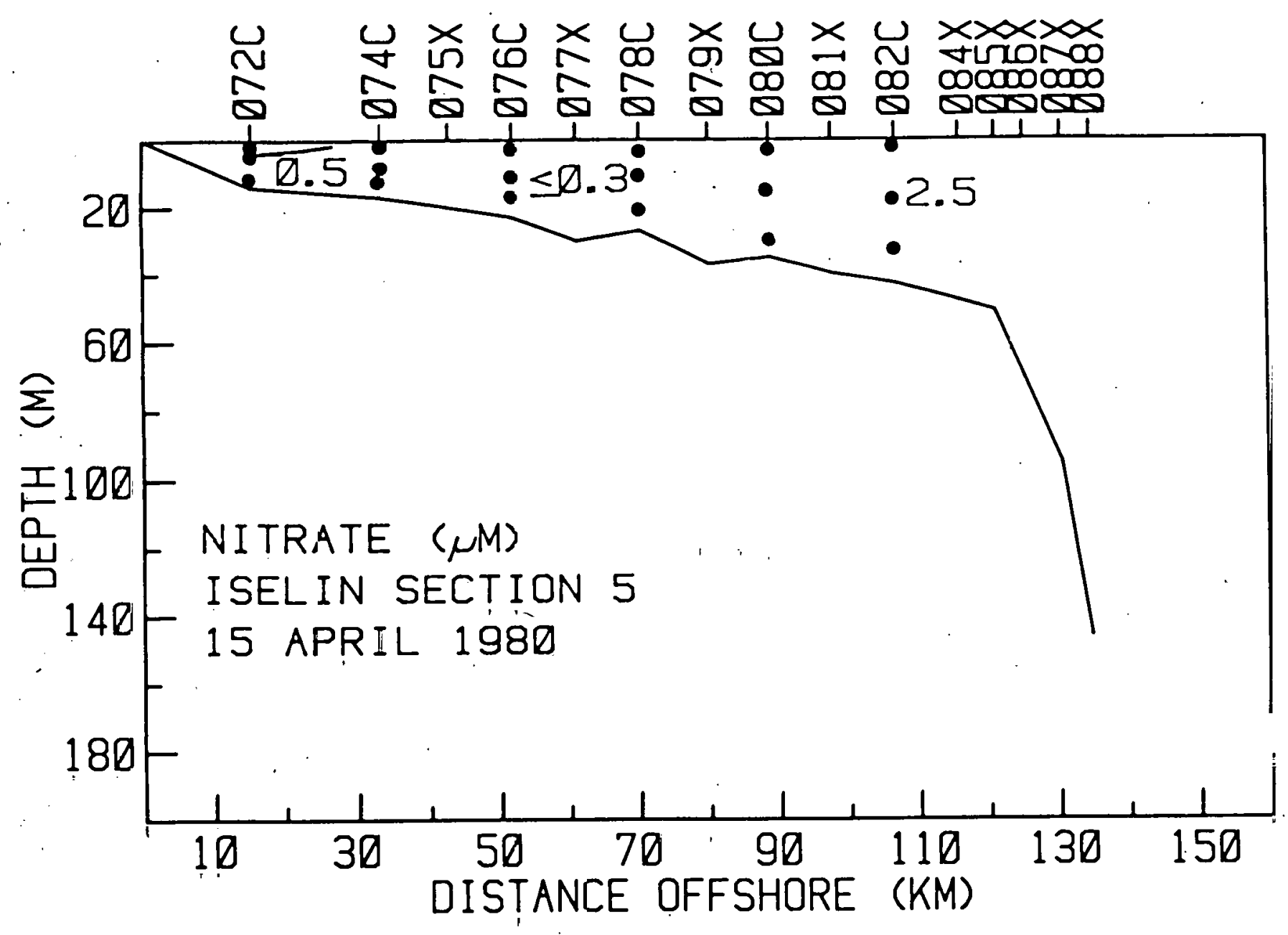




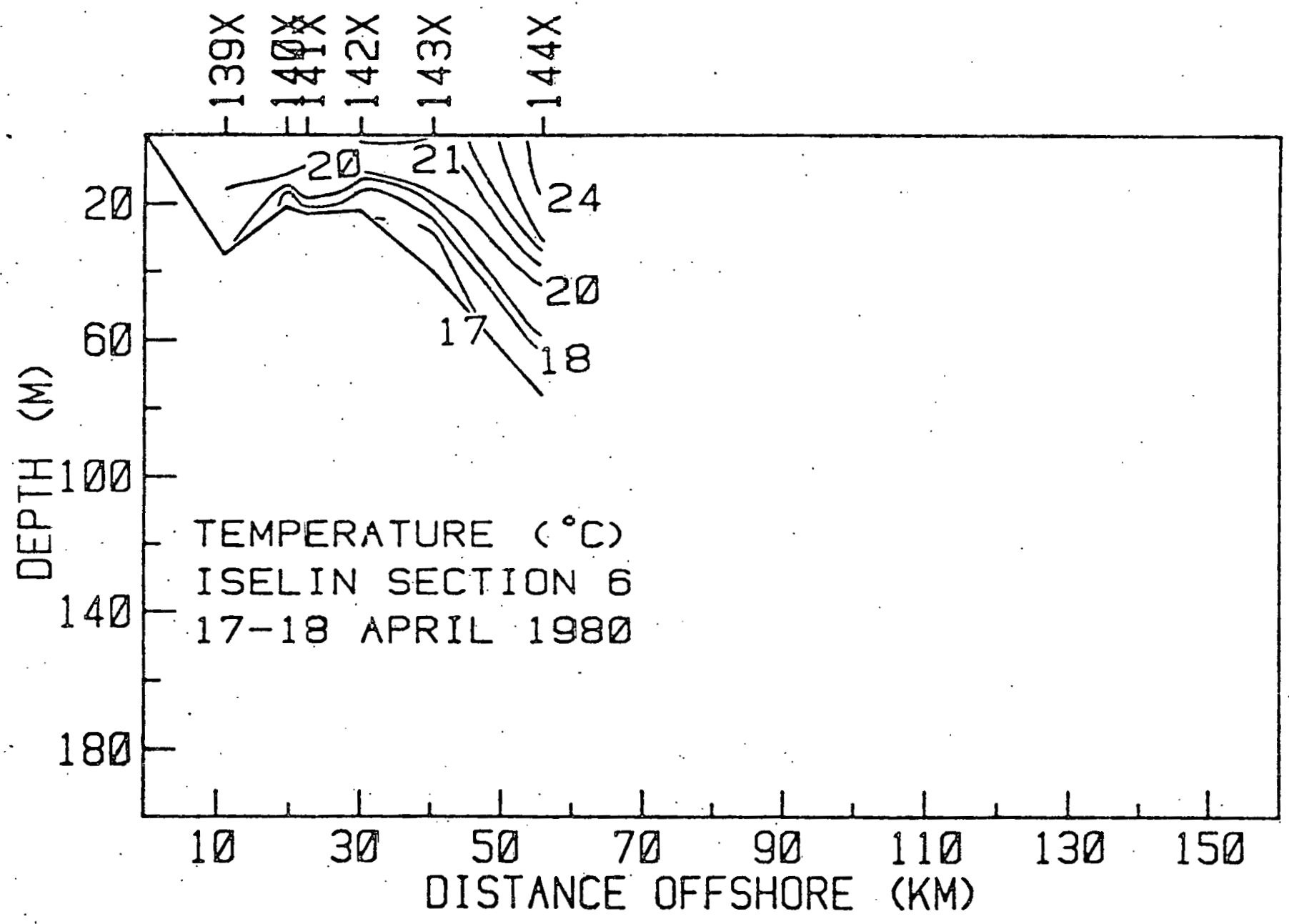




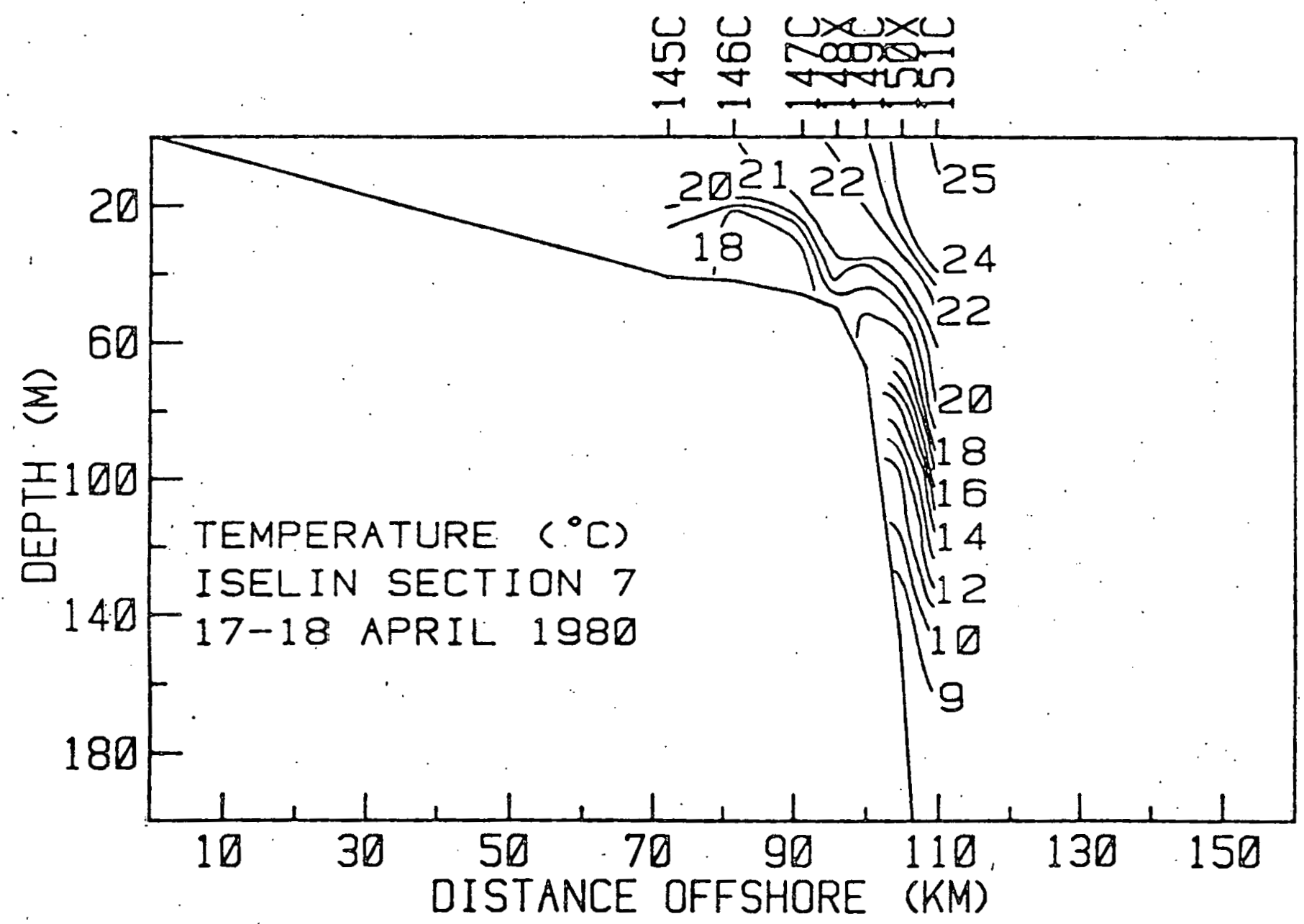




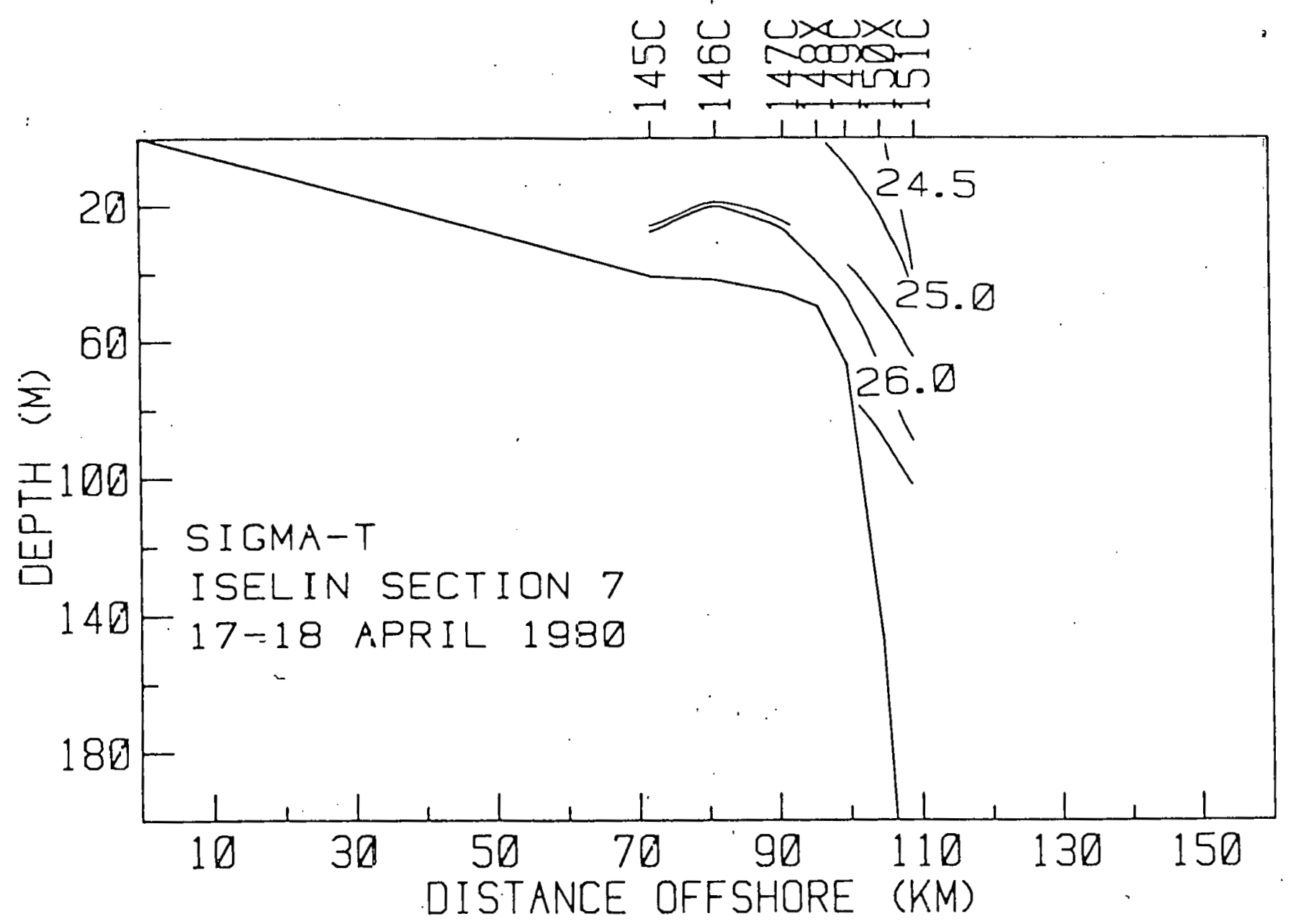




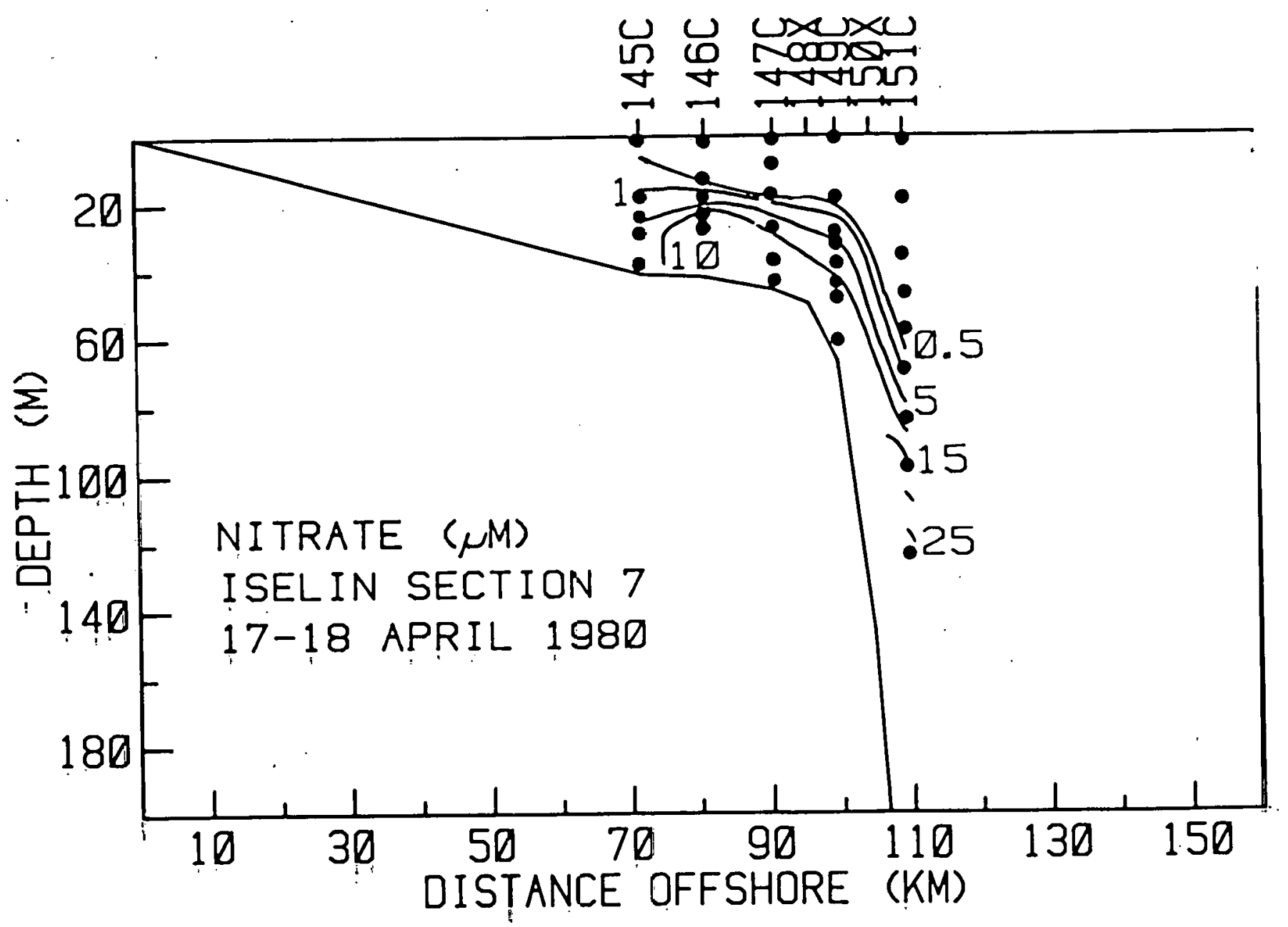




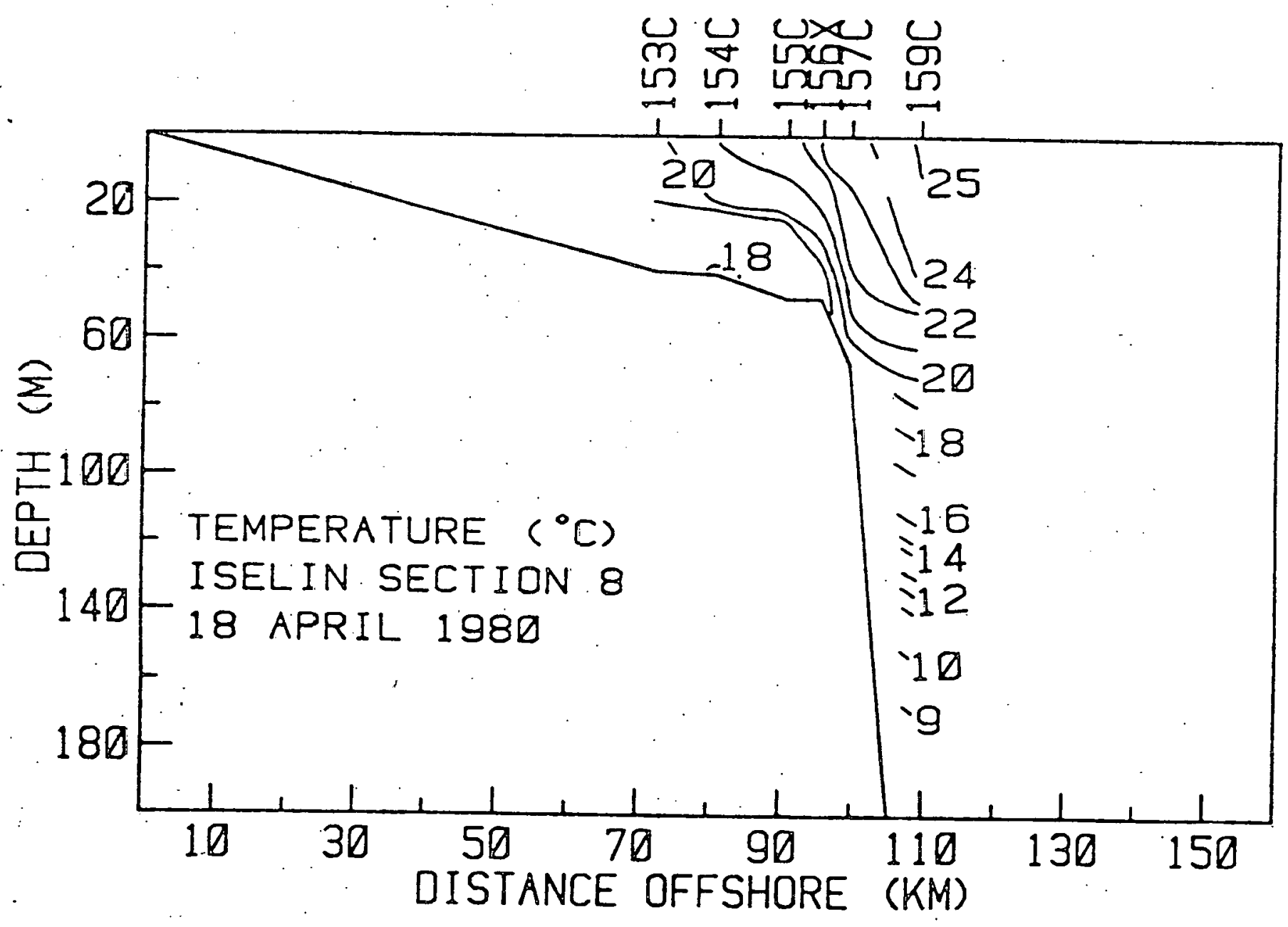




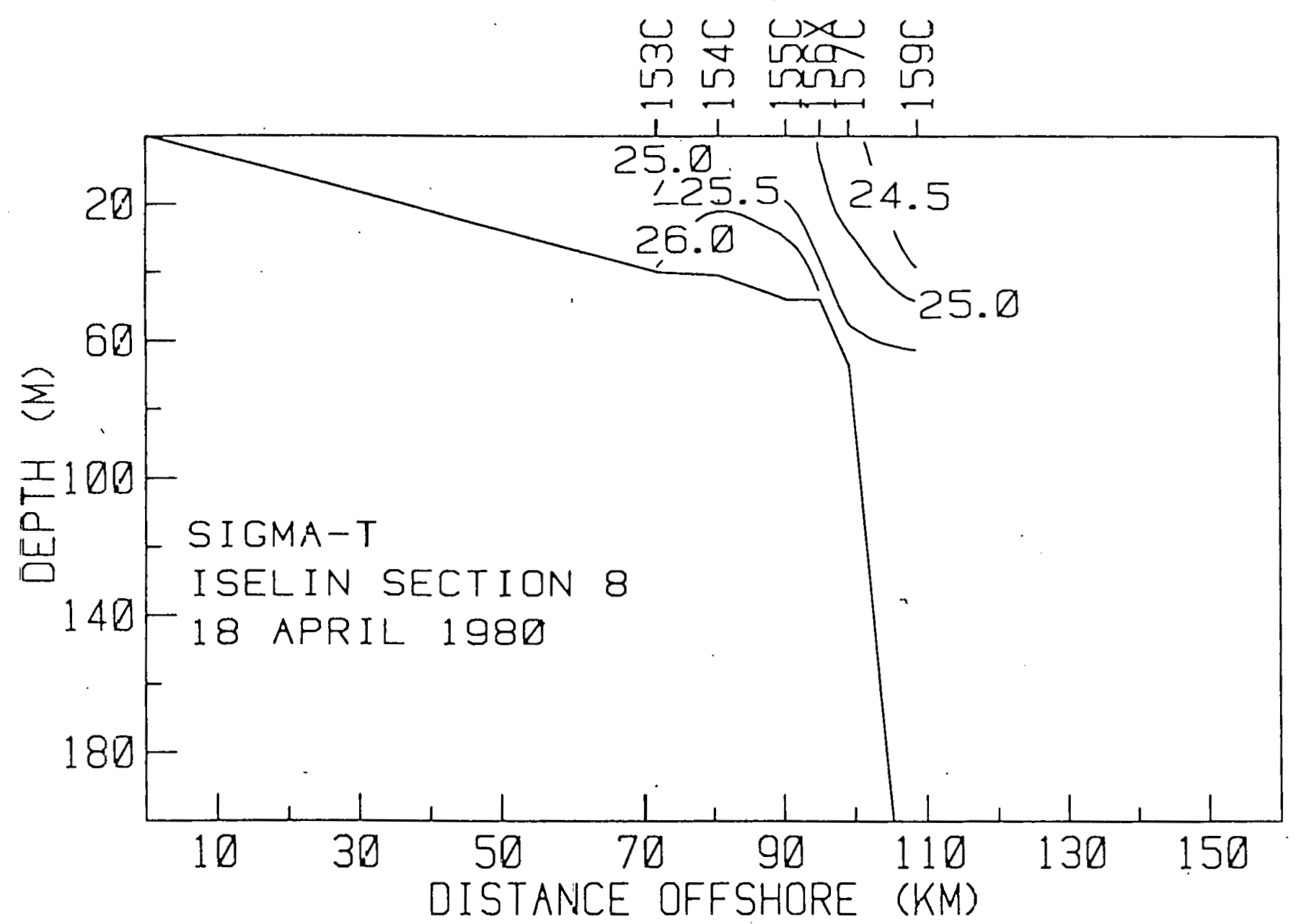




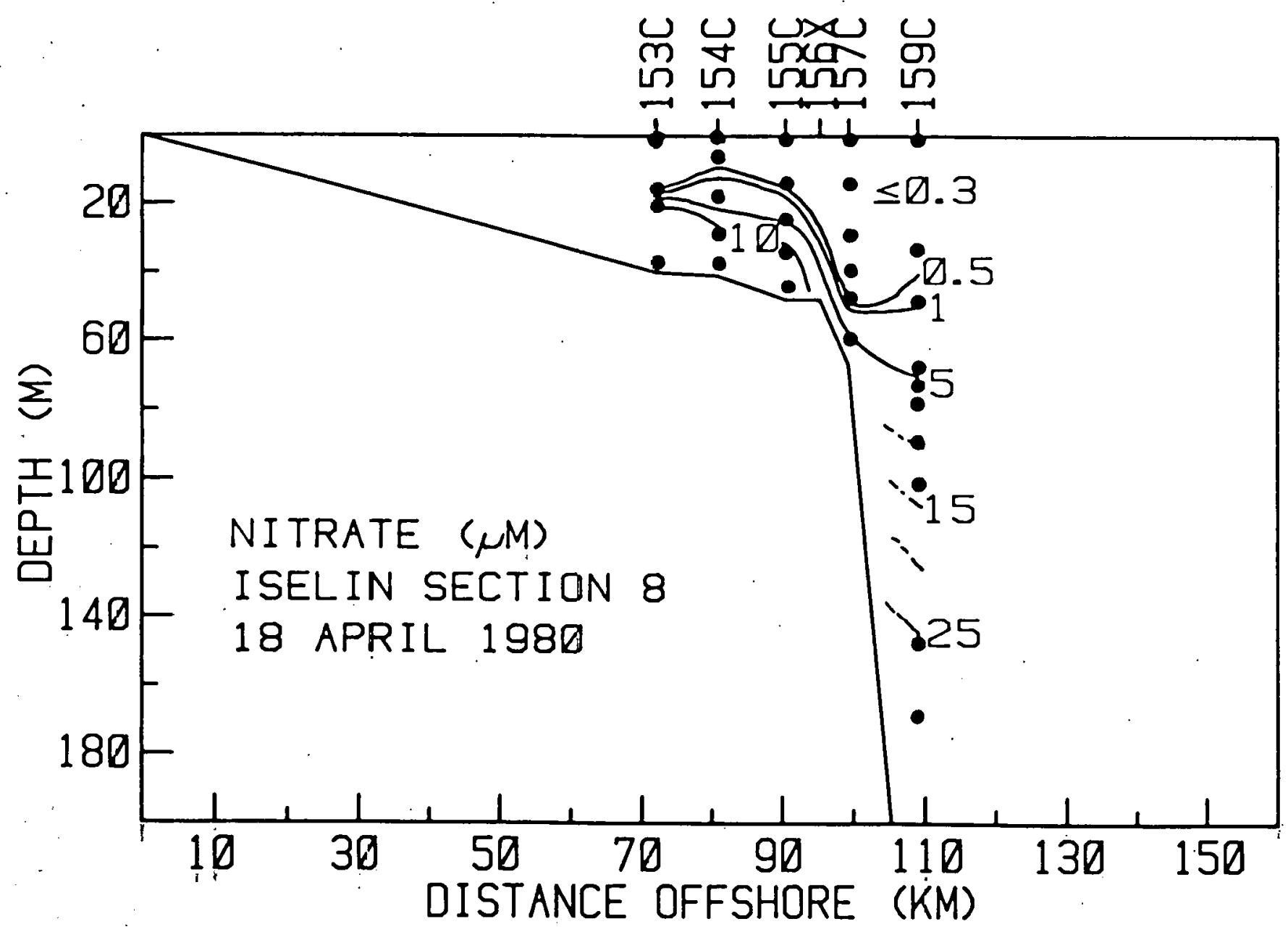




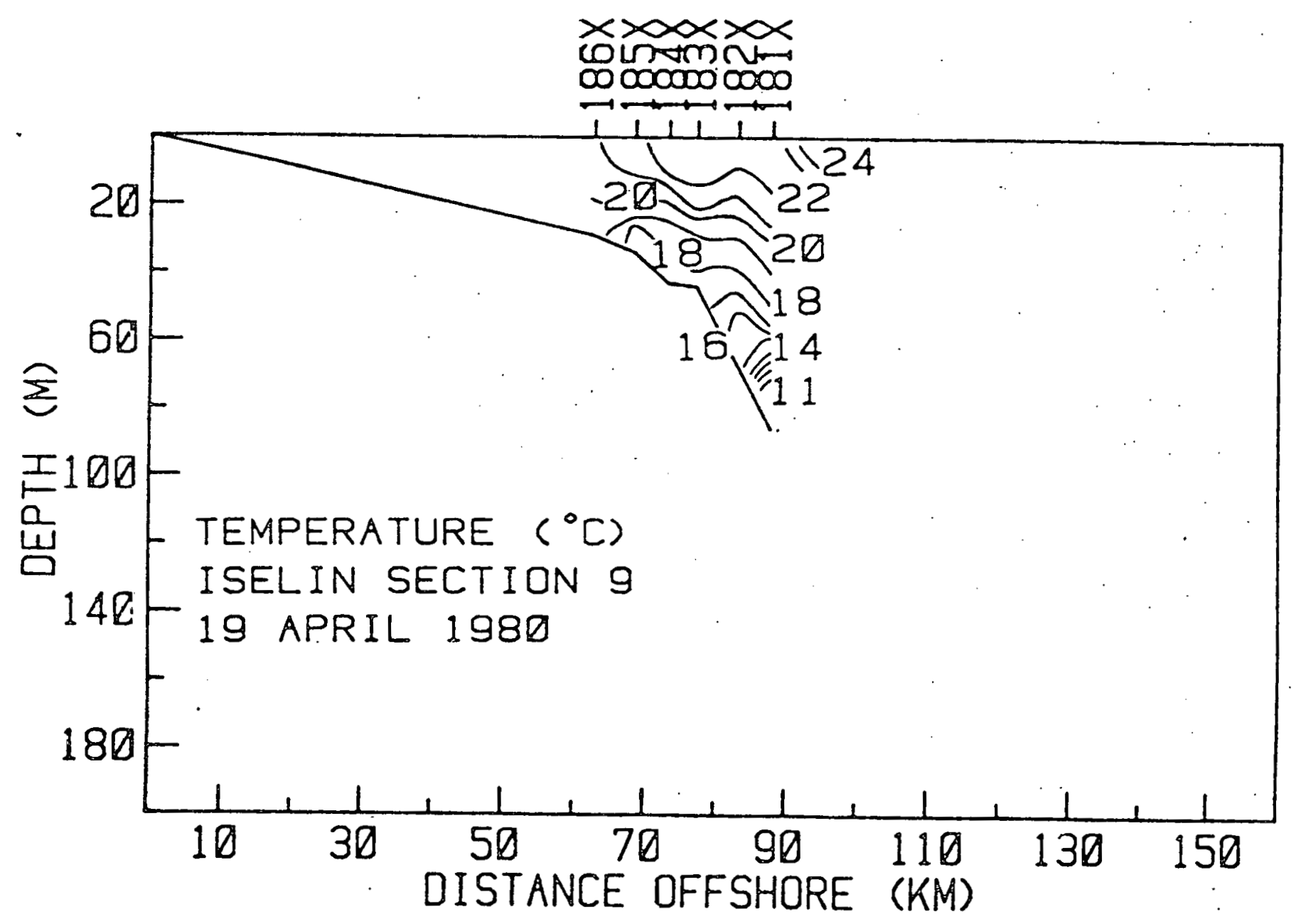




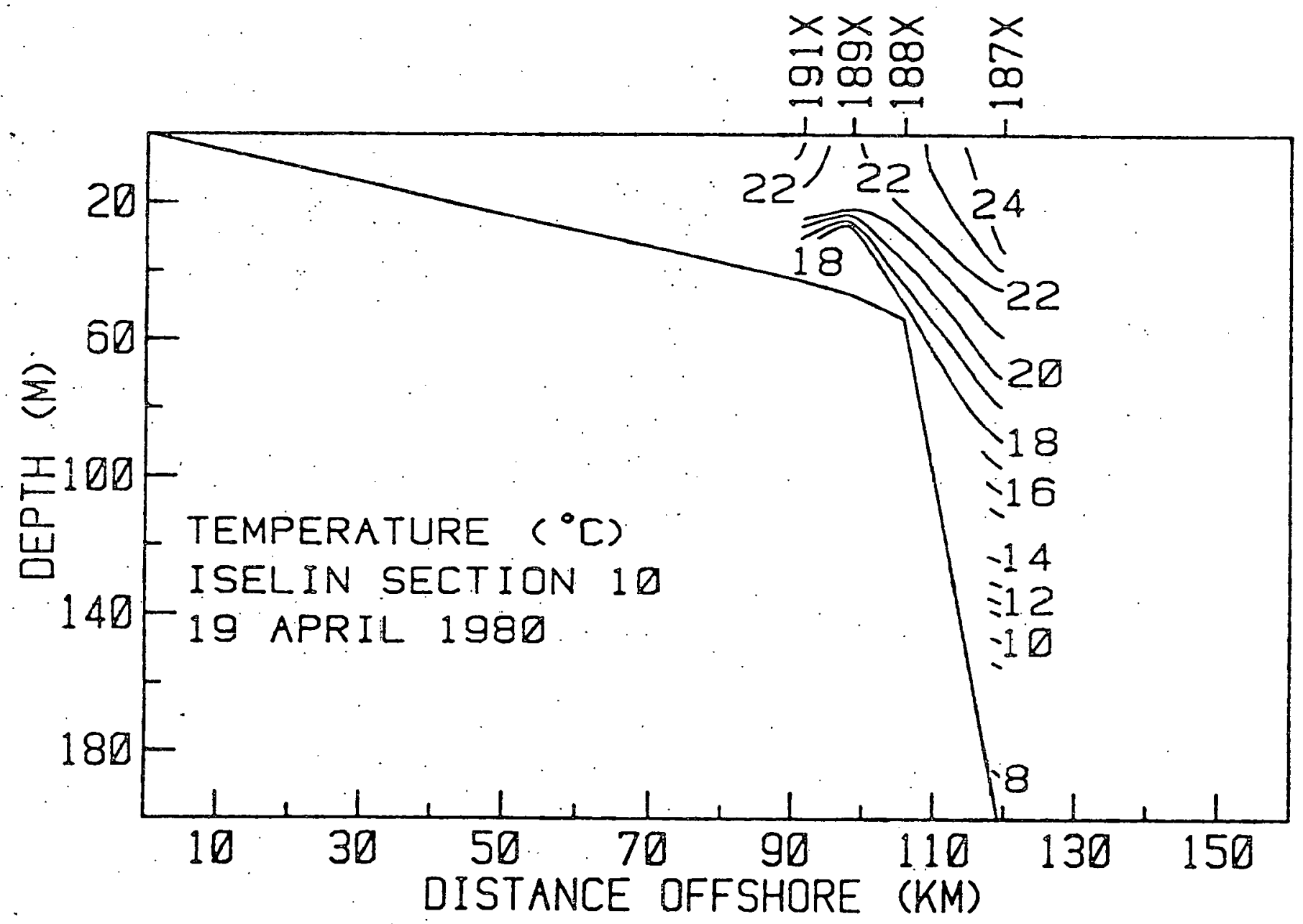




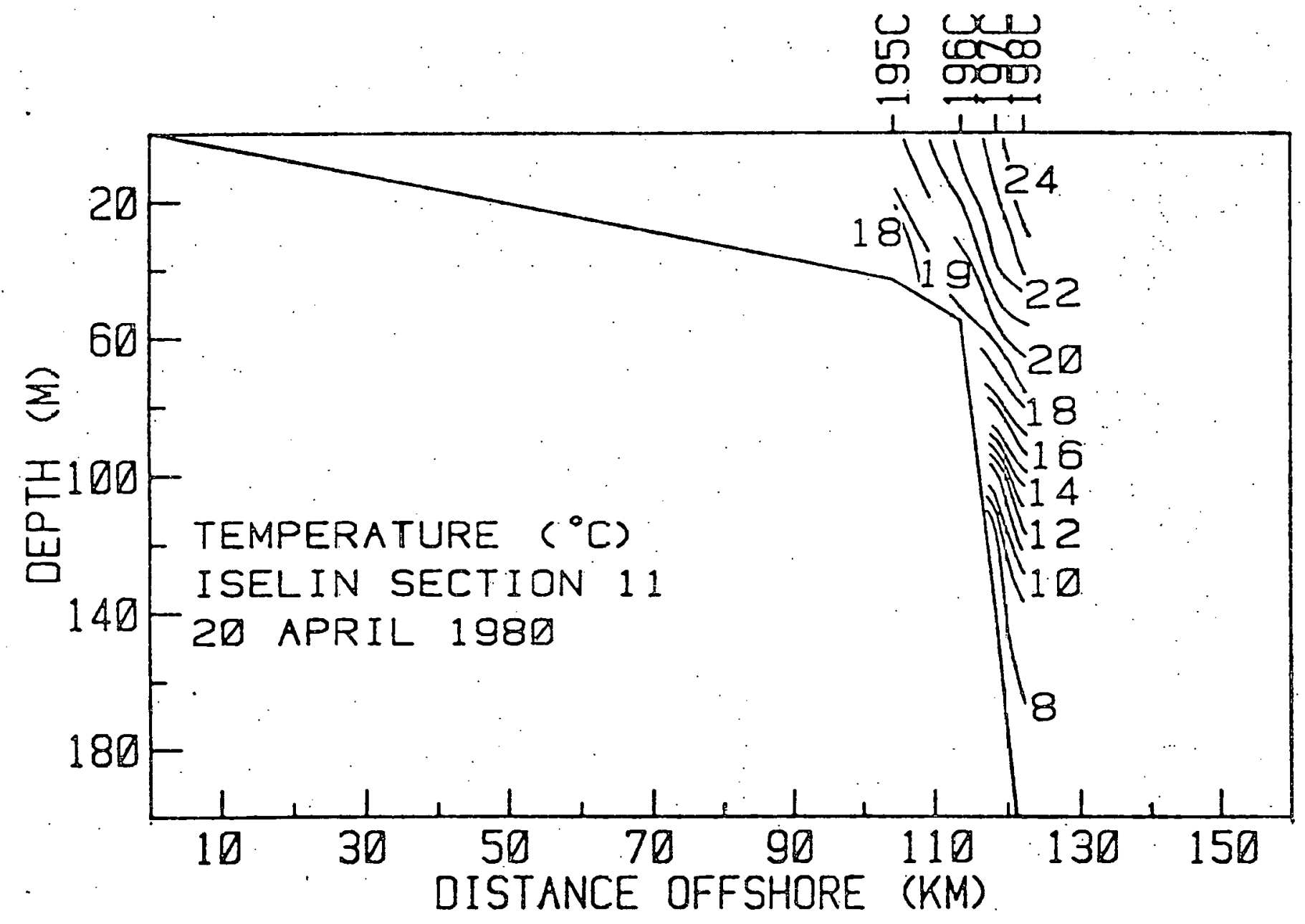




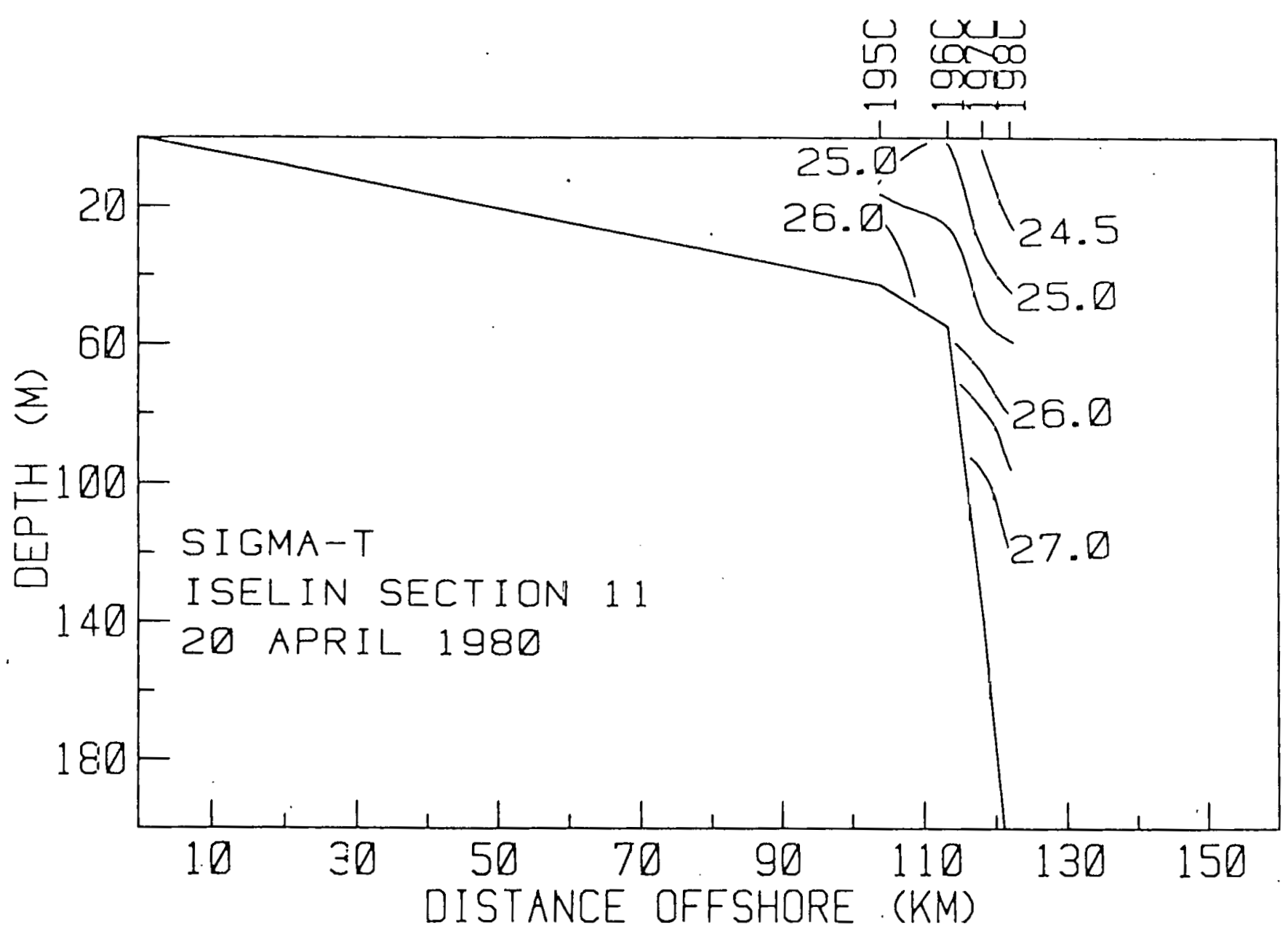




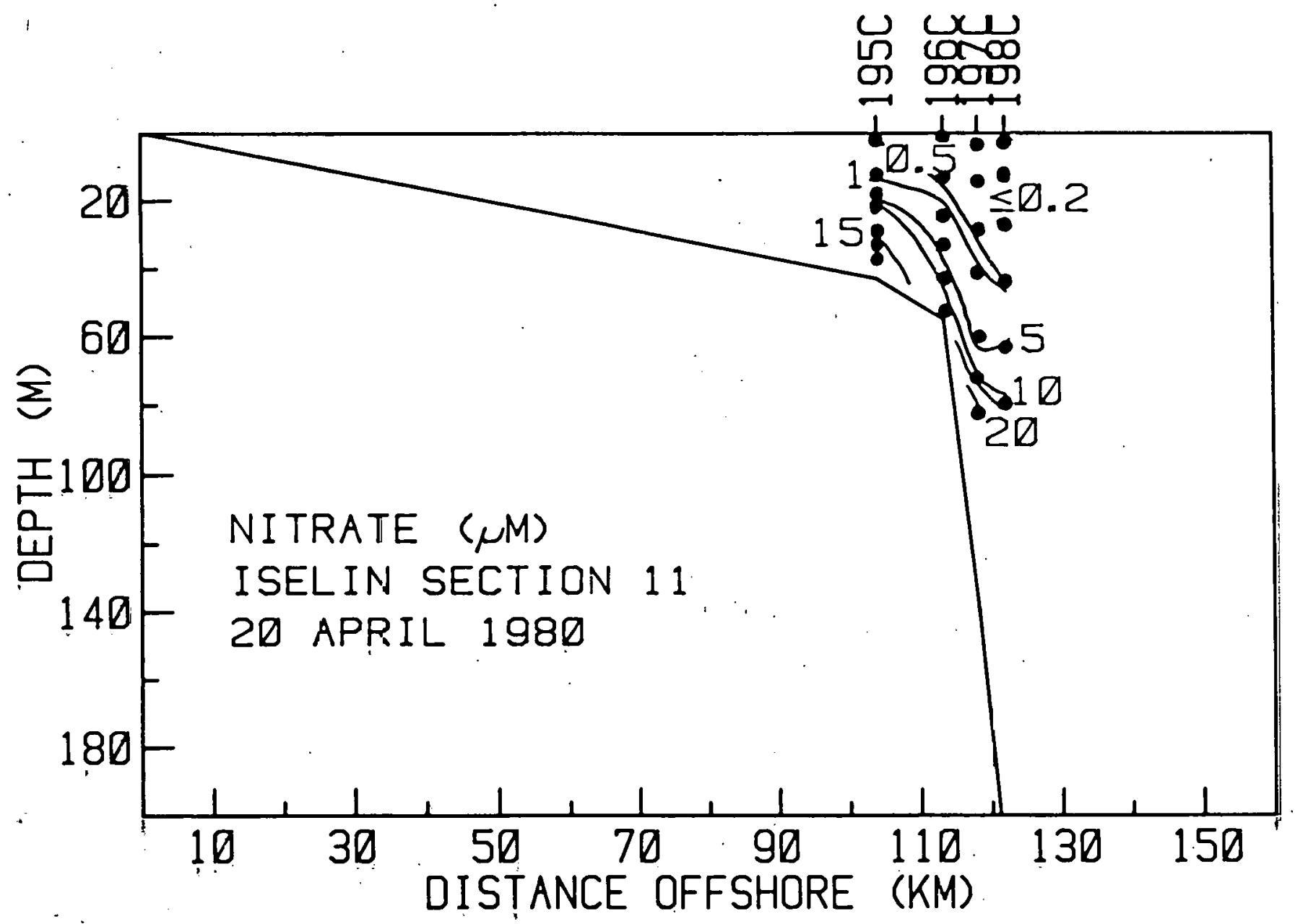




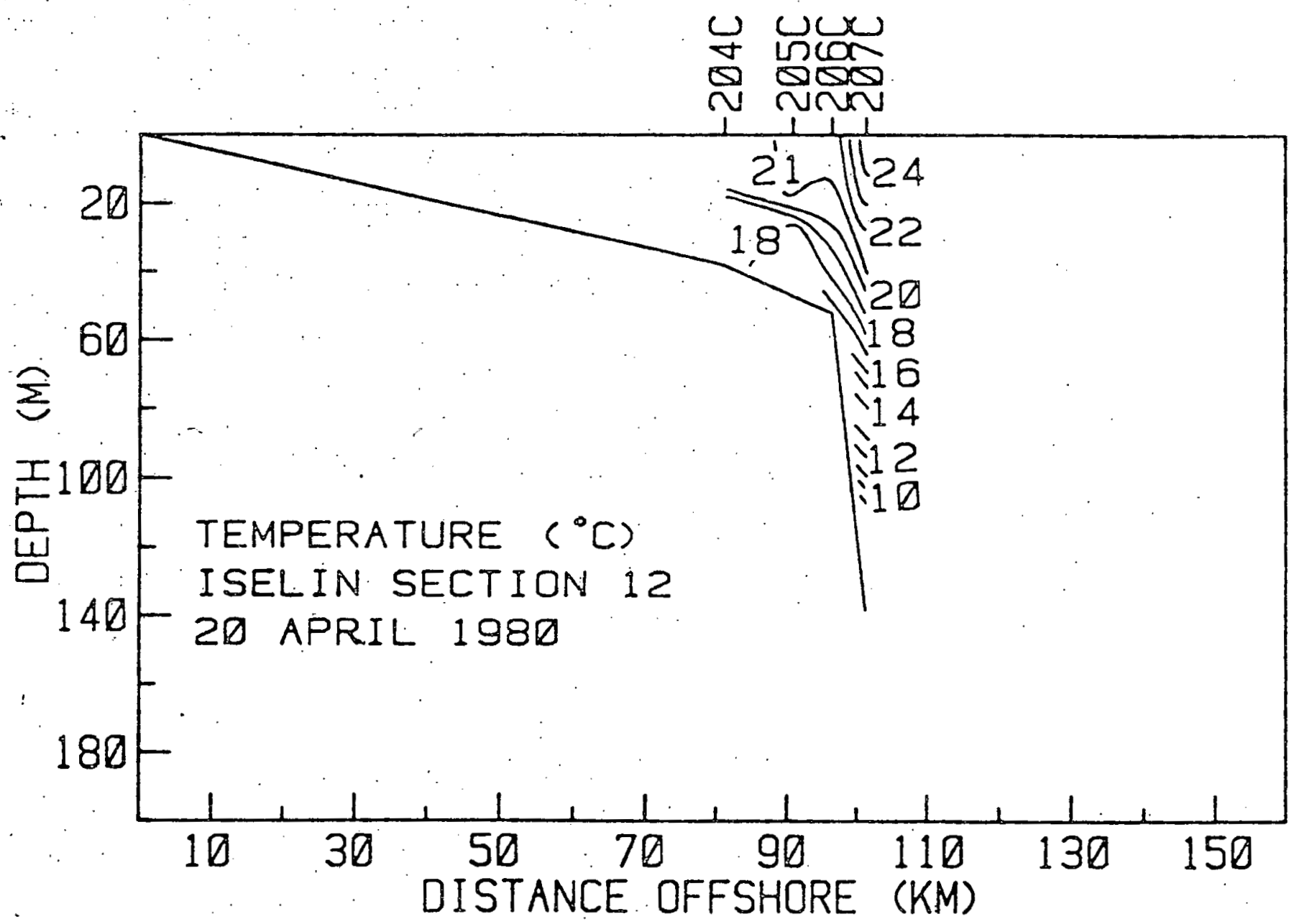




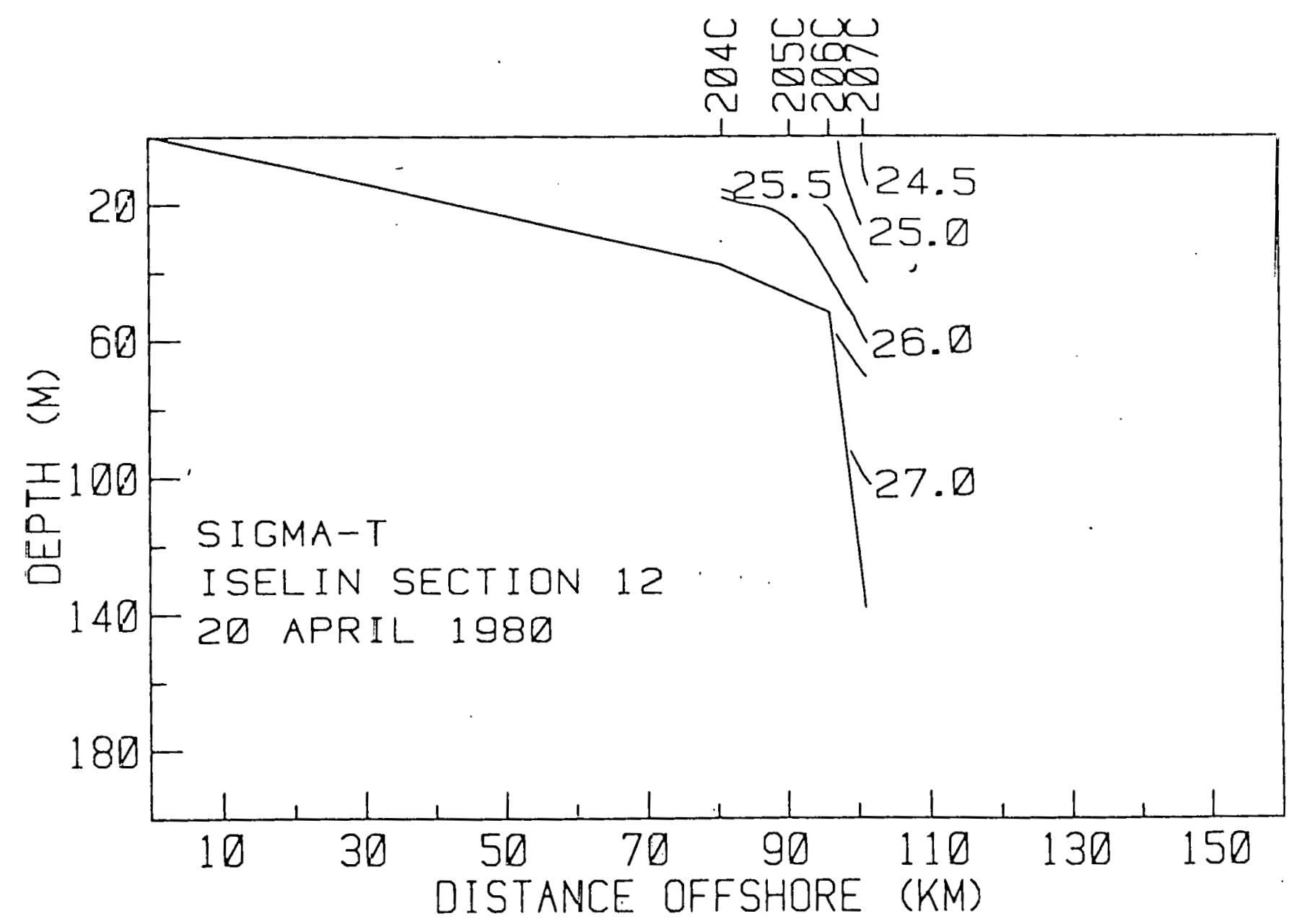




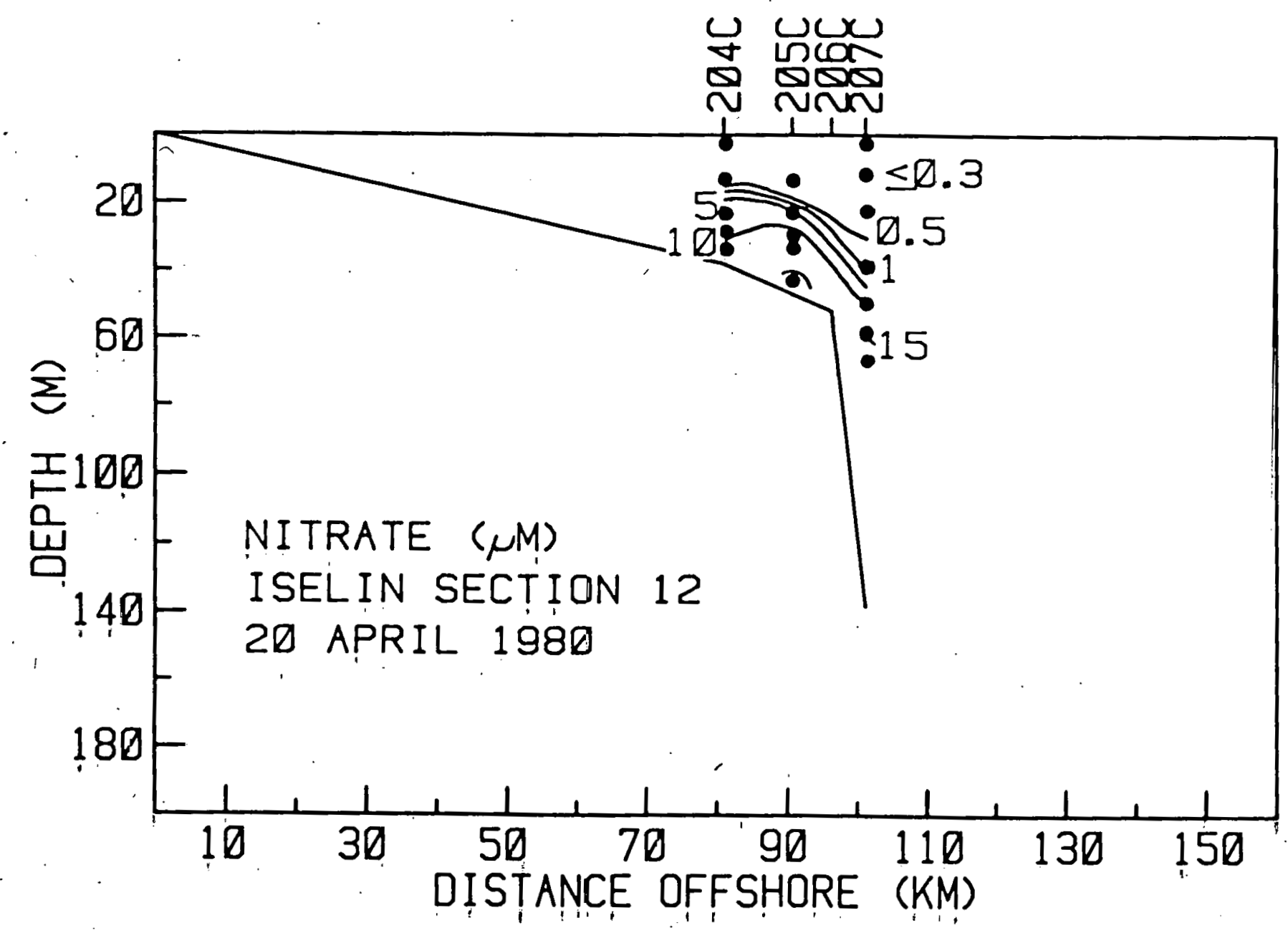




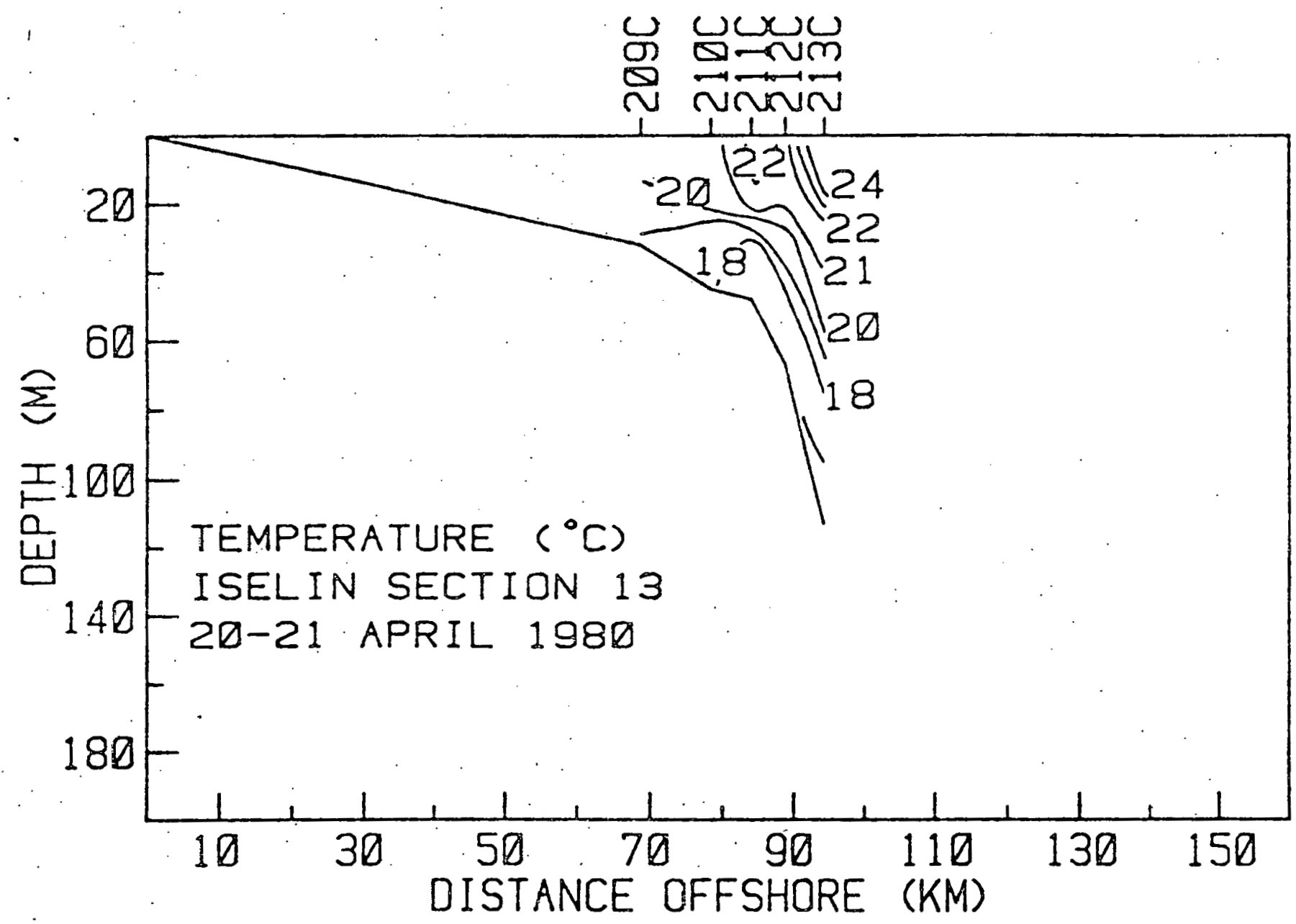




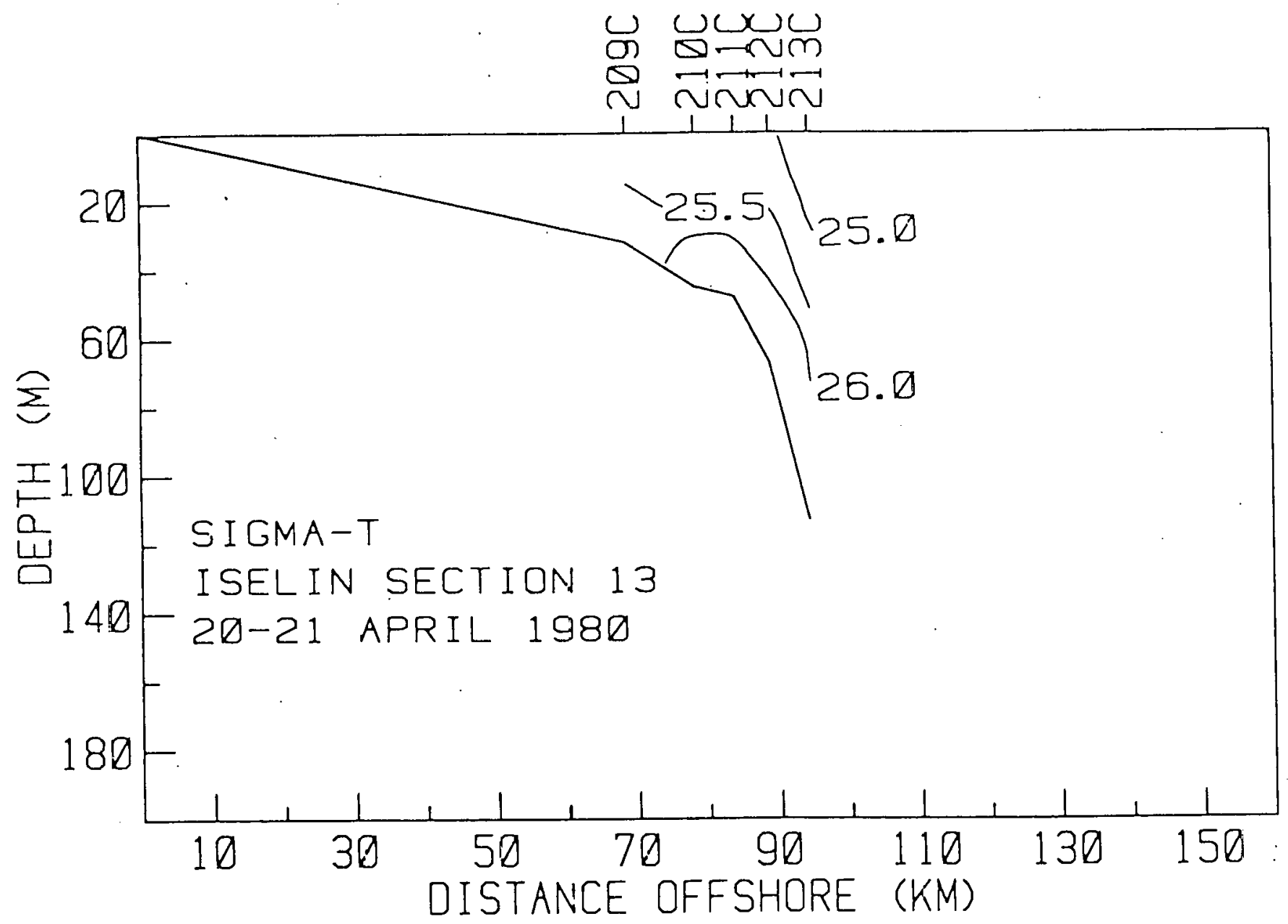




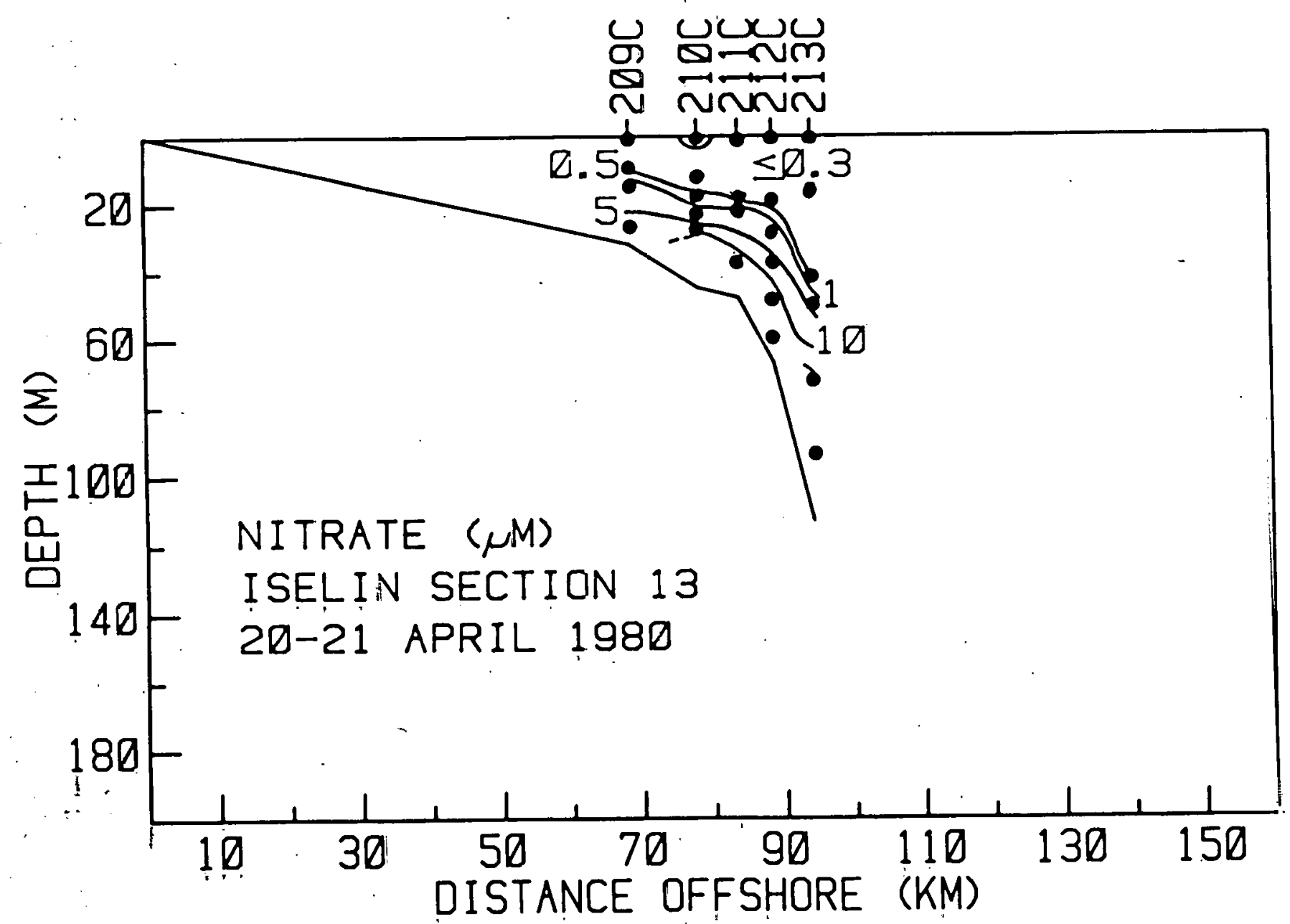




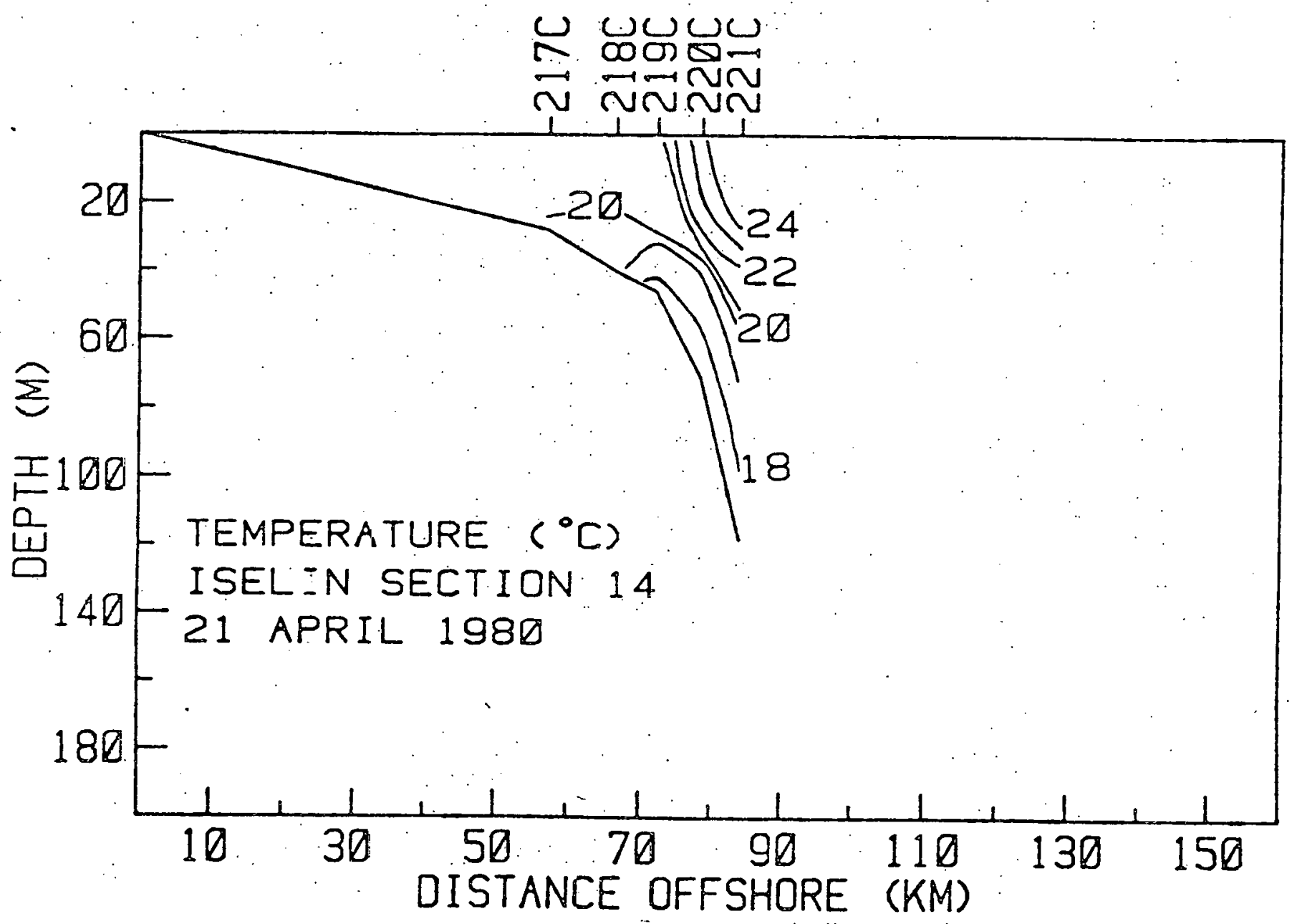




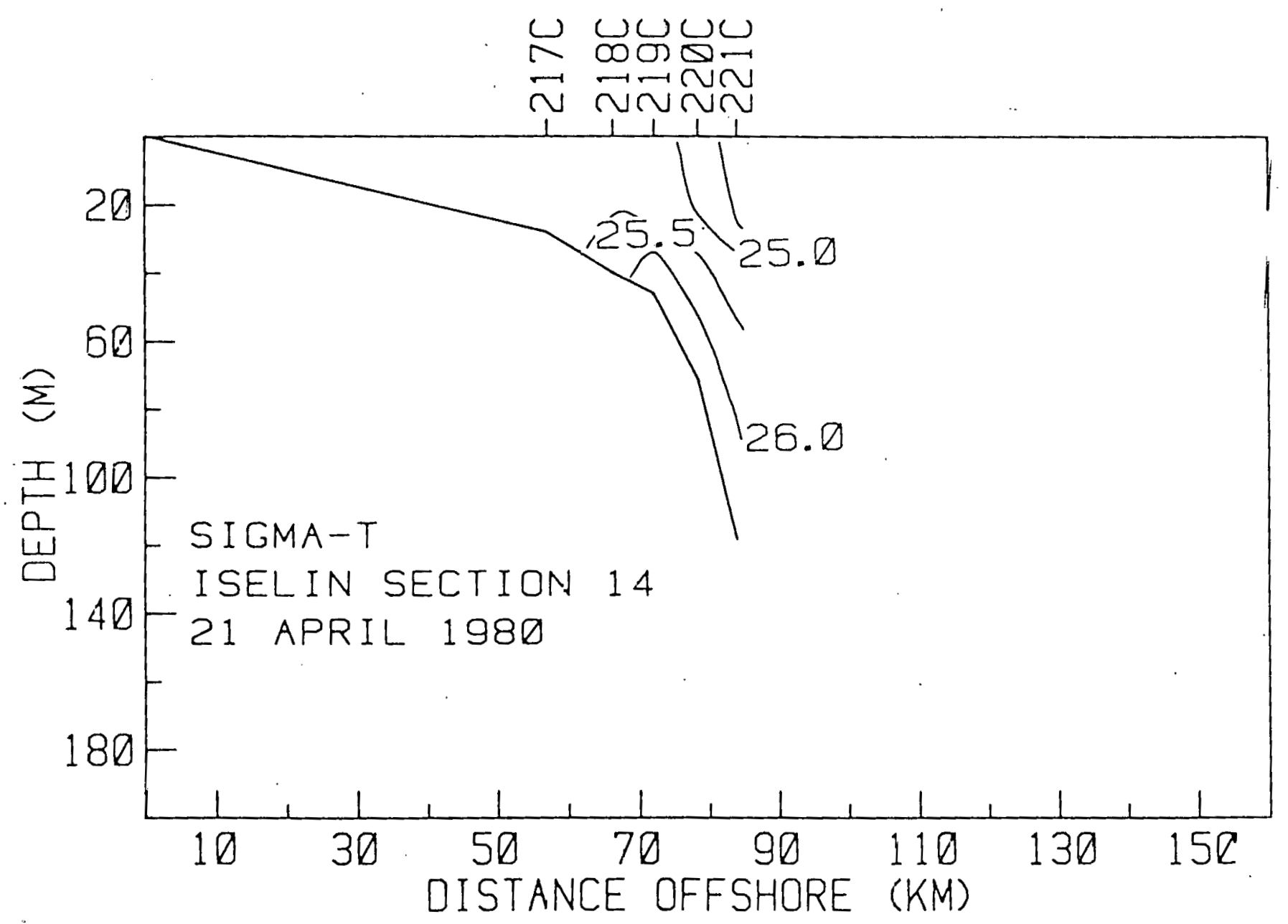




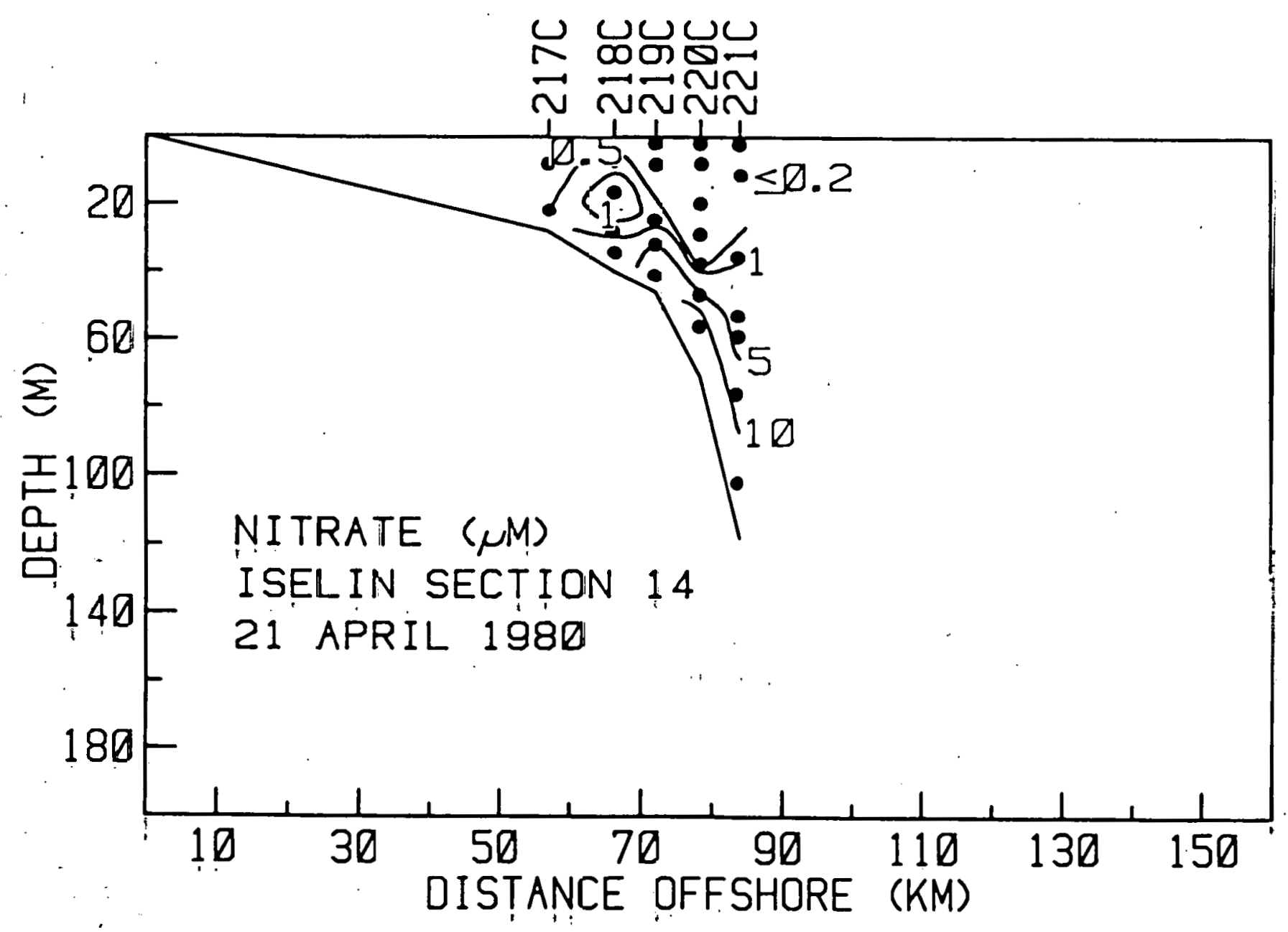




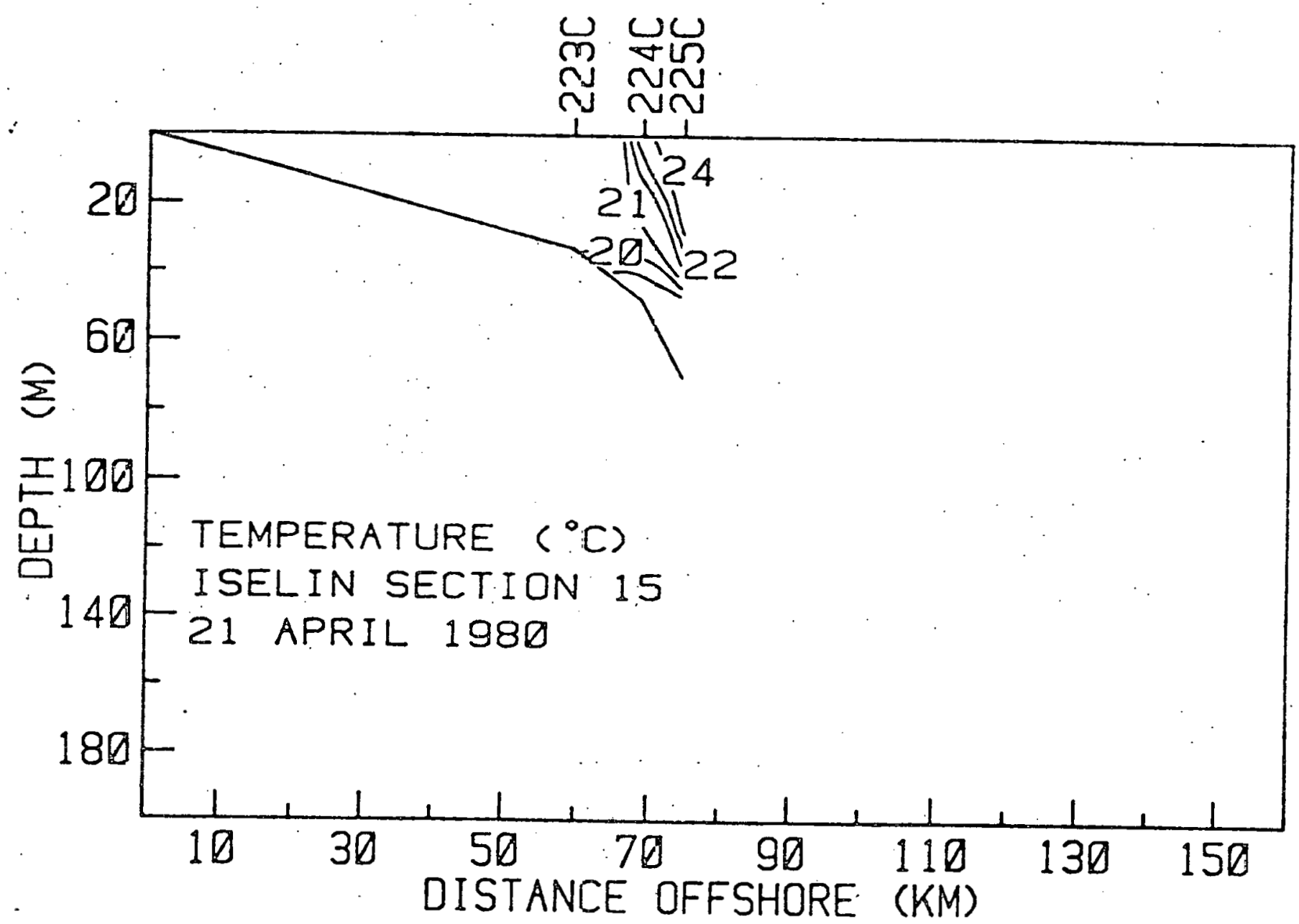




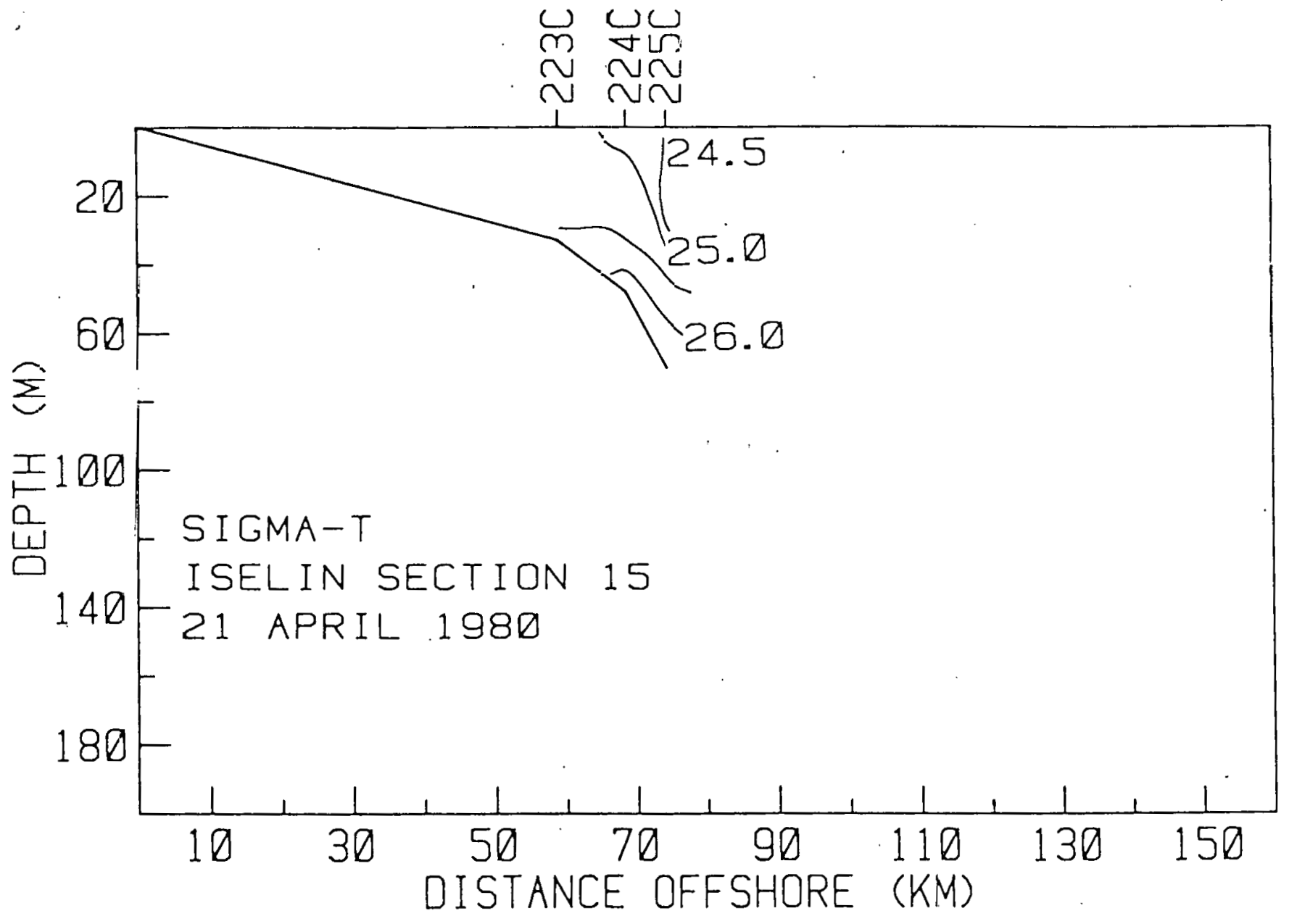




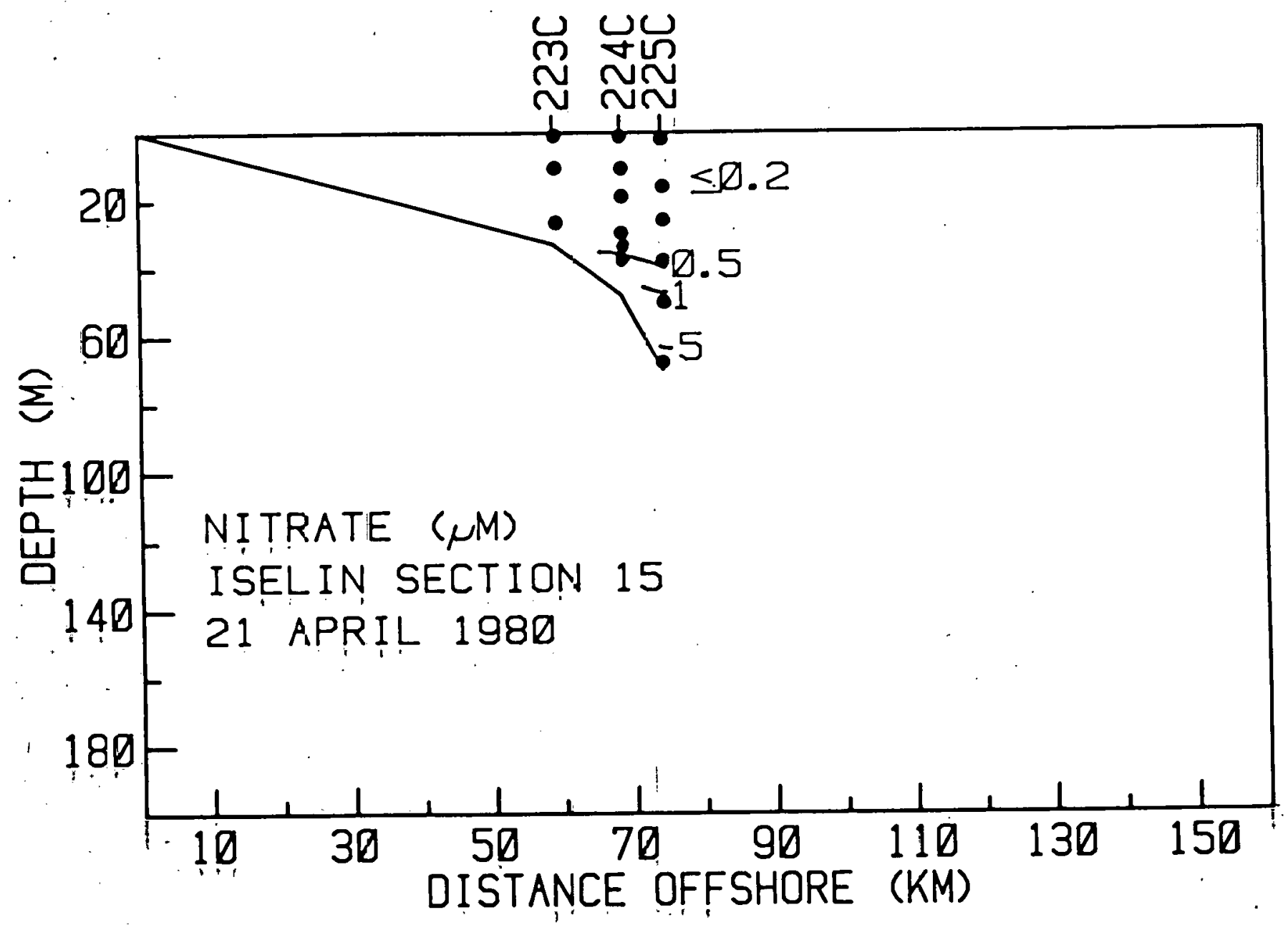




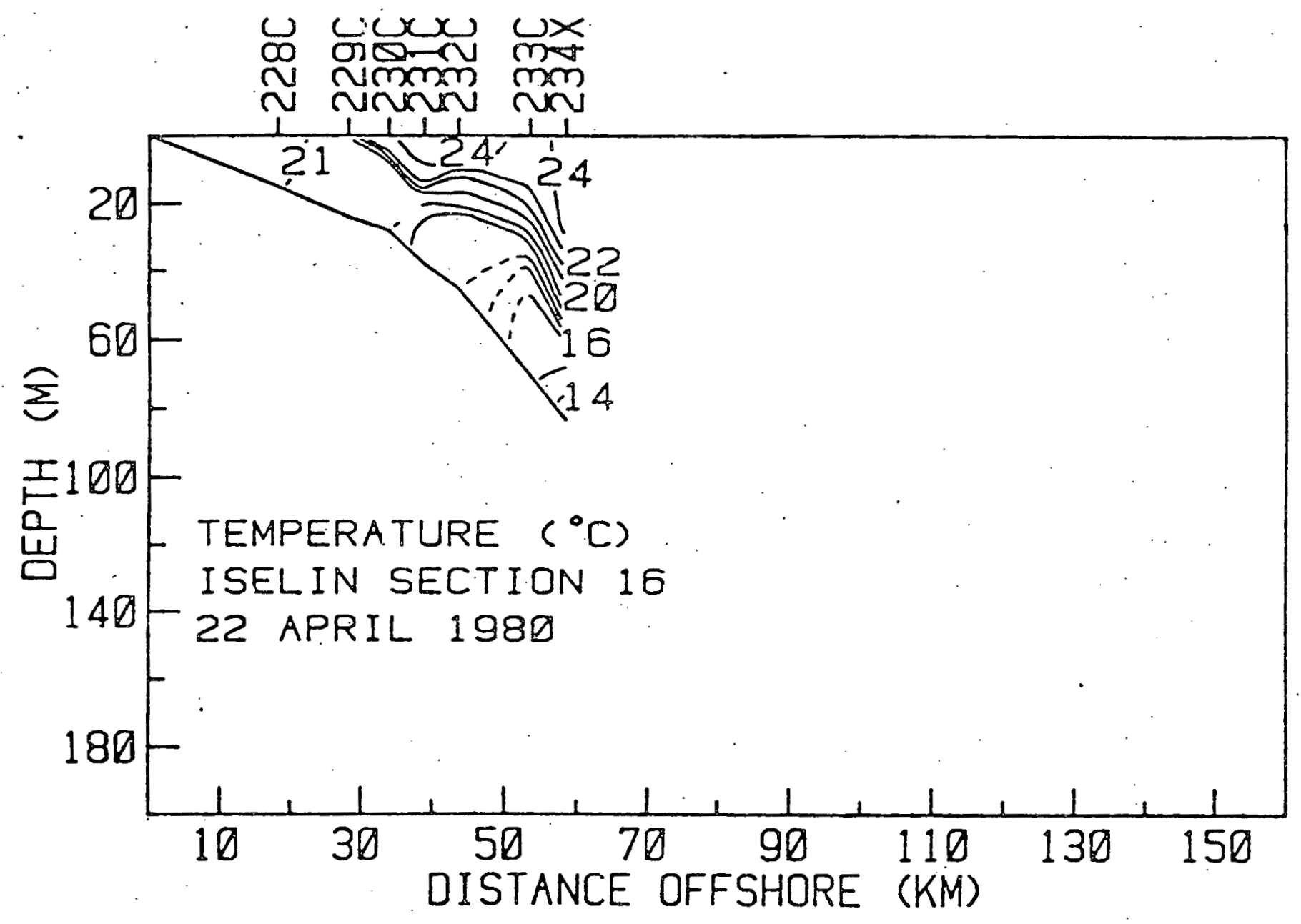




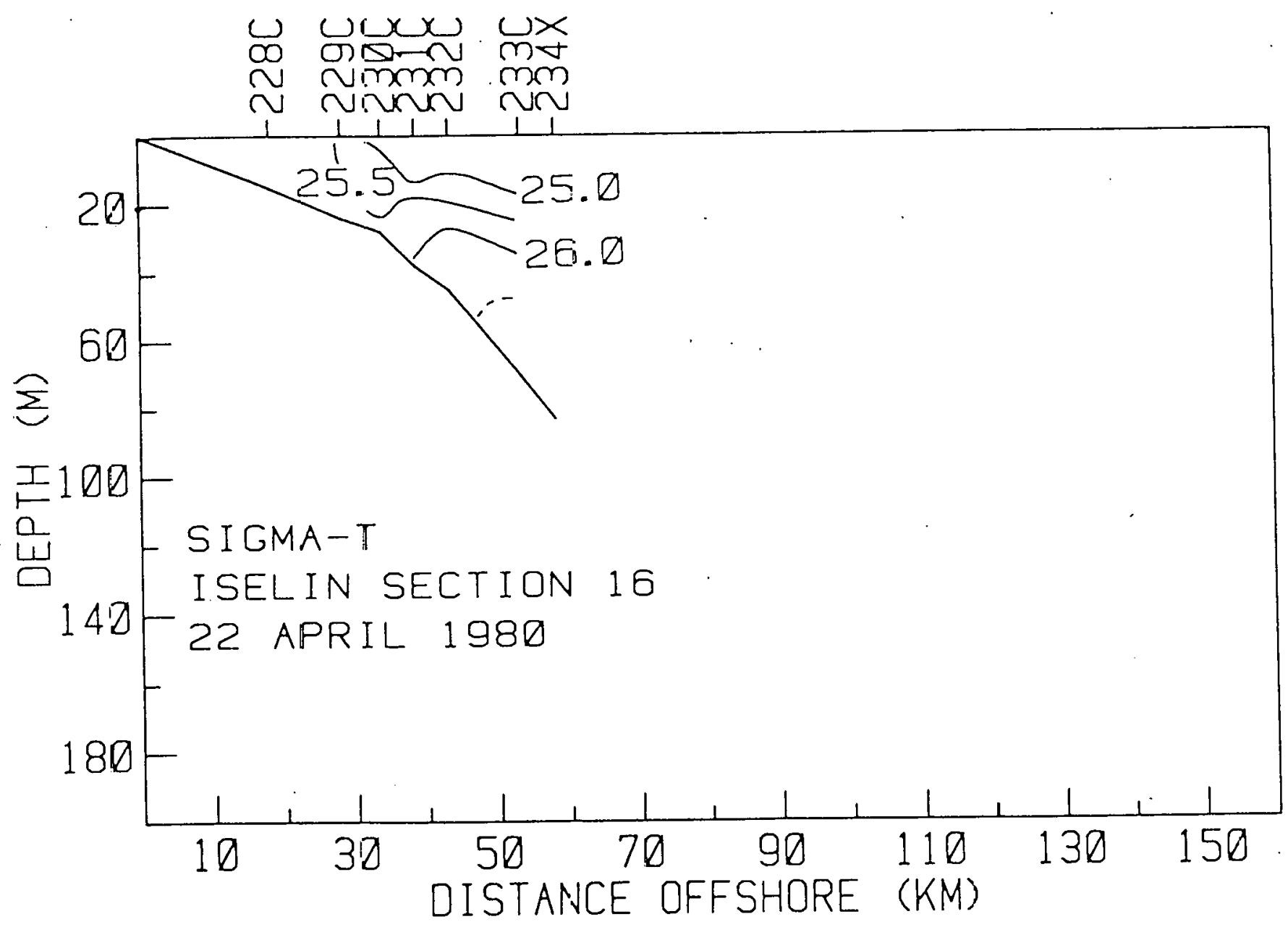




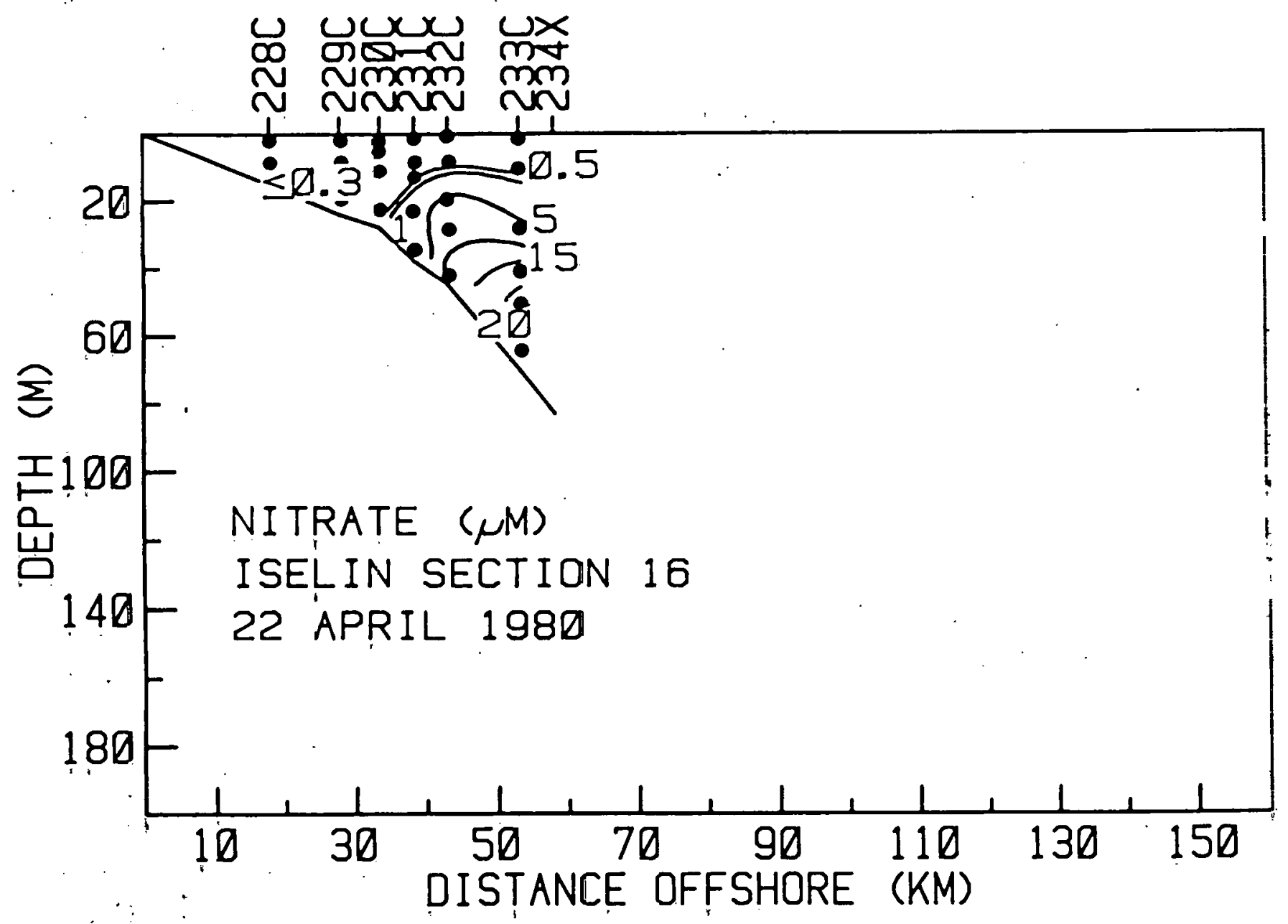




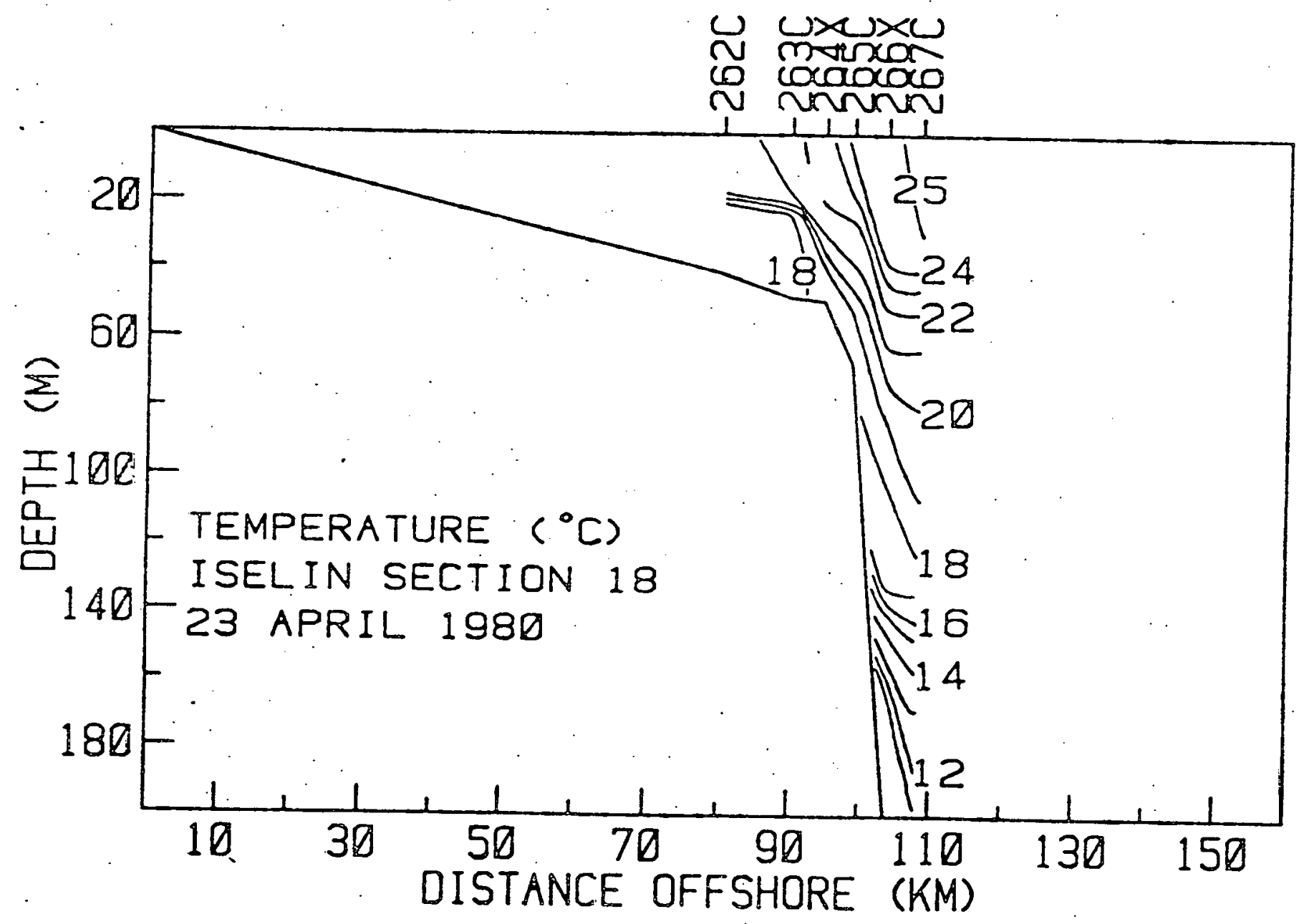




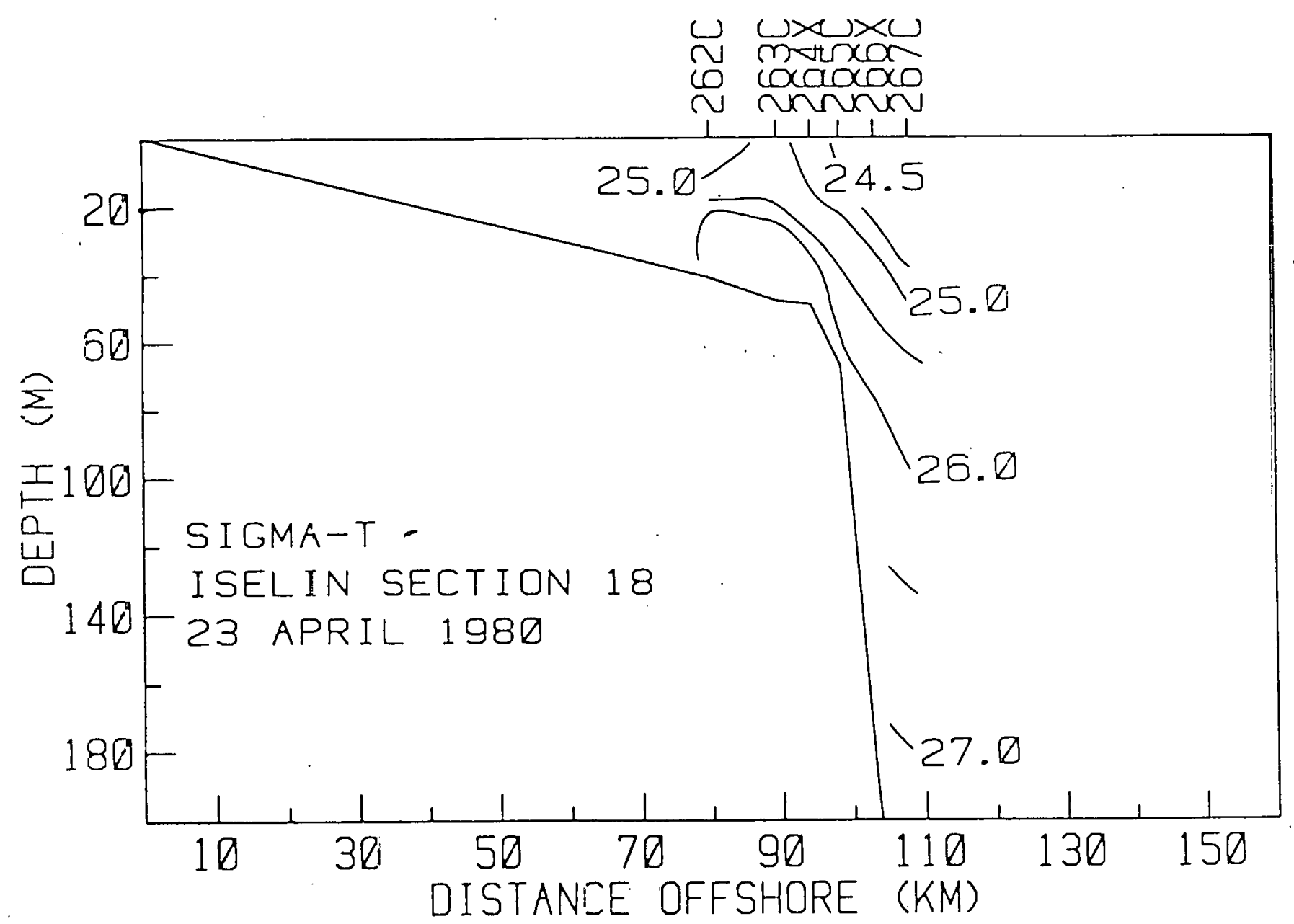




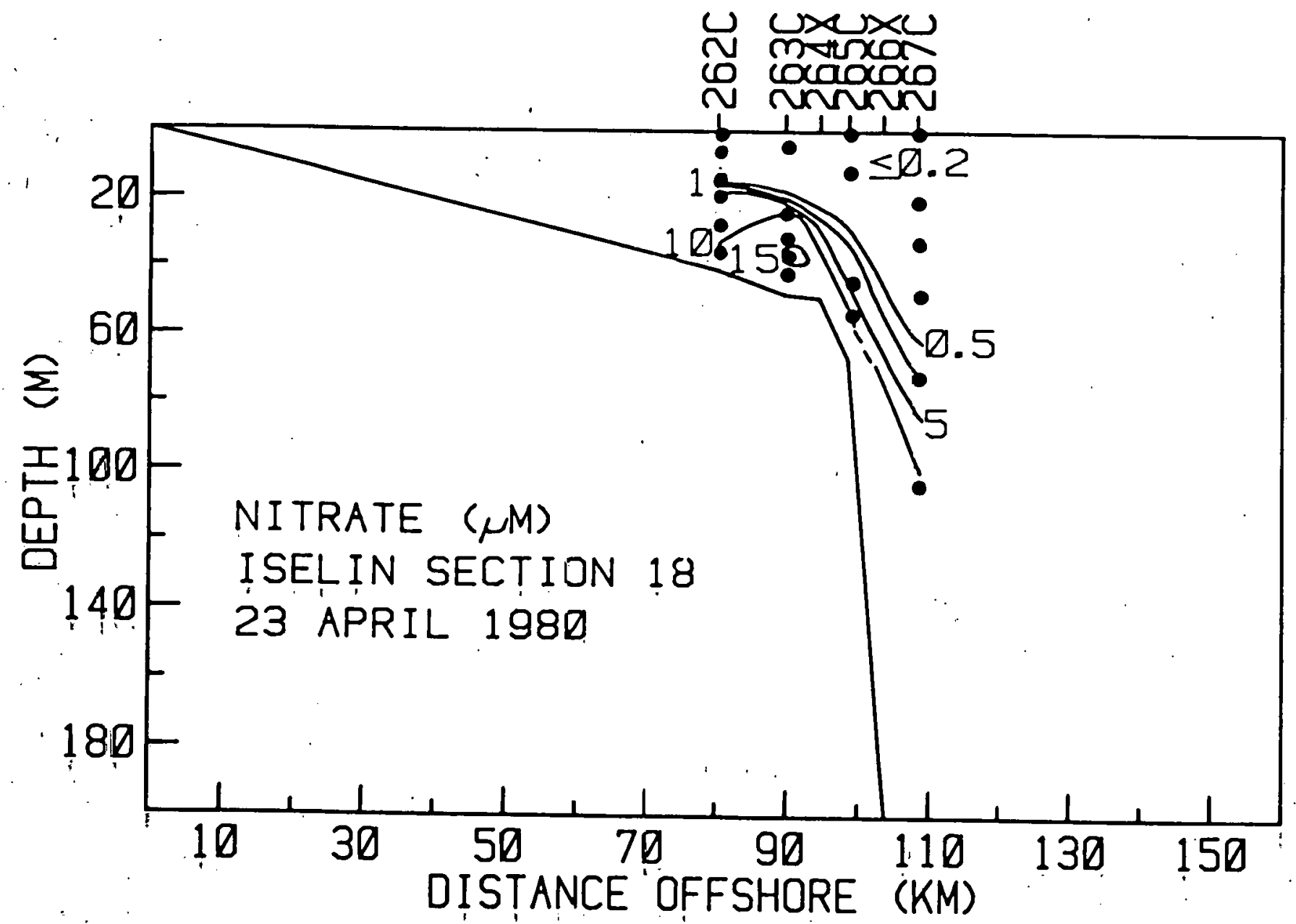




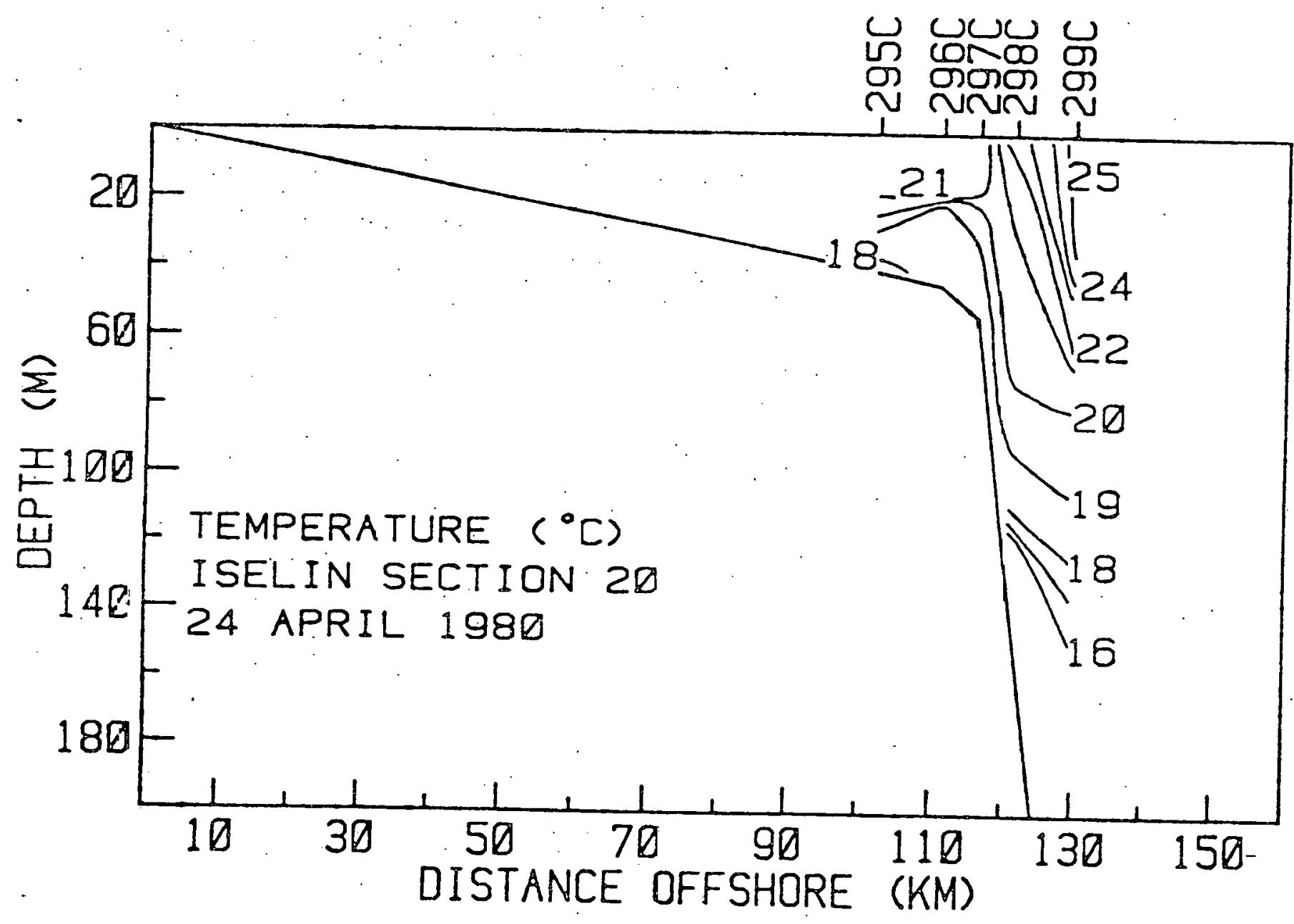




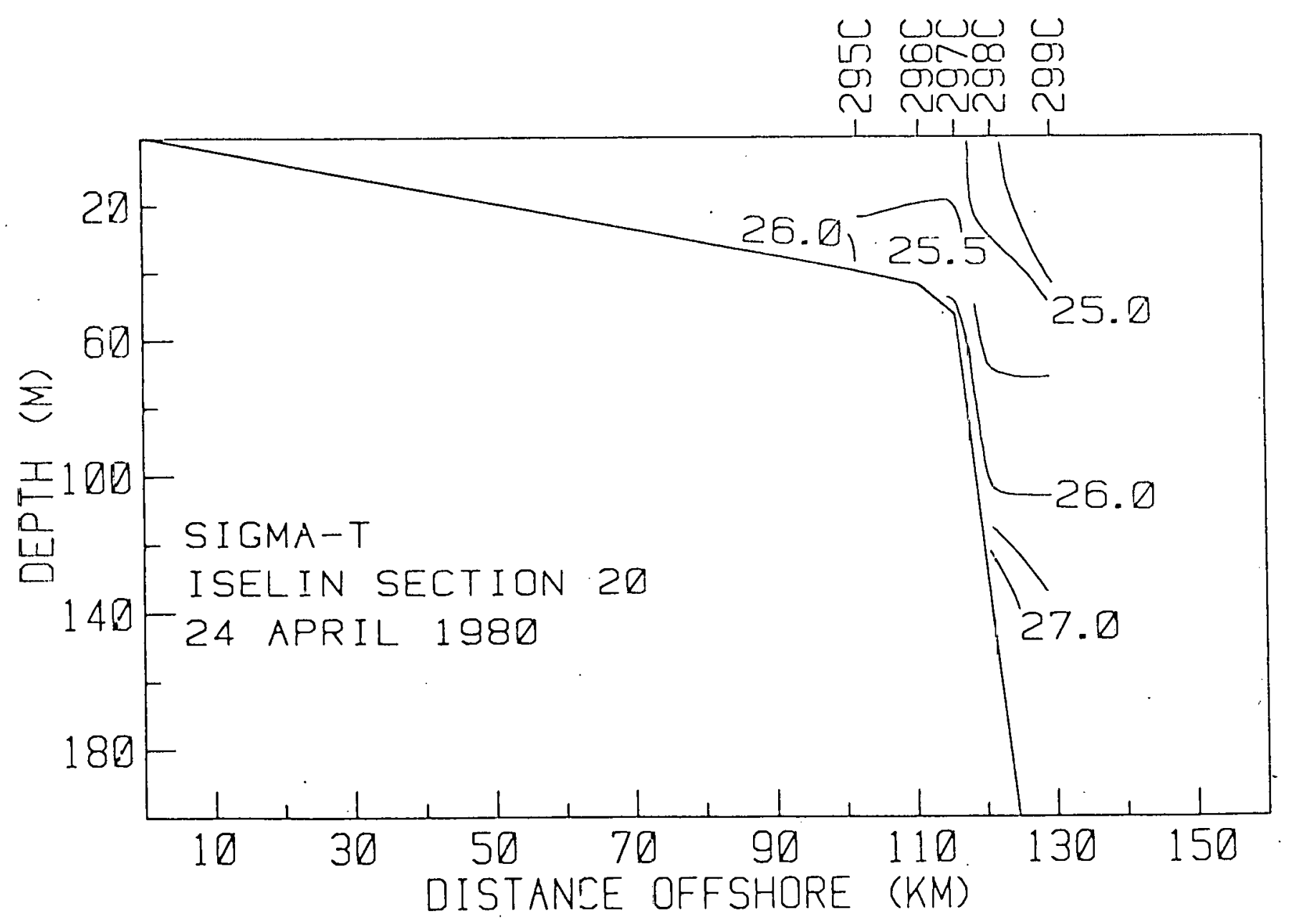




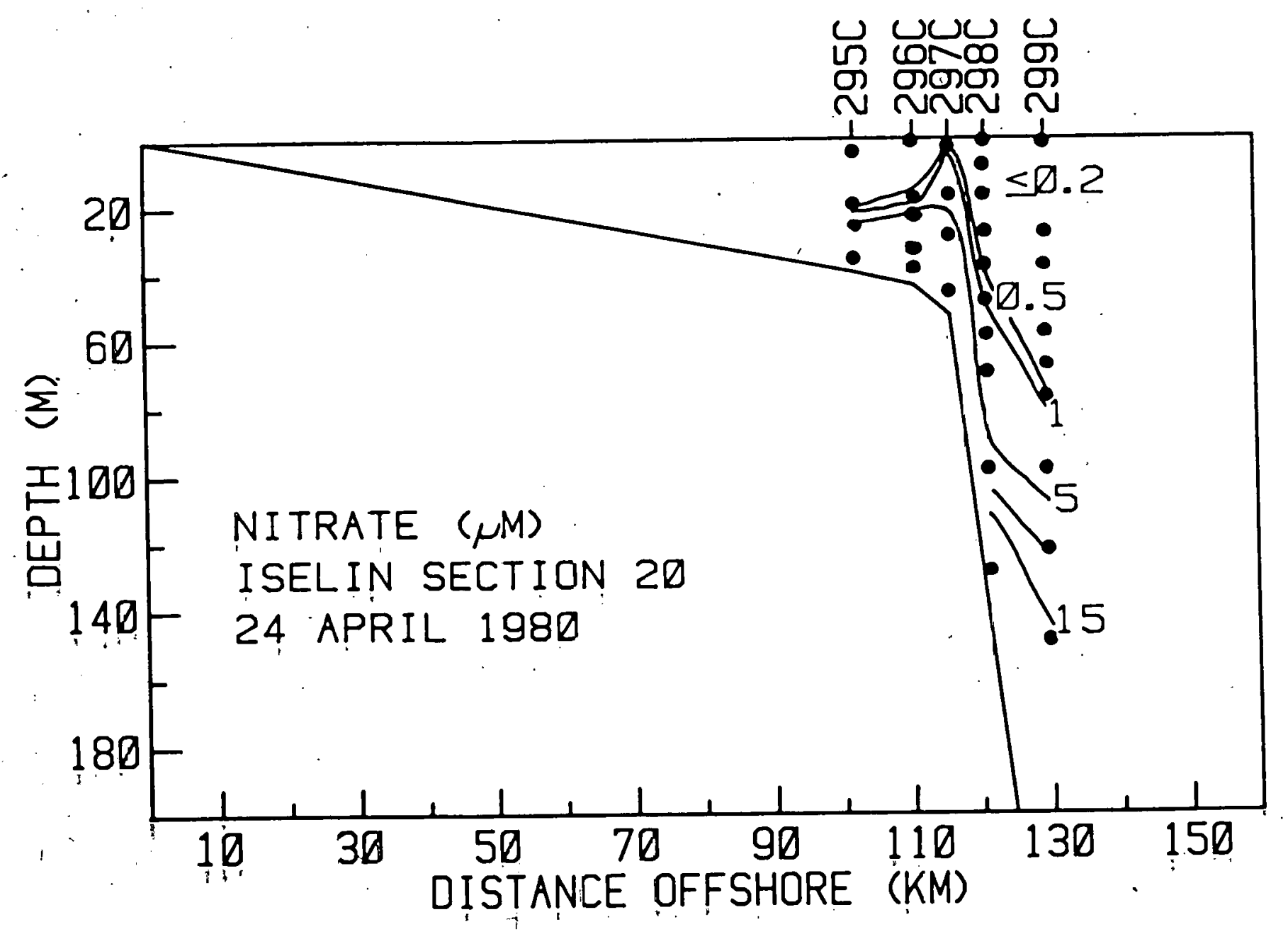




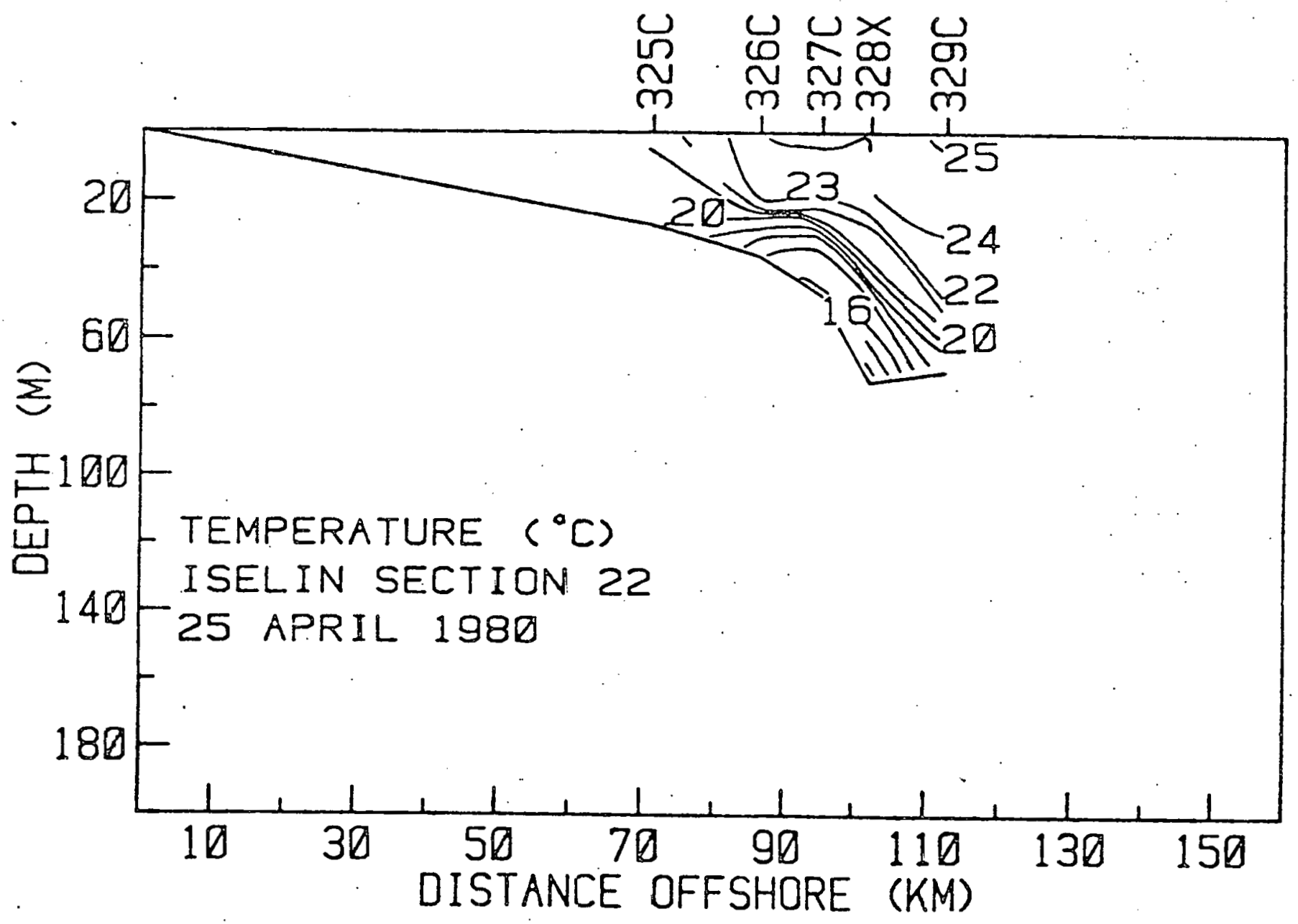




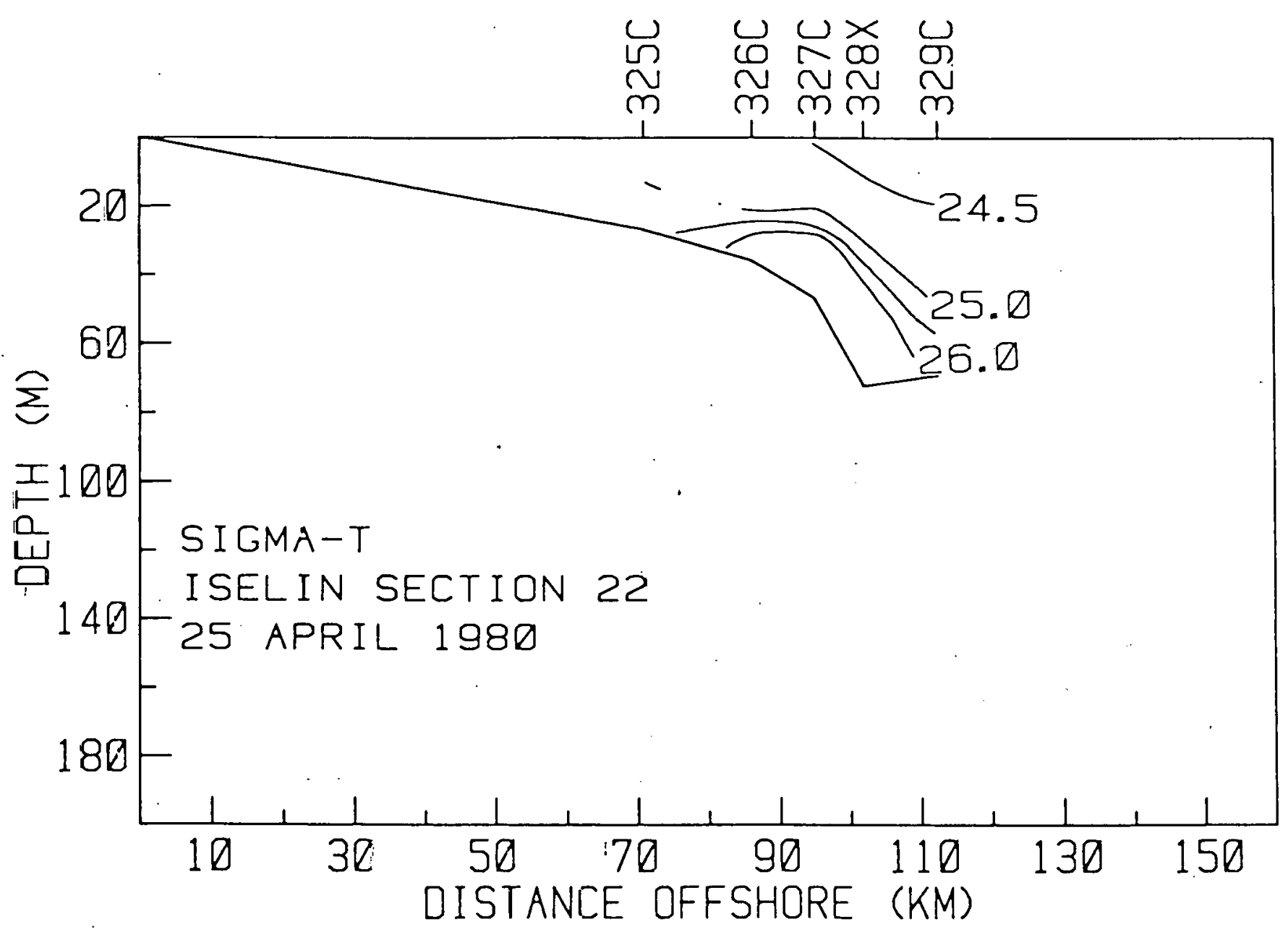




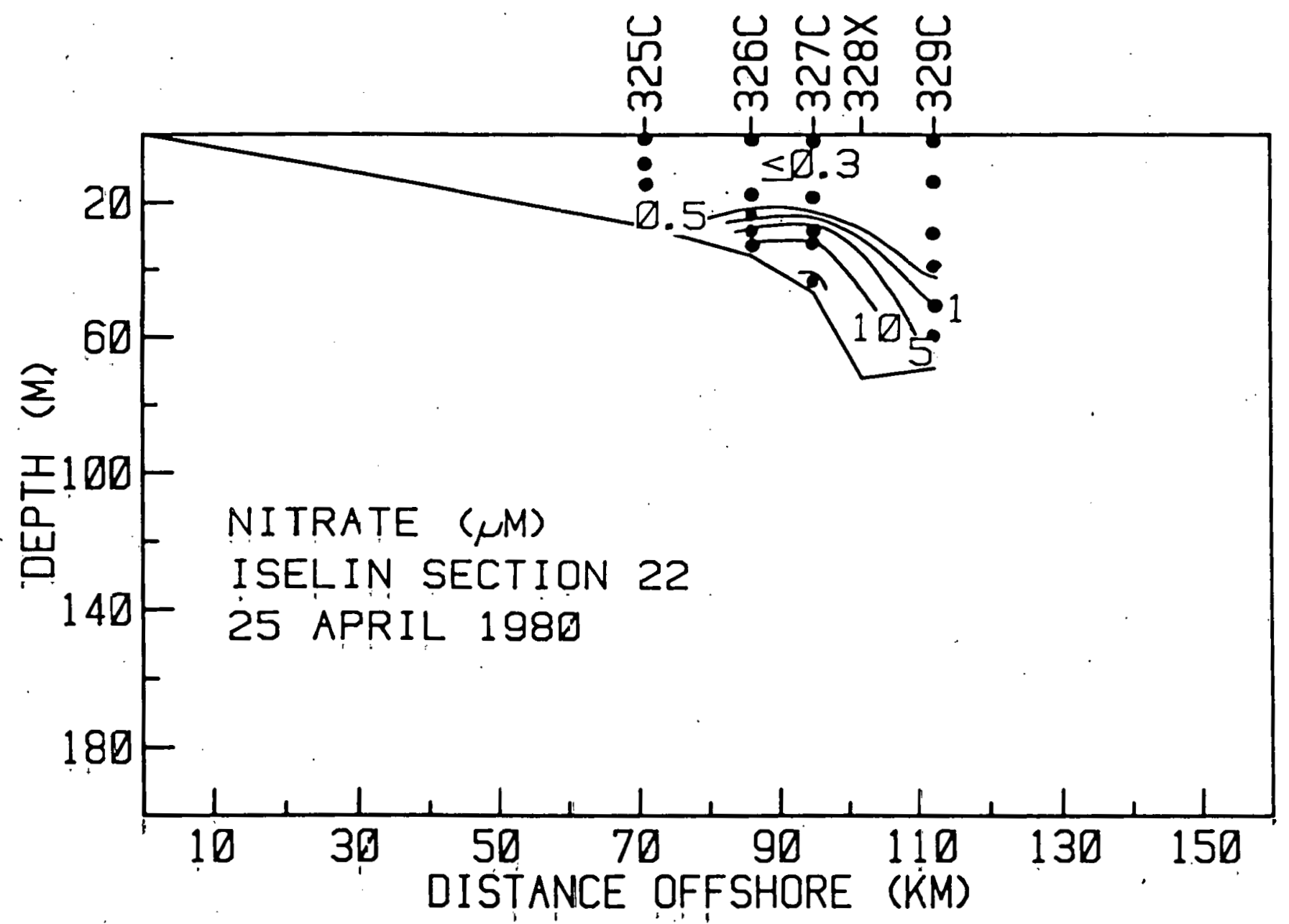




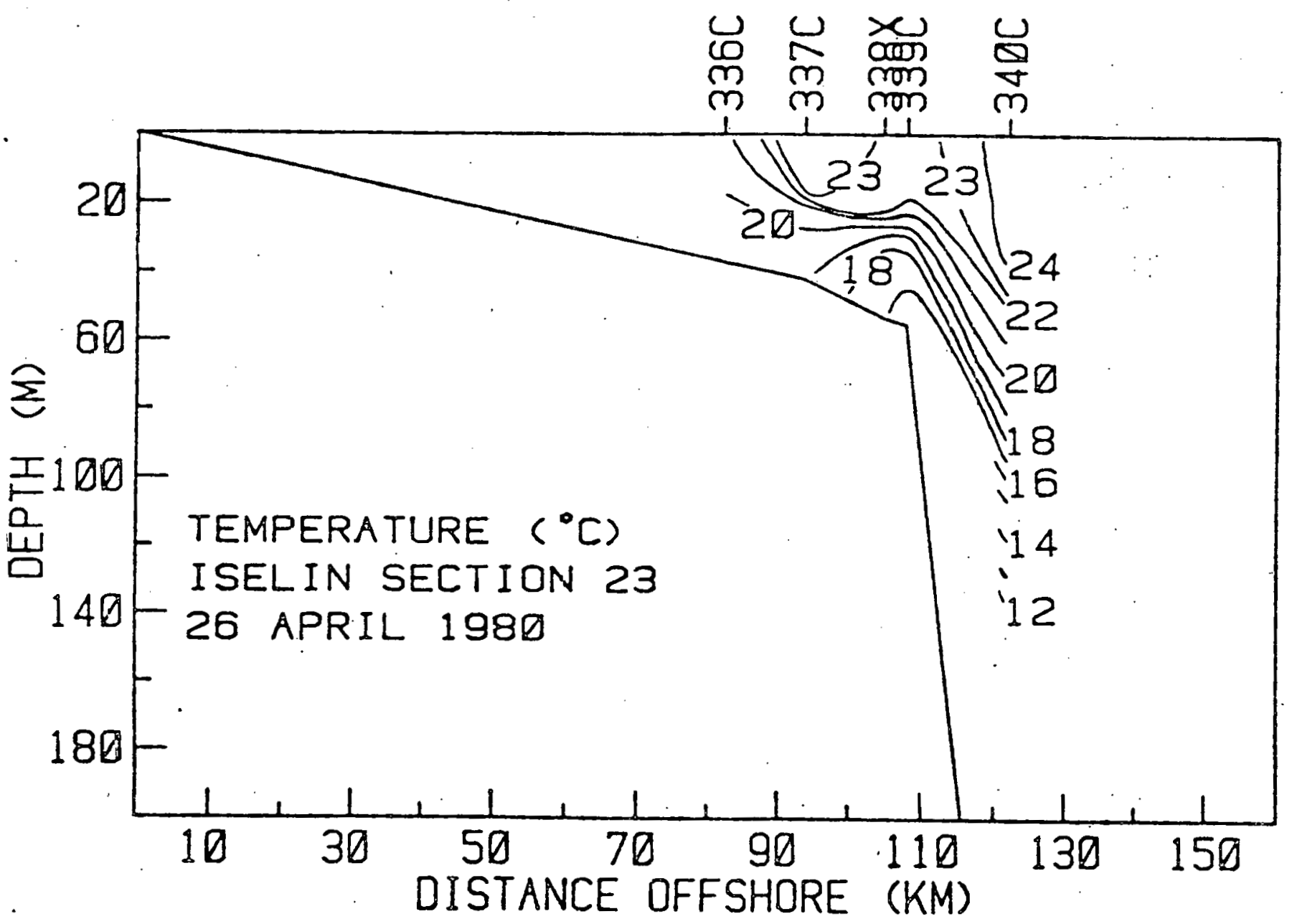




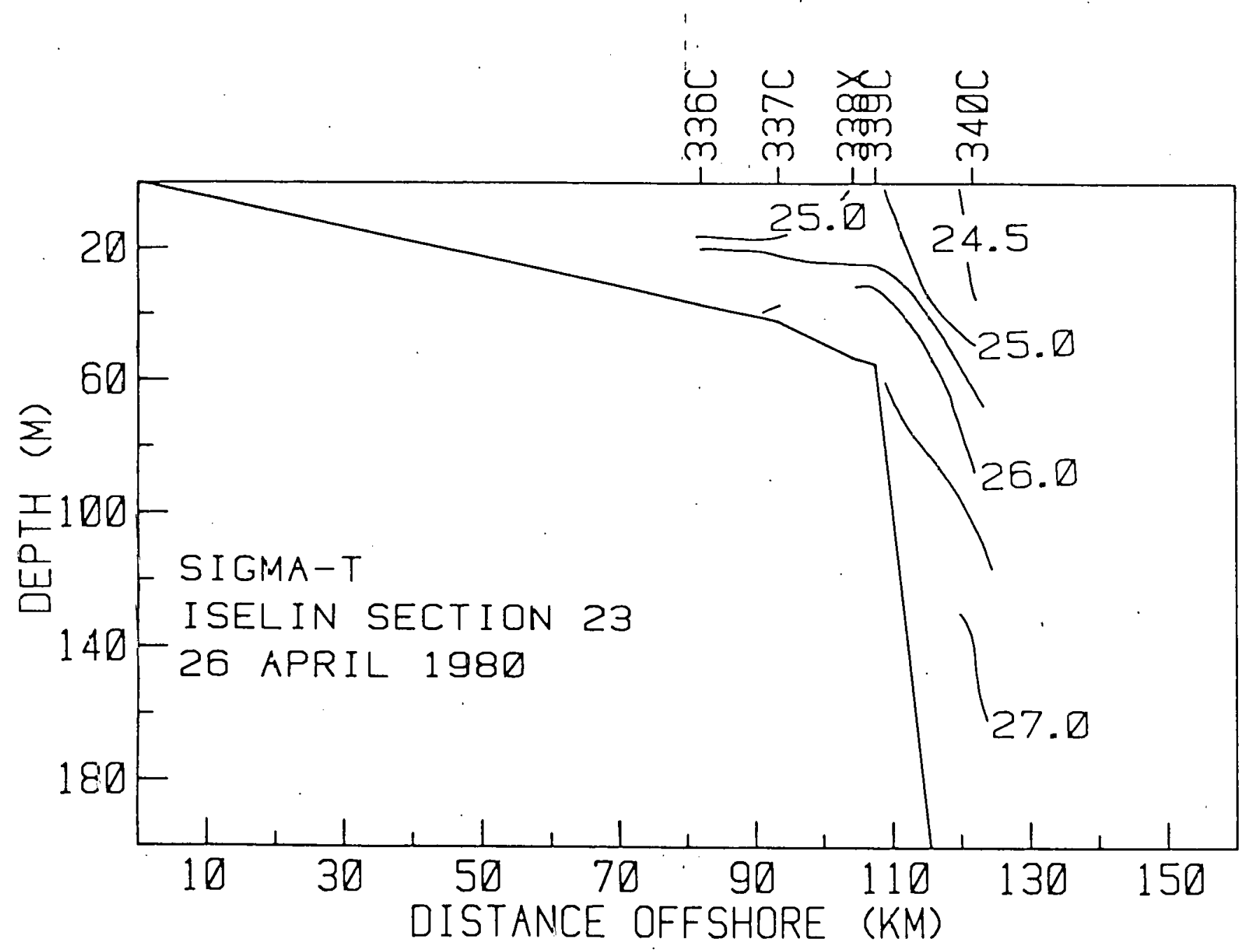



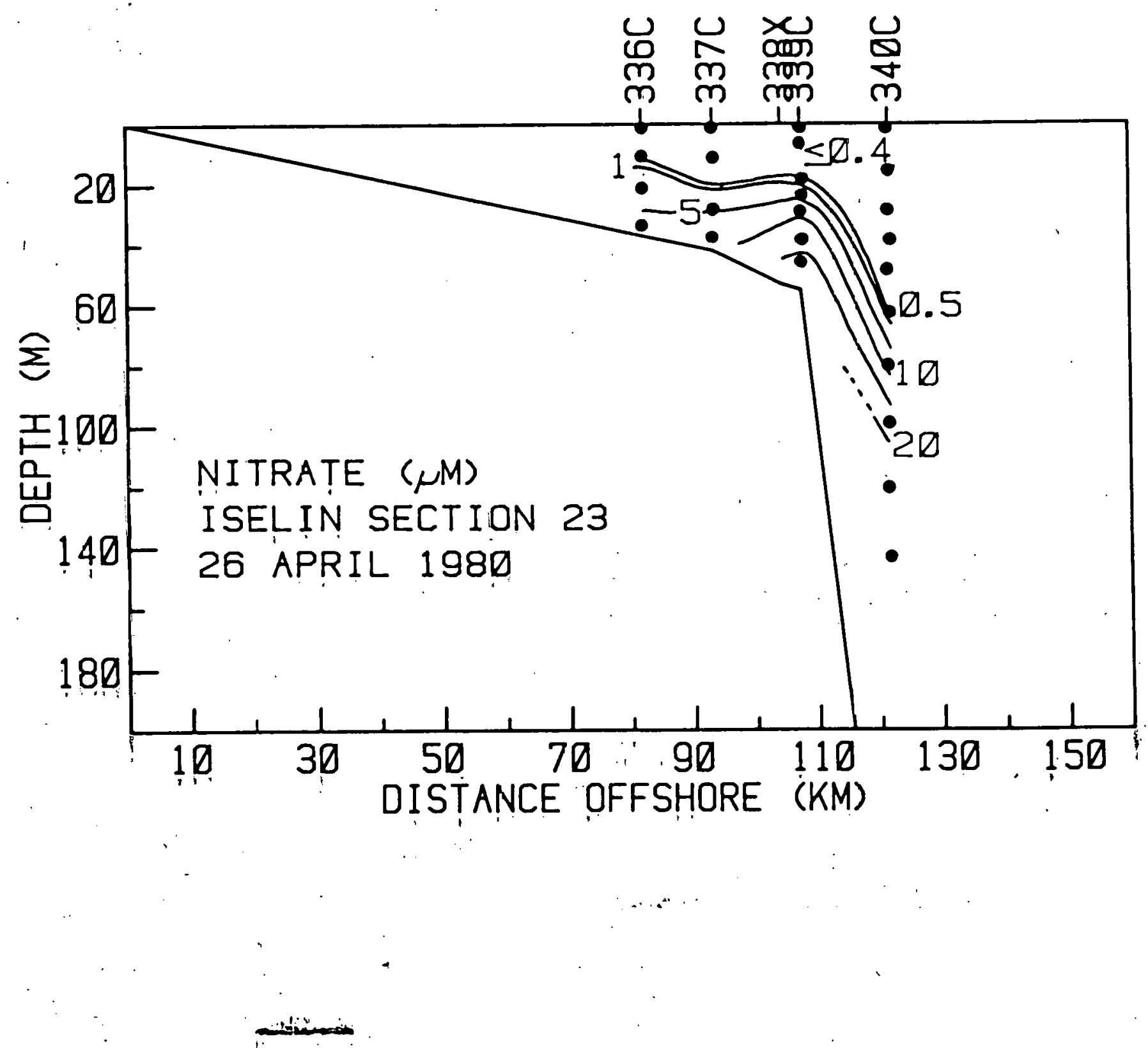


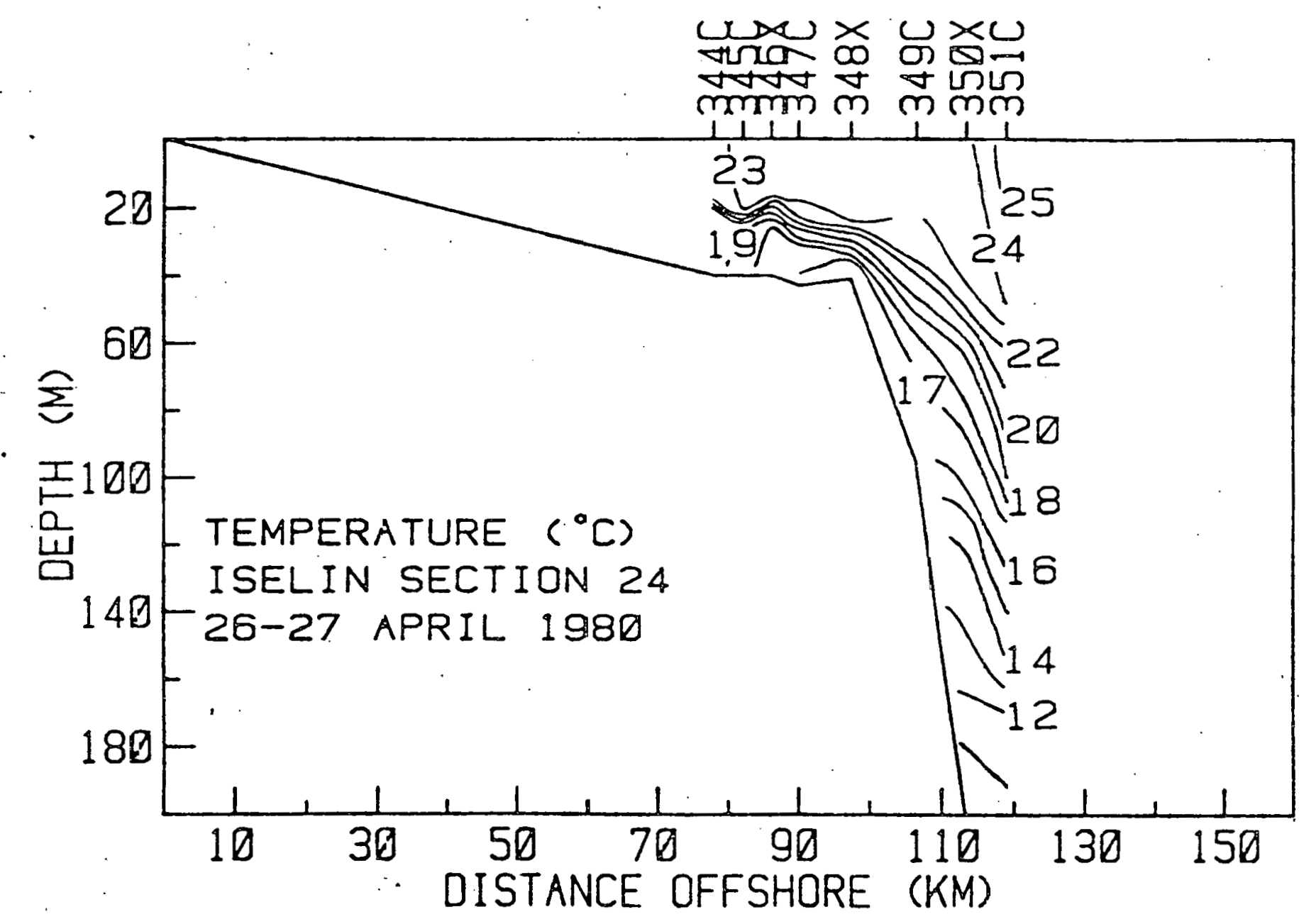




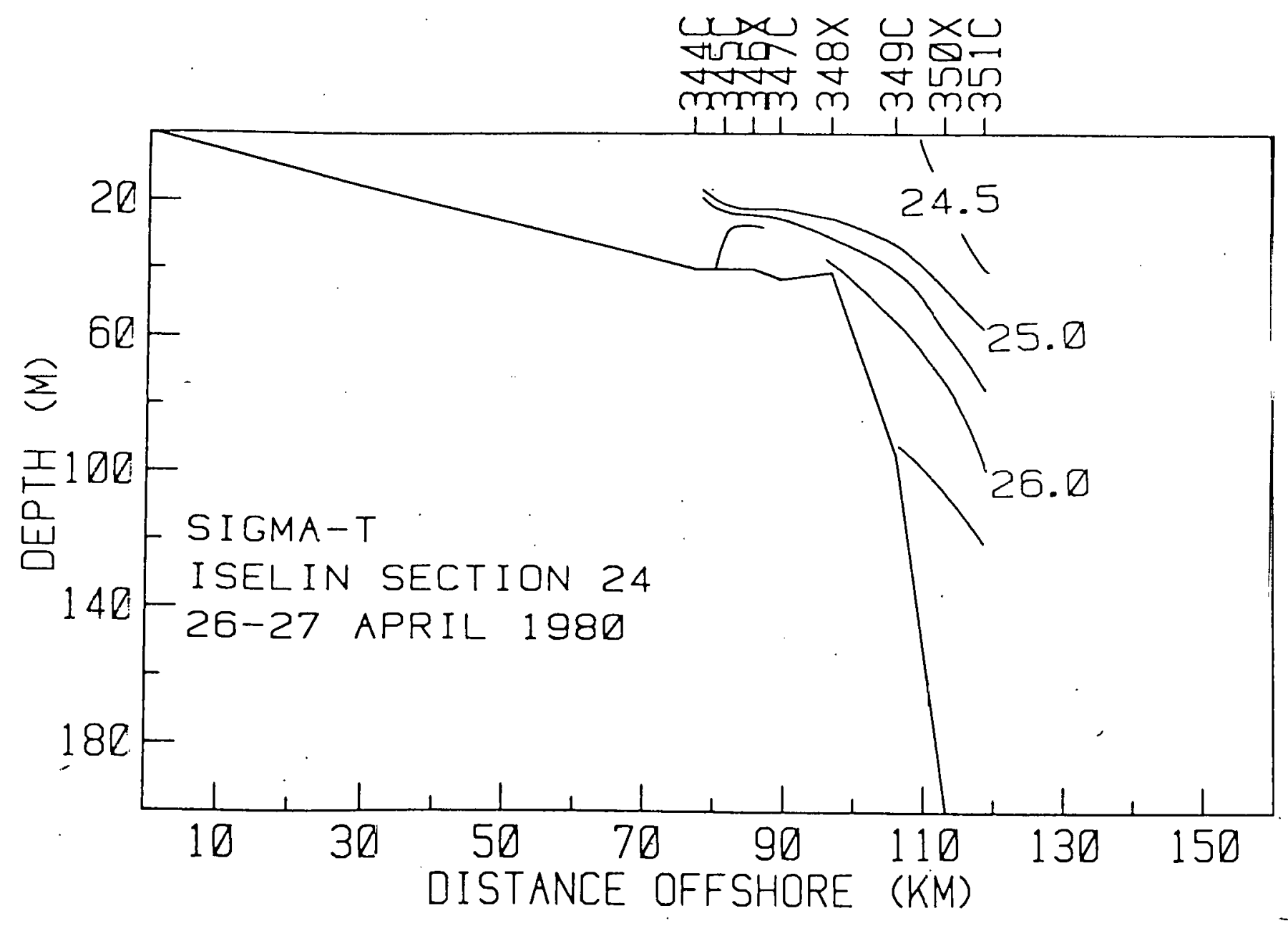




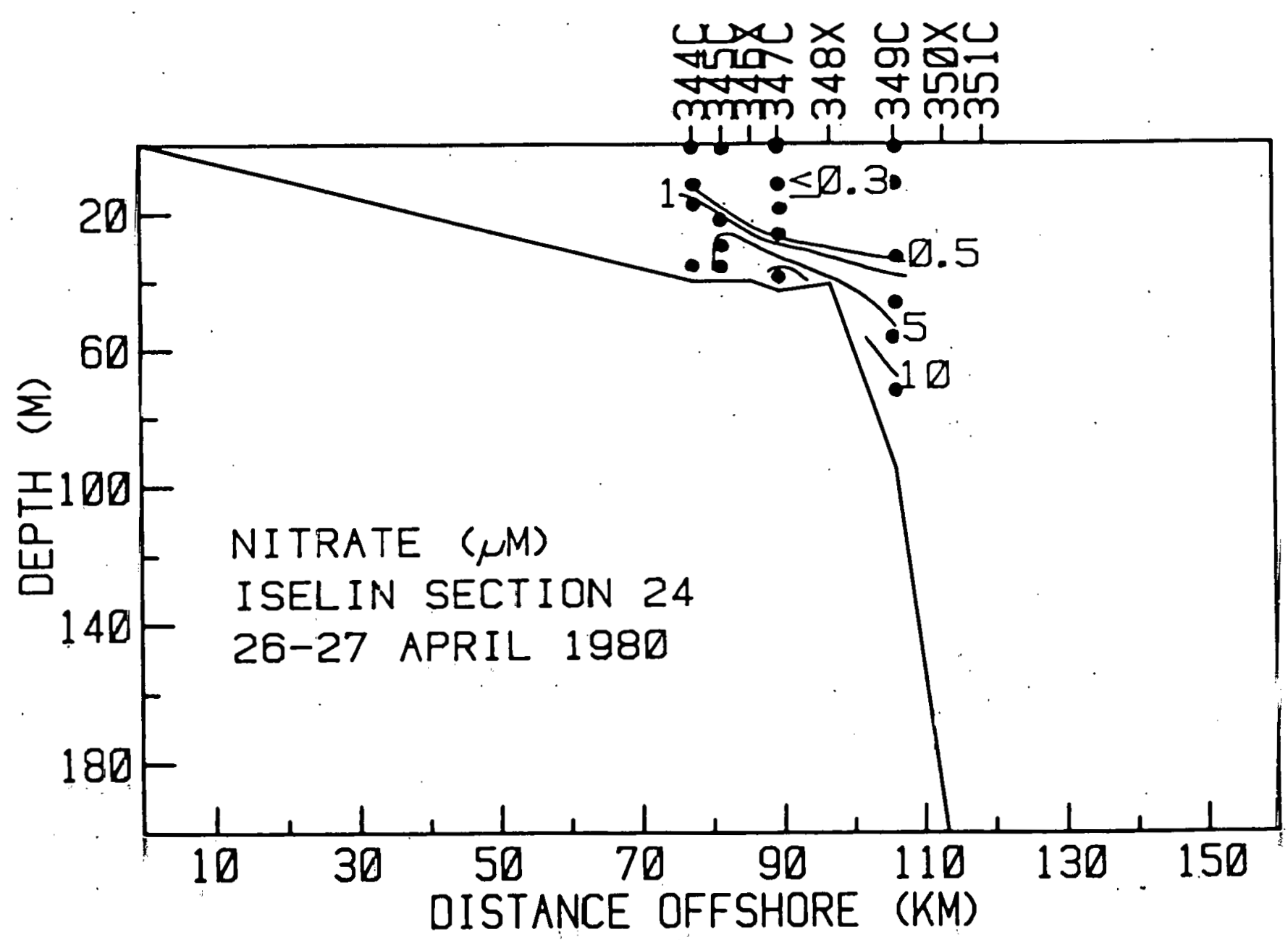

